\title{
Metazoan Parasites of Antarctic Fishes
}

\author{
Antarktik Balıkların Metazoon Parazitleri
}

\section{Mehmet Cemal Oğuz², Yahya Tepe, Mark C Belk1, Richard A Heckmann¹, Burçak Aslan², Meryem Gürgen², Rodney A Bray ${ }^{3}$, Ülker Akgül²}

'Department of Biology, Brigham Young University, Provo, Utah, United States

2Department of Biology, Atatürk University, Erzurum, Turkey

${ }^{3}$ Natural History Museum, Zoology, London, England

\section{ABSTRACT}

To date, there have been nearly 100 papers published on metazoan parasites of Antarctic fishes, but there has not yet been any compilation of a species list of fish parasites for this large geographic area. Herein, we provide a list of all documented occurrences of monogenean, cestode, digenean, acanthocephalan, nematode, and hirudinean parasites of Antarctic fishes. The list includes nearly 250 parasite species found in 142 species of host fishes. It is likely that there are more species of fish parasites, which are yet to be documented from Antarctic waters. (Turkiye Parazitol Derg 2015; 39: 174-8)

Keywords: Metazoan, parasites, Antarctic region, marine fishes

Received: 16.05 .2014

Accepted: 03.06.2015

\section{ÖZET}

Bugüne kadar Antarktik balıklarının metazoan parazitleri konusunda yayınlanmış olan yaklaşık 100'e yakın yayın basılmış olmasına karşın, bu geniş coğrafi alan içinde yer alan balıklara ait parazitlerle ilgili derlenmiş bir tür listesi bulunmamaktadır. Biz bu çalışmada da Antarktik balıklarının; Monogenea, Cestoda, Digenea, Acantosefal, Nematoda ve Hirudinea türlerinin bir listesini derledik. Bu liste de konak balıkların 142 türlerde bulunan yaklaşık 250 parazit türü tespit edilmiş olup, Antarktik sularda bulunmasına karşın hala listede de yer almayan birçok parazit bulunması da muhtemeldir. (Turkiye Parazitol Derg 2015; 39: 174-8)

Anahtar Sözcükler: Metazoon, parazitler, Antarktik Bölge, deniz balıkları

Geliş Tarihi: 16.05.2014

Kabul Tarihi: 03.06.2015

\section{INTRODUCTION}

Antarctic waters are inhabited by approximately 197 known species of fishes (www.fishbase.org). Description of the Antarctic fish fauna was largely accomplished by the middle of the last century; however, description of the metazoan parasites of Antarctic fishes and documentation of their occurrence in Antarctic waters lagged far behind. Most studies of fish parasites from Antarctic waters have been published within the last 40 years. To date (2010), there have been nearly 100 papers published on the metazoan parasites of Antarctic fishes. Of these, over half have been authored by Dr. Krzysztof Zdzitowiecki of the Polish Academy of Sciences in Warsaw, Poland, and his colleagues. This

Address for Correspondence / Yazışma Adresi: Dr. Mark C Belk, Department of Biology, Brigham Young University, Provo, Utah, United States. Phone: 801-422-4154 E-mail: mark_belk@byu.edu DOI: $10.5152 /$ tpd.2015.3661

OTelif hakkı 2015 Türkiye Parazitoloji Derneği - Makale metnine www.tparazitolderg.org web sayfasından ulaşılabilir. (0) Copyright 2015 Turkish Society for Parasitology - Available online at www.tparazitolderg.org 
compilation would only be a fraction of its current size without his dedicated efforts to document and publish the accounts of the parasites of Antarctic fishes over many decades.

Our objective is to provide a list of all documented occurrences of monogenean, cestode, digenean, acanthocephalan, nematode, and hirudinean parasites of Antarctic fishes. We have relied on reports published in the peer-reviewed literature. We believe that this list will be useful to a wide variety of researchers in taxonomy, ecology, and evolution.

\section{METHODS}

To compile this list of metazoan parasites of fishes of Antarctic waters, we attempted to locate all published accounts. In the list, we included every documented occurrence of a parasite species, specified as the host species (if given), gave the specific location or area, and cited the paper where the record was located. We used the original species name even if it differed from the current name, and we acknowledge that some of the names have been synonymized or changed. We arranged the species according to the traditional taxonomic group (i.e., monogenea, cestodes, digenea); then, we arranged them alphabetically according to family, genus within family, and species within genus.

\section{RESULTS AND CONCLUSION}

We located 97 papers (published between 1936 and 2010) reporting occurrences or descriptions of the metazoan parasites of fishes in Antarctic waters. Taxa reported included the following: 24 species, 10 genera, and 7 families of monogeneans; 73 species, 27 genera, and 12 families of cestodes; 87 species, 44 genera, and 10 families of digeneans; 20 species, 15 genera, and 3 families of nematodes; 27 species, 4 genera, and 3 families of acanthocephalans; and 18 species, 14 genera, and 1 family of hirudinean parasites (Table 1). These parasites were found in a total of approximately 142 species of host fishes. The fish species with the largest number of documented parasite species (>60 species of parasites) were Dissostichus eleginoides, Notothenia coriiceps, and Notothenia rossii. These fishes are abundant, and at least part of the number of parasite species documented for each fish species is likely to be a result of the large number of individuals examined.

This list may be incomplete because some species of Antarctic fishes have not been examined for parasites, and some fish species are represented by only a few occurrences. There are 197 fish species described from Antarctic waters, and metazoan parasites have been documented from approximately 142 of those species. Examination of other specimens of these same species and other fish species may yield more metazoan parasite species. Some of the parasite groups included here, such as monogeneans, are among the most host-specific parasites; therefore, examination of new potential host species may lead to the identification of new monogenean species. However, more studies still remains to be conducted. 
Table 1. List of all published occurrences of metazoan parasites of fishes in Antarctic waters.

\begin{tabular}{|c|c|c|c|c|c|c|}
\hline Index & Phylum & Family & Species & Host & Location & References \\
\hline 1 & \multirow{24}{*}{ MONOGENEA } & Acanthocotylidae & Acanthocotyle sp. & blank & blank & Monticelli (1888) \\
\hline 2 & & & Acanthocotyle sp. & HOST NAME NOT MENTIONED & Kerguelen Subregion & Lyadov (1985) \\
\hline 3 & & Capsalidae & blank & blank & blank & Kovaljova \& Gaevskaya (1977) \\
\hline 4 & & & Pseudobenedenia antarcticus & Notothenia kempi & Antarctic Region & Kovaljova \& Gaevskaya (1977) \\
\hline 5 & & & Pseudobenedenia antarcticus & HOST NAME NOT MENTIONED & Glacial Subregion & Lyadov (1985) \\
\hline 6 & & & Pseudobenedenia branchiale & blank & blank & $\begin{array}{c}\text { Timofeeva, Gaevskaya \& } \\
\text { Kovalyova (1987) }\end{array}$ \\
\hline 7 & & & Pseudobenedenia branchiale & Dissostichus eleginoides & South Georgia Island & Gaevskaya et al. (1990) \\
\hline 8 & & & Pseudobenedenia dissostichi & blank & blank & $\begin{array}{c}\text { Timofeeva, Gaevskaya \& } \\
\text { Kovalyova (1987) }\end{array}$ \\
\hline 9 & & & Pseudobenedenia dissostichi & Dissostichus eleginoides & Falkland Patagonia & Gaevskaya et al. (1990) \\
\hline 10 & & & Pseudobenedenia dissostichi & Dissostichus eleginoides & South Georgia Island & Gaevskaya et al. (1990) \\
\hline 11 & & & Pseudobenedenia dissostichi & Dissostichus eleginoides & Falkland Islands & Brickle et al. (2006) \\
\hline 12 & & & Pseudobenedenia dissostichi & Dissostichus eleginoides & Heard Island & Rohde et al. (1998) \\
\hline 13 & & & Pseudobenedenia dissostichi & Dissostichus eleginoides & Shag Rocks & Brickle et al. (2005) \\
\hline 14 & & & Pseudobenedenia lauriei & blank & blank & Szidat (1965) \\
\hline 15 & & & Pseudobenedenia lauriei & Notothenia neglecta & $\begin{array}{c}\text { Bhaia Scotia La Isla } \\
\text { Laurie (Orcadas del sur, } \\
\text { Argentina) }\end{array}$ & Szidat (1965) \\
\hline 16 & & & Pseudobenedenia lauriei & Notothenia neglecta & Isle Laurie & Szidat (1966) \\
\hline 17 & & & Pseudobenedenia lauriei & Notothenia neglecta & Orcadas Del Sur & Szidat (1967) \\
\hline 18 & & & Pseudobenedenia nototheniae & blank & blank & Johnston (1931) \\
\hline 19 & & & Pseudobenedenia nototheniae & Dissostichus eleginoides & Kerguelen Subregion & Parukhin \& Lyadov (1981) \\
\hline 20 & & & Pseudobenedenia nototheniae & Dissostichus eleginoides & Crozet Islands & Parukhin \& Lyadov (1981) \\
\hline 21 & & & Pseudobenedenia nototheniae & Dissostichus eleginoides & Ob Bank & Parukhin \& Lyadov (1981) \\
\hline 22 & & & Pseudobenedenia nototheniae & Dissostichus eleginoides & Lena Bank & Parukhin \& Lyadov (1981) \\
\hline 23 & & & Pseudobenedenia nototheniae & Dissostichus eleginoides & Kerguelen Subregion & Parukhin \& Lyadov (1982) \\
\hline 24 & & & Pseudobenedenia nototheniae & Dissostichus eleginoides & Skif Bank & Parukhin \& Lyadov (1982) \\
\hline
\end{tabular}




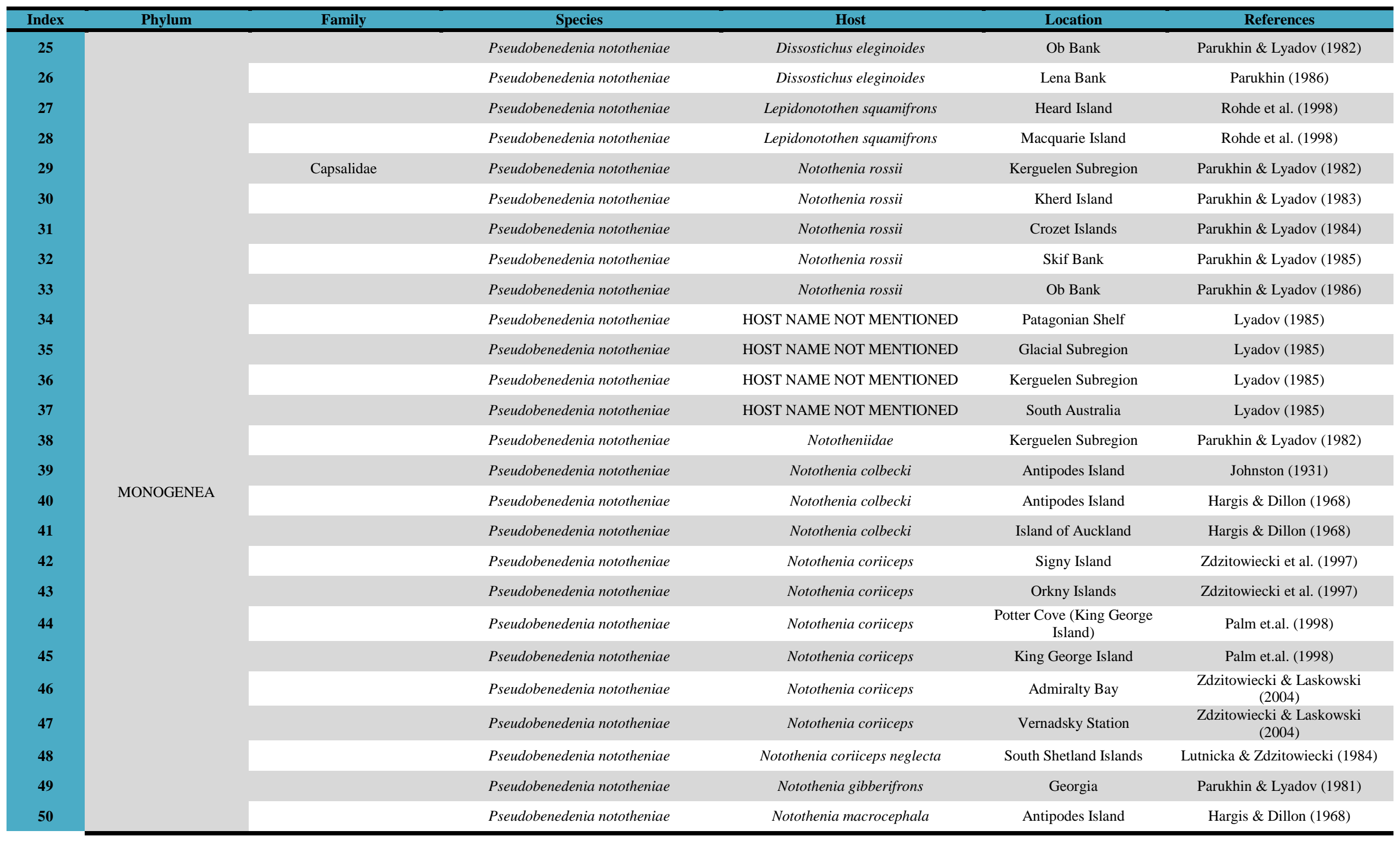




\begin{tabular}{|c|c|c|c|c|c|c|}
\hline Index & Phylum & Family & Species & Host & Location & References \\
\hline 51 & & & Pseudobenedenia nototheniae & Notothenia macrocephala & Macquarie Island & Hargis \& Dillon (1968) \\
\hline 52 & & & Pseudobenedenia nototheniae & Notothenia ramsayi & Folkland & Parukhin \& Lyadov (1981) \\
\hline 53 & & & Pseudobenedenia nototheniae & Notothenia squamifrons & Lena Bank & Parukhin (1986) \\
\hline 54 & & & Pseudobenedenia nototheniae & Notothenia squamifrons & Ob Bank & Parukhin (1986) \\
\hline 55 & & & Pseudobenedenia nototheniae & Notothenia rossii & $\begin{array}{l}\text { Kerguelen Subregion } \\
\text { Islands }\end{array}$ & Hargis \& Dillon (1968) \\
\hline 56 & & & Pseudobenedenia nototheniae & Notothenia rossii & Kerguelen Subregion & Parukhin \& Lyadov (1981) \\
\hline 57 & & & Pseudobenedenia nototheniae & Notothenia rossii & Kherd Island & Parukhin \& Lyadov (1981) \\
\hline 58 & \multirow{20}{*}{ MONOGENEA } & Capsalidae & Pseudobenedenia nototheniae & Notothenia rossii & Crozet Islands & Parukhin \& Lyadov (1981) \\
\hline 59 & & & Pseudobenedenia nototheniae & Notothenia rossii & Skif Bank & Parukhin \& Lyadov (1981) \\
\hline 60 & & & Pseudobenedenia nototheniae & Notothenia rossii & Ob Bank & Parukhin \& Lyadov (1981) \\
\hline 61 & & & Pseudobenedenia nototheniae & Notothenia rossii & Borge Bay & Hoogesteger \& White (1981) \\
\hline 62 & & & Pseudobenedenia nototheniae & Notothenia rossii & Signy Island & Hoogesteger \& White (1981) \\
\hline 63 & & & Pseudobenedenia nototheniae & Notothenia rossii & Lena Bank & Parukhin (1986) \\
\hline 64 & & & Pseudobenedenia nototheniae & Notothenia rossii & Ob Bank & Parukhin (1986) \\
\hline 65 & & & Pseudobenedenia nototheniae & Notothenia rossii marmorata & South Shetland Islands & Lutnicka \& Zdzitowiecki (1984) \\
\hline 66 & & & Pseudobenedenia nototheniae & Trematomus bernacchii & Mcmurdo Sound & Hargis \& Dillon (1968) \\
\hline 67 & & & Pseudobenedenia shorti & blank & blank & Hargis \& Dillon (1968) \\
\hline 68 & & & Pseudobenedenia shorti & Rhigophila dearboni & Mcmurdo Sound & Hargis \& Dillon (1968) \\
\hline 69 & & & Pseudobenedenia shorti & Trematomus bernacchii, & Mcmurdo Sound & Hargis \& Dillon (1968) \\
\hline 70 & & & Pseudobenedenia shorti & Trematomus centronotus & Mcmurdo Sound & Hargis \& Dillon (1968) \\
\hline 71 & & & Pseudobenedenia shorti & Trematomus hansoni & Mcmurdo Sound & Hargis \& Dillon (1968) \\
\hline 72 & & & Pseudobenedenia shorti & Trematomus bernacchii, & Windmill Islands & Hargis \& Dillon (1968) \\
\hline 73 & & & Pseudobenedenia shorti & Trematomus centronotus & Windmill Islands & Hargis \& Dillon (1968) \\
\hline 74 & & & Pseudobenedenia shorti & Trematomus hansoni & Windmill Islands & Hargis \& Dillon (1968) \\
\hline 75 & & & Pseudobenedenia shorti & Trematomus sp. & Ramp Cove & Hargis \& Dillon (1968) \\
\hline 76 & & & Pseudobenedenia sp. & blank & blank & Johnston (1931) \\
\hline 77 & & & Pseudobenedenia sp. & Trematomus borchgrevinski & Mcmurdo Sound & Hargis \& Dillon (1968) \\
\hline
\end{tabular}




\begin{tabular}{|c|c|c|c|c|c|c|}
\hline Index & Phylum & Family & Species & Host & Location & References \\
\hline 78 & & & Pseudobenedenoides branchicola & blank & blank & Szidat (1969) \\
\hline 79 & & & Pseudobenedenoides branchicola & Trematomus bernacchii & Auckland & Szidat (1969) \\
\hline 80 & & & Pseudobenedenoides branchicola & Trematomus bernacchii & Mcmurdo Sound & Moser \& Cowen (1991) \\
\hline 81 & & & Pseudobenedenoides shorti & blank & blank & Hargis \& Dillon (1968) \\
\hline 82 & & & Pseudobenedenoides shorti & Trematomus bernacchii & Antarctica & Rohde et al. (1995) \\
\hline 83 & & & Pseudobenedenoides shorti & Trematomus bernacchii, & Casey Station & Rohde et al. (1998) \\
\hline 84 & & & Pseudobenedenoides shorti & Trematomus hansoni & Antarctica & Rohde et al. (1995) \\
\hline 85 & & & Pseudobenedenoides shorti & Trematomus hansoni & Casey Station & Rohde et al. (1998) \\
\hline 86 & & & Pseudobenedenoides shorti & HOST NAME NOT MENTIONED & Glacial Subregion & Lyadov (1985) \\
\hline 87 & \multirow{18}{*}{ MONOGENEA } & Diclidophoridae & Diclidophoridae sp. & blank & blank & Cerfontaine (1895) \\
\hline 88 & & & Diclidophoridae sp. & Macrourus holotrachys & Heard Island & Rohde et al. (1998) \\
\hline 89 & & & Diclidophora antarctica & Macrourus holotrachys & Antarctica & Rohde et al. (1995) \\
\hline 90 & & & Macruricotyle claviceps & blank & blank & Mamaev \& Lyadov (1975) \\
\hline 91 & & & Macruricotyle claviceps & Macrourus carinatus & $\begin{array}{c}\text { Falkland (Malvinas) } \\
\text { Islands }\end{array}$ & Gaevskaya \& Rodyuk (1988) \\
\hline 92 & & & Macruricotyle claviceps & HOST NAME NOT MENTIONED & Kerguelen Subregion & Lyadov (1985) \\
\hline 93 & & Gyrodactylidae & Gyrodactylidae spp. & blank & blank & Cobbold (1864) \\
\hline 94 & & & Gyrodactylidae spp. & Trematomus eulepidotus & Antarctica & Rohde et al. (1995) \\
\hline 95 & & & Gyrodactylidae spp. & Lepidonotothen squamifrons & Antarctica & Rohde et al. (1995) \\
\hline 96 & & & Gyrodactylus sp. & blank & blank & Von Nordmann (1832) \\
\hline 97 & & & Gyrodactylus sp. & Lepidonotothen kempi & Prydz Bay & Rohde et al. (1998) \\
\hline 98 & & & Gyrodactylus sp. & Lepidonotothen mizops & Heard Island & Rohde et al. (1998) \\
\hline 99 & & & Gyrodactylus sp. & Lepidonotothen mizops & Antarctic Region & Rohde et al. (1998) \\
\hline 100 & & & Gyrodactylus sp. & Lepidonotothen nudifrons & Admiralty Bay & Rokicka (2009) \\
\hline 101 & & & Gyrodactylus sp. & Lepidonotothen nudifrons & South Shetland Islands & Rokicka (2009) \\
\hline 102 & & & Gyrodactylus sp. & Lepidonotothen squamifrons & Heard Island & Rohde et al. (1998) \\
\hline 103 & & & Gyrodactylus sp. & Lepidonotothen squamifrons & Macquarie Island & Rohde et al. (1998) \\
\hline 104 & & & Gyrodactylus sp. & Notothenia coriiceps & Admiralty Bay & Rokicka (2009) \\
\hline
\end{tabular}




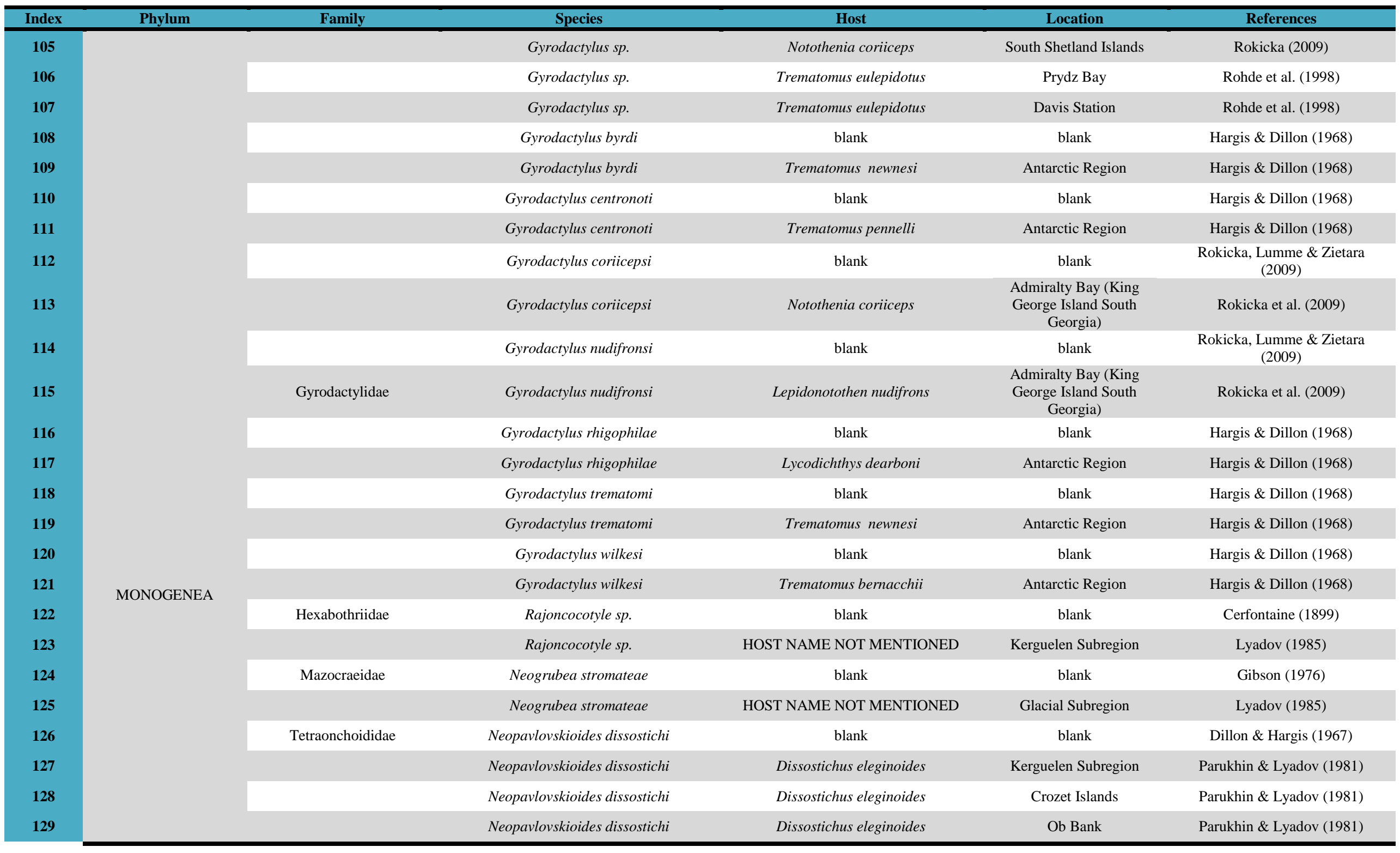




\begin{tabular}{|c|c|c|c|c|c|c|}
\hline Index & Phylum & Family & Species & Host & Location & References \\
\hline 130 & & & Neopavlovskioides dissostichi & Dissostichus eleginoides & Lena Bank & Parukhin \& Lyadov (1981) \\
\hline 131 & & & Neopavlovskioides dissostichi & Dissostichus eleginoides & Shag Rocks & Parukhin \& Lyadov (1981) \\
\hline 132 & & & Neopavlovskioides dissostichi & Dissostichus eleginoides & Kerguelen Subregion & Parukhin \& Lyadov (1982) \\
\hline 133 & & & Neopavlovskioides dissostichi & Dissostichus eleginoides & Kherd Island & Parukhin \& Lyadov (1982) \\
\hline 134 & & & Neopavlovskioides dissostichi & Dissostichus eleginoides & Ob Bank & Parukhin \& Lyadov (1982) \\
\hline 135 & & & Neopavlovskioides dissostichi & Dissostichus eleginoides & Lena Bank & Parukhin \& Lyadov (1982) \\
\hline 136 & & & Neopavlovskioides dissostichi & Dissostichus eleginoides & Ob Bank & Parukhin (1986) \\
\hline 137 & & & Neopavlovskioides dissostichi & Dissostichus eleginoides & Lena Bank & Parukhin (1986) \\
\hline 138 & & & Neopavlovskioides dissostichi & Nototheniidae & Kerguelen Subregion & Parukhin \& Lyadov (1982) \\
\hline 139 & & & Neopavlovskioides dissostichi & HOST NAME NOT MENTIONED & Kerguelen Subregion & Lyadov (1985) \\
\hline 140 & & & Neopavlovskioides dissostichi & blank & blank & Dillon \& Hargis (1968) \\
\hline 141 & & & Neopavlovskioides dissostichi & Dissostichus eleginoides & Heard Island & Rohde et al. (1998) \\
\hline 142 & & & Neopavlovskioides georgianus & blank & blank & Kovaljova \& Gaevskaya (1977) \\
\hline 143 & & & Neopavlovskioides georgianus & Dissostichus eleginoides & Antarctic Region & Kovaljova \& Gaevskaya (1977) \\
\hline 144 & \multirow{13}{*}{ MONOGENEA } & Tetraonchoididae & Neopavlovskioides georgianus & Dissostichus eleginoides & Falkland Patagonia & Gaevskaya et al. (1990) \\
\hline 145 & & & Neopavlovskioides georgianus & Dissostichus eleginoides & South Georgia Island & Gaevskaya et al. (1990) \\
\hline 146 & & & Neopavlovskioides georgianus & Dissostichus eleginoides & Shag Rocks & Brickle et al. (2005) \\
\hline 147 & & & Neopavlovskioides georgianus & Dissostichus eleginoides & South Georgia Island & Brickle et al. (2005) \\
\hline 148 & & & Neopavlovskioides georgianus & Dissostichus eleginoides & Prince Edward Island & Brickle et al. (2005) \\
\hline 149 & & & Neopavlovskioides georgianus & Dissostichus eleginoides & Heard Island & Brickle et al. (2005) \\
\hline 150 & & & Neopavlovskioides georgianus & Dissostichus eleginoides & Macquarie Island & Brickle et al. (2005) \\
\hline 151 & & & Neopavlovskioides georgianus & Dissostichus eleginoides & Ross Sea & Brickle et al. (2005) \\
\hline 152 & & & Neopavlovskioides georgianus & Dissostichus eleginoides & Falkland Islands & Brickle et al. (2006) \\
\hline 153 & & & Neopavlovskioides georgianus & HOST NAME NOT MENTIONED & Glacial Subregion & Lyadov (1985) \\
\hline 154 & & & Pavlovskioides antarcticus & blank & blank & $\begin{array}{c}\text { Bychowsky, Gussev \& Nagibina } \\
\text { (1965) }\end{array}$ \\
\hline 155 & & & Pavlovskioides antarcticus & Trematomus bernacchii & Antarctica & Rohde et al. (1995) \\
\hline 156 & & & Pavlovskioides antarcticus & Trematomus bernacchii & Casey Station & Rohde et al. (1998) \\
\hline
\end{tabular}




\begin{tabular}{|c|c|c|c|c|c|c|}
\hline Index & Phylum & Family & Species & Host & Location & References \\
\hline 157 & & & Pavlovskoides prudhoei & blank & blank & Gibson (1976) \\
\hline 158 & & & Pavlovskoides prudhoei & HOST NAME NOT MENTIONED & Glacial Subregion & Lyadov (1985) \\
\hline 159 & & & Pavlovskioides trematomi & blank & blank & Dillon \& Hargis (1968) \\
\hline 160 & & & Pavlovskioides trematomi & Trematomus loennbergi & Prydz Bay & Rohde et al. (1998) \\
\hline 161 & \multirow{10}{*}{ DIGENEA } & blank & Digenean sp. & Dissostichus eleginoides & Heard Island & Brickle et al. (2005) \\
\hline 162 & & Azygiidae & Otodistomum sp. & blank & blank & Stafford (1904) \\
\hline 163 & & & Otodistomum sp. & HOST NAME NOT MENTIONED & Glacial Subregion & Lyadov (1985) \\
\hline 164 & & & Otodistomum cestoides (metacercaria) & blank & blank & van Beneden (1871) \\
\hline 165 & & & Otodistomum cestoides (metacercaria) & Artedidraconidae & Weddell Sea & Zdzitowiecki (2002c) \\
\hline 166 & & & Otodistomum cestoides (metacercaria) & Bathydraconidae & Weddell Sea & Zdzitowiecki (2002d) \\
\hline 167 & & & Otodistomum cestoides & blank & blank & van Beneden (1871) \\
\hline 168 & & & Otodistomum cestoides & Artedidraco skottsbergi & Weddell Sea & Zdzitowiecki (2002c) \\
\hline 169 & & & Otodistomum cestoides & Batyraja eatoni & South Shetland Islands & Zdzitowiecki (1991) \\
\hline 170 & & & Otodistomum cestoides & Racovitzia glacialis & Weddell Sea & Zdzitowiecki (2002d) \\
\hline 171 & \multirow{13}{*}{ DIGENEA } & & Otodistomum cestoides & HOST NAME NOT MENTIONED & Glacial Subregion & Lyadov (1985) \\
\hline 172 & & Derogenidae & Derogenes johnstoni & blank & blank & Prudhoe \& Bray (1973) \\
\hline 173 & & & Derogenes johnstoni & Artedidraco loennbergi & Weddell Sea & Zdzitowiecki (2002c) \\
\hline 174 & & & Derogenes johnstoni & Artedidraco orianae & Weddell Sea & Zdzitowiecki (2002c) \\
\hline 175 & & & Derogenes johnstoni & Artedidraco shackletoni & $65^{\circ} 48^{\prime} \mathrm{S} 53^{\circ} 16^{\prime} \mathrm{E}$ & Prudhoe \& Bray (1973) \\
\hline 176 & & & Derogenes johnstoni & Artedidraco shackletoni & $66^{\circ} 45^{\prime} \mathrm{S} 62^{\circ} 03^{\prime} \mathrm{E}$ & Prudhoe \& Bray (1973) \\
\hline 177 & & & Derogenes johnstoni & Chionodraco hamatus & Adelie Land & Zdzitowiecki et.al. (1998) \\
\hline 178 & & & Derogenes johnstoni & Chionodraco hamatus & Weddell Sea & Zdzitowiecki (2002b) \\
\hline 179 & & & Derogenes johnstoni & Chionodraco hamatus & $\begin{array}{c}\text { Ross Sea (Terra Nova } \\
\text { Bay) }\end{array}$ & Zdzitowiecki et al. (1993b) \\
\hline 180 & & & Derogenes johnstoni & Cryodraco antarcticus & $\begin{array}{c}\text { Ross Sea (Terra Nova } \\
\text { Bay) }\end{array}$ & Zdzitowiecki et al. (1993b) \\
\hline 181 & & & Derogenes johnstoni & Dolloidraco longedorsalis & Weddell Sea & Zdzitowiecki (2002c) \\
\hline 182 & & & Derogenes johnstoni & Notothenia coriiceps & Adelie Land & Zdzitowiecki et.al. (1998) \\
\hline 183 & & & Derogenes johnstoni & Trematomus hansoni & Adelie Land & Zdzitowiecki et.al. (1998) \\
\hline
\end{tabular}




\begin{tabular}{|c|c|c|c|c|c|c|}
\hline Index & Phylum & Family & Species & Host & Location & References \\
\hline 184 & & & Derogenes johnstoni & Trematomus loennbergi & $\begin{array}{c}\text { Ross Sea (Terra Nova } \\
\text { Bay) }\end{array}$ & Zdzitowiecki et al. (1993b) \\
\hline 185 & & & Derogenes johnstoni & Trematomus loennbergi & Weddell Sea & Zdzitowiecki (2002b) \\
\hline 186 & & & Derogenes johnstoni & $\begin{array}{c}\text { Trematomus pennellii (see Gon and } \\
\text { Heemstra 1990) }\end{array}$ & $\begin{array}{c}\text { Ross Sea (Terra Nova } \\
\text { Bay) }\end{array}$ & Zdzitowiecki et al. (1993b) \\
\hline 187 & & & Derogenes johnstoni & Pagothenia bernacchii & $\begin{array}{c}\text { Ross Sea (Terra Nova } \\
\text { Bay) }\end{array}$ & Zdzitowiecki et al. (1993b) \\
\hline 188 & & & Derogenes johnstoni & Paraliparis trilobodon & Weddell Sea & Zdzitowiecki (2003) \\
\hline 189 & & & Derogenes varicus & Paraliparis trilobodon & Weddell Sea & Müller (1784) \\
\hline 190 & & & Derogenes varicus & Paraliparis trilobodon & Weddell Sea & Looss (1901) \\
\hline 191 & & & Derogenes varicus & Chaenichthys velifer & Kerguelen Subregion & Parukhin \& Lyadov (1981) \\
\hline 192 & & & Derogenes varicus & Chaenichthys velifer & Kerguelen Subregion & Lyadov et al. (1981) \\
\hline 193 & & & Derogenes varicus & Champsocephalus gunnari & $49^{\circ} 28^{\prime} \mathrm{S} 70^{\circ} 33^{\prime} \mathrm{E}$ & Prudhoe \& Bray (1973) \\
\hline 194 & & & Derogenes varicus & Champsocephalus gunnari & $\begin{array}{l}\text { Off Entrance To Royal } \\
\text { Sound (Kerguelen } \\
\text { Subregion) }\end{array}$ & Prudhoe \& Bray (1973) \\
\hline 195 & & & Derogenes varicus & Dissostichus eleginoides & Kerguelen Subregion & Parukhin \& Lyadov (1981) \\
\hline 196 & & & Derogenes varicus & Dissostichus eleginoides & Kerguelen Subregion & Parukhin \& Lyadov (1982) \\
\hline 197 & & & Derogenes varicus & Dissostichus eleginoides & Falkland-Patagonian Shelf & Gaevskaya et al. (1990) \\
\hline 198 & & & Derogenes varicus & Dissostichus eleginoides & Prince Edward Island & Brickle et al. (2005) \\
\hline 199 & & & Derogenes varicus & Dissostichus eleginoides & Heard Island & Brickle et al. (2005) \\
\hline 200 & & & Derogenes varicus & Dissostichus eleginoides & Macquarie Island & Brickle et al. (2005) \\
\hline 201 & & Derogenidae & Derogenes varicus & Dissostichus eleginoides & Falkland Islands & Brickle et al. (2006) \\
\hline 202 & & & Derogenes varicus & Harpagifer bispinis & A.A.E. & Prudhoe \& Bray (1973) \\
\hline 203 & & & Derogenes varicus & Harpagifer bispinis & Macquarie Island & Prudhoe \& Bray (1973) \\
\hline 204 & & & Derogenes varicus & Lepidonotothen squamifrons & Ob Bank & Parukhin \& Lyadov (1981) \\
\hline 205 & DIGENEA & & Derogenes varicus & Lepidonotothen squamifrons & Kerguelen Subregion & Parukhin \& Lyadov (1982) \\
\hline 206 & & & Derogenes varicus & Lepidonotothen squamifrons & Ob Bank & Parukhin \& Lyadov (1982) \\
\hline 207 & & & Derogenes varicus & Lepidonotothen squamifrons & Ob Bank & Parukhin (1986) \\
\hline 208 & & & Derogenes varicus & Micromesistius australis & South Western Atlantic & Gaevskaya \& Kovaleva (1978) \\
\hline 209 & & & Derogenes varicus & Merluccius hubbsi & Patagonian Shelf & Reimer \& Jessen (1974) \\
\hline
\end{tabular}




\begin{tabular}{|c|c|c|c|c|c|c|}
\hline Index & Phylum & Family & Species & Host & Location & References \\
\hline 210 & & & Derogenes varicus & Macruronus magellanicus & South Western Atlantic & Gaevskaya \& Kovaleva (1978) \\
\hline 211 & & & Derogenes varicus & Nototheniidae & Kerguelen Subregion & Parukhin \& Lyadov (1982) \\
\hline 212 & & & Derogenes varicus & Notothenia rossii & Ob Bank & Parukhin (1986) \\
\hline 213 & & & Derogenes varicus & Zanclorhynchus spinifer & A.A.E. & Prudhoe \& Bray (1973) \\
\hline 214 & & & Derogenes varicus & Zanclorhynchus spinifer & Macquarie Island & Prudhoe \& Bray (1973) \\
\hline 215 & & & Derogenes varicus & Zanclorhynchus spinifer & $54^{\circ} 28^{\prime} \mathrm{S} 158^{\circ} 53^{\prime} \mathrm{E}$ & Prudhoe \& Bray (1973) \\
\hline 216 & & & Derogenes varicus & Zanclorhynchus spinifer & $54^{\circ} 42^{\prime} 30^{\prime \prime S} 158^{\circ} 54^{\prime} 30^{\prime \prime} \mathrm{E}$ & Prudhoe \& Bray (1973) \\
\hline 217 & & & Derogenes varicus & Zanclorhynchus spinifer & $\begin{array}{l}\text { Off Lusitania Bay, } \\
\text { Macquarie Island }\end{array}$ & Prudhoe \& Bray (1973) \\
\hline 218 & & & Derogenes varicus & HOST NAME NOT MENTIONED & Patagonian Shelf & Lyadov (1985) \\
\hline 219 & & & Derogenes varicus & HOST NAME NOT MENTIONED & Glacial Subregion & Lyadov (1985) \\
\hline 220 & & & Derogenes varicus & HOST NAME NOT MENTIONED & Kerguelen Subregion & Lyadov (1985) \\
\hline 221 & & & $\begin{array}{c}\text { Gonocerca crassa validity doubtful; } \\
\text { probably G. phycidis }\end{array}$ & blank & blank & Manter (1934) \\
\hline 222 & & & $\begin{array}{c}\text { Gonocerca crassa validity doubtful; } \\
\text { probably G. phycidis }\end{array}$ & Dissostichus eleginoides & Falkland-Patagonian Shelf & Gaevskaya et al. (1990) \\
\hline 223 & & & $\begin{array}{c}\text { Gonocerca crassa validity doubtful; } \\
\text { probably G. phycidis }\end{array}$ & Merluccius hubbsi & South Western Atlantic & Gaevskaya \& Kovaleva (1978) \\
\hline 224 & & & $\begin{array}{c}\text { Gonocerca crassa validity doubtful; } \\
\text { probably G. phycidis }\end{array}$ & Salilota australis & South Western Atlantic & Gaevskaya \& Kovaleva (1978) \\
\hline 225 & & & Gonocerca haedrichi & blank & blank & Campbell \& Munroe (1977) \\
\hline 226 & & & Gonocerca haedrichi & Macrourus carinatus & $\begin{array}{l}\text { Falkland (Malvinas) } \\
\text { Islands }\end{array}$ & Gaevskaya \& Rodyuk (1988) \\
\hline 227 & & & Gonocerca medius & blank & blank & Lyadov (1985) \\
\hline 228 & & & Gonocerca medius & HOST NAME NOT MENTIONED & Kerguelen Subregion & Lyadov (1985) \\
\hline 229 & & Derogenidae & Gonocerca muraenolepisi & blank & blank & Parukhin \& Lyadov (1981) \\
\hline 230 & & & Gonocerca muraenolepisi & Chaenichthys rhinoceratus & Kerguelen Subregion & Parukhin \& Lyadov (1981) \\
\hline 231 & & & Gonocerca muraenolepisi & Chaenichthys rhinoceratus & Kerguelen Subregion & Lyadov et al. (1981) \\
\hline 232 & DIGENEA & & Gonocerca muraenolepisi & Dissostichus eleginoides & Ob Bank & Parukhin \& Lyadov (1981) \\
\hline 233 & & & Gonocerca muraenolepisi & Dissostichus eleginoides & Lena Bank & Parukhin \& Lyadov (1981) \\
\hline 234 & & & Gonocerca muraenolepisi & Lepidonotothen mizops & Kerguelen Subregion & Parukhin \& Lyadov (1981) \\
\hline 235 & & & Gonocerca muraenolepisi & Lepidonotothen mizops & Kerguelen Subregion & Parukhin \& Lyadov (1982) \\
\hline
\end{tabular}




\begin{tabular}{|c|c|c|c|c|c|c|}
\hline Index & Phylum & Family & Species & Host & Location & References \\
\hline 236 & & & Gonocerca muraenolepisi & Lepidonotothen squamifrons & Skif Bank & Parukhin \& Lyadov (1981) \\
\hline 237 & & & Gonocerca muraenolepisi & Lepidonotothen squamifrons & Ob Bank & Parukhin \& Lyadov (1981) \\
\hline 238 & & & Gonocerca muraenolepisi & Lepidonotothen squamifrons & Lena Bank & Parukhin \& Lyadov (1981) \\
\hline 239 & & & Gonocerca muraenolepisi & Nototheniidae & Kerguelen Subregion & Parukhin \& Lyadov (1982) \\
\hline 240 & & & Gonocerca muraenolepisi & Notothenia rossii & Heard Island & Parukhin \& Lyadov (1981) \\
\hline 241 & & & Gonocerca muraenolepisi & Notothenia rossii & Skif Bank & Parukhin \& Lyadov (1981) \\
\hline 242 & & & Gonocerca muraenolepisi & Notothenia rossii & Ob Bank & Parukhin \& Lyadov (1981) \\
\hline 243 & & & Gonocerca muraenolepisi & HOST NAME NOT MENTIONED & Kerguelen Subregion & Lyadov (1985) \\
\hline 244 & & & Gonocerca phycidis & blank & blank & Manter (1925) \\
\hline 245 & & & Gonocerca phycidis & Chaenocephalus aceratus & South Shetland Islands & Zdzitowiecki (1979) \\
\hline 246 & & & Gonocerca phycidis & Chaenocephalus aceratus & South Georgia Island & Zdzitowiecki (1991) \\
\hline 247 & & & Gonocerca phycidis & Chaenocephalus aceratus & South Georgia Island & Parukhin \& Lyadov (1981) \\
\hline 248 & & & Gonocerca phycidis & Chaenocephalus aceratus & South Shetland Islands & Zdzitowiecki (2002a) \\
\hline 249 & & & Gonocerca phycidis & Chaenocephalus aceratus & South Shetland Islands & Palm et al. (2007) \\
\hline 250 & & & Gonocerca phycidis & Chaenichthys rhinoceratus & Kerguelen Subregion & Lyadov et al. (1981) \\
\hline 251 & & & Gonocerca phycidis & Chaenichthys rhinoceratus & Kerguelen Subregion & Parukhin \& Lyadov (1981) \\
\hline 252 & & & Gonocerca phycidis & Chaenichthys velifer & Kerguelen Subregion & Lyadov et al. (1981) \\
\hline 253 & & & Gonocerca phycidis & Chaenichthys velifer & Kerguelen Subregion & Parukhin \& Lyadov (1981) \\
\hline 254 & & & Gonocerca phycidis & Champsocephalus gunnari & $49^{\circ} 28^{\prime} \mathrm{S} 70^{\circ} 33^{\prime} \mathrm{E}$ & Prudhoe \& Bray (1973) \\
\hline 255 & & & Gonocerca phycidis & Champsocephalus gunnari & $\begin{array}{l}\text { Off Entrance To Royal } \\
\text { Sound (Kerguelen } \\
\text { Subregion) }\end{array}$ & Prudhoe \& Bray (1973) \\
\hline 256 & & & Gonocerca phycidis & Champsocephalus gunnari & South Shetland Islands & Zdzitowiecki (1991) \\
\hline 257 & & & Gonocerca phycidis & Chionodraco rastrospinosus & South Shetland Islands & Zdzitowiecki (1991) \\
\hline 258 & \multirow{4}{*}{ DIGENEA } & Derogenidae & Gonocerca phycidis & Cryodraco antarcticus & South Shetland Islands & Zdzitowiecki (1991) \\
\hline 259 & & & Gonocerca phycidis & Cryodraco antarcticus & South Shetland Islands & Zdzitowiecki (2002a) \\
\hline 260 & & & Gonocerca phycidis & Dissostichus eleginoides & South Western Atlantic & Gaevskaya \& Kovaleva (1978) \\
\hline 261 & & & Gonocerca phycidis & Dissostichus eleginoides & Kerguelen Subregion & Parukhin \& Lyadov (1981) \\
\hline
\end{tabular}




\begin{tabular}{|c|c|c|c|c|c|c|}
\hline Index & Phylum & Family & Species & Host & Location & References \\
\hline 262 & & & Gonocerca phycidis & Dissostichus eleginoides & Ob Bank & Parukhin \& Lyadov (1982) \\
\hline 263 & & & Gonocerca phycidis & Dissostichus eleginoides & Lena Bank & Parukhin \& Lyadov (1982) \\
\hline 264 & & & Gonocerca phycidis & Dissostichus eleginoides & Shag Rocks & Parukhin \& Lyadov (1982) \\
\hline 265 & & & Gonocerca phycidis & Dissostichus eleginoides & Ob Bank & Parukhin (1986) \\
\hline 266 & & & Gonocerca phycidis & Dissostichus eleginoides & Lena Bank & Parukhin (1986) \\
\hline 267 & & & Gonocerca phycidis & Dissostichus eleginoides & South Shetland Islands & Zdzitowiecki (1991) \\
\hline 268 & & & Gonocerca phycidis & Dissostichus eleginoides & South Georgia Island & Zdzitowiecki (1991) \\
\hline 269 & & & Gonocerca phycidis & Dissostichus eleginoides & Falkland-Patagonian Shelf & Gaevskaya et al. (1990) \\
\hline 270 & & & Gonocerca phycidis & Dissostichus eleginoides & South Georgia Island & Gaevskaya et al. (1990) \\
\hline 271 & & & Gonocerca phycidis & Dissostichus eleginoides & Shag Rocks & Brickle et al. (2005) \\
\hline 272 & & & Gonocerca phycidis & Dissostichus eleginoides & South Georgia Island & Brickle et al. (2005) \\
\hline 273 & & & Gonocerca phycidis & Dissostichus eleginoides & Prince Edward Island & Brickle et al. (2005) \\
\hline 274 & & & Gonocerca phycidis & Dissostichus eleginoides & Heard Island & Brickle et al. (2005) \\
\hline 275 & & & Gonocerca phycidis & Dissostichus eleginoides & Macquarie Island & Brickle et al. (2005) \\
\hline 276 & & & Gonocerca phycidis & Dissostichus eleginoides & Falkland Islands & Brickle et al. (2006) \\
\hline 277 & & & Gonocerca phycidis & Gobionotothen acuta & Kerguelen Subregion & Parukhin \& Lyadov (1981) \\
\hline 278 & & & Gonocerca phycidis & Gobionotothen acuta & Kerguelen Subregion & Parukhin \& Lyadov (1982) \\
\hline 279 & & & Gonocerca phycidis & Gobionotothen gibberifrons & South Georgia Island & Parukhin \& Lyadov (1981) \\
\hline 280 & & & Gonocerca phycidis & Harpagifer georgianus & Admiralty Bay & Zdzitowiecki \& Zadrozny (1999) \\
\hline 281 & & & Gonocerca phycidis & Lepidonotothen mizops & Kerguelen Subregion & Parukhin \& Lyadov (1981) \\
\hline 282 & & & Gonocerca phycidis & Lepidonotothen mizops & Kerguelen Subregion & Parukhin \& Lyadov (1982) \\
\hline 283 & & & Gonocerca phycidis & Lepidonotothen squamifrons & Kerguelen Subregion & Parukhin \& Lyadov (1982) \\
\hline 284 & & & Gonocerca phycidis & Lepidonotothen squamifrons & Skif Bank & Parukhin \& Lyadov (1981) \\
\hline 285 & & & Gonocerca phycidis & Lepidonotothen squamifrons & Ob Bank & Parukhin \& Lyadov (1982) \\
\hline 286 & & & Gonocerca phycidis & Lepidonotothen squamifrons & Lena Bank & Parukhin \& Lyadov (1983) \\
\hline 287 & & Derogenidae & Gonocerca phycidis & Lindbergichthys nudifrons & South Georgia Island & Zdzitowiecki (1991) \\
\hline 288 & DIGENEA & & Gonocerca phycidis & Lindbergichthys nudifrons & Vernadsky Station & $\begin{array}{l}\text { Laskowski \& Zdzitowiecki } \\
\text { (2005) }\end{array}$ \\
\hline
\end{tabular}




\begin{tabular}{|c|c|c|c|c|c|c|}
\hline Index & Phylum & Family & Species & Host & Location & References \\
\hline 289 & & & Gonocerca phycidis & Macrourus carinatus & $\begin{array}{l}\text { Falkland (Malvinas) } \\
\text { Islands }\end{array}$ & Gaevskaya \& Rodyuk (1988) \\
\hline 290 & & & Gonocerca phycidis & Macrourus holotrachys & North Scotia Ridge & Zdzitowiecki \& Cielecka (1998) \\
\hline 291 & & & Gonocerca phycidis & Muraenolepis microps & South Georgia Island & Zdzitowiecki (1991) \\
\hline 292 & & & Gonocerca phycidis & Macrourus whitsoni & Weddell Sea & Zdzitowiecki \& Cielecka (1997a) \\
\hline 293 & & & Gonocerca phycidis & Macrourus whitsoni & Weddell Sea & Zdzitowiecki (2003) \\
\hline 294 & & & Gonocerca phycidis & Nematonurus armatus & A.A.E. & Prudhoe \& Bray (1973) \\
\hline 295 & & & Gonocerca phycidis & Nematonurus armatus & $\begin{array}{l}140 \text { miles West of } \\
\text { Kangaroo Island }\end{array}$ & Prudhoe \& Bray (1973) \\
\hline 296 & & & Gonocerca phycidis & Nematonurus armatus & South Australia & Prudhoe \& Bray (1973) \\
\hline 297 & & & Gonocerca phycidis & Nototheniidae & Kerguelen Subregion & Parukhin \& Lyadov (1982) \\
\hline 298 & & & Gonocerca phycidis & Notothenia coriiceps & $\begin{array}{c}\text { Potter Cove (King George } \\
\text { Island) }\end{array}$ & Palm et.al. (1998) \\
\hline 299 & & & Gonocerca phycidis & Notothenia coriiceps & King George Island & Palm et.al. (1998) \\
\hline 300 & & & Gonocerca phycidis & Notothenia coriiceps & Adelie Land & Zdzitowiecki et.al. (1998) \\
\hline 301 & & & Gonocerca phycidis & Notothenia coriiceps & Admiralty Bay & $\begin{array}{l}\text { Zdzitowiecki \& Laskowski } \\
\text { (2004) }\end{array}$ \\
\hline 302 & & & Gonocerca phycidis & Notothenia coriiceps & Vernadsky Station & $\begin{array}{c}\text { Zdzitowiecki \& Laskowski } \\
\text { (2004) }\end{array}$ \\
\hline 303 & & & Gonocerca phycidis & Notothenia gibberifrons & South Shetland Islands & Zdzitowiecki (1991) \\
\hline 304 & & & Gonocerca phycidis & Notothenia gibberifrons & South Georgia Island & Zdzitowiecki (1991) \\
\hline 305 & & & Gonocerca phycidis & Notothenia rossii & Kerguelen Subregion & Parukhin \& Lyadov (1981) \\
\hline 306 & & & Gonocerca phycidis & Notothenia rossii & Heard Island & Parukhin \& Lyadov (1982) \\
\hline 307 & & & Gonocerca phycidis & Notothenia rossii & Skif Bank & Parukhin \& Lyadov (1982) \\
\hline 308 & & & Gonocerca phycidis & Notothenia rossii & Ob Bank & Parukhin \& Lyadov (1982) \\
\hline 309 & & & Gonocerca phycidis & Notothenia rossii & South Georgia Island & Parukhin \& Lyadov (1982) \\
\hline 310 & & & Gonocerca phycidis & Notothenia rossii & Ob Bank & Parukhin (1986) \\
\hline 311 & & & Gonocerca phycidis & Notothenia rossii & Lena Bank & Parukhin (1986) \\
\hline 312 & & & Gonocerca phycidis & Notothenia rossii & South Georgia Island & Zdzitowiecki (1991) \\
\hline 313 & & & Gonocerca phycidis & Nototheniops larseni & Lena Bank & Parukhin (1986) \\
\hline 314 & & & Gonocerca phycidis & Nototheniops larseni & Ob Bank & Parukhin (1986) \\
\hline
\end{tabular}




\begin{tabular}{|c|c|c|c|c|c|c|}
\hline Index & Phylum & Family & Species & Host & Location & References \\
\hline 315 & & Derogenidae & Gonocerca phycidis & Pagothenia hansoni & South Georgia Island & Zdzitowiecki (1991) \\
\hline 316 & & & Gonocerca phycidis & Parachaenichthys georgianus & South Shetland Islands & Zdzitowiecki (1979) \\
\hline 317 & & & Gonocerca phycidis & Parachaenichthys georgianus & South Georgia Island & Zdzitowiecki (1991) \\
\hline 318 & & & Gonocerca phycidis & Pseudochaenichthys georgianus & South Georgia Island & Zdzitowiecki (1979) \\
\hline 319 & & & Gonocerca phycidis & Pseudochaenichthys georgianus & South Georgia Island & Zdzitowiecki (1991) \\
\hline 320 & & & Gonocerca phycidis & Pseudochaenichthys georgianus & South Shetland Islands & Zdzitowiecki (2002a) \\
\hline 321 & & & Gonocerca phycidis & Stromateus maculatus & South Western Atlantic & Gaevskaya \& Kovaleva (1978) \\
\hline 322 & & & Gonocerca phycidis & Trematomus loennbergi & Weddell Sea & Zdzitowiecki (2002b) \\
\hline 323 & & & Gonocerca phycidis & Trematomus hansoni & Adelie Land & Zdzitowiecki (2001b) \\
\hline 324 & & & Gonocerca phycidis & HOST NAME NOT MENTIONED & Patagonian Shelf & Lyadov (1985) \\
\hline 325 & & & Gonocerca phycidis & HOST NAME NOT MENTIONED & Glacial Subregion & Lyadov (1986) \\
\hline 326 & & & Gonocerca phycidis & HOST NAME NOT MENTIONED & Kerguelen Subregion & Lyadov (1987) \\
\hline 327 & & & Gonocerca phycidis & HOST NAME NOT MENTIONED & South Australia & Lyadov (1988) \\
\hline 328 & DIGENEA & & Gonocerca taeniata & blank & blank & $\begin{array}{l}\text { Gaevskaya \& Rodjuk (1983) } \\
\text { (probably valid) }\end{array}$ \\
\hline 329 & & & Gonocerca taeniata & Dissostichus eleginoides & Falkland-Patagonian Shelf & Gaevskaya et al. (1990) \\
\hline 330 & & Fellodistomidae & Benthotrema melanostigmi & blank & blank & Parukhin \& Lyadov (1979) \\
\hline 331 & & & Benthotrema melanostigmi & HOST NAME NOT MENTIONED & Kerguelen Subregion & Lyadov (1985) \\
\hline 332 & & & Fellodistomum sp. & blank & blank & Stafford (1904) \\
\hline 333 & & & Fellodistomum sp. & Artedidraco mirus & South Georgia Island & Zdzitowiecki (1991) \\
\hline 334 & & & Lomasoma kergeleni & blank & blank & Parukhin \& Lyadov (1979) \\
\hline 335 & & & Lomasoma kergeleni & HOST NAME NOT MENTIONED & Kerguelen Subregion & Lyadov (1985) \\
\hline 336 & & & Monascus typicus & blank & blank & Odhner (1911) \\
\hline 337 & & & Monascus typicus & Stromateus maculatus & South Western Atlantic & Gaevskaya \& Kovaleva (1978) \\
\hline 338 & & & Steringophorus sp. indet. & blank & blank & Odhner (1905) \\
\hline 339 & & & Steringophorus sp. indet. & Zanclorhynchus spinifer & $54^{\circ} 42^{\prime} 30^{\prime \prime} \mathrm{S} 158^{\circ} 54^{\prime} 30^{\prime \prime} \mathrm{E}$ & Prudhoe \& Bray (1971) \\
\hline 340 & & & Steringophorus sp. indet. & Zanclorhynchus spinifer & $\begin{array}{l}\text { Off Lusitania Bay, } \\
\text { Macquarie Island }\end{array}$ & Prudhoe \& Bray (1971) \\
\hline 341 & & & Steringophorus arntzi & blank & blank & Zdzitowiecki (1997) \\
\hline
\end{tabular}




\begin{tabular}{|c|c|c|c|c|c|c|}
\hline Index & Phylum & Family & Species & Host & Location & References \\
\hline 342 & & & Steringophorus arntzi & Artedidroconidae & Weddell Sea & Zdzitowiecki (2002c) \\
\hline 343 & & & Steringophorus arntzi & Dolloidraco longedorsalis & Weddell Sea & Zdzitowiecki (2002c) \\
\hline 344 & \multirow{25}{*}{ DIGENEA } & Fellodistomidae & Steringophorus arntzi & Bathydraco marri & Weddell Sea & Zdzitowiecki (2002d) \\
\hline 345 & & & Steringophorus arntzi & Bathydraco marri & Weddell Sea & Zdzitowiecki (1997b) \\
\hline 346 & & & Steringophorus arntzi & Dolloidraco largedorsalis & Weddell Sea & Zdzitowiecki (1997b) \\
\hline 347 & & & Steringophorus liparidis & blank & blank & Zdzitowiecki (1997) \\
\hline 348 & & & Steringophorus liparidis & Paraliparis antarcticus & Weddell Sea & Zdzitowiecki (1997b) \\
\hline 349 & & & Steringophorus liparidis & Paraliparis antarcticus & Weddell Sea & Zdzitowiecki (2003) \\
\hline 350 & & Hemiuridae & Hemiuridae sp. indet. & Bathylagus antarcticus & $65^{\circ} 10^{\prime} \mathrm{S} 109^{\circ} 32^{\prime} \mathrm{E}$ & Prudhoe \& Bray (1973) \\
\hline 351 & & & Aphanurus sp. & blank & blank & Monticelli (1891) \\
\hline 352 & & & Aphanurus sp. & Ophthalmolycus amberensis & Weddell Sea & Zdzitowiecki (2003) \\
\hline 353 & & & Boreascotia megavesicula & blank & blank & Bray \& Zdzitowiecki (2000) \\
\hline 354 & & & Boreascotia megavesicula & Lepidonotothen macrophthalma & North Scotia Ridge & Bray \& Zdzitowiecki (2000) \\
\hline 355 & & & Brachyphallus crenatus & blank & blank & Rudolphi (1802) \\
\hline 356 & & & Brachyphallus crenatus & Dissostichus eleginoides & Falkland-Patagonian Shelf & Gaevskaya et al. (1990) \\
\hline 357 & & & Brachyphallus crenatus & Macrourus carinatus & $\begin{array}{l}\text { Falkland (Malvinas) } \\
\text { Islands }\end{array}$ & Gaevskaya \& Rodyuk (1988) \\
\hline 358 & & & Brachyphallus parvus & blank & blank & Collin (1931) \\
\hline 359 & & & Brachyphallus parvus & Macruronus magellanicus & South Western Atlantic & Gaevskaya \& Kovaleva (1978) \\
\hline 360 & & & Dissosaccus gravidus & blank & blank & Looss (1907) \\
\hline 361 & & & Dissosaccus gravidus & Merluccius hubbsi & Patagonian Shelf & Reimer \& Jessen (1974) \\
\hline 362 & & & Elytrophalloides merluccii & blank & blank & Szidat ???? \\
\hline 363 & & & Elytrophalloides merluccii & Merluccius hubbsi & Patagonian Shelf & Reimer \& Jessen (1974) \\
\hline 364 & & & Elytrophalloides oatesi & blank & blank & Leiper \& Atkinson (1914) \\
\hline 365 & & & Elytrophalloides oatesi & blank & blank & Szidat \& Graefe (1967) \\
\hline 366 & & & Elytrophalloides oatesi & Artedidraconidae & Weddell Sea & Zdzitowiecki (2002c) \\
\hline 367 & & & Elytrophalloides oatesi & Artedidraco mirus & South Georgia Island & Zdzitowiecki (1991) \\
\hline 368 & & & Elytrophalloides oatesi & Artedidraco skottsbergi & Weddell Sea & Zdzitowiecki (2002c) \\
\hline
\end{tabular}




\begin{tabular}{|c|c|c|c|c|c|c|}
\hline Index & Phylum & Family & Species & Host & Location & References \\
\hline 369 & & & Elytrophalloides oatesi & Chaenocephalus aceratus & South Shetland Islands & Zdzitowiecki (1979) \\
\hline 370 & & & Elytrophalloides oatesi & Chaenocephalus aceratus & South Georgia Island & Zdzitowiecki (1979) \\
\hline 371 & & & Elytrophalloides oatesi & Chaenocephalus aceratus & South Georgia Island & Zdzitowiecki (1991) \\
\hline 372 & & & Elytrophalloides oatesi & Champsocephalus gunnari & South Georgia Island & Zdzitowiecki (1979) \\
\hline 373 & & Hemiuridae & Elytrophalloides oatesi & Channichthys rhinoceratus & Kerguelen Subregion & Lyadov et al. (1981) \\
\hline 374 & & & Elytrophalloides oatesi & Channichthys rhinoceratus & Kerguelen Subregion & Parukhin \& Lyadov (1981) \\
\hline 375 & & & Elytrophalloides oatesi & Chionodraco hamatus & Adelie Land & Zdzitowiecki et.al. (1998) \\
\hline 376 & & & Elytrophalloides oatesi & Chionodraco hamatus & Adelie Land & Zdzitowiecki (2001b) \\
\hline 377 & & & Elytrophalloides oatesi & Chionodraco kathleenae & A.A.E. & Prudhoe \& Bray (1973) \\
\hline 378 & & & Elytrophalloides oatesi & Chionodraco kathleenae & $65^{\circ} 6^{\prime} \mathrm{S} 96^{\circ} 14^{\prime} \mathrm{E}$ & Prudhoe \& Bray (1973) \\
\hline 379 & & & Elytrophalloides oatesi & Chionodraco rastrospinosus & South Shetland Islands & Zdzitowiecki (1991) \\
\hline 380 & & & Elytrophalloides oatesi & Cryodraco antarcticus & $65^{\circ} 42^{\prime} \mathrm{S} 92^{\circ} 10^{\prime} \mathrm{E}$ & Prudhoe \& Bray (1973) \\
\hline 381 & & & Elytrophalloides oatesi & Cryodraco antarcticus & South Shetland Islands & Zdzitowiecki (1991) \\
\hline 382 & & & Elytrophalloides oatesi & Cryodraco antarcticus & Ross Sea & Zdzitowiecki (2002a) \\
\hline 383 & & & Elytrophalloides oatesi & Cygnodraco mawsoni & $66^{\circ} 45^{\prime} \mathrm{S} 62^{\circ} 03^{\prime} \mathrm{E}$ & Prudhoe \& Bray (1973) \\
\hline 384 & DIGENEA & & Elytrophalloides oatesi & Cygnodraco mawsoni & Ross Sea & Laskowski et.al. (2005) \\
\hline 385 & & & Elytrophalloides oatesi & Dissostichus eleginoides & Kerguelen Subregion & Parukhin \& Lyadov (1981) \\
\hline 386 & & & Elytrophalloides oatesi & Dissostichus eleginoides & Crozet Islands & Parukhin \& Lyadov (1982) \\
\hline 387 & & & Elytrophalloides oatesi & Dissostichus eleginoides & Ob Bank & Parukhin \& Lyadov (1983) \\
\hline 388 & & & Elytrophalloides oatesi & Dissostichus eleginoides & Kerguelen Subregion & Parukhin \& Lyadov (1982) \\
\hline 389 & & & Elytrophalloides oatesi & Dissostichus eleginoides & Ob Bank & Parukhin \& Lyadov (1983) \\
\hline 390 & & & Elytrophalloides oatesi & Dissostichus eleginoides & Skif Bank & Parukhin \& Lyadov (1984) \\
\hline 391 & & & Elytrophalloides oatesi & Dissostichus eleginoides & Ob Bank & Parukhin (1986) \\
\hline 392 & & & Elytrophalloides oatesi & Dissostichus eleginoides & Lena Bank & Parukhin (1986) \\
\hline 393 & & & Elytrophalloides oatesi & Dissostichus eleginoides & Falkland-Patagonian Shelf & Gaevskaya et al. (1990) \\
\hline 394 & & & Elytrophalloides oatesi & Dissostichus eleginoides & South Georgia Island & Gaevskaya et al. (1990) \\
\hline 395 & & & Elytrophalloides oatesi & Dissostichus eleginoides & South Shetland Islands & Zdzitowiecki (1991) \\
\hline
\end{tabular}




\begin{tabular}{|c|c|c|c|c|c|c|}
\hline Index & Phylum & Family & Species & Host & Location & References \\
\hline 396 & & & Elytrophalloides oatesi & Dissostichus eleginoides & South Georgia Island & Zdzitowiecki (1991) \\
\hline 397 & & & Elytrophalloides oatesi & Dissostichus eleginoides & Shag Rocks & Brickle et al. (2005) \\
\hline 398 & & & Elytrophalloides oatesi & Dissostichus eleginoides & South Georgia Island & Brickle et al. (2005) \\
\hline 399 & & & Elytrophalloides oatesi & Dissostichus eleginoides & Prince Edward Island & Brickle et al. (2005) \\
\hline 400 & & & Elytrophalloides oatesi & Dissostichus eleginoides & Heard Island & Brickle et al. (2005) \\
\hline 401 & & & Elytrophalloides oatesi & Dissostichus eleginoides & Falkland Islands & Brickle et al. (2006) \\
\hline 402 & \multirow{20}{*}{ DIGENEA } & Hemiuridae & Elytrophalloides oatesi & Gobionotothen gibberifrons & South Georgia Island & Zdzitowiecki (1991) \\
\hline 403 & & & Elytrophalloides oatesi & Gymnodraco acuticeps & Ross Sea & Laskowski et.al. (2005) \\
\hline 404 & & & Elytrophalloides oatesi & Lepidonotothen mizops & Kerguelen Subregion & Prudhoe \& Bray (1973) \\
\hline 405 & & & Elytrophalloides oatesi & Lindbergichthys nudifrons & South Georgia Island & Zdzitowiecki (1991) \\
\hline 406 & & & Elytrophalloides oatesi & Lindbergichthys nudifrons & Vernadsky Station & $\begin{array}{l}\text { Laskowski \& Zdzitowiecki } \\
\qquad(2005)\end{array}$ \\
\hline 407 & & & Elytrophalloides oatesi & Lindbergichthys nudifrons & Admiralty Bay & $\begin{array}{l}\text { Laskowski \& Zdzitowiecki } \\
\text { (2005) }\end{array}$ \\
\hline 408 & & & Elytrophalloides oatesi & Lepidonotothen squamifrons & Ob Bank & Parukhin (1986) \\
\hline 409 & & & Elytrophalloides oatesi & Lepidonotothen squamifrons & Crozet Islands & Parukhin \& Lyadov (1981) \\
\hline 410 & & & Elytrophalloides oatesi & Lepidonotothen squamifrons & Crozet Islands & Parukhin \& Lyadov (1982) \\
\hline 411 & & & Elytrophalloides oatesi & Lepidonotothen squamifrons & South Georgia Island & Zdzitowiecki (1991) \\
\hline 412 & & & Elytrophalloides oatesi & Macrourus carinatus & $\begin{array}{l}\text { Falkland (Malvinas) } \\
\text { Islands }\end{array}$ & Gaevskaya \& Rodyuk (1988) \\
\hline 413 & & & Elytrophalloides oatesi & Muraenolepis microps & South Georgia Island & Zdzitowiecki (1991) \\
\hline 414 & & & Elytrophalloides oatesi & Nototheniidae & Kerguelen Subregion & Parukhin \& Lyadov (1982) \\
\hline 415 & & & Elytrophalloides oatesi & Notothenia coriiceps & South Shetland Islands & Zdzitowiecki (1979) \\
\hline 416 & & & Elytrophalloides oatesi & Notothenia coriiceps & $\begin{array}{c}\text { Potter Cove (King George } \\
\text { Island) }\end{array}$ & Palm et.al. (1998) \\
\hline 417 & & & Elytrophalloides oatesi & Notothenia coriiceps & King George Island & Palm et.al. (1998) \\
\hline 418 & & & Elytrophalloides oatesi & Notothenia coriiceps & Adelie Land & Zdzitowiecki et.al. (1998) \\
\hline 419 & & & Elytrophalloides oatesi & Notothenia coriiceps & Adelie Land & Zdzitowiecki (2001b) \\
\hline 420 & & & Elytrophalloides oatesi & Notothenia coriiceps & Admiralty Bay & $\begin{array}{c}\text { Zdzitowiecki \& Laskowski } \\
\text { (2004) }\end{array}$ \\
\hline 421 & & & Elytrophalloides oatesi & Notothenia coriiceps & Vernadsky Station & $\begin{array}{c}\text { Zdzitowiecki \& Laskowski } \\
\text { (2004) }\end{array}$ \\
\hline
\end{tabular}




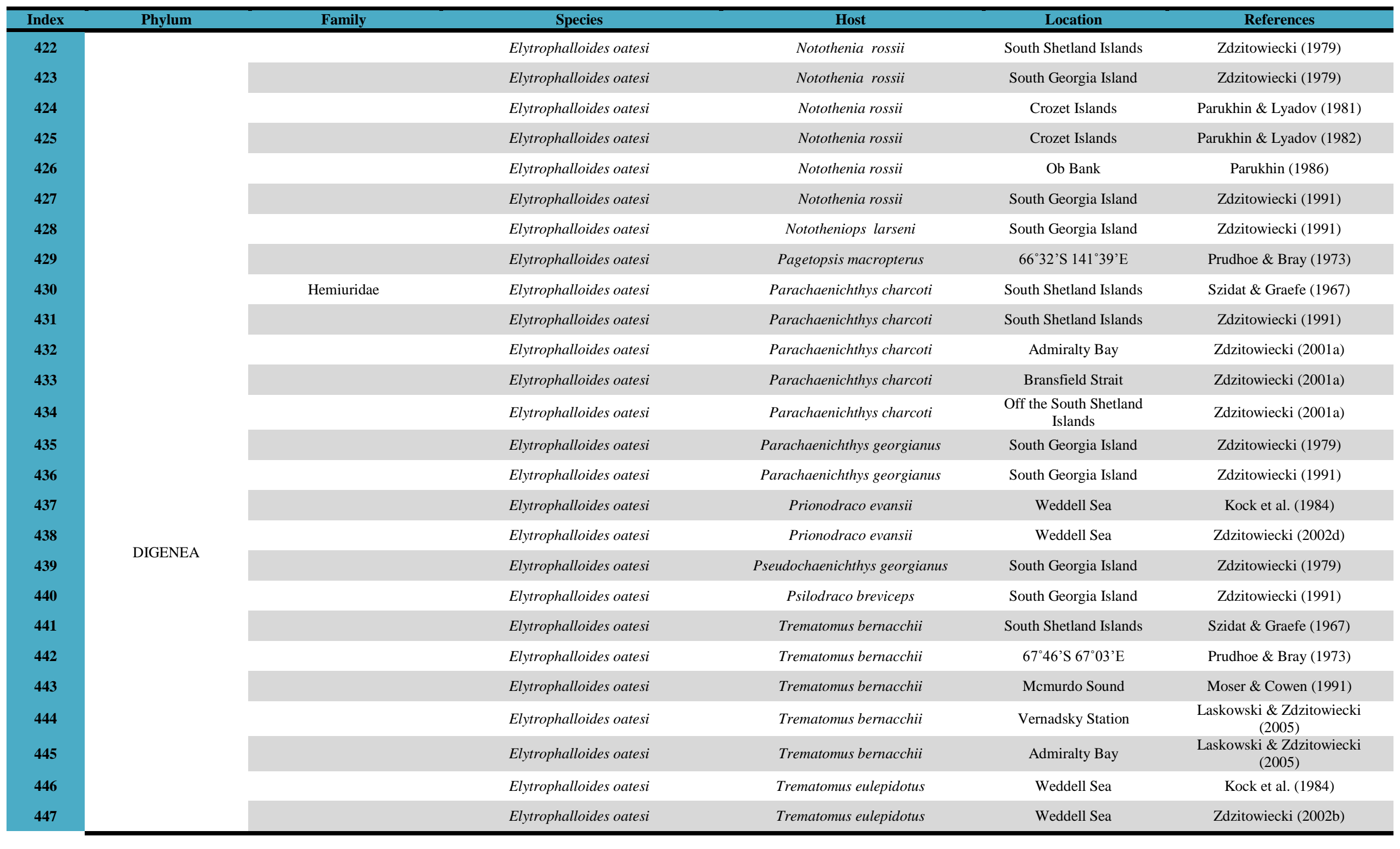




\begin{tabular}{|c|c|c|c|c|c|c|}
\hline Index & Phylum & Family & Species & Host & Location & References \\
\hline 448 & & & Elytrophalloides oatesi & Trematomus hansoni & South Georgia Island & Zdzitowiecki (1979) \\
\hline 449 & & & Elytrophalloides oatesi & Trematomus hansoni & South Georgia Island & Zdzitowiecki (1991) \\
\hline 450 & & & Elytrophalloides oatesi & Trematomus hansoni & Adelie Land & Zdzitowiecki (2001b) \\
\hline 451 & & & Elytrophalloides oatesi & Trematomus pennellii & $67^{\circ} 46^{\prime} \mathrm{S} 67^{\circ} 03^{\prime} \mathrm{E}$ & Prudhoe \& Bray (1973) \\
\hline 452 & & & Elytrophalloides oatesi & Trematomus lepidorhinus & $66^{\circ} 45^{\prime} \mathrm{S} 62^{\circ} 03^{\prime} \mathrm{E}$ & Prudhoe \& Bray (1973) \\
\hline 453 & & & Elytrophalloides oatesi & Trematomus lepidorhinus & Weddell Sea & Zdzitowiecki (2002b) \\
\hline 454 & & & Elytrophalloides oatesi & Trematomus newnesi & $66^{\circ} 45^{\prime} \mathrm{S} 64^{\circ} 52^{\prime} \mathrm{E}$ & Prudhoe \& Bray (1973) \\
\hline 455 & & & Elytrophalloides oatesi & Trematomus newnesi & Vernadsky Station & $\begin{array}{l}\text { Laskowski \& Zdzitowiecki } \\
\text { (2005) }\end{array}$ \\
\hline 456 & & & Elytrophalloides oatesi & Trematomus newnesi & $\begin{array}{l}\text { Adelie Land (Eastern } \\
\text { Antarctica) }\end{array}$ & Laskowski et.al. (2007) \\
\hline 457 & & & Elytrophalloides oatesi & Trematomus nicolai & Weddell Sea & Zdzitowiecki (2002b) \\
\hline 458 & & Hemiuridae & Elytrophalloides oatesi & Trematomus scotti & $67^{\circ} 03^{\prime} \mathrm{S} 74^{\circ} 29^{\prime} \mathrm{E}$ & Prudhoe \& Bray (1973) \\
\hline 459 & & & Elytrophalloides oatesi & Trematomus scotti & Weddell Sea & Zdzitowiecki (2002b) \\
\hline 460 & & & Elytrophalloides oatesi & Upeneichthys porosus & $35^{\circ} 18^{\prime} \mathrm{S} 118^{\circ} 15^{\prime} \mathrm{E}$ & Prudhoe \& Bray (1973) \\
\hline 461 & & & Elytrophalloides oatesi & Upeneichthys porosus & Continental Shelf & Prudhoe \& Bray (1973) \\
\hline 462 & & & Elytrophalloides oatesi & Upeneichthys porosus & East Of Albany & Prudhoe \& Bray (1973) \\
\hline 463 & & & Elytrophalloides oatesi & Upeneichthys porosus & Western Australia & Prudhoe \& Bray (1973) \\
\hline 464 & & & Elytrophalloides oatesi & HOST NAME NOT MENTIONED & Patagonian Shelf & Lyadov (1985) \\
\hline 465 & & & Elytrophalloides oatesi & HOST NAME NOT MENTIONED & Glacial Subregion & Lyadov (1985) \\
\hline 466 & DIGENEA & & Elytrophalloides oatesi & HOST NAME NOT MENTIONED & Kerguelen Subregion & Lyadov (1985) \\
\hline 467 & & & Elytrophalloides oatesi & HOST NAME NOT MENTIONED & South Australia & Lyadov (1985) \\
\hline 468 & & & Genolinea bowersi & blank & blank & Leiper \& Atkinson (1914) \\
\hline 469 & & & Genolinea bowersi & Artedidraconidae & Weddell Sea & Zdzitowiecki (2002c) \\
\hline 470 & & & Genolinea bowersi & Artedidraco loennbergi & Weddell Sea & Zdzitowiecki (2002c) \\
\hline 471 & & & Genolinea bowersi & Artedidraco mirus & South Georgia Island & Zdzitowiecki (1991) \\
\hline 472 & & & Genolinea bowersi & Artedidraco orianae & Weddell Sea & Zdzitowiecki (2002c) \\
\hline 473 & & & Genolinea bowersi & Artedidraco shackletoni & A.A.E. & Prudhoe \& Bray (1973) \\
\hline 474 & & & Genolinea bowersi & Artedidraco shackletoni & $65^{\circ} 42^{\prime} \mathrm{S} 92^{\circ} 10^{\prime} \mathrm{E}$ & Prudhoe \& Bray (1973) \\
\hline
\end{tabular}




\begin{tabular}{|c|c|c|c|c|c|c|}
\hline Index & Phylum & Family & Species & Host & Location & References \\
\hline 475 & & & Genolinea bowersi & Chaenocephalus aceratus & South Shetland Islands & Zdzitowiecki (1979) \\
\hline 476 & & & Genolinea bowersi & Chaenocephalus aceratus & South Georgia Island & Zdzitowiecki (1991) \\
\hline 477 & & & Genolinea bowersi & Chaenocephalus aceratus & Signy Island & Zdzitowiecki et al. (1997) \\
\hline 478 & & & Genolinea bowersi & Chaenocephalus aceratus & Orkny Islands & Zdzitowiecki et al. (1997) \\
\hline 479 & & & Genolinea bowersi & Chaenocephalus aceratus & Admiralty Bay & Zdzitowiecki (2002a) \\
\hline 480 & & & Genolinea bowersi & Chionodraco hamatus & Adelie Land & Zdzitowiecki et.al. (1998) \\
\hline 481 & & & Genolinea bowersi & Chionodraco hamatus & Adelie Land & Zdzitowiecki (2001b) \\
\hline 482 & & & Genolinea bowersi & Chiondraco rastrospinosus & Admiralty Bay & Zdzitowiecki (2002a) \\
\hline 483 & & & Genolinea bowersi & Cryodraco antarcticus & Admiralty Bay & Zdzitowiecki (2002a) \\
\hline 484 & & & Genolinea bowersi & Cygnodraco mawsoni & Ross Sea & Laskowski et.al. (2005) \\
\hline 485 & & & Genolinea bowersi & Dolloidraco langedorsalis & Weddell Sea & Zdzitowiecki (2002c) \\
\hline 486 & & & Genolinea bowersi & Gobionotothen gibberifrons & South Shetland Islands & Zdzitowiecki (1979) \\
\hline 487 & \multirow{14}{*}{ DIGENEA } & Hemiuridae & Genolinea bowersi & Gobionotothen gibberifrons & South Georgia Island & Zdzitowiecki (1991) \\
\hline 488 & & & Genolinea bowersi & Gymnodraco acuticeps & Ross Sea & Laskowski et.al. (2005) \\
\hline 489 & & & Genolinea bowersi & Harpagifer bispinis & Port Jeanne d'Arc & Prudhoe \& Bray (1973) \\
\hline 490 & & & Genolinea bowersi & Harpagifer bispinis & Royal Sound & Prudhoe \& Bray (1973) \\
\hline 491 & & & Genolinea bowersi & Harpagifer bispinis & Kerguelen Subregion & Prudhoe \& Bray (1973) \\
\hline 492 & & & Genolinea bowersi & Harpagifer georgianus & Admiralty Bay & Zdzitowiecki \& Zadrozny (1999) \\
\hline 493 & & & Genolinea bowersi & Lepidonotothen mizops & $\begin{array}{c}\text { Bras Bolinder (Kerguelen } \\
\text { Subregion) }\end{array}$ & Prudhoe \& Bray (1973) \\
\hline 494 & & & Genolinea bowersi & Lepidonotothen mizops & $\begin{array}{l}\text { Near Mouth of Peace } \\
\text { River }\end{array}$ & Prudhoe \& Bray (1973) \\
\hline 495 & & & Genolinea bowersi & Lindbergichthys nudifrons & South Shetland Islands & Zdzitowiecki (1979) \\
\hline 496 & & & Genolinea bowersi & Linbergichthys nudifrons & South Georgia Island & Zdzitowiecki (1991) \\
\hline 497 & & & Genolinea bowersi & Lindbergichthys nudifrons & Vernadsky Station & $\begin{array}{l}\text { Laskowski \& Zdzitowiecki } \\
\qquad(2005)\end{array}$ \\
\hline 498 & & & Genolinea bowersi & Lindbergichthys nudifrons & Admiralty Bay & $\begin{array}{c}\text { Laskowski \& Zdzitowiecki } \\
\text { (2005) }\end{array}$ \\
\hline 499 & & & Genolinea bowersi & Lindbergichthys nudifrons & Vernadsky Station & $\begin{array}{l}\text { Laskowski \& Zdzitowiecki } \\
\text { (2005) }\end{array}$ \\
\hline 500 & & & Genolinea bowersi & Macrourus carinatus & $\begin{array}{c}\text { Falkland (Malvinas) } \\
\text { Islands }\end{array}$ & Gaevskaya \& Rodyuk (1988) \\
\hline
\end{tabular}




\begin{tabular}{|c|c|c|c|c|c|c|}
\hline Index & Phylum & Family & Species & Host & Location & References \\
\hline 501 & & & Genolinea bowersi & Notothenia corriceps & South Shetland Islands & Zdzitowiecki (1979) \\
\hline 502 & & & Genolinea bowersi & Notothenia coriiceps & Signy Island & Zdzitowiecki et al. (1997) \\
\hline 503 & & & Genolinea bowersi & Notothenia coriiceps & Orkny Islands & Zdzitowiecki et al. (1997) \\
\hline 504 & & & Genolinea bowersi & Notothenia coriiceps & $\begin{array}{l}\text { Potter Cove (King George } \\
\text { Island) }\end{array}$ & Palm et.al. (1998) \\
\hline 505 & & & Genolinea bowersi & Notothenia coriiceps & King George Island & Palm et.al. (1998) \\
\hline 506 & & & Genolinea bowersi & Notothenia coriiceps & Adelie Land & Zdzitowiecki et.al. (1998) \\
\hline 507 & & & Genolinea bowersi & Notothenia coriiceps & Adelie Land & Zdzitowiecki (2001b) \\
\hline 508 & & & Genolinea bowersi & Notothenia coriiceps & Admiralty Bay & $\begin{array}{c}\text { Zdzitowiecki \& Laskowski } \\
\text { (2004) }\end{array}$ \\
\hline 509 & & & Genolinea bowersi & Notothenia coriiceps & Vernadsky Station & $\begin{array}{c}\text { Zdzitowiecki \& Laskowski } \\
\text { (2004) }\end{array}$ \\
\hline 510 & & & Genolinea bowersi & Notothenia cyanobrancha & Kerguelen Subregion & Prudhoe \& Bray (1973) \\
\hline 511 & & & Genolinea bowersi & Notothenia rossii & South Shetland Islands & Zdzitowiecki (1979) \\
\hline 512 & & & Genolinea bowersi & Notothenia rossii & South Georgia Island & Zdzitowiecki (1979) \\
\hline 513 & & & Genolinea bowersi & Notothenia rossii & South Georgia Island & Zdzitowiecki (1991) \\
\hline 514 & & & Genolinea bowersi & Notothenia rossii & South Shetland Islands & Zdzitowiecki (2002b) \\
\hline 515 & \multirow{12}{*}{ DIGENEA } & Hemiuridae & Genolinea bowersi & Parachaenichthys charcoti & Admiralty Bay & Zdzitowiecki (2001a) \\
\hline 516 & & & Genolinea bowersi & Parachaenichthys charcoti & $\begin{array}{l}\text { Off the South Shetland } \\
\text { Islands }\end{array}$ & Zdzitowiecki (2001a) \\
\hline 517 & & & Genolinea bowersi & Parachaenichthys charcoti & Bransfield Strait & Zdzitowiecki (2001a) \\
\hline 518 & & & Genolinea bowersi & Parachaenichthys georgianus & South Georgia Island & Zdzitowiecki (1979) \\
\hline 519 & & & Genolinea bowersi & Parachaenichthys georgianus & South Georgia Island & Zdzitowiecki (1991) \\
\hline 520 & & & Genolinea bowersi & Pleurogramma antarctica & South Shetland Islands & Zdzitowiecki (1979) \\
\hline 521 & & & Genolinea bowersi & Trematomus bernacchii & South Shetland Islands & Zdzitowiecki (1979) \\
\hline 522 & & & Genolinea bowersi & Trematomus bernacchii & Adelie Land & Zdzitowiecki et.al. (1998) \\
\hline 523 & & & Genolinea bowersi & Trematomus bernacchii & Admiralty Bay & $\begin{array}{l}\text { Laskowski \& Zdzitowiecki } \\
\qquad(2005)\end{array}$ \\
\hline 524 & & & Genolinea bowersi & Trematomus bernacchii & Vernadsky Station & $\begin{array}{l}\text { Laskowski \& Zdzitowiecki } \\
\text { (2005) }\end{array}$ \\
\hline 525 & & & Genolinea bowersi & Trematomus hansoni & Adelie Land & Zdzitowiecki et.al. (1998) \\
\hline 526 & & & Genolinea bowersi & Trematomus hansoni & Adelie Land & Zdzitowiecki (2001b) \\
\hline
\end{tabular}




\begin{tabular}{|c|c|c|c|c|c|c|}
\hline Index & Phylum & Family & Species & Host & Location & References \\
\hline 527 & & & Genolinea bowersi & Trematomus lepidorhinus & Weddell Sea & Zdzitowiecki (2002b) \\
\hline 528 & & & Genolinea bowersi & Trematomus loennbergi & Weddell Sea & Zdzitowiecki (2002b) \\
\hline 529 & & & Genolinea bowersi & Trematomus newnesi & $66^{\circ} 45^{\prime} \mathrm{S} 64^{\circ} 52^{\prime} \mathrm{E}$ & Prudhoe \& Bray (1973) \\
\hline 530 & & & Genolinea bowersi & Trematomus newnesi & $\begin{array}{l}\text { Adelie Land (Eastern } \\
\text { Antarctica) }\end{array}$ & Laskowski et.al. (2007) \\
\hline 531 & & & Genolinea bowersi & Trematomus newnesi & Admiralty Bay & $\begin{array}{l}\text { Laskowski \& Zdzitowiecki } \\
\text { (2005) }\end{array}$ \\
\hline 532 & & & Genolinea bowersi & Trematomus newnesi & Vernadsky Station & $\begin{array}{l}\text { Laskowski \& Zdzitowiecki } \\
\text { (2005) }\end{array}$ \\
\hline 533 & & & Genolinea bowersi & Trematomus nicolai & Weddell Sea & Zdzitowiecki (2002b) \\
\hline 534 & & & Genolinea bowersi & Trematomus nicolai & A.A.E. & Prudhoe \& Bray (1973) \\
\hline 535 & & & Genolinea bowersi & Trematomus nicolai & $64^{\circ} 44^{\prime} \mathrm{S} 97^{\circ} 28^{\prime} \mathrm{E}$ & Prudhoe \& Bray (1973) \\
\hline 536 & & & Genolinea bowersi & Trematomus scotti & $67^{\circ} 03^{\prime} \mathrm{S} 74^{\circ} 29^{\prime} \mathrm{E}$ & Prudhoe \& Bray (1973) \\
\hline 537 & & & Genolinea bowersi & Trematomиs pennellii & A.A.E. & Prudhoe \& Bray (1973) \\
\hline 538 & & & Genolinea bowersi & Trematomus pennellii & $65^{\circ} 42^{\prime} \mathrm{S} 92^{\circ} 10^{\prime} \mathrm{E}$ & Prudhoe \& Bray (1973) \\
\hline 539 & & & Genolinea bowersi & HOST NAME NOT MENTIONED & Kerguelen Subregion & Lyadov (1985) \\
\hline 540 & & & Genolinea bowersi & HOST NAME NOT MENTIONED & Glacial Subregion & Lyadov (1985) \\
\hline 541 & & & Glomericirrus macrouri & blank & blank & Gaevskaya (1973) \\
\hline 542 & \multirow{11}{*}{ DIGENEA } & & Glomericirrus macrouri & Cryodraco antarcticus & Weddell Sea & Zdzitowiecki (2002a) \\
\hline 543 & & Hemiuridae & Glomericirrus macrouri & Dissostichus eleginoides & Falkland-Patagonian Shelf & Gaevskaya et al. (1990) \\
\hline 544 & & & Glomericirrus macrouri & Dissostichus eleginoides & Prince Edward Island & Brickle et al. (2005) \\
\hline 545 & & & Glomericirrus macrouri & Dissostichus eleginoides & Heard Island & Brickle et al. (2005) \\
\hline 546 & & & Glomericirrus macrouri & Dissostichus eleginoides & Macquarie Island & Brickle et al. (2005) \\
\hline 547 & & & Glomericirrus macrouri & Dissostichus eleginoides & Ross Sea & Brickle et al. (2005) \\
\hline 548 & & & Glomericirrus macrouri & Dissostichus eleginoides & Falkland Islands & Brickle et al. (2006) \\
\hline 549 & & & Glomericirrus macrouri & Dolloidraco longedorsalis & Weddell Sea & Zdzitowiecki (2002c) \\
\hline 550 & & & Glomericirrus macrouri & Trematomus eulepidotus & Weddell Sea & Zdzitowiecki (2002b) \\
\hline 551 & & & Glomericirrus macrouri & Trematomus lepidorhinus & Weddell Sea & Zdzitowiecki (2002b) \\
\hline 552 & & & Glomericirrus macrouri & Trematomus loennbergi & Weddell Sea & Zdzitowiecki (2002b) \\
\hline
\end{tabular}




\begin{tabular}{|c|c|c|c|c|c|c|}
\hline Index & Phylum & Family & Species & Host & Location & References \\
\hline 553 & & & Glomericirrus macrouri & Macrourus carinatus & $\begin{array}{l}\text { Falkland (Malvinas) } \\
\text { Islands }\end{array}$ & Gaevskaya \& Rodyuk (1988) \\
\hline 554 & & & Glomericirrus macrouri & Macrourus whitsoni & Weddell Sea & Zdzitowiecki \& Cielecka (1997a) \\
\hline 555 & & & Glomericirrus macrouri & Macrourus whitsoni & Weddell Sea & Zdzitowiecki (2003) \\
\hline 556 & & & Glomericirrus macrouri & Parachaenichthys charcoti & Bransfield Strait & Zdzitowiecki (2001a) \\
\hline 557 & & & Glomericirrus macrouri & HOST NAME NOT MENTIONED & Glacial Subregion & Lyadov (1985) \\
\hline 558 & & & Glomericirrus macrouri & HOST NAME NOT MENTIONED & Kerguelen Subregion & Lyadov (1985) \\
\hline 559 & & & Lecithochirium sp. & blank & blank & Lühe (1901) \\
\hline 560 & & & Lecithochirium sp. & Nototheniops larseni & Ob Bank & Parukhin (1986) \\
\hline 561 & & & Lecithochirium sp. & Dissostichus eleginoides & Macquarie Island & Brickle et al. (2005) \\
\hline 562 & & & Lecithochirium sp. & Macrourus holotrachys & North Scotia Ridge & Zdzitowiecki \& Cielecka (1998) \\
\hline 563 & & & Lecithochirium genypteri & blank & blank & Lühe (1905) \\
\hline 564 & & & Lecithochirium genypteri & Genypterus blacodes & South Western Atlantic & Gaevskaya \& Kovaleva (1978) \\
\hline 565 & & & Lecithochirium genypteri & Dissostichus eleginoides & Falkland Islands & Brickle et al. (2006) \\
\hline 566 & & & Lecithochirium genypteri & Dissostichus eleginoides & Falkland-Patagonian Shelf & Gaevskaya et al. (1990) \\
\hline 567 & & & Lecithochirium whitei & blank & blank & Zdzitowiecki (1994) \\
\hline 568 & & & Lecithochirium whitei & Bathylagus antarcticus & South Georgia Island & Zdzitowiecki (1994) \\
\hline 569 & & & Lecithocladium cristatum & blank & blank & Rudolphi (1819) \\
\hline 570 & & & Lecithocladium cristatum & blank & blank & Looss (1907) \\
\hline 571 & & & Lecithocladium cristatum & HOST NAME NOT MENTIONED & Glacial Subregion & Lyadov (1985) \\
\hline 572 & \multirow{8}{*}{ DIGENEA } & Hemiuridae & Lecithocladium falklandicum & blank & blank & Gaevskaya \& Kovaleva (1978) \\
\hline 573 & & & Lecithocladium falklandicum & Stromateus maculatus & South Western Atlantic & Gaevskaya \& Kovaleva (1978) \\
\hline 574 & & & Parahemiurus oatesi & blank & blank & Leiper \& Atkinson (1914) \\
\hline 575 & & & Parahemiurus oatesi & blank & blank & $\begin{array}{l}\text { Skrjabin \& Guschanskaja (1954) } \\
\quad=\text { Elythrophalloides oatesi }\end{array}$ \\
\hline 576 & & & Parahemiurus oatesi & Chaenocephalus aceratus & South Georgia Island & Parukhin \& Lyadov (1981) \\
\hline 577 & & & Parahemiurus oatesi & Champsocephalus gunnari & South Georgia Island & Parukhin \& Lyadov (1981) \\
\hline 578 & & & Parahemiurus oatesi & Dissostichus eleginoides & Shag Rocks & Parukhin \& Lyadov (1981) \\
\hline 579 & & & Parahemiurus oatesi & Gobionotothen gibberifrons & South Georgia Island & Parukhin \& Lyadov (1981) \\
\hline
\end{tabular}




\begin{tabular}{|c|c|c|c|c|c|c|}
\hline Index & Phylum & Family & Species & Host & Location & References \\
\hline 580 & & & Parahemiurus oatesi & Notothenia rossii & South Georgia Island & Parukhin \& Lyadov (1981) \\
\hline 581 & & & Parahemiurus oatesi & Patagonotothen ramsayi & Falkland Islands & Parukhin \& Lyadov (1981) \\
\hline 582 & & & Parahemiurus oatesi & Pseudochaenichthys georgianus & South Georgia Island & Parukhin \& Lyadov (1981) \\
\hline 583 & & Lecithasteridae & Genarches lintoni $=$ Genolinea bowersi & Eleginops maclovinus & South Shetland Islands & Szidat \& Graefe (1967) \\
\hline 584 & & & Genarches lintoni $=$ Genolinea bowersi & Parachaenichthys charcoti & South Shetland Islands & Szidat \& Graefe (1967) \\
\hline 585 & & & Lecithaster sp. & Parachaenichthys charcoti & South Shetland Islands & Lühe (1901) \\
\hline 586 & & & Lecithaster sp. & Chaenocephalus aceratus & South Georgia Island & Parukhin \& Lyadov (1981) \\
\hline 587 & & & Lecithaster australis & Chaenocephalus aceratus & South Georgia Island & Prudhoe \& Bray (1973) \\
\hline 588 & & & Lecithaster australis & Chaenocephalus aceratus & South Shetland Islands & Zdzitowiecki (1979) \\
\hline 589 & & & Lecithaster australis & Chaenocephalus aceratus & South Georgia Island & Zdzitowiecki (1979) \\
\hline 590 & & & Lecithaster australis & Chaenichthys rhinoceratus & Off Murray Island & Prudhoe \& Bray (1973) \\
\hline 591 & & & Lecithaster australis & Chaenichthys rhinoceratus & Kerguelen Subregion & Prudhoe \& Bray (1973) \\
\hline 592 & & & Lecithaster australis & Chaenichthys rhinoceratus & Kerguelen Subregion & Parukhin \& Lyadov (1981) \\
\hline 593 & & & Lecithaster australis & Chaenichthys rhinoceratus & Kerguelen Subregion & Lyadov et al. (1981) \\
\hline 594 & & & Lecithaster australis & Chaenichthys velifer & Kerguelen Subregion & Parukhin \& Lyadov (1981) \\
\hline 595 & & & Lecithaster australis & Chaenichthys velifer & Kerguelen Subregion & Lyadov et al. (1981) \\
\hline 596 & & & Lecithaster australis & Champsocephalus gunnari & $49^{\circ} 28^{\prime} \mathrm{S} 70^{\circ} 33^{\prime} \mathrm{E}$ & Prudhoe \& Bray (1973) \\
\hline 597 & & & Lecithaster australis & Champsocephalus gunnari & $\begin{array}{l}\text { Off Entrance To Royal } \\
\text { Sound (Kerguelen } \\
\text { Subregion) }\end{array}$ & Prudhoe \& Bray (1973) \\
\hline 598 & & & Lecithaster australis & Champsocephalus gunnari & Kerguelen Subregion & Parukhin \& Lyadov (1981) \\
\hline 599 & & & Lecithaster australis & Champsocephalus gunnari & Kerguelen Subregion & Lyadov et al. (1981) \\
\hline 600 & \multirow{6}{*}{ DIGENEA } & Lecithasteridae & Lecithaster australis & Dissostichus eleginoides & Kerguelen Subregion & Parukhin \& Lyadov (1981) \\
\hline 601 & & & Lecithaster australis & Dissostichus eleginoides & Ob Bank & Parukhin \& Lyadov (1981) \\
\hline 602 & & & Lecithaster australis & Dissostichus eleginoides & Crozet Islands & Parukhin \& Lyadov (1981) \\
\hline 603 & & & Lecithaster australis & Dissostichus eleginoides & Kerguelen Subregion & Parukhin \& Lyadov (1982) \\
\hline 604 & & & Lecithaster australis & Dissostichus eleginoides & Skif Bank & Parukhin \& Lyadov (1982) \\
\hline 605 & & & Lecithaster australis & Dissostichus eleginoides & Ob Bank & Parukhin \& Lyadov (1982) \\
\hline
\end{tabular}




\begin{tabular}{|c|c|c|c|c|c|c|}
\hline Index & Phylum & Family & Species & Host & Location & References \\
\hline 606 & & & Lecithaster australis & Dissostichus eleginoides & Falkland-Patagonian Shelf & Gaevskaya et al. (1990) \\
\hline 607 & & & Lecithaster australis & Dissostichus eleginoides & Falkland Islands & Brickle et al. (2006) \\
\hline 608 & & & Lecithaster australis & Gobionotothen acuta & $\begin{array}{l}\text { Near Head of Bras } \\
\text { Bossiere (Kerguelen } \\
\text { Subregion) }\end{array}$ & Prudhoe \& Bray (1973) \\
\hline 609 & & & Lecithaster australis & Harpagifer bispinis & $54^{\circ} 42^{\prime} 30^{\prime \prime} \mathrm{S} 158^{\circ} 54^{\prime} 30^{\prime \prime} \mathrm{E}$ & Prudhoe \& Bray (1973) \\
\hline 610 & & & Lecithaster australis & Harpagifer bispinis & $\begin{array}{l}\text { Off Lusitania Bay, } \\
\text { Macquarie Island }\end{array}$ & Prudhoe \& Bray (1973) \\
\hline 611 & & & Lecithaster australis & Lepidonotothen squamifrons & Kerguelen Subregion & Parukhin \& Lyadov (1981) \\
\hline 612 & & & Lecithaster australis & Lepidonotothen squamifrons & Ob Bank & Parukhin \& Lyadov (1981) \\
\hline 613 & & & Lecithaster australis & Lepidonotothen squamifrons & Lena Bank & Parukhin \& Lyadov (1981) \\
\hline 614 & & & Lecithaster australis & Lepidonotothen squamifrons & Kerguelen Subregion & Parukhin \& Lyadov (1982) \\
\hline 615 & & & Lecithaster australis & Lepidonotothen squamifrons & Skif Bank & Parukhin \& Lyadov (1982) \\
\hline 616 & & & Lecithaster australis & Lepidonotothen squamifrons & Ob Bank & Parukhin \& Lyadov (1982) \\
\hline 617 & & & Lecithaster australis & Lepidonotothen squamifrons & Lena Bank & Parukhin \& Lyadov (1982) \\
\hline 618 & & & Lecithaster australis & Lepidonotothen squamifrons & Lena Bank & Parukhin (1986) \\
\hline 619 & & & Lecithaster australis & Nototheniidae & Kerguelen Subregion & Parukhin \& Lyadov (1982) \\
\hline 620 & & & Lecithaster australis & Nototheniidae & Kerguelen Subregion & Parukhin \& Lyadov (1982) \\
\hline 621 & & & Lecithaster australis & Notothenia colbecki & A.A.E. & Prudhoe \& Bray (1973) \\
\hline 622 & & & Lecithaster australis & Notothenia colbecki & Macquarie Island & Prudhoe \& Bray (1973) \\
\hline 623 & & & Lecithaster australis & Notothenia coriiceps & Heard Island & Prudhoe \& Bray (1973) \\
\hline 624 & & & Lecithaster australis & Notothenia corriceps & South Shetland Islands & Zdzitowiecki (1979) \\
\hline 625 & & & Lecithaster australis & Notothenia corriceps & South Georgia Island & Zdzitowiecki (1979) \\
\hline 626 & & & Lecithaster australis & Notothenia cyanobrancha & Royal Sound & Prudhoe \& Bray (1973) \\
\hline 627 & & & Lecithaster australis & Notothenia cyanobrancha & Kerguelen Subregion & Prudhoe \& Bray (1973) \\
\hline 628 & \multirow{4}{*}{ DIGENEA } & Lecithasteridae & Lecithaster australis & Notothenia macrocephala & A.A.E. & Prudhoe \& Bray (1973) \\
\hline 629 & & & Lecithaster australis & Notothenia macrocephala & Macquarie Island & Prudhoe \& Bray (1973) \\
\hline 630 & & & Lecithaster australis & Notothenia magellanica & Ob Bank & Parukhin (1986) \\
\hline 631 & & & Lecithaster australis & Notothenia ramsayi & South Western Atlantic & Gaevskaya \& Kovaleva (1978) \\
\hline
\end{tabular}




\begin{tabular}{|c|c|c|c|c|c|c|}
\hline Index & Phylum & Family & Species & Host & Location & References \\
\hline 632 & & & Lecithaster australis & Notothenia rossii & A.A.E. & Prudhoe \& Bray (1973) \\
\hline 633 & & & Lecithaster australis & Notothenia rossii & Macquarie Island & Prudhoe \& Bray (1973) \\
\hline 634 & & & Lecithaster australis & Notothenia rossii & South Shetland Islands & Zdzitowiecki (1979) \\
\hline 635 & & & Lecithaster australis & Notothenia rossii & South Georgia Island & Zdzitowiecki (1980) \\
\hline 636 & & & Lecithaster australis & Notothenia rossii & Kerguelen Subregion & Parukhin \& Lyadov (1981) \\
\hline 637 & & & Lecithaster australis & Notothenia rossii & Heard Island & Parukhin \& Lyadov (1982) \\
\hline 638 & & & Lecithaster australis & Notothenia rossii & Crozet Islands & Parukhin \& Lyadov (1983) \\
\hline 639 & & & Lecithaster australis & Notothenia rossii & Skif Bank & Parukhin \& Lyadov (1984) \\
\hline 640 & & & Lecithaster australis & Notothenia rossii & Ob Bank & Parukhin \& Lyadov (1985) \\
\hline 641 & & & Lecithaster australis & Notothenia rossii & Kerguelen Subregion & Parukhin \& Lyadov (1982) \\
\hline 642 & & & Lecithaster australis & Notothenia rossii & Heard Island & Parukhin \& Lyadov (1983) \\
\hline 643 & & & Lecithaster australis & Notothenia rossii & Crozet Islands & Parukhin \& Lyadov (1984) \\
\hline 644 & & & Lecithaster australis & Notothenia rossii & Skif Bank & Parukhin \& Lyadov (1985) \\
\hline 645 & & & Lecithaster australis & Notothenia rossii & Lena Bank & Parukhin (1986) \\
\hline 646 & & & Lecithaster australis & HOST NAME NOT MENTIONED & Glacial Subregion & Lyadov (1985) \\
\hline 647 & & & Lecithaster australis & HOST NAME NOT MENTIONED & Kerguelen Subregion & Lyadov (1986) \\
\hline 648 & & & Lecithaster macrocotyle & blank & blank & Szidat \& Graefe (1967) \\
\hline 649 & & & Lecithaster macrocotyle & Chaenocephalus aceratus & South Georgia Island & Zdzitowiecki (1991) \\
\hline 650 & & & Lecithaster macrocotyle & Chaenocephalus aceratus & $\begin{array}{l}\text { South Shetland Islands } \\
\text { (Admiralty Bay, } \\
\text { Continental Shelves, off } \\
\text { Elephant Island, Loinville } \\
\text { Island) }\end{array}$ & Zdzitowiecki (1992) \\
\hline 651 & & & Lecithaster macrocotyle & Chaenocephalus aceratus & Signy Island & Zdzitowiecki et al. (1997) \\
\hline 652 & & & Lecithaster macrocotyle & Chaenocephalus aceratus & Orkny Islands & Zdzitowiecki et al. (1997) \\
\hline 653 & & & Lecithaster macrocotyle & Chaenocephalus aceratus & South Shetland Islands & Palm et al. (2007) \\
\hline 654 & & & Lecithaster macrocotyle & Chiondraco rastrospinosus & $\begin{array}{l}\text { South Shetland Islands } \\
\text { (Admiralty Bay, } \\
\text { Continental Shelves, off } \\
\text { Elephant Island, Loinville } \\
\text { Island) }\end{array}$ & Zdzitowiecki (1992) \\
\hline
\end{tabular}




\begin{tabular}{|c|c|c|c|c|c|c|}
\hline Index & Phylum & Family & Species & Host & Location & References \\
\hline 655 & & Lecithasteridae & Lecithaster macrocotyle & Chionodraco rastrospinosus & South Shetland Islands & Zdzitowiecki (1991) \\
\hline 656 & & & Lecithaster macrocotyle & Chionodraco rastrospinosus & South Shetland Islands & Zdzitowiecki (2002a) \\
\hline 657 & & & Lecithaster macrocotyle & Cryodraco antarcticus & South Shetland Islands & Zdzitowiecki (1991) \\
\hline 658 & & & Lecithaster macrocotyle & Cryodraco antarcticus & $\begin{array}{c}\text { South Shetland Islands } \\
\text { (Admiralty Bay, } \\
\text { Continental Shelves, off } \\
\text { Elephant Island, Loinville } \\
\text { Island) }\end{array}$ & Zdzitowiecki (1992) \\
\hline 659 & & & Lecithaster macrocotyle & Cryodraco antarcticus & Admiralty Bay & Zdzitowiecki (2002a) \\
\hline 660 & & & Lecithaster macrocotyle & Dissostichus eleginoides & Shag Rocks & Brickle et al. (2005) \\
\hline 661 & & & Lecithaster macrocotyle & Dissostichus eleginoides & Heard Island & Brickle et al. (2005) \\
\hline 662 & & & Lecithaster macrocotyle & Dissostichus mawsoni & South Shetland Islands & Zdzitowiecki (1991) \\
\hline 663 & & & Lecithaster macrocotyle & Dissostichus mawsoni & $\begin{array}{l}\text { South Shetland Islands } \\
\text { (Admiralty Bay, } \\
\text { Continental Shelves, off } \\
\text { Elephant Island, Loinville } \\
\text { Island) }\end{array}$ & Zdzitowiecki (1992) \\
\hline 664 & DIGENEA & & Lecithaster macrocotyle & Lepidonotothen squamifrons & South Georgia Island & Zdzitowiecki (1991) \\
\hline 665 & & & Lecithaster macrocotyle & Lindbergichthys nudifrons & South Georgia Island & Zdzitowiecki (1991) \\
\hline 666 & & & Lecithaster macrocotyle & Lindbergichthys nudifrons & $\begin{array}{l}\text { South Shetland Islands } \\
\text { (Admiralty Bay, } \\
\text { Continental Shelves, off } \\
\text { Elephant Island, Loinville } \\
\text { Island) }\end{array}$ & Zdzitowiecki (1992) \\
\hline 667 & & & Lecithaster macrocotyle & Lindbergichthys nudifrons & $\begin{array}{l}\text { Environs of South } \\
\text { Geoegia (Cumberland } \\
\text { Bay, Stromness Harbour, } \\
\text { Continental Shelves of } \\
\text { South Georgia) }\end{array}$ & Zdzitowiecki (1992) \\
\hline 668 & & & Lecithaster macrocotyle & Lindbergichthys nudifrons & South Shetland Islands & Palm et al. (2007) \\
\hline 669 & & & Lecithaster macrocotyle & Muraenolepis microps & South Georgia Island & Zdzitowiecki (1991) \\
\hline 670 & & & Lecithaster macrocotyle & Notothenia coriiceps & $\begin{array}{l}\text { South Shetland Islands } \\
\text { (Admiralty Bay, } \\
\text { Continental Shelves, off } \\
\text { Elephant Island, Loinville } \\
\text { Island) }\end{array}$ & Zdzitowiecki (1992) \\
\hline 671 & & & Lecithaster macrocotyle & Notothenia coriiceps & $\begin{array}{l}\text { Environs of South } \\
\text { Geoegia (Cumberland } \\
\text { Bay, Stromness Harbour, }\end{array}$ & Zdzitowiecki (1992) \\
\hline
\end{tabular}




\begin{tabular}{|c|c|c|c|c|c|c|}
\hline Index & Phylum & Family & Species & Host & Location & References \\
\hline & & & & & $\begin{array}{l}\text { Continental Shelves of } \\
\text { South Georgia) }\end{array}$ & \\
\hline 672 & & Lecithasteridae & Lecithaster macrocotyle & Notothenia coriiceps & Admiralty Bay & $\begin{array}{c}\text { Zdzitowiecki \& Laskowski } \\
\text { (2004) }\end{array}$ \\
\hline 673 & & & Lecithaster macrocotyle & Notothenia coriiceps & Vernadsky Station & $\begin{array}{c}\text { Zdzitowiecki \& Laskowski } \\
\text { (2004) }\end{array}$ \\
\hline 674 & & & Lecithaster macrocotyle & Notothenia rossii & South Georgia Island & Zdzitowiecki (1991) \\
\hline 675 & & & Lecithaster macrocotyle & Notothenia rossii & $\begin{array}{l}\text { South Shetland Islands } \\
\text { (Admiralty Bay, } \\
\text { Continental Shelves, off } \\
\text { Elephant Island, Loinville } \\
\text { Island) }\end{array}$ & Zdzitowiecki (1992) \\
\hline 676 & & & Lecithaster macrocotyle & Notothenia rossii & $\begin{array}{c}\text { Environs of South } \\
\text { Geoegia (Cumberland } \\
\text { Bay, Stromness Harbour, } \\
\text { Continental Shelves of } \\
\text { South Georgia) }\end{array}$ & \\
\hline 677 & & & Lecithaster macrocotyle & Nototheniops larseni & South Shetland Islands & Palm et al. (2007) \\
\hline 678 & & & Lecithaster macrocotyle & Nototheniops nybelini & South Shetland Islands & Zdzitowiecki (1991) \\
\hline 679 & DIGENEA & & Lecithaster macrocotyle & Nototheniops nybelini & $\begin{array}{l}\text { South Shetland Islands } \\
\text { (Admiralty Bay, } \\
\text { Continental Shelves, off } \\
\text { Elephant Island, Loinville } \\
\text { Island) }\end{array}$ & Zdzitowiecki (1992) \\
\hline 680 & & & Lecithaster macrocotyle & Pagothenia hansoni & $\begin{array}{l}\text { South Shetland Islands } \\
\text { (Admiralty Bay, } \\
\text { Continental Shelves, off } \\
\text { Elephant Island, Loinville } \\
\text { Island) }\end{array}$ & Zdzitowiecki (1992) \\
\hline 681 & & & Lecithaster macrocotyle & Parachaenichthys charcoti & South Shetland Islands & Szidat \& Graefe (1967) \\
\hline 682 & & & Lecithaster macrocotyle & Parachaenichthys charcoti & South Shetland Islands & Zdzitowiecki (1991) \\
\hline 683 & & & Lecithaster macrocotyle & Parachaenichthys charcoti & $\begin{array}{l}\text { South Shetland Islands } \\
\text { (Admiralty Bay, } \\
\text { Continental Shelves, off } \\
\text { Elephant Island, Loinville } \\
\text { Island) }\end{array}$ & Zdzitowiecki (1992) \\
\hline 684 & & & Lecithaster macrocotyle & Parachaenichthys charcoti & Admiralty Bay & Zdzitowiecki (2001a) \\
\hline 685 & & & Lecithaster macrocotyle & Parachaenichthys charcoti & $\begin{array}{l}\text { Off the South Shetland } \\
\text { Islands }\end{array}$ & Zdzitowiecki (2001a) \\
\hline 686 & & & Lecithaster macrocotyle & Parachaenichthys charcoti & Bransfield Strait & Zdzitowiecki (2001a) \\
\hline 687 & & & Lecithaster macrocotyle & Parachaenichthys charcoti & Admiralty Bay & Zdzitowiecki (2001a) \\
\hline
\end{tabular}




\begin{tabular}{|c|c|c|c|c|c|c|}
\hline Index & Phylum & Family & Species & Host & Location & References \\
\hline 688 & & & Lecithaster macrocotyle & Parachaenichthys charcoti & South Shetland Islands & Palm et al. (2007) \\
\hline 689 & & & Lecithaster macrocotyle & Parachaenichthys georgianus & South Georgia Island & Zdzitowiecki (1991) \\
\hline 690 & & & Lecithaster macrocotyle & Parachaenichthys georgianus & $\begin{array}{l}\text { Environs of South } \\
\text { Geoegia (Cumberland } \\
\text { Bay, Stromness Harbour, } \\
\text { Continental Shelves of } \\
\text { South Georgia) }\end{array}$ & Zdzitowiecki (1992) \\
\hline 691 & \multirow{20}{*}{ DIGENEA } & Lecithasteridae & Lecithaster macrocotyle & Patagonotothen brevicauda guntheri & South Georgia Island & Zdzitowiecki (1991) \\
\hline 692 & & & Lecithaster macrocotyle & Pseudochaenichthys georgianus & South Georgia Island & Zdzitowiecki (1991) \\
\hline 693 & & & Lecithaster macrocotyle & Psilodraco breviceps & South Georgia Island & Zdzitowiecki (1991) \\
\hline 694 & & & Lecithaster macrocotyle & Psilodraco breviceps & $\begin{array}{l}\text { Environs of South } \\
\text { Geoegia (Cumberland } \\
\text { Bay, Stromness Harbour, } \\
\text { Continental Shelves of } \\
\text { South Georgia) }\end{array}$ & Zdzitowiecki (1992) \\
\hline 695 & & & Lecithaster macrocotyle & HOST NAME NOT MENTIONED & Subantarctic Region & Zdzitowiecki (1990b) \\
\hline 696 & & & Lecithaster micropsi & Muraenolepis microps & South Georgia Island & Zdzitowiecki (1992) \\
\hline 697 & & & Lecithaster micropsi & Lepidonotothen squamifrons & Shag Rocks & Zdzitowiecki (1993) \\
\hline 698 & & & Lecithaster micropsi & Patagonotothen brevicauda guntheri & Shag Rocks & Zdzitowiecki (1994) \\
\hline 699 & & & Lecithaster micropsi & Chaenocephalus aceratus & Shag Rocks & Zdzitowiecki (1995) \\
\hline 700 & & & Lecithaster micropsi & Pseudochaenichthys georgianus & Shag Rocks & Zdzitowiecki (1996) \\
\hline 701 & & & Lecithaster micropsi & Parachaenichthys georgianus & Shag Rocks & Zdzitowiecki (1997) \\
\hline 702 & & & Lecithophyllum anteroporum & blank & blank & Margolis (1958) \\
\hline 703 & & & Lecithophyllum anteroporum & Lepidonotothen squamifrons & Kerguelen Subregion & Parukhin \& Lyadov (1982) \\
\hline 704 & & & Lecithophyllum anteroporum & Lepidonotothen squamifrons & Skif Bank & Parukhin \& Lyadov (1982) \\
\hline 705 & & & Lecithophyllum anteroporum & Lepidonotothen squamifrons & Ob Bank & Parukhin (1986) \\
\hline 706 & & & Lecithophyllum anteroporum & Notothenia rossii & Ob Bank & Parukhin (1986) \\
\hline 707 & & & Lecithophyllum anteroporum & HOST NAME NOT MENTIONED & Glacial Subregion & Lyadov (1985) \\
\hline 708 & & & Lecithophyllum anteroporum & HOST NAME NOT MENTIONED & Kerguelen Subregion & Lyadov (1985) \\
\hline 709 & & & Lecithophyllum anteroporum & Nototheniidae & Kerguelen Subregion & Parukhin \& Lyadov (1982) \\
\hline 710 & & & Lecithophyllum anteroporum & Lepidonotothen squamifrons & Kerguelen Subregion & Parukhin \& Lyadov (1981) \\
\hline
\end{tabular}




\begin{tabular}{|c|c|c|c|c|c|c|}
\hline Index & Phylum & Family & Species & Host & Location & References \\
\hline 711 & & & Lecithophyllum anteroporum & Lepidonotothen squamifrons & Skif Bank & Parukhin \& Lyadov (1981) \\
\hline 712 & & & Lecithophyllum champsocephali & blank & blank & Zdzitowiecki (1989) \\
\hline 713 & & & Lecithophyllum champsocephali & Champsocephalus gunnari & South Shetland Islands & Zdzitowiecki (2002a) \\
\hline 714 & & & Lecithophyllum champsocephali & Dissostichus eleginoides & Prince Edward Island & Brickle et al. (2005) \\
\hline 715 & & & Lecithophyllum champsocephali & Dissostichus eleginoides & Heard Island & Brickle et al. (2005) \\
\hline 716 & & & Lecithophyllum champsocephali & Dissostichus eleginoides & Macquarie Island & Brickle et al. (2005) \\
\hline 717 & & Lepocreadiidae & Gibsonia hastata & blank & blank & Gaevskaya \& Rodjuk (1988) \\
\hline 718 & & & Gibsonia hastata & Macrourus carinatus & $\begin{array}{l}\text { Falkland (Malvinas) } \\
\text { Islands }\end{array}$ & Gaevskaya \& Rodyuk (1988) \\
\hline 719 & & & Gibsonia hastata & Macrourus holotrachys & North Scotia Ridge & Zdzitowiecki \& Cielecka (1998) \\
\hline 720 & & & Lepidapedon sp. & blank & blank & Stafford (1904) \\
\hline 721 & & & Lepidapedon sp. & Patagonotothen ramsayi & South Western Atlantic & Gaevskaya \& Kovaleva (1978) \\
\hline 722 & & & Lepidapedon sp. & HOST NAME NOT MENTIONED & Glacial Subregion & Lyadov (1985) \\
\hline 723 & & & Lepidapedon antarcticus & blank & blank & $\begin{array}{c}\text { Byrd }(1963)=\text { L. Garrardi; Leiper } \\
\text { et Atkinson (1914) }\end{array}$ \\
\hline 724 & & & Lepidapedon antarcticus & Artedidraco skottsbergi & $65^{\circ} 48^{\prime} \mathrm{S} 53^{\circ} 16^{\prime} \mathrm{E}$ & Prudhoe \& Bray (1973) \\
\hline 725 & & & Lepidapedon antarcticus & Artedidraco skottsbergi & $66^{\circ} 45^{\prime} \mathrm{S} 62^{\circ} 03^{\prime} \mathrm{E}$ & Prudhoe \& Bray (1973) \\
\hline 726 & & & Lepidapedon antarcticus & Artedidraco shackletoni & A.A.E. & Prudhoe \& Bray (1973) \\
\hline 727 & DIGENEA & & Lepidapedon antarcticus & Artedidraco shackletoni & $65^{\circ} 42^{\prime} \mathrm{S} 92^{\circ} 10^{\prime} \mathrm{E}$ & Prudhoe \& Bray (1973) \\
\hline 728 & & & Lepidapedon antarcticus & Chaenocephalus aceratus & South Shetland Islands & Zdzitowiecki (1979) \\
\hline 729 & & & Lepidapedon antarcticus & Chionodraco kathleenae & A.A.E. & Prudhoe \& Bray (1973) \\
\hline 730 & & & Lepidapedon antarcticus & Chionodraco kathleenae & $65^{\circ} 6^{\prime} \mathrm{S} 96^{\circ} 14^{\prime} \mathrm{E}$ & Prudhoe \& Bray (1973) \\
\hline 731 & & & Lepidapedon antarcticus & Gobionotothen gibberifrons & South Shetland Islands & Zdzitowiecki (1979) \\
\hline 732 & & & Lepidapedon antarcticus & Notothenia corriceps & South Shetland Islands & Zdzitowiecki (1979) \\
\hline 733 & & & Lepidapedon antarcticus & Notothenia rossii & South Shetland Islands & Zdzitowiecki (1979) \\
\hline 734 & & & Lepidapedon antarcticus & Notothenia rossii & South Georgia Island & Zdzitowiecki (1979) \\
\hline 735 & & & Lepidapedon antarcticus & Prionodraco evansii & $66^{\circ} 45^{\prime} \mathrm{S} 62^{\circ} 03^{\prime} \mathrm{E}$ & Prudhoe \& Bray (1973) \\
\hline 736 & & & Lepidapedon antarcticus & Trematomus pennellii & A.A.E. & Prudhoe \& Bray (1973) \\
\hline 737 & & & Lepidapedon antarcticus & Trematomus pennellii & $65^{\circ} 42^{\prime} \mathrm{S} 92^{\circ} 10^{\prime} \mathrm{E}$ & Prudhoe \& Bray (1973) \\
\hline
\end{tabular}




\begin{tabular}{|c|c|c|c|c|c|c|}
\hline Index & Phylum & Family & Species & Host & Location & References \\
\hline 738 & & & Lepidapedon antarcticus & HOST NAME NOT MENTIONED & Glacial Subregion & Lyadov (1985) \\
\hline 739 & & & Lepidapedon balgueriasi & blank & & Zdzitowiecki \& Cielecka (1997) \\
\hline 740 & & & Lepidapedon balgueriasi & Notothenia coriiceps, & Adelie Land & Zdzitowiecki et.al. (1998) \\
\hline 741 & & & Lepidapedon balgueriasi & Trematomus hansoni & Adelie Land & Zdzitowiecki et.al. (1998) \\
\hline 742 & & & Lepidapedon balgueriasi & Trematomus hansoni & Adelie Land & Zdzitowiecki (2001b) \\
\hline 743 & & & Lepidapedon balgueriasi & Trematomus lepidorhinus & Weddell Sea & Zdzitowiecki \& Cielecka (1997c) \\
\hline 744 & & & Lepidapedon balgueriasi & Trematomus lepidorhinus & Weddell Sea & Zdzitowiecki (2002b) \\
\hline 745 & & & Lepidapedon balgueriasi & Trematomus scotti & Weddell Sea & Zdzitowiecki \& Cielecka (1997c) \\
\hline 746 & & Lepocreadiidae & Lepidapedon balgueriasi & Trematomus scotti & Weddell Sea & Zdzitowiecki (2002b) \\
\hline 747 & & & Lepidapedon balgueriasi & Trematomus pennellii & Weddell Sea & Zdzitowiecki \& Cielecka (1997c) \\
\hline 748 & & & Lepidapedon balgueriasi & Trematomus pennellii & Weddell Sea & Zdzitowiecki (2002b) \\
\hline 749 & & & Lepidapedon balgueriasi & Trematomus loennbergi & Weddell Sea & Zdzitowiecki \& Cielecka (1997c) \\
\hline 750 & & & Lepidapedon balgueriasi & Trematomus loennbergi & Weddell Sea & Zdzitowiecki (2002b) \\
\hline 751 & & & Lepidapedon brayi & blank & blank & Zdzitowiecki \& Cielecka (1997) \\
\hline 752 & & & Lepidapedon brayi & Macrourus whitsoni & Weddell Sea & Zdzitowiecki \& Cielecka (1997a) \\
\hline 753 & & & Lepidapedon brayi & Macrourus whitsoni & Weddell Sea & Zdzitowiecki (2003) \\
\hline 754 & & & Lepidapedon garrardi & blank & blank & Leiper \& Atkinson (1914) \\
\hline 755 & DIGENEA & & Lepidapedon garrardi & Artedidraconidae & Weddell Sea & Zdzitowiecki (2002c) \\
\hline 756 & & & Lepidapedon garrardi & Artedidraco loennbergi & Weddell Sea & Zdzitowiecki \& Cielecka (1997c) \\
\hline 757 & & & Lepidapedon garrardi & Artedidraco loennbergi & Weddell Sea & Zdzitowiecki (2002c) \\
\hline 758 & & & Lepidapedon garrardi & Bathydraconidae & Weddell Sea & Zdzitowiecki (2002d) \\
\hline 759 & & & Lepidapedon garrardi & Bathydraco marri & Weddell Sea & Zdzitowiecki \& Cielecka (1997c) \\
\hline 760 & & & Lepidapedon garrardi & Bathydraco marri & Weddell Sea & Zdzitowiecki (2002d) \\
\hline 761 & & & Lepidapedon garrardi & Chionodraco rastrospinosus & South Shetland Islands & Zdzitowiecki (1991) \\
\hline 762 & & & Lepidapedon garrardi & Cryodraco antarcticus & $65^{\circ} 42^{\prime} \mathrm{S} 92^{\circ} 10^{\prime} \mathrm{E}$ & Prudhoe \& Bray (1973) \\
\hline 763 & & & Lepidapedon garrardi & Dissostichus eleginoides & South Shetland Islands & Zdzitowiecki (1991) \\
\hline 764 & & & Lepidapedon garrardi & Gobionotothen gibberifrons & South Shetland Islands & Zdzitowiecki (1991) \\
\hline
\end{tabular}




\begin{tabular}{|c|c|c|c|c|c|c|}
\hline Index & Phylum & Family & Species & Host & Location & References \\
\hline 765 & & & Lepidapedon garrardi & Gobionotothen gibberifrons & South Georgia Island & Zdzitowiecki (1991) \\
\hline 766 & & & Lepidapedon garrardi & Gobionotothen gibberifrons & Signy Island & Zdzitowiecki et al. (1997) \\
\hline 767 & & & Lepidapedon garrardi & Gobionotothen gibberifrons & South Orkney Islands & Zdzitowiecki et al. (1997) \\
\hline 768 & & & Lepidapedon garrardi & Lepidonotothen larseni & $\begin{array}{l}\text { Port Foster (Deception } \\
\text { Island) }\end{array}$ & Ruhl et al. (2003) \\
\hline 769 & & & Lepidapedon garrardi & Lindbergichthys nudifrons & South Shetland Islands & Zdzitowiecki (2002b) \\
\hline 770 & & & Lepidapedon garrardi & Lindbergichthys nudifrons & Vernadsky Station & $\begin{array}{l}\text { Laskowski \& Zdzitowiecki } \\
\text { (2005) }\end{array}$ \\
\hline 771 & & & Lepidapedon garrardi & Lindbergichthys nudifrons & Admiralty Bay & $\begin{array}{l}\text { Laskowski \& Zdzitowiecki } \\
\qquad(2005)\end{array}$ \\
\hline 772 & & & Lepidapedon garrardi & Notothenia coriiceps & $\begin{array}{c}\text { Potter Cove (King George } \\
\text { Island) }\end{array}$ & Palm et.al. (1998) \\
\hline 773 & & & Lepidapedon garrardi & Notothenia coriiceps & King George Island & Palm et.al. (1998) \\
\hline 774 & & & Lepidapedon garrardi & Notothenia coriiceps & Adelie Land & Zdzitowiecki et.al. (1998) \\
\hline 775 & \multirow{16}{*}{ DIGENEA } & Lepocreadiidae & Lepidapedon garrardi & Notothenia coriiceps & Adelie Land & Zdzitowiecki (2001b) \\
\hline 776 & & & Lepidapedon garrardi & Notothenia coriiceps & Admiralty Bay & $\begin{array}{c}\text { Zdzitowiecki \& Laskowski } \\
(2004)\end{array}$ \\
\hline 777 & & & Lepidapedon garrardi & Notothenia coriiceps & Vernadsky Station & $\begin{array}{c}\text { Zdzitowiecki \& Laskowski } \\
\text { (2004) }\end{array}$ \\
\hline 778 & & & Lepidapedon garrardi & Notothenia rossii & South Georgia Island & Zdzitowiecki (1991) \\
\hline 779 & & & Lepidapedon garrardi & Nototheniops larseni & South Georgia Island & Zdzitowiecki (1991) \\
\hline 780 & & & Lepidapedon garrardi & Parachaenichthys charcoti & South Shetland Islands & Zdzitowiecki (1991) \\
\hline 781 & & & Lepidapedon garrardi & Parachaenichthys charcoti & Admiralty Bay & Zdzitowiecki (2001a) \\
\hline 782 & & & Lepidapedon garrardi & Parachaenichthys charcoti & $\begin{array}{l}\text { Off the South Shetland } \\
\text { Islands }\end{array}$ & Zdzitowiecki (2001a) \\
\hline 783 & & & Lepidapedon garrardi & Parachaenichthys charcoti & Bransfield Strait & Zdzitowiecki (2001a) \\
\hline 784 & & & Lepidapedon garrardi & Patagonotothen brevicauda guntheri & South Georgia Island & Zdzitowiecki (1991) \\
\hline 785 & & & Lepidapedon garrardi & Prionodraco evansii & Weddell Sea & Zdzitowiecki \& Cielecka (1997c) \\
\hline 786 & & & Lepidapedon garrardi & Prionodraco evansii & Weddell Sea & Zdzitowiecki (2002d) \\
\hline 787 & & & Lepidapedon garrardi & Trematomus bernacchii & $67^{\circ} \mathrm{S} 142^{\circ} 36^{\prime} \mathrm{E}$ & Prudhoe \& Bray (1973) \\
\hline 788 & & & Lepidapedon garrardi & Trematomus bernacchii & Mcmurdo Sound & Moser \& Cowen (1991) \\
\hline 789 & & & Lepidapedon garrardi & Trematomus bernacchii & Vernadsky Station & $\begin{array}{c}\text { Laskowski \& Zdzitowiecki } \\
\text { (2005) }\end{array}$ \\
\hline 790 & & & Lepidapedon garrardi & Trematomus bernacchii & Admiralty Bay & $\begin{array}{l}\text { Laskowski \& Zdzitowiecki } \\
\text { (2005) }\end{array}$ \\
\hline
\end{tabular}




\begin{tabular}{|c|c|c|c|c|c|c|}
\hline Index & Phylum & Family & Species & Host & Location & References \\
\hline 791 & & & Lepidapedon garrardi & Trematomus eulepidotus & Weddell Sea & Zdzitowiecki \& Cielecka (1997c) \\
\hline 792 & & & Lepidapedon garrardi & Trematomus eulepidotus & Weddell Sea & Zdzitowiecki (2002b) \\
\hline 793 & & & Lepidapedon garrardi & Trematomus hansoni & Weddell Sea & Zdzitowiecki \& Cielecka (1997c) \\
\hline 794 & & & Lepidapedon garrardi & Trematomus hansoni & Weddell Sea & Zdzitowiecki (2002b) \\
\hline 795 & & & Lepidapedon garrardi & Trematomus hansoni & Adelie Land & Zdzitowiecki et.al. (1998) \\
\hline 796 & & & Lepidapedon garrardi & Trematomus hansoni & Adelie Land & Zdzitowiecki (2001b) \\
\hline 797 & & & Lepidapedon garrardi & Trematomus lepidorhinus & Weddell Sea & Zdzitowiecki \& Cielecka (1997c) \\
\hline 798 & & & Lepidapedon garrardi & Trematomus lepidorhinus & Weddell Sea & Zdzitowiecki (2002b) \\
\hline 799 & & & Lepidapedon garrardi & Trematomus loennbergi & Weddell Sea & Zdzitowiecki \& Cielecka (1997c) \\
\hline 800 & & & Lepidapedon garrardi & Trematomus loennbergi & Weddell Sea & Zdzitowiecki (2002b) \\
\hline 801 & & & Lepidapedon garrardi & Trematomus nicolai & Weddell Sea & Zdzitowiecki \& Cielecka (1997c) \\
\hline 802 & & & Lepidapedon garrardi & Trematomus nicolai & Weddell Sea & Zdzitowiecki (2002b) \\
\hline 803 & & Lepocreadiidae & Lepidapedon garrardi & Trematomus scotti & Weddell Sea & Zdzitowiecki \& Cielecka (1997c) \\
\hline 804 & & & Lepidapedon garrardi & Trematomus scotti & Weddell Sea & Zdzitowiecki (2002b) \\
\hline 805 & & & Lepidapedon garrardi & HOST NAME NOT MENTIONED & Glacial Subregion & Lyadov (1985) \\
\hline 806 & & & Lepidapedon lebouri & blank & blank & Manter (1934) \\
\hline 807 & & & Lepidapedon lebouri & $\begin{array}{c}\text { Coryphaenoides = Macrourus } \\
\text { holotrachys }\end{array}$ & South Western Atlantic & Gaevskaya \& Kovaleva (1978) \\
\hline 808 & & & Lepidapedon lepidum & blank & blank & Gaevskaya \& Rodjuk (1988) \\
\hline 809 & & & Lepidapedon lepidum & Macrourus carinatus & $\begin{array}{l}\text { Falkland (Malvinas) } \\
\text { Islands }\end{array}$ & Gaevskaya \& Rodyuk (1988) \\
\hline 810 & DIGENEA & & Lepidapedon ninae & blank & blank & Zdzitowiecki \& Cielecka (1997) \\
\hline 811 & & & Lepidapedon ninae & Macrourus whitsoni & Weddell Sea & Zdzitowiecki \& Cielecka (1997a) \\
\hline 812 & & & Lepidapedon ninae & Macrourus whitsoni & Weddell Sea & Zdzitowiecki (2003) \\
\hline 813 & & & Lepidapedon notogeorgianus & blank & blank & Zdzitowiecki (1990) \\
\hline 814 & & & Lepidapedon notogeorgianus & Dissostichus eleginoides & South Georgia Island & Zdzitowiecki (1991) \\
\hline 815 & & & Lepidapedon notogeorgianus & Lepidonotothen squamifrons & South Georgia Island & Zdzitowiecki (1990a) \\
\hline 816 & & & Lepidapedon notogeorgianus & Lepidonotothen squamifrons & South Georgia Island & Zdzitowiecki (1991) \\
\hline 817 & & & Lepidapedon notogeorgianus & Lindbergichthys nudifrons & South Georgia Island & Zdzitowiecki (1991) \\
\hline
\end{tabular}




\begin{tabular}{|c|c|c|c|c|c|c|}
\hline Index & Phylum & Family & Species & Host & Location & References \\
\hline 818 & & & Lepidapedon notogeorgianus & Patagonothenia brevicauda guntheri & South Georgia Island & Zdzitowiecki (1991) \\
\hline 819 & & & Lepidapedon notogeorgianus & Patagonotothen brevicauda guntheri & Shag Rocks & Zdzitowiecki (1990a) \\
\hline 820 & & & Lepidapedon paralebouri & blank & blank & Zdzitowiecki (1990) \\
\hline 821 & & & Lepidapedon paralebouri & Muraenolepis microps & South Georgia Island & Zdzitowiecki (1990a) \\
\hline 822 & & & Lepidapedon paralebouri & Muraenolepis microps & South Georgia Island & Zdzitowiecki (1991) \\
\hline 823 & & & Lepidapedon taeniatum & blank & blank & Gaevskaya \& Rodyuk (1988) \\
\hline 824 & & & Lepidapedon taeniatum & Dissostichus eleginoides & Falkland-Patagonian Shelf & Gaevskaya et al. (1990) \\
\hline 825 & & & Lepidapedon taeniatum & Macrourus carinatus & $\begin{array}{l}\text { Falkland (Malvinas) } \\
\text { Islands }\end{array}$ & Gaevskaya \& Rodyuk (1988) \\
\hline 826 & & & Lepidapedon tertius & blank & blank & Zdzitowiecki (1990) \\
\hline 827 & & & Lepidapedon tertius & Ophthalmolycus concolor & South Shetland Islands & Zdzitowiecki (1991) \\
\hline 828 & & & Lepidapedon tertius & Ophthalmolycus concolor & South Shetland Islands & Zdzitowiecki (1990a) \\
\hline 829 & & & Lepidapedon tertius & Ophthalmolycus concolor & Shelf off Elephant Island & Zdzitowiecki (1990a) \\
\hline 830 & & & Lepocreadium trullaeforme & blank & blank & $\begin{array}{c}\text { Linton }(1940)=\text { Lepidapedon } \\
\text { garrardi }\end{array}$ \\
\hline 831 & & & Lepocreadium trullaeforme & Nototheniidae & Kerguelen Subregion & Parukhin \& Lyadov (1982) \\
\hline 832 & & Lepocreadiidae & Lepocreadium trullaeforme & Notothenia coriiceps & $\begin{array}{c}\text { Bhaia Scotia La Isla } \\
\text { Laurie (Orcadas del sur, } \\
\text { Argentina) }\end{array}$ & Szidat (1965) \\
\hline 833 & & & Lepocreadium trullaeforme & Notothenia coriiceps & Isle Laurie & Szidat (1965) \\
\hline 834 & & & Lepocreadium trullaeforme & Notothenia coriiceps & Orcadas Del Sur & Szidat (1965) \\
\hline 835 & & & Lepocreadium trullaeforme & Notothenia coriiceps & Archipelago Melcihor & Szidat (1965) \\
\hline 836 & & & Lepocreadium trullaeforme & Notothenia coriiceps & South Shetland Islands & Szidat \& Graefe (1967) \\
\hline 837 & DIGENEA & & Lepocreadium trullaeforme & Notothenia rossii & Kerguelen Subregion & Parukhin \& Lyadov (1981) \\
\hline 838 & & & Lepocreadium trullaeforme & Notothenia rossii & Kerguelen Subregion & Parukhin \& Lyadov (1982) \\
\hline 839 & & & Lepocreadium trullaeforme & Notothenia rossii & Ob Bank & Parukhin (1986) \\
\hline 840 & & & Lepocreadium trullaeforme & Parachaenichthys charcoti & South Shetland Islands & Szidat \& Graefe (1967) \\
\hline 841 & & & Lepocreadium trullaeforme & HOST NAME NOT MENTIONED & Patagonian Shelf & Lyadov (1985) \\
\hline 842 & & & Lepocreadium trullaeforme & HOST NAME NOT MENTIONED & Glacial Subregion & Lyadov (1985) \\
\hline 843 & & & Lepocreadium trullaeforme & HOST NAME NOT MENTIONED & Kerguelen Subregion & Lyadov (1985) \\
\hline
\end{tabular}




\begin{tabular}{|c|c|c|c|c|c|c|}
\hline Index & Phylum & Family & Species & Host & Location & References \\
\hline 844 & & & Lepocreadium trullaeforme & HOST NAME NOT MENTIONED & Subantarctic Region & Zdzitowiecki (1990b) \\
\hline 845 & & & Muraenolepitrema magnatestis & blank & blank & Gaevskaya \& Rodjuk (1988) \\
\hline 846 & & & Muraenolepitrema magnatestis & Muraenolepis microps & South Georgia Island & Zdzitowiecki (1991) \\
\hline 847 & & & Muraenolepitrema magnatestis & Muraenolepis microps & South Georgia Island & Zdzitowiecki (1993) \\
\hline 848 & & & Neolepidapedon sp.indet. & blank & blank & Manter (1954) \\
\hline 849 & & & Neolepidapedon sp.indet. & Lepidonotothen mizops & $49^{\circ} 28^{\prime} \mathrm{S} 70^{\circ} 33^{\prime} \mathrm{E}$ & Prudhoe \& Bray (1973) \\
\hline 850 & & & Neolepidapedon sp.indet. & Lepidonotothen mizops & $\begin{array}{l}\text { Off Entrance To Royal } \\
\text { Sound (Kerguelen } \\
\text { Subregion) }\end{array}$ & Prudhoe \& Bray (1973) \\
\hline 851 & & & Neolepidapedon sp.indet. & Notothenia rossii & A.A.E. & Prudhoe \& Bray (1973) \\
\hline 852 & & & Neolepidapedon sp.indet. & Notothenia rossii & Macquarie Island & Prudhoe \& Bray (1973) \\
\hline 853 & & & Neolepidapedon sp.indet. & Notothenia macrocephala & A.A.E. & Prudhoe \& Bray (1973) \\
\hline 854 & & & Neolepidapedon sp.indet. & Notothenia macrocephala & Macquarie Island & Prudhoe \& Bray (1973) \\
\hline 855 & & & Neolepidapedon sp.indet. & Notothenia colbecki & A.A.E. & Prudhoe \& Bray (1973) \\
\hline 856 & & & Neolepidapedon sp.indet. & Notothenia colbecki & Macquarie Island & Prudhoe \& Bray (1973) \\
\hline 857 & & & Neolepidapedon sp.indet. & Trematomus newnesi & $66^{\circ} 45^{\prime} \mathrm{S} 64^{\circ} 52^{\prime} \mathrm{E}$ & Prudhoe \& Bray (1973) \\
\hline 858 & & & Neolepidapedon antarcticum & blank & blank & Prudhoe \& Bray (1973) \\
\hline 859 & \multirow{11}{*}{ DIGENEA } & & Neolepidapedon antarcticum & Macrourus whitsoni & $66^{\circ} 21^{\prime} \mathrm{S} 58^{\circ} 50^{\prime} \mathrm{E}$ & Prudhoe \& Bray (1973) \\
\hline 860 & & Lepocreadiidae & Neolepidapedon antarcticum & HOST NAME NOT MENTIONED & Glacial Subregion & Lyadov (1985) \\
\hline 861 & & & Neolepidapedon dubium & blank & blank & Prudhoe \& Bray (1973) \\
\hline 862 & & & Neolepidapedon dubium & Macrourus whitsoni & $66^{\circ} 21^{\prime} \mathrm{S} 58^{\circ} 50^{\prime} \mathrm{E}$ & Prudhoe \& Bray (1973) \\
\hline 863 & & & Neolepidapedon dubium & HOST NAME NOT MENTIONED & Glacial Subregion & Lyadov (1985) \\
\hline 864 & & & Neolepidapedon helicoleni & blank & blank & Prudhoe \& Bray (1973) \\
\hline 865 & & & Neolepidapedon helicoleni & Helicolenus percoides & $42^{\circ} 40^{\prime} \mathrm{S} 148^{\circ} 27^{\prime} 30^{\prime \prime} \mathrm{E}$ & Prudhoe \& Bray (1973) \\
\hline 866 & & & Neolepidapedon helicoleni & Helicolenus percoides & Off Maria, Tasmania & Prudhoe \& Bray (1973) \\
\hline 867 & & & Neolepidapedon helicoleni & Helicolenus percoides & $42^{\circ} 40^{\prime} \mathrm{S} 148^{\circ} 27^{\prime} 30^{\prime \prime} \mathrm{E}$ & Prudhoe \& Bray (1973) \\
\hline 868 & & & Neolepidapedon helicoleni & Scorpaena cruenta & Off Maria, Tasmania & Prudhoe \& Bray (1973) \\
\hline 869 & & & Neolepidapedon helicoleni & HOST NAME NOT MENTIONED & South Australia & Lyadov (1985) \\
\hline
\end{tabular}




\begin{tabular}{|c|c|c|c|c|c|c|}
\hline Index & Phylum & Family & Species & Host & Location & References \\
\hline 870 & & & Neolepidapedon magnatestis & blank & blank & Gaevskaya \& Kovaljova (1986) \\
\hline 871 & & & Neolepidapedon magnatestis & Dissostichus eleginoides & South Georgia Island & Zdzitowiecki (1990a) \\
\hline 872 & & & Neolepidapedon magnatestis & Dissostichus eleginoides & Shag Rocks & Zdzitowiecki (1990a) \\
\hline 873 & & & Neolepidapedon magnatestis & Dissostichus eleginoides & Shag Rocks & Brickle et al. (2005) \\
\hline 874 & & & Neolepidapedon magnatestis & Dissostichus eleginoides & South Georgia Island & Brickle et al. (2005) \\
\hline 875 & & & Neolepidapedon magnatestis & Dissostichus eleginoides & Prince Edward Island & Brickle et al. (2005) \\
\hline 876 & & & Neolepidapedon magnatestis & Dissostichus eleginoides & Ross Sea & Brickle et al. (2005) \\
\hline 877 & & & Neolepidapedon magnatestis & Dissostichus eleginoides & Falkland Islands & Brickle et al. (2006) \\
\hline 878 & & & Neolepidapedon magnatestis & Lepidonotothen squamifrons & South Georgia Island & Zdzitowiecki (1991) \\
\hline 879 & & & Neolepidapedon magnatestis & Lepidonotothen macrophthalma & North Scotia Ridge & Zdzitowiecki (1999) \\
\hline 880 & & & Neolepidapedon magnatestis & Lepidonotothen squamifrons & South Georgia Island & Zdzitowiecki (1999) \\
\hline 881 & & & Neolepidapedon magnatestis & Notothenia squamifrons & South Georgia Island & Zdzitowiecki (1990a) \\
\hline 882 & & & Neolepidapedon magnatestis & Notothenia squamifrons & Shag Rocks & Zdzitowiecki (1990a) \\
\hline 883 & & & Neolepidapedon opisthobifurcatus & blank & blank & Zdzitowiecki (1990) \\
\hline 884 & & & Neolepidapedon opisthobifurcatus & Muraenolepis microps & South Georgia Island & Zdzitowiecki (1990a) \\
\hline 885 & & & Neolepidapedon opisthobifurcatus & Muraenolepis microps & South Georgia Island & Zdzitowiecki (1991) \\
\hline 886 & & & Neolepidapedon trematomi & blank & blank & Prudhoe \& Bray (1973) \\
\hline 887 & & & Neolepidapedon trematomi & Austrolycichthys brachycephalus & A.A.E. & Prudhoe \& Bray (1973) \\
\hline 888 & & & Neolepidapedon trematomi & Austrolycichthys brachycephalus & $65^{\circ} 6^{\prime} \mathrm{S} 95^{\circ} 27^{\prime} \mathrm{E}$ & Prudhoe \& Bray (1973) \\
\hline 889 & \multirow{8}{*}{ DIGENEA } & Lepocreadiidae & Neolepidapedon trematomi & Dissostichus eleginoides & Shag Rocks & Zdzitowiecki (1999) \\
\hline 890 & & & Neolepidapedon trematomi & Dissostichus mawsoni & South Shetland Islands & Zdzitowiecki (1999) \\
\hline 891 & & & Neolepidapedon trematomi & Dissostichus mawsoni & South Shetland Islands & Zdzitowiecki (2002b) \\
\hline 892 & & & Neolepidapedon trematomi & Notothenia coriiceps & Admiralty Bay & $\begin{array}{c}\text { Zdzitowiecki \& Laskowski } \\
\text { (2004) }\end{array}$ \\
\hline 893 & & & Neolepidapedon trematomi & Notothenia coriiceps & Vernadsky Station & $\begin{array}{l}\text { Zdzitowiecki \& Laskowski } \\
\text { (2004) }\end{array}$ \\
\hline 894 & & & Neolepidapedon trematomi & Notothenia rossii & South Georgia Island & Zdzitowiecki (1990a) \\
\hline 895 & & & Neolepidapedon trematomi & Notothenia rossii & South Georgia Island & Zdzitowiecki (1991) \\
\hline 896 & & & Neolepidapedon trematomi & Pagothenia hansoni & South Georgia Island & Zdzitowiecki (1991) \\
\hline
\end{tabular}




\begin{tabular}{|c|c|c|c|c|c|c|}
\hline Index & Phylum & Family & Species & Host & Location & References \\
\hline 897 & & & Neolepidapedon trematomi & Pogonophryne permitini & Weddell Sea & Zdzitowiecki \& Cielecka (1997c) \\
\hline 898 & & & Neolepidapedon trematomi & Pogonophryne permitini & Weddell Sea & Zdzitowiecki (2002c) \\
\hline 899 & & & Neolepidapedon trematomi & Trematomus hansoni & A.A.E. & Prudhoe \& Bray (1973) \\
\hline 900 & & & Neolepidapedon trematomi & Trematomus hansoni & $65^{\circ} 42^{\prime} \mathrm{S} 92^{\circ} 10^{\prime} \mathrm{E}$ & Prudhoe \& Bray (1973) \\
\hline 901 & & & Neolepidapedon trematomi & Trematomus hansoni & South Georgia Island & Zdzitowiecki (1990a) \\
\hline 902 & & & Neolepidapedon trematomi & Trematomus hansoni & Weddell Sea & Zdzitowiecki \& Cielecka (1997c) \\
\hline 903 & & & Neolepidapedon trematomi & Trematomus hansoni & Weddell Sea & Zdzitowiecki (2002b) \\
\hline 904 & & & Neolepidapedon trematomi & Trematomus hansoni & $\begin{array}{l}\text { South Shetland Islands } \\
\text { (Admiralty Bay) }\end{array}$ & Zdzitowiecki (1999) \\
\hline 905 & & & Neolepidapedon trematomi & Trematomus hansoni & Adelie Land & Zdzitowiecki (2001b) \\
\hline 906 & & & Neolepidapedon trematomi & Trematomus hansoni & South Shetland Islands & Zdzitowiecki (2002b) \\
\hline 907 & & & Neolepidapedon trematomi & Trematomus loennbergi & Weddell Sea & Zdzitowiecki \& Cielecka (1997c) \\
\hline 908 & & & Neolepidapedon trematomi & Trematomus loennbergi & Weddell Sea & Zdzitowiecki (2002b) \\
\hline 909 & & & Neolepidapedon trematomi & Trematomus pennellii & Weddell Sea & Zdzitowiecki \& Cielecka (1997c) \\
\hline 910 & & & Neolepidapedon trematomi & Trematomus pennellii & Weddell Sea & Zdzitowiecki (2002b) \\
\hline 911 & & & Neolepidapedon trematomi & Trematomus scotti & A.A.E. & Prudhoe \& Bray (1973) \\
\hline 912 & & & Neolepidapedon trematomi & Trematomus scotti & $65^{\circ} 20^{\prime} \mathrm{S} 95^{\circ} 27^{\prime} \mathrm{E}$ & Prudhoe \& Bray (1973) \\
\hline 913 & & & Neolepidapedon trematomi & HOST NAME NOT MENTIONED & Glacial Subregion & Lyadov (1985) \\
\hline 914 & & & Opechona magnatestis & blank & blank & $\begin{array}{c}\text { Gaevskaya \& Kovaleva }(1976)= \\
\text { Neolepidapedon magnatestis }\end{array}$ \\
\hline 915 & & & Opechona magnatestis & Dissostichus eleginoides & Ob Bank & Parukhin (1986) \\
\hline 916 & & & Opechona magnatestis & Dissostichus eleginoides & Falkland-Patagonian Shelf & Gaevskaya et al. (1990) \\
\hline 917 & & & Opechona magnatestis & Dissostichus eleginoides & South Georgia Island & Gaevskaya et al. (1990) \\
\hline 918 & \multirow{6}{*}{ DIGENEA } & Lepocreadiidae & Opechona magnatestis & Gobionotothen acuta & Kerguelen Subregion & Parukhin \& Lyadov (1981) \\
\hline 919 & & & Opechona magnatestis & Gobionotothen acuta & Kerguelen Subregion & Parukhin \& Lyadov (1982) \\
\hline 920 & & & Opechona magnatestis & Lepidonotothen mizops & Kerguelen Subregion & Parukhin \& Lyadov (1981) \\
\hline 921 & & & Opechona magnatestis & Lepidonotothen mizops & Kerguelen Subregion & Parukhin \& Lyadov (1982) \\
\hline 922 & & & Opechona magnatestis & Lepidonotothen squamifrons & Kerguelen Subregion & Parukhin \& Lyadov (1981) \\
\hline 923 & & & Opechona magnatestis & Lepidonotothen squamifrons & Crozet Islands & Parukhin \& Lyadov (1981) \\
\hline
\end{tabular}




\begin{tabular}{|c|c|c|c|c|c|c|}
\hline Index & Phylum & Family & Species & Host & Location & References \\
\hline 924 & & & Opechona magnatestis & Lepidonotothen squamifrons & Lena Bank & Parukhin \& Lyadov (1981) \\
\hline 925 & & & Opechona magnatestis & Lepidonotothen squamifrons & Kerguelen Subregion & Parukhin \& Lyadov (1982) \\
\hline 926 & & & Opechona magnatestis & Lepidonotothen squamifrons & Crozet Islands & Parukhin \& Lyadov (1982) \\
\hline 927 & & & Opechona magnatestis & Lepidonotothen squamifrons & Ob Bank & Parukhin (1986) \\
\hline 928 & & & Opechona magnatestis & Nototheniidae & Kerguelen Subregion & Parukhin \& Lyadov (1982) \\
\hline 929 & & & Opechona magnatestis & Notothenia rossii & Lena Bank & Parukhin (1986) \\
\hline 930 & & & Opechona magnatestis & Notothenia rossii & Ob Bank & Parukhin (1986) \\
\hline 931 & & & Opechona magnatestis & Nototheniops larseni & Lena Bank & Parukhin (1986) \\
\hline 932 & & & Opechona magnatestis & Nototheniops larseni & Ob Bank & Parukhin (1986) \\
\hline 933 & & & Opechona magnatestis & Patagonotothen ramsayi & South Western Atlantic & Gaevskaya \& Kovaleva (1978) \\
\hline 934 & & & Opechona magnatestis & HOST NAME NOT MENTIONED & Glacial Subregion & Lyadov (1985) \\
\hline 935 & & & Opechona magnatestis & HOST NAME NOT MENTIONED & Kerguelen Subregion & Lyadov (1985) \\
\hline 936 & & & Paralepidapedon awii & blank & blank & Zdzitowiecki \& Cielecka (1997) \\
\hline 937 & & & Paralepidapedon awii & Macrourus whitsoni & Weddell Sea & Zdzitowiecki \& Cielecka (1997a) \\
\hline 938 & & & Paralepidapedon awii & Macrourus whitsoni & Weddell Sea & Zdzitowiecki (2003) \\
\hline 939 & & & Paralepidapedon lepidum & blank & blank & Gaevskaya \& Rodjuk (1988) \\
\hline 940 & & & Paralepidapedon lepidum & Macrourus holotrachys & North Scotia Ridge & Zdzitowiecki \& Cielecka (1998) \\
\hline 941 & & & Postlepidapedon opisthobifurcatus & blank & blank & Zdzitowiecki (1990) \\
\hline 942 & & & Postlepidapedon opisthobifurcatus & Macrourus whitsoni & Weddell Sea & Zdzitowiecki \& Cielecka (1997a) \\
\hline 943 & & & Postlepidapedon opisthobifurcatus & Macrourus whitsoni & Weddell Sea & Zdzitowiecki (2003) \\
\hline 944 & & & Postlepidapedon opisthobifurcatus & Muraenolepis microps & South Georgia Island & Zdzitowiecki (1993) \\
\hline 945 & & Monorchiidae & Postmonorchis variabilis & blank & blank & Prudhoe \& Bray (1973) \\
\hline 946 & & & Postmonorchis variabilis & Gobionotothen acuta & $\begin{array}{l}\text { Near Head of Bras } \\
\text { Bossiere (Kerguelen } \\
\text { Subregion) }\end{array}$ & Prudhoe \& Bray (1973) \\
\hline 947 & & Monorchiidae & Postmonorchis variabilis & Harpagifer bispinis & Port Jeanne d'Arc & Prudhoe \& Bray (1973) \\
\hline 948 & DIGENEA & & Postmonorchis variabilis & Harpagifer bispinis & Kerguelen Subregion & Prudhoe \& Bray (1973) \\
\hline 949 & & & Postmonorchis variabilis & Lepidonototnen mizops & Kerguelen Subregion & Parukhin \& Lyadov (1981) \\
\hline
\end{tabular}




\begin{tabular}{|c|c|c|c|c|c|c|}
\hline Index & Phylum & Family & Species & Host & Location & References \\
\hline 950 & & & Postmonorchis variabilis & Lepidonototnen mizops & Kerguelen Subregion & Parukhin \& Lyadov (1982) \\
\hline 951 & & & Postmonorchis variabilis & Lepidonotothen squamifrons & Lena Bank & Parukhin (1986) \\
\hline 952 & & & Postmonorchis variabilis & Lepidonotothen squamifrons & Ob Bank & Parukhin (1986) \\
\hline 953 & & & Postmonorchis variabilis & Lindbergichthys nudifrons & South Georgia Island & Zdzitowiecki (1991) \\
\hline 954 & & & Postmonorchis variabilis & Nototheniidae & Kerguelen Subregion & Parukhin \& Lyadov (1982) \\
\hline 955 & & & Postmonorchis variabilis & Notothenia cyanobrancha & Port Jeanne d'Arc & Prudhoe \& Bray (1973) \\
\hline 956 & & & Postmonorchis variabilis & Notothenia cyanobrancha & Kerguelen Subregion & Prudhoe \& Bray (1973) \\
\hline 957 & & & Postmonorchis variabilis & Notothenia rossii & $49^{\circ} 28^{\prime} \mathrm{S} 70^{\circ} 33^{\prime} \mathrm{E}$ & Prudhoe \& Bray (1973) \\
\hline 958 & & & Postmonorchis variabilis & Notothenia rossii & $\begin{array}{l}\text { Off Entrance To Royal } \\
\text { Sound (Kerguelen } \\
\text { Subregion) }\end{array}$ & Prudhoe \& Bray (1973) \\
\hline 959 & & & Postmonorchis variabilis & HOST NAME NOT MENTIONED & Glacial Subregion & Lyadov (1985) \\
\hline 960 & & & Postmonorchis variabilis & HOST NAME NOT MENTIONED & Kerguelen Subregion & Lyadov (1985) \\
\hline 961 & & Opecoelidae & Opecolidae sp. indet. & Chionodraco kathleenae & A.A.E. & Prudhoe \& Bray (1973) \\
\hline 962 & & & Opecolidae sp. indet. & Chionodraco kathleenae & $65^{\circ} 6^{\prime} \mathrm{S} 96^{\circ} 14^{\prime} \mathrm{E}$ & Prudhoe \& Bray (1973) \\
\hline 963 & & & Allocreadium fowleri & blank & blank & $\begin{array}{c}\text { Leiper \& Atkinson }(1914)= \\
\quad \text { Macvicaria pennellii }\end{array}$ \\
\hline 964 & & & Allocreadium fowleri & Trematomus bernacchii & $\begin{array}{c}\text { Ross Sea (Terra Nova } \\
\text { Bay) }\end{array}$ & Zdzitowiecki et al. (1992) \\
\hline 965 & & & Allocreadium fowleri & Trematomus centronotus $=T$. pennellii & $\begin{array}{c}\text { Ross Sea (Terra Nova } \\
\text { Bay) }\end{array}$ & Zdzitowiecki et al. (1992) \\
\hline 966 & & & Discoverytrema gibsoni & blank & blank & Zdzitowiecki (1990) \\
\hline 967 & & & Discoverytrema gibsoni & Lindbergichthys nudifrons & South Georgia Island & Zdzitowiecki (1991) \\
\hline 968 & & & Discoverytrema gibsoni & Muraenolepis microps & South Georgia Island & Zdzitowiecki (1991) \\
\hline 969 & & & Discoverytrema gibsoni & Muraenolepis microps & South Shetland Islands & Palm et al. (2007) \\
\hline 970 & & & Discoverytrema markowskii & blank & blank & Gibson (1976) \\
\hline 971 & & & Discoverytrema markowskii & HOST NAME NOT MENTIONED & Glacial Subregion & Lyadov (1985) \\
\hline 972 & & & Discoverytrema markowskii & HOST NAME NOT MENTIONED & Kerguelen Subregion & Lyadov (1985) \\
\hline 973 & & & Discoverytrema markowskii & Muraenolepis microps & South Shetland Islands & Palm et al. (2007) \\
\hline 974 & & & Helicometra sp. indet. & blank & blank & Odhner (1902) \\
\hline 975 & DIGENEA & Opecoelidae & Helicometra sp. indet. & Trematomus hansoni & A.A.E. & Prudhoe \& Bray (1973) \\
\hline
\end{tabular}




\begin{tabular}{|c|c|c|c|c|c|c|}
\hline Index & Phylum & Family & Species & Host & Location & References \\
\hline 976 & & & Helicometra sp. indet. & Trematomus hansoni & $65^{\circ} 42^{\prime} \mathrm{S} 92^{\circ} 10^{\prime} \mathrm{E}$ & Prudhoe \& Bray (1973) \\
\hline 977 & & & Helicometra sp. indet. & Trematomus loennbergi & A.A.E. & Prudhoe \& Bray (1973) \\
\hline 978 & & & Helicometra sp. indet. & Trematomus loennbergi & $65^{\circ} 20^{\prime} \mathrm{S} 95^{\circ} 27^{\prime} \mathrm{E}$ & Prudhoe \& Bray (1973) \\
\hline 979 & & & Helicometra sp. indet. & Trematomus scotti & A.A.E. & Prudhoe \& Bray (1973) \\
\hline 980 & & & Helicometra sp. indet. & Trematomus scotti & $65^{\circ} 42^{\prime} \mathrm{S} 92^{\circ} 10^{\prime} \mathrm{E}$ & Prudhoe \& Bray (1973) \\
\hline 981 & & & Helicometra antarcticae & blank & blank & Holloway \& Bier (1968) \\
\hline 982 & & & Helicometra antarcticae & Dissostichus eleginoides & Ross Sea & Brickle et al. (2005) \\
\hline 983 & & & Helicometra pisanoae & blank & blank & Zdzitowiecki (1998) \\
\hline 984 & & & Helicometra pisanoae & Trematomus hansoni & Adelie Land & Zdzitowiecki et.al. (1998) \\
\hline 985 & & & Helicometra pisanoae & Trematomus hansoni & Adelie Land & Zdzitowiecki (1998) \\
\hline 986 & & & Helicometra pisanoae & Trematomus hansoni & Adelie Land & Zdzitowiecki (2001b) \\
\hline 987 & & & Helicometra rakusai & blank & blank & Zdzitowiecki (1997) \\
\hline 988 & & & Helicometra rakusai & Trematomus loennbergi & Weddell Sea & Zdzitowiecki (1997a) \\
\hline 989 & & & Helicometra rakusai & Trematomus loennbergi & Weddell Sea & Zdzitowiecki (2002b) \\
\hline 990 & & & Helicometra scorpaenae & blank & blank & Prudhoe \& Bray (1973) \\
\hline 991 & & & Helicometra scorpaenae & Scorpaena cruenta & $42^{\circ} 40^{\prime} \mathrm{S} 148^{\circ} 27^{\prime} 30^{\prime \prime} \mathrm{E}$ & Prudhoe \& Bray (1973) \\
\hline 992 & & & Helicometra scorpaenae & Scorpaena cruenta & Off Maria, Tasmania & Prudhoe \& Bray (1973) \\
\hline 993 & & & Helicometra scorpaenae & HOST NAME NOT MENTIONED & South Australia & Lyadov (1985) \\
\hline 994 & & & Macvicaria sp. $=$ Plagioporus $s p$ & blank & blank & Gibson \& Bray (1982) \\
\hline 995 & & & Macvicaria sp. =Plagioporus sp. & HOST NAME NOT MENTIONED & Kerguelen Subregion & Lyadov (1985) \\
\hline 996 & & & Macvicaria antarctica (also Plagioporus) & blank & blank & Kovaljova \& Gaevskaya (1974) \\
\hline 997 & & & Macvicaria antarctica (also Plagioporus) & Gobionotothen acuta & Kerguelen Subregion & Parukhin \& Lyadov (1981) \\
\hline 998 & & & Macvicaria antarctica (also Plagioporus) & Gobionotothen acuta & Kerguelen Subregion & Parukhin \& Lyadov (1982) \\
\hline 999 & & & Macvicaria antarctica (also Plagioporus) & Lepidonotothen macrophthalma & North Scotia Ridge & Zdzitowiecki (1999) \\
\hline 1000 & & & Macvicaria antarctica (also Plagioporus) & Lepidonotothen mizops & Kerguelen Subregion & Parukhin \& Lyadov (1981) \\
\hline 1001 & & & Macvicaria antarctica (also Plagioporus) & Lepidonotothen mizops & Kerguelen Subregion & Parukhin \& Lyadov (1982) \\
\hline 1002 & & & Macvicaria antarctica (also Plagioporus) & Lindbergichthys nudifrons & South Georgia Island & Zdzitowiecki (1991) \\
\hline
\end{tabular}




\begin{tabular}{|c|c|c|c|c|c|c|}
\hline Index & Phylum & Family & Species & Host & Location & References \\
\hline 1003 & & & Macvicaria antarctica (also Plagioporus) & Lepidonotothen squamifrons & Kerguelen Subregion & Parukhin \& Lyadov (1981) \\
\hline 1004 & \multirow{25}{*}{ DIGENEA } & Opecoelidae & Macvicaria antarctica (also Plagioporus) & Lepidonotothen squamifrons & Crozet Islands & Parukhin \& Lyadov (1982) \\
\hline 1005 & & & Macvicaria antarctica (also Plagioporus) & Lepidonotothen squamifrons & Ob Bank & Parukhin \& Lyadov (1982) \\
\hline 1006 & & & Macvicaria antarctica (also Plagioporus) & Lepidonotothen squamifrons & Lena Bank & Parukhin \& Lyadov (1982) \\
\hline 1007 & & & Macvicaria antarctica (also Plagioporus) & Lepidonotothen squamifrons & Lena Bank & Parukhin (1986) \\
\hline 1008 & & & Macvicaria antarctica (also Plagioporus) & Nototheniidae & Kerguelen Subregion & Parukhin \& Lyadov (1982) \\
\hline 1009 & & & Macvicaria antarctica (also Plagioporus) & HOST NAME NOT MENTIONED & Glacial Subregion & Lyadov (1985) \\
\hline 1010 & & & Macvicaria antarctica (also Plagioporus) & HOST NAME NOT MENTIONED & Kerguelen Subregion & Lyadov (1985) \\
\hline 1011 & & & Macvicaria antarctica (also Plagioporus) & HOST NAME NOT MENTIONED & Patagonian Shelf & Lyadov (1985) \\
\hline 1012 & & & Macvicaria georgiana & blank & blank & Kovaljova \& Gaevskaya (1974) \\
\hline 1013 & & & Macvicaria georgiana & Cryodraco antarcticus & Weddell Sea & Zdzitowiecki \& Cielecka (1997b) \\
\hline 1014 & & & Macvicaria georgiana & Cryodraco antarcticus & Weddell Sea & Zdzitowiecki (2002a) \\
\hline 1015 & & & Macvicaria georgiana & Gobionotothen gibberifrons & Signy Island & Zdzitowiecki et al. (1997) \\
\hline 1016 & & & Macvicaria georgiana & Gobionotothen gibberifrons & South Orkney Islands & Zdzitowiecki et al. (1997) \\
\hline 1017 & & & Macvicaria georgiana & Harpagifer antarcticus & Vernadsky Station & $\begin{array}{l}\text { Laskowski \& Zdzitowiecki } \\
\text { (2005) }\end{array}$ \\
\hline 1018 & & & Macvicaria georgiana & Harpagifer georgianus & Off Elephant Island & Zdzitowiecki \& Zadrozny (1999) \\
\hline 1019 & & & Macvicaria georgiana & Lepidonotothen larseni & $\begin{array}{l}\text { Port Foster (Deception } \\
\text { Island) }\end{array}$ & Ruhl et al. (2003) \\
\hline 1020 & & & Macvicaria georgiana & Lindbergichthys nudifrons & Vernadsky Station & $\begin{array}{c}\text { Laskowski \& Zdzitowiecki } \\
\text { (2005) }\end{array}$ \\
\hline 1021 & & & Macvicaria georgiana & Lindbergichthys nudifrons & Admiralty Bay & $\begin{array}{l}\text { Laskowski \& Zdzitowiecki } \\
\text { (2005) }\end{array}$ \\
\hline 1022 & & & Macvicaria georgiana & Notothenia coriiceps & Signy Island & Zdzitowiecki et al. (1997) \\
\hline 1023 & & & Macvicaria georgiana & Notothenia coriiceps & South Orkney Islands & Zdzitowiecki et al. (1997) \\
\hline 1024 & & & Macvicaria georgiana & Notothenia coriiceps & South Shetland Islands & Zdzitowiecki (2002b) \\
\hline 1025 & & & Macvicaria georgiana & Notothenia coriiceps & Admiralty Bay & $\begin{array}{l}\text { Zdzitowiecki \& Laskowski } \\
\text { (2004) }\end{array}$ \\
\hline 1026 & & & Macvicaria georgiana & Notothenia coriiceps & Vernadsky Station & $\begin{array}{l}\text { Zdzitowiecki \& Laskowski } \\
\text { (2004) }\end{array}$ \\
\hline 1027 & & & Macvicaria georgiana & Parachaenichthys charcoti & Admiralty Bay & Zdzitowiecki (2001a) \\
\hline 1028 & & & Macvicaria georgiana & Trematomus bernacchii & Weddell Sea & Zdzitowiecki \& Cielecka (1997b) \\
\hline
\end{tabular}




\begin{tabular}{|c|c|c|c|c|c|c|}
\hline Index & Phylum & Family & Species & Host & Location & References \\
\hline 1029 & \multirow{26}{*}{ DIGENEA } & & Macvicaria georgiana & Trematomus bernacchii & Vernadsky Station & $\begin{array}{l}\text { Laskowski \& Zdzitowiecki } \\
\qquad(2005)\end{array}$ \\
\hline 1030 & & & Macvicaria georgiana & Trematomus bernacchii & Admiralty Bay & $\begin{array}{c}\text { Laskowski \& Zdzitowiecki } \\
\text { (2005) }\end{array}$ \\
\hline 1031 & & & Macvicaria georgiana & Trematomus eulepidotus & Weddell Sea & Zdzitowiecki \& Cielecka (1997b) \\
\hline 1032 & & Opecoelidae & Macvicaria georgiana & Trematomus eulepidotus & Weddell Sea & Zdzitowiecki (2002b) \\
\hline 1033 & & & Macvicaria georgiana & Trematomus hansoni & Weddell Sea & Zdzitowiecki \& Cielecka (1997b) \\
\hline 1034 & & & Macvicaria georgiana & Trematomus hansoni & Weddell Sea & Zdzitowiecki (2002b) \\
\hline 1035 & & & Macvicaria georgiana & Trematomus lepidorhinus & Weddell Sea & Zdzitowiecki \& Cielecka (1997b) \\
\hline 1036 & & & Macvicaria georgiana & Trematomus lepidorhinus & Weddell Sea & Zdzitowiecki (2002b) \\
\hline 1037 & & & Macvicaria georgiana & Trematomus loennbergi & Weddell Sea & Zdzitowiecki \& Cielecka (1997b) \\
\hline 1038 & & & Macvicaria georgiana & Trematomus loennbergi & Weddell Sea & Zdzitowiecki (2002b) \\
\hline 1039 & & & Macvicaria georgiana & Trematomus newnesi & Vernadsky Station & $\begin{array}{l}\text { Laskowski \& Zdzitowiecki } \\
\qquad(2005)\end{array}$ \\
\hline 1040 & & & Macvicaria georgiana & Trematomus newnesi & Admiralty Bay & $\begin{array}{c}\text { Laskowski \& Zdzitowiecki } \\
\text { (2005) }\end{array}$ \\
\hline 1041 & & & Macvicaria georgiana & Trematomus nicolai & Weddell Sea & Zdzitowiecki \& Cielecka (1997b) \\
\hline 1042 & & & Macvicaria georgiana & Trematomus nicolai & Weddell Sea & Zdzitowiecki (2002b) \\
\hline 1043 & & & Macvicaria georgiana & Trematomus pennellii & Weddell Sea & Zdzitowiecki \& Cielecka (1997b) \\
\hline 1044 & & & Macvicaria georgiana & Trematomus pennellii & Weddell Sea & Zdzitowiecki (2002b) \\
\hline 1045 & & & Macvicaria georgiana & Trematomus scotti & Weddell Sea & Zdzitowiecki \& Cielecka (1997b) \\
\hline 1046 & & & Macvicaria georgiana & Trematomus scotti & Weddell Sea & Zdzitowiecki (2002b) \\
\hline 1047 & & & Macvicaria lobata georgiana & blank & blank & Gaevskaya \& Kovaljova (1976) \\
\hline 1048 & & & Macvicaria lobata georgiana & Cheanocephalus aceratus & Weddell Sea & Parukhin \& Lyadov (1981) \\
\hline 1049 & & & Macvicaria lobata georgiana & Champsocephalus gunnari & South Georgia Island & Parukhin \& Lyadov (1981) \\
\hline 1050 & & & Macvicaria lobata georgiana & Dissostichus eleginoides & Shag Rocks & Parukhin \& Lyadov (1981) \\
\hline 1051 & & & Macvicaria lobata georgiana & Gobionotothen gibberifrons & Crozet Islands & Parukhin \& Lyadov (1981) \\
\hline 1052 & & & Macvicaria lobata georgiana & Lepidonotothen kempi & Shag Rocks & Parukhin \& Lyadov (1981) \\
\hline 1053 & & & Macvicaria lobata georgiana & Notothenia rossii & South Georgia Island & Parukhin \& Lyadov (1981) \\
\hline 1054 & & & Macvicaria lobata georgiana & Pseudochaenichthys georgianus & South Georgia Island & Parukhin \& Lyadov (1981) \\
\hline
\end{tabular}




\begin{tabular}{|c|c|c|c|c|c|c|}
\hline Index & Phylum & Family & Species & Host & Location & References \\
\hline 1055 & & & Macvicaria longibursata & blank & blank & Zdzitowiecki \& Cielecka (1997) \\
\hline 1056 & & & Macvicaria longibursata & Ophthalmolycus amberensis & Weddell Sea & Zdzitowiecki \& Cielecka (1997b) \\
\hline 1057 & & & Macvicaria longibursata & Ophthalmolycus amberensis & Weddell Sea & Zdzitowiecki (2003) \\
\hline 1058 & & & Macvicaria longibursata & Ophthalmolycus bothriocephalus & Weddell Sea & Zdzitowiecki \& Cielecka (1997b) \\
\hline 1059 & & & Macvicaria longibursata & Ophthalmolycus bothriocephalus & Weddell Sea & Zdzitowiecki (2003) \\
\hline 1060 & & & Macvicaria microtestis & Ophthalmolycus bothriocephalus & Weddell Sea & Zdzitowiecki \& Cielecka (1997) \\
\hline 1061 & \multirow{21}{*}{ DIGENEA } & Opecoelidae & Macvicaria microtestis & blank & blank & Zdzitowiecki \& Cielecka (1997b) \\
\hline 1062 & & & Macvicaria microtestis & Artedidraco loennbergi & Weddell Sea & Zdzitowiecki (2002c) \\
\hline 1063 & & & Macvicaria microtestis & Artedidraco orianae & Weddell Sea & Zdzitowiecki \& Cielecka (1997b) \\
\hline 1064 & & & Macvicaria microtestis & Artedidraco orianae & Weddell Sea & Zdzitowiecki (2002c) \\
\hline 1065 & & & Macvicaria microtestis & Artedidraco skottsbergi & Weddell Sea & Zdzitowiecki \& Cielecka (1997b) \\
\hline 1066 & & & Macvicaria microtestis & Artedidraco skottsbergi & Weddell Sea & Zdzitowiecki (2002c) \\
\hline 1067 & & & Macvicaria microtestis & Dolloidraco longedorsalis & Weddell Sea & Zdzitowiecki \& Cielecka (1997b) \\
\hline 1068 & & & Macvicaria microtestis & Dolloidraco longedorsalis & Weddell Sea & Zdzitowiecki (2002c) \\
\hline 1069 & & & Macvicaria microtestis & Pogonophryne marmorata & Weddell Sea & Zdzitowiecki \& Cielecka (1997b) \\
\hline 1070 & & & Macvicaria microtestis & Pogonophryne marmorata & Weddell Sea & Zdzitowiecki (2002c) \\
\hline 1071 & & & Macvicaria microtestis & Notothenia coriiceps & Adelie Land & Zdzitowiecki et.al. (1998) \\
\hline 1072 & & & Macvicaria microtestis & Notothenia coriiceps & Adelie Land & Zdzitowiecki (2001b) \\
\hline 1073 & & & Macvicaria microtestis & Trematomus hansoni & Adelie Land & Zdzitowiecki et.al. (1998) \\
\hline 1074 & & & Macvicaria microtestis & Trematomus lepidorhinus & Weddell Sea & Zdzitowiecki \& Cielecka (1997b) \\
\hline 1075 & & & Macvicaria microtestis & Trematomus lepidorhinus & Weddell Sea & Zdzitowiecki (2002b) \\
\hline 1076 & & & Macvicaria microtestis & Trematomus loennbergi & Weddell Sea & Zdzitowiecki \& Cielecka (1997b) \\
\hline 1077 & & & Macvicaria microtestis & Trematomus loennbergi & Weddell Sea & Zdzitowiecki (2002b) \\
\hline 1078 & & & Macvicaria muraenolepidis & blank & blank & Zdzitowiecki (1990) \\
\hline 1079 & & & Macvicaria muraenolepidis & Muraenolepis microps & South Georgia Island & Zdzitowiecki (1991) \\
\hline 1080 & & & Macvicaria ophthalmolyci & blank & blank & Zdzitowiecki (1990) \\
\hline 1081 & & & Macvicaria ophthalmolyci & Ophthalmolycus concolor & South Shetland Islands & Zdzitowiecki (1991) \\
\hline
\end{tabular}




\begin{tabular}{|c|c|c|c|c|c|c|}
\hline Index & Phylum & Family & Species & Host & Location & References \\
\hline 1082 & & & Macvicaria pennelli & blank & blank & Leiper \& Atkinson (1914) \\
\hline 1083 & & & Macvicaria pennelli & Ophthalmolycus concolor & South Shetland Islands & Zdzitowiecki (1990) \\
\hline 1084 & & & Macvicaria pennelli & Chaenocephalus aceratus & South Shetland Islands & Zdzitowiecki (1979) \\
\hline 1085 & & & Macvicaria pennelli & Champsocephalus gunnari & South Shetland Islands & Zdzitowiecki (1991) \\
\hline 1086 & & & Macvicaria pennelli & Cryodraco antarcticus & $65^{\circ} 42^{\prime} \mathrm{S} 92^{\circ} 10^{\prime} \mathrm{E}$ & Prudhoe \& Bray (1973) \\
\hline 1087 & & & Macvicaria pennelli & Dissostichus mawsoni & South Shetland Islands & Zdzitowiecki (1991) \\
\hline 1088 & & & Macvicaria pennelli & Gobionotothen gibberifrons & South Shetland Islands & Zdzitowiecki (1991) \\
\hline 1089 & & & Macvicaria pennelli & Harpagifer antarcticus & South Shetland Islands & Zdzitowiecki (1991) \\
\hline 1090 & \multirow{18}{*}{ DIGENEA } & Opecoelidae & Macvicaria pennelli & Nototheniidae & South Shetland Islands & Szidat \& Graefe (1967) \\
\hline 1091 & & & Macvicaria pennelli & Notothenia coriiceps & $\begin{array}{c}\text { Bhaia Scotia La Isla } \\
\text { Laurie (Orcadas del sur, } \\
\text { Argentina) }\end{array}$ & Szidat (1965) \\
\hline 1092 & & & Macvicaria pennelli & Notothenia coriiceps & Isle Laurie & Szidat (1965) \\
\hline 1093 & & & Macvicaria pennelli & Notothenia coriiceps & Orcadas Del Sur & Szidat (1965) \\
\hline 1094 & & & Macvicaria pennelli & Notothenia coriiceps & Archipelago Melcihor & Szidat (1965) \\
\hline 1095 & & & Macvicaria pennelli & Notothenia corriceps & South Shetland Islands & Zdzitowiecki (1979) \\
\hline 1096 & & & Macvicaria pennelli & Notothenia coriiceps & $\begin{array}{c}\text { Potter Cove (King George } \\
\text { Island) }\end{array}$ & Palm et.al. (1998) \\
\hline 1097 & & & Macvicaria pennelli & Notothenia coriiceps & King George Island & Palm et.al. (1998) \\
\hline 1098 & & & Macvicaria pennelli & Notothenia coriiceps & Adelie Land & Zdzitowiecki et.al. (1998) \\
\hline 1099 & & & Macvicaria pennelli & Notothenia coriiceps & Adelie Land & Zdzitowiecki (2001b) \\
\hline 1100 & & & Macvicaria pennelli & Notothenia rossii & South Shetland Islands & Zdzitowiecki (1979) \\
\hline 1101 & & & Macvicaria pennelli & Notothenia rossii & South Georgia Island & Zdzitowiecki (1979) \\
\hline 1102 & & & Macvicaria pennelli & Notothenia rossii & South Georgia Island & Zdzitowiecki (1991) \\
\hline 1103 & & & Macvicaria pennelli & Pleurogramma antarctica & South Shetland Islands & Zdzitowiecki (1979) \\
\hline 1104 & & & Macvicaria pennelli & Trematomus bernacchii & South Shetland Islands & Zdzitowiecki (1979) \\
\hline 1105 & & & Macvicaria pennelli & Trematomus bernacchii & $\begin{array}{c}\text { Ross Sea (Terra Nova } \\
\text { Bay) }\end{array}$ & Zdzitowiecki et al. (1992) \\
\hline 1106 & & & Macvicaria pennelli & Trematomus centronotus $=T$. pennellii & $\begin{array}{c}\text { Ross Sea (Terra Nova } \\
\text { Bay) }\end{array}$ & Zdzitowiecki et al. (1992) \\
\hline 1107 & & & Macvicaria pennelli & Trematomus eulepidotus & $66^{\circ} 45^{\prime} \mathrm{S} 62^{\circ} 03^{\prime} \mathrm{E}$ & Prudhoe \& Bray (1973) \\
\hline
\end{tabular}




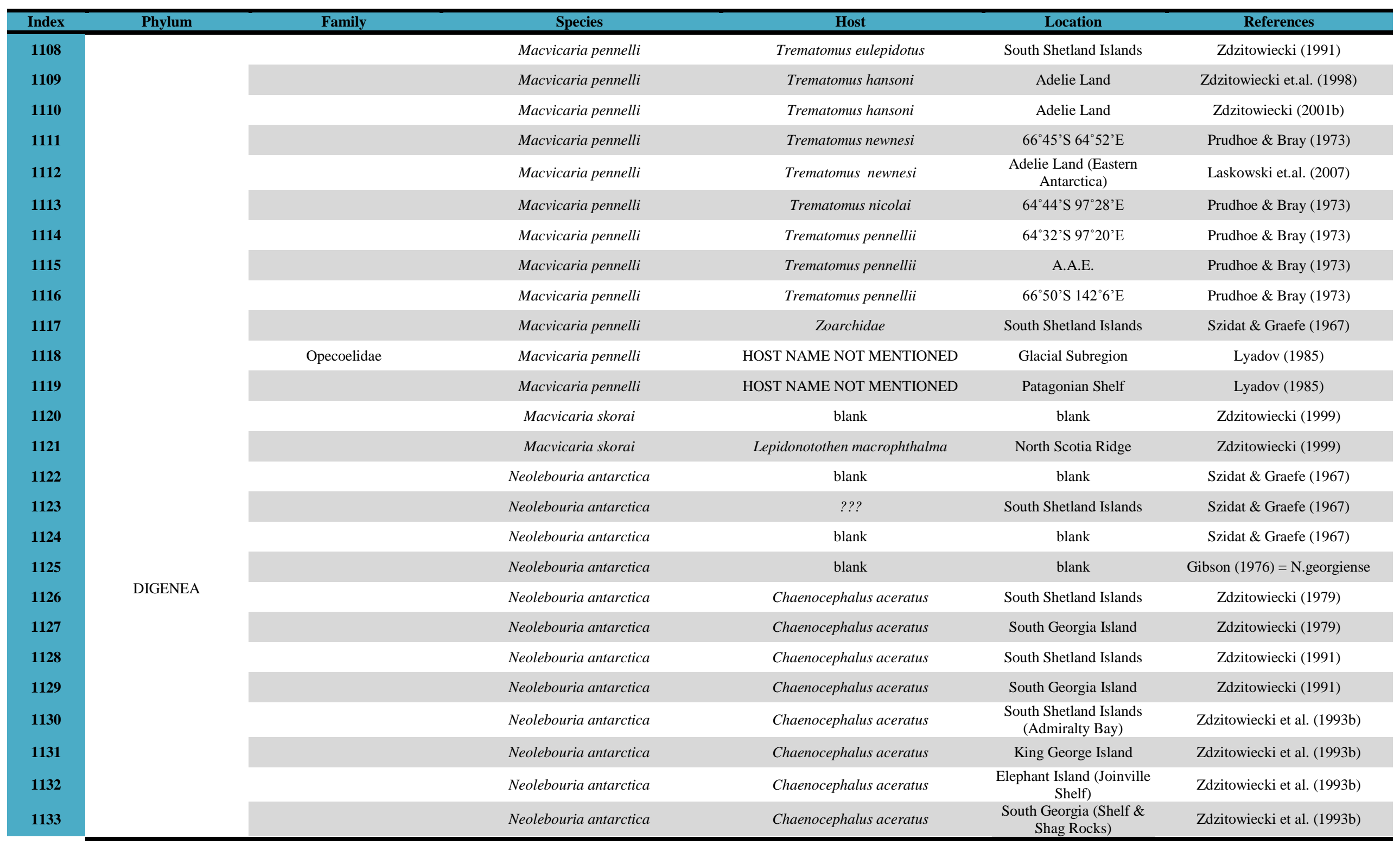




\begin{tabular}{|c|c|c|c|c|c|c|}
\hline Index & Phylum & Family & Species & Host & Location & References \\
\hline 1134 & & & Neolebouria antarctica & Chaenocephalus aceratus & Signy Island & Zdzitowiecki et al. (1997) \\
\hline 1135 & & & Neolebouria antarctica & Chaenocephalus aceratus & South Orkney Islands & Zdzitowiecki et al. (1997) \\
\hline 1136 & & & Neolebouria antarctica & Chaenocephalus aceratus & South Shetland Islands & Palm et al. (2007) \\
\hline 1137 & & & Neolebouria antarctica & Champsocephalus gunnari & South Shetland Islands & Zdzitowiecki (1991) \\
\hline 1138 & & & Neolebouria antarctica & Champsocephalus gunnari & South Georgia Island & Zdzitowiecki (1991) \\
\hline 1139 & & & Neolebouria antarctica & Champsocephalus gunnari & $\begin{array}{l}\text { South Shetland Islands } \\
\text { (Admiralty Bay) }\end{array}$ & Zdzitowiecki et al. (1993b) \\
\hline 1140 & & & Neolebouria antarctica & Champsocephalus gunnari & King George Island & Zdzitowiecki et al. (1993b) \\
\hline 1141 & & & Neolebouria antarctica & Champsocephalus gunnari & $\begin{array}{l}\text { Elephant Island (Joinville } \\
\text { Shelf) }\end{array}$ & Zdzitowiecki et al. (1993b) \\
\hline 1142 & & & Neolebouria antarctica & Champsocephalus gunnari & $\begin{array}{c}\text { South Georgia (Shelf \& } \\
\text { Shag Rocks) }\end{array}$ & Zdzitowiecki et al. (1993b) \\
\hline 1143 & & & Neolebouria antarctica & Champsocephalus gunnari & Admiralty Bay & Zdzitowiecki (2002a) \\
\hline 1144 & & & Neolebouria antarctica & Chionodraco rastrospinosus & South Shetland Islands & Zdzitowiecki (1991) \\
\hline 1145 & & & Neolebouria antarctica & Chiondraco rastrospinosus & $\begin{array}{l}\text { South Shetland Islands } \\
\text { (Admiralty Bay) }\end{array}$ & Zdzitowiecki et al. (1993b) \\
\hline 1146 & & Opecoelidae & Neolebouria antarctica & Chiondraco rastrospinosus & King George Island & Zdzitowiecki et al. (1993b) \\
\hline 1147 & & & Neolebouria antarctica & Chiondraco rastrospinosus & $\begin{array}{l}\text { Elephant Island (Joinville } \\
\text { Shelf) }\end{array}$ & Zdzitowiecki et al. (1993b) \\
\hline 1148 & & & Neolebouria antarctica & Chiondraco rastrospinosus & $\begin{array}{c}\text { South Georgia (Shelf \& } \\
\text { Shag Rocks) }\end{array}$ & Zdzitowiecki et al. (1993b) \\
\hline 1149 & & & Neolebouria antarctica & Cryodraco antarcticus & South Shetland Islands & Zdzitowiecki (1991) \\
\hline 1150 & & & Neolebouria antarctica & Cryodraco antarcticus & $\begin{array}{l}\text { South Shetland Islands } \\
\text { (Admiralty Bay) }\end{array}$ & Zdzitowiecki et al. (1993b) \\
\hline 1151 & & & Neolebouria antarctica & Cryodraco antarcticus & King George Island & Zdzitowiecki et al. (1993b) \\
\hline 1152 & DIGENEA & & Neolebouria antarctica & Cryodraco antarcticus & $\begin{array}{l}\text { Elephant Island (Joinville } \\
\text { Shelf) }\end{array}$ & Zdzitowiecki et al. (1993b) \\
\hline 1153 & & & Neolebouria antarctica & Cryodraco antarcticus & $\begin{array}{l}\text { South Georgia (Shelf \& } \\
\text { Shag Rocks) }\end{array}$ & Zdzitowiecki et al. (1993b) \\
\hline 1154 & & & Neolebouria antarctica & Cryodraco antarcticus & Admiralty Bay & Zdzitowiecki (2002a) \\
\hline 1155 & & & Neolebouria antarctica & Dissostichus eleginoides & South Georgia Island & Gaevskaya et al. (1990) \\
\hline 1156 & & & Neolebouria antarctica & Dissostichus eleginoides & $\begin{array}{l}\text { South Shetland Islands } \\
\text { (Admiralty Bay) }\end{array}$ & Zdzitowiecki et al. (1993b) \\
\hline 1157 & & & Neolebouria antarctica & Dissostichus eleginoides & King George Island & Zdzitowiecki et al. (1993b) \\
\hline 1158 & & & Neolebouria antarctica & Dissostichus eleginoides & $\begin{array}{c}\text { Elephant Island (Joinville } \\
\text { Shelf) }\end{array}$ & Zdzitowiecki et al. (1993b) \\
\hline
\end{tabular}




\begin{tabular}{|c|c|c|c|c|c|c|}
\hline Index & Phylum & Family & Species & Host & Location & References \\
\hline 1159 & & & Neolebouria antarctica & Dissostichus eleginoides & $\begin{array}{c}\text { South Georgia (Shelf \& } \\
\text { Shag Rocks) }\end{array}$ & Zdzitowiecki et al. (1993b) \\
\hline 1160 & & & Neolebouria antarctica & Dissostichus eleginoides & South Georgia Island & Brickle et al. (2005) \\
\hline 1161 & & & Neolebouria antarctica & Dissostichus eleginoides & Ross Sea & Brickle et al. (2005) \\
\hline 1162 & & & Neolebouria antarctica & Dissostichus mawsoni & $\begin{array}{l}\text { South Shetland Islands } \\
\text { (Admiralty Bay) }\end{array}$ & Zdzitowiecki et al. (1993b) \\
\hline 1163 & & & Neolebouria antarctica & Dissostichus mawsoni & King George Island & Zdzitowiecki et al. (1993b) \\
\hline 1164 & & & Neolebouria antarctica & Dissostichus mawsoni & $\begin{array}{c}\text { Elephant Island (Joinville } \\
\text { Shelf) }\end{array}$ & Zdzitowiecki et al. (1993b) \\
\hline 1165 & & & Neolebouria antarctica & Dissostichus mawsoni & $\begin{array}{c}\text { South Georgia (Shelf \& } \\
\text { Shag Rocks) }\end{array}$ & Zdzitowiecki et al. (1993b) \\
\hline 1166 & & & Neolebouria antarctica & Lepidonotothen kempi & $\begin{array}{l}\text { South Shetland Islands } \\
\text { (Admiralty Bay) }\end{array}$ & Zdzitowiecki et al. (1993b) \\
\hline 1167 & & & Neolebouria antarctica & Lepidonotothen kempi & King George Island & Zdzitowiecki et al. (1993b) \\
\hline 1168 & & & Neolebouria antarctica & Lepidonotothen kempi & $\begin{array}{l}\text { Elephant Island (Joinville } \\
\text { Shelf) }\end{array}$ & Zdzitowiecki et al. (1993b) \\
\hline 1169 & & & Neolebouria antarctica & Lepidonotothen kempi & $\begin{array}{l}\text { South Georgia (Shelf \& } \\
\text { Shag Rocks) }\end{array}$ & Zdzitowiecki et al. (1993b) \\
\hline 1170 & & & Neolebouria antarctica & Lepidonotothen larseni & $\begin{array}{c}\text { Port Foster (Deception } \\
\text { Island) }\end{array}$ & Ruhl et al. (2003) \\
\hline 1171 & & & Neolebouria antarctica & Lepidonotothen squamifrons & South Georgia Island & Zdzitowiecki (1991) \\
\hline 1172 & & & Neolebouria antarctica & Lepidonotothen squamifrons & $\begin{array}{l}\text { South Shetland Islands } \\
\text { (Admiralty Bay) }\end{array}$ & Zdzitowiecki et al. (1993b) \\
\hline 1173 & & Opecoelidae & Neolebouria antarctica & Lepidonotothen squamifrons & King George Island & Zdzitowiecki et al. (1993b) \\
\hline 1174 & & & Neolebouria antarctica & Lepidonotothen squamifrons & $\begin{array}{l}\text { Elephant Island (Joinville } \\
\text { Shelf) }\end{array}$ & Zdzitowiecki et al. (1993b) \\
\hline 1175 & & & Neolebouria antarctica & Lepidonotothen squamifrons & $\begin{array}{l}\text { South Georgia (Shelf \& } \\
\text { Shag Rocks) }\end{array}$ & Zdzitowiecki et al. (1993b) \\
\hline 1176 & & & Neolebouria antarctica & Lepidonotothen squamifrons & South Shetland Islands & Palm et al. (2007) \\
\hline 1177 & & & Neolebouria antarctica & Lindbergichthys nudifrons & $\begin{array}{l}\text { South Shetland Islands } \\
\text { (Admiralty Bay) }\end{array}$ & Zdzitowiecki et al. (1993b) \\
\hline 1178 & DIGENEA & & Neolebouria antarctica & Lindbergichthys nudifrons & King George Island & Zdzitowiecki et al. (1993b) \\
\hline 1179 & & & Neolebouria antarctica & Lindbergichthys nudifrons & $\begin{array}{l}\text { Elephant Island (Joinville } \\
\text { Shelf) }\end{array}$ & Zdzitowiecki et al. (1993b) \\
\hline 1180 & & & Neolebouria antarctica & Lindbergichthys nudifrons & $\begin{array}{l}\text { South Georgia (Shelf \& } \\
\text { Shag Rocks) }\end{array}$ & Zdzitowiecki et al. (1993b) \\
\hline 1181 & & & Neolebouria antarctica & Lindbergichthys nudifrons & Vernadsky Station & $\begin{array}{l}\text { Laskowski \& Zdzitowiecki } \\
\text { (2005) }\end{array}$ \\
\hline 1182 & & & Neolebouria antarctica & Lindbergichthys nudifrons & South Shetland Islands & Palm et al. (2007) \\
\hline 1183 & & & Neolebouria antarctica & Neopagetopsis ionah & South Shetland Islands & Zdzitowiecki et al. (1993b) \\
\hline
\end{tabular}




\begin{tabular}{|c|c|c|c|c|c|c|}
\hline Index & Phylum & Family & Species & Host & Location & References \\
\hline & & & & & (Admiralty Bay) & \\
\hline 1184 & & & Neolebouria antarctica & Neopagetopsis ionah & King George Island & Zdzitowiecki et al. (1993b) \\
\hline 1185 & & & Neolebouria antarctica & Neopagetopsis ionah & $\begin{array}{l}\text { Elephant Island (Joinville } \\
\text { Shelf) }\end{array}$ & Zdzitowiecki et al. (1993b) \\
\hline 1186 & & & Neolebouria antarctica & Neopagetopsis ionah & $\begin{array}{c}\text { South Georgia (Shelf \& } \\
\text { Shag Rocks) }\end{array}$ & Zdzitowiecki et al. (1993b) \\
\hline 1187 & & & Neolebouria antarctica & Neopagetopsis ionah & South Shetland Islands & Zdzitowiecki (2002a) \\
\hline 1188 & & & Neolebouria antarctica & Notothenia coriiceps & $\begin{array}{l}\text { South Shetland Islands } \\
\text { (Admiralty Bay) }\end{array}$ & Zdzitowiecki et al. (1993b) \\
\hline 1189 & & & Neolebouria antarctica & Notothenia coriiceps & King George Island & Zdzitowiecki et al. (1993b) \\
\hline 1190 & & & Neolebouria antarctica & Notothenia coriiceps & $\begin{array}{l}\text { Elephant Island (Joinville } \\
\text { Shelf) }\end{array}$ & Zdzitowiecki et al. (1993b) \\
\hline 1191 & & & Neolebouria antarctica & Notothenia coriiceps & $\begin{array}{c}\text { South Georgia (Shelf \& } \\
\text { Shag Rocks) }\end{array}$ & Zdzitowiecki et al. (1993b) \\
\hline 1192 & & & Neolebouria antarctica & Notothenia coriiceps & Admiralty Bay & $\begin{array}{c}\text { Zdzitowiecki \& Laskowski } \\
\text { (2004) }\end{array}$ \\
\hline 1193 & & & Neolebouria antarctica & Notothenia coriiceps & Vernadsky Station & $\begin{array}{c}\text { Zdzitowiecki \& Laskowski } \\
\text { (2004) }\end{array}$ \\
\hline 1194 & & & Neolebouria antarctica & Notothenia rossii & South Georgia Island & Zdzitowiecki (1991) \\
\hline 1195 & & & Neolebouria antarctica & Notothenia rossii & $\begin{array}{l}\text { South Shetland Islands } \\
\text { (Admiralty Bay) }\end{array}$ & Zdzitowiecki et al. (1993b) \\
\hline 1196 & & & Neolebouria antarctica & Notothenia rossii & King George Island & Zdzitowiecki et al. (1993b) \\
\hline 1197 & & & Neolebouria antarctica & Notothenia rossii & $\begin{array}{l}\text { Elephant Island (Joinville } \\
\text { Shelf) }\end{array}$ & Zdzitowiecki et al. (1993b) \\
\hline 1198 & & & Neolebouria antarctica & Notothenia rossii & $\begin{array}{l}\text { South Georgia (Shelf \& } \\
\text { Shag Rocks) }\end{array}$ & Zdzitowiecki et al. (1993b) \\
\hline 1199 & & & Neolebouria antarctica & Nototheniops larseni & South Georgia Island & Zdzitowiecki (1991) \\
\hline 1200 & \multirow{8}{*}{ DIGENEA } & Opecoelidae & Neolebouria antarctica & Nototheniops larseni & $\begin{array}{l}\text { South Shetland Islands } \\
\text { (Admiralty Bay) }\end{array}$ & Zdzitowiecki et al. (1993b) \\
\hline 1201 & & & Neolebouria antarctica & Nototheniops larseni & King George Island & Zdzitowiecki et al. (1993b) \\
\hline 1202 & & & Neolebouria antarctica & Nototheniops larseni & $\begin{array}{l}\text { Elephant Island (Joinville } \\
\text { Shelf) }\end{array}$ & Zdzitowiecki et al. (1993b) \\
\hline 1203 & & & Neolebouria antarctica & Nototheniops larseni & $\begin{array}{l}\text { South Georgia (Shelf \& } \\
\text { Shag Rocks) }\end{array}$ & Zdzitowiecki et al. (1993b) \\
\hline 1204 & & & Neolebouria antarctica & Nototheniops larseni & South Shetland Islands & Palm et al. (2007) \\
\hline 1205 & & & Neolebouria antarctica & Parachaenichthys charcoti & South Shetland Islands & Zdzitowiecki (1991) \\
\hline 1206 & & & Neolebouria antarctica & Parachaenichthys charcoti & $\begin{array}{l}\text { South Shetland Islands } \\
\text { (Admiralty Bay) }\end{array}$ & Zdzitowiecki et al. (1993b) \\
\hline 1207 & & & Neolebouria antarctica & Parachaenichthys charcoti & King George Island & Zdzitowiecki et al. (1993b) \\
\hline
\end{tabular}




\begin{tabular}{|c|c|c|c|c|c|c|}
\hline Index & Phylum & Family & Species & Host & Location & References \\
\hline 1208 & & & Neolebouria antarctica & Parachaenichthys charcoti & $\begin{array}{l}\text { Elephant Island (Joinville } \\
\text { Shelf) }\end{array}$ & Zdzitowiecki et al. (1993b) \\
\hline 1209 & & & Neolebouria antarctica & Parachaenichthys charcoti & $\begin{array}{c}\text { South Georgia (Shelf \& } \\
\text { Shag Rocks) }\end{array}$ & Zdzitowiecki et al. (1993b) \\
\hline 1210 & & & Neolebouria antarctica & Parachaenichthys charcoti & Admiralty Bay & Zdzitowiecki (2001a) \\
\hline 1211 & & & Neolebouria antarctica & Parachaenichthys charcoti & $\begin{array}{l}\text { Off the South Shetland } \\
\text { Islands }\end{array}$ & Zdzitowiecki (2001a) \\
\hline 1212 & & & Neolebouria antarctica & Parachaenichthys charcoti & Bransfield Strait & Zdzitowiecki (2001a) \\
\hline 1213 & & & Neolebouria antarctica & Parachaenichthys georgianus & South Georgia Island & Zdzitowiecki (1979) \\
\hline 1214 & & & Neolebouria antarctica & Parachaenichthys georgianus & South Shetland Islands & Zdzitowiecki (1991) \\
\hline 1215 & & & Neolebouria antarctica & Parachaenichthys georgianus & South Georgia Island & Zdzitowiecki (1991) \\
\hline 1216 & & & Neolebouria antarctica & Parachaenichthys georgianus & $\begin{array}{l}\text { South Shetland Islands } \\
\text { (Admiralty Bay) }\end{array}$ & Zdzitowiecki et al. (1993b) \\
\hline 1217 & & & Neolebouria antarctica & Parachaenichthys georgianus & King George Island & Zdzitowiecki et al. (1993b) \\
\hline 1218 & & & Neolebouria antarctica & Parachaenichthys georgianus & $\begin{array}{l}\text { Elephant Island (Joinville } \\
\text { Shelf) }\end{array}$ & Zdzitowiecki et al. (1993b) \\
\hline 1219 & & & Neolebouria antarctica & Parachaenichthys georgianus & $\begin{array}{l}\text { South Georgia (Shelf \& } \\
\text { Shag Rocks) }\end{array}$ & Zdzitowiecki et al. (1993b) \\
\hline 1220 & & & Neolebouria antarctica & Paraliparis meganchus & $\begin{array}{l}\text { South Shetland Islands } \\
\text { (Admiralty Bay) }\end{array}$ & Zdzitowiecki et al. (1993b) \\
\hline 1221 & & & Neolebouria antarctica & Paraliparis meganchus & King George Island & Zdzitowiecki et al. (1993b) \\
\hline 1222 & & & Neolebouria antarctica & Paraliparis meganchus & $\begin{array}{l}\text { Elephant Island (Joinville } \\
\text { Shelf) }\end{array}$ & Zdzitowiecki et al. (1993b) \\
\hline 1223 & & & Neolebouria antarctica & Paraliparis meganchus & $\begin{array}{l}\text { South Georgia (Shelf \& } \\
\text { Shag Rocks) }\end{array}$ & Zdzitowiecki et al. (1993b) \\
\hline 1224 & & & Neolebouria antarctica & Pseudochaenichthys georgianus & South Georgia Island & Zdzitowiecki (1991) \\
\hline 1225 & & & Neolebouria antarctica & Pseudochaenichthys georgianus & $\begin{array}{l}\text { South Shetland Islands } \\
\text { (Admiralty Bay) }\end{array}$ & Zdzitowiecki et al. (1993b) \\
\hline 1226 & & & Neolebouria antarctica & Pseudochaenichthys georgianus & King George Island & Zdzitowiecki et al. (1993b) \\
\hline 1227 & \multirow{6}{*}{ DIGENEA } & Opecoelidae & Neolebouria antarctica & Pseudochaenichthys georgianus & $\begin{array}{l}\text { Elephant Island (Joinville } \\
\text { Shelf) }\end{array}$ & Zdzitowiecki et al. (1993b) \\
\hline 1228 & & & Neolebouria antarctica & Pseudochaenichthys georgianus & $\begin{array}{l}\text { South Georgia (Shelf \& } \\
\text { Shag Rocks) }\end{array}$ & Zdzitowiecki et al. (1993b) \\
\hline 1229 & & & Neolebouria antarctica & Psilodraco breviceps & South Georgia Island & Zdzitowiecki (1991) \\
\hline 1230 & & & Neolebouria antarctica & Psilodraco breviceps & $\begin{array}{l}\text { South Shetland Islands } \\
\text { (Admiralty Bay) }\end{array}$ & Zdzitowiecki et al. (1993b) \\
\hline 1231 & & & Neolebouria antarctica & Psilodraco breviceps & King George Island & Zdzitowiecki et al. (1993b) \\
\hline 1232 & & & Neolebouria antarctica & Psilodraco breviceps & $\begin{array}{c}\text { Elephant Island (Joinville } \\
\text { Shelf) }\end{array}$ & Zdzitowiecki et al. (1993b) \\
\hline
\end{tabular}




\begin{tabular}{|c|c|c|c|c|c|c|}
\hline Index & Phylum & Family & Species & Host & Location & References \\
\hline 1233 & & & Neolebouria antarctica & Psilodraco breviceps & $\begin{array}{c}\text { South Georgia (Shelf \& } \\
\text { Shag Rocks) }\end{array}$ & Zdzitowiecki et al. (1993b) \\
\hline 1234 & & & Neolebouria antarctica & Trematomus bernacchii & Vernadsky Station & $\begin{array}{l}\text { Laskowski \& Zdzitowiecki } \\
\text { (2005) }\end{array}$ \\
\hline 1235 & & & Neolebouria antarctica & Trematomus eulepidotus & South Shetland Islands & Zdzitowiecki (1991) \\
\hline 1236 & & & Neolebouria antarctica & Trematomus eulepidotus & $\begin{array}{l}\text { South Shetland Islands } \\
\text { (Admiralty Bay) }\end{array}$ & Zdzitowiecki et al. (1993b) \\
\hline 1237 & & & Neolebouria antarctica & Trematomus eulepidotus & King George Island & Zdzitowiecki et al. (1993b) \\
\hline 1238 & & & Neolebouria antarctica & Trematomus eulepidotus & $\begin{array}{l}\text { Elephant Island (Joinville } \\
\text { Shelf) }\end{array}$ & Zdzitowiecki et al. (1993b) \\
\hline 1239 & & & Neolebouria antarctica & Trematomus eulepidotus & $\begin{array}{l}\text { South Georgia (Shelf \& } \\
\text { Shag Rocks) }\end{array}$ & Zdzitowiecki et al. (1993b) \\
\hline 1240 & & & Neolebouria antarctica & Trematomus eulepidotus & South Shetland Islands & Palm et al. (2007) \\
\hline 1241 & & & Neolebouria antarctica & Trematomus hansoni & $\begin{array}{l}\text { South Shetland Islands } \\
\text { (Admiralty Bay) }\end{array}$ & Zdzitowiecki et al. (1993b) \\
\hline 1242 & & & Neolebouria antarctica & Trematomus hansoni & King George Island & Zdzitowiecki et al. (1993b) \\
\hline 1243 & & & Neolebouria antarctica & Trematomus hansoni & $\begin{array}{l}\text { Elephant Island (Joinville } \\
\text { Shelf) }\end{array}$ & Zdzitowiecki et al. (1993b) \\
\hline 1244 & & & Neolebouria antarctica & Trematomus hansoni & $\begin{array}{l}\text { South Georgia (Shelf \& } \\
\text { Shag Rocks) }\end{array}$ & Zdzitowiecki et al. (1993b) \\
\hline 1245 & & & Neolebouria antarctica & Trematomus newnesi & $\begin{array}{l}\text { South Shetland Islands } \\
\text { (Admiralty Bay) }\end{array}$ & Zdzitowiecki et al. (1993b) \\
\hline 1246 & & & Neolebouria antarctica & Trematomus newnesi & King George Island & Zdzitowiecki et al. (1993b) \\
\hline 1247 & & & Neolebouria antarctica & Trematomus newnesi & $\begin{array}{l}\text { Elephant Island (Joinville } \\
\text { Shelf) }\end{array}$ & Zdzitowiecki et al. (1993b) \\
\hline 1248 & & & Neolebouria antarctica & Trematomus newnesi & $\begin{array}{l}\text { South Georgia (Shelf \& } \\
\text { Shag Rocks) }\end{array}$ & Zdzitowiecki et al. (1993b) \\
\hline 1249 & & & Neolebouria antarctica & Trematomus newnesi & Admiralty Bay & $\begin{array}{c}\text { Laskowski \& Zdzitowiecki } \\
\text { (2005) }\end{array}$ \\
\hline 1250 & & & Neolebouria antarctica & Trematomus newnesi & Vernadsky Station & $\begin{array}{c}\text { Laskowski \& Zdzitowiecki } \\
\text { (2005) }\end{array}$ \\
\hline 1251 & & & Neolebouria antarctica & HOST NAME NOT MENTIONED & Glacial Subregion & Lyadov (1985) \\
\hline 1252 & & & Neolebouria terranovaensis & blank & blank & $\begin{array}{l}\text { Zdzitowiecki, Pisano \& Vacch } \\
\text { (1993) }\end{array}$ \\
\hline 1253 & & & Neolebouria terranovaensis & Paraliparis antarcticus & Weddell Sea & Zdzitowiecki (2003) \\
\hline 1254 & \multirow{4}{*}{ DIGENEA } & Opecoelidae & Neolebouria terranovaensis & Chionodraco hamatus & $\begin{array}{c}\text { Ross Sea (Terra Nova } \\
\text { Bay) }\end{array}$ & Zdzitowiecki et al. (1993b) \\
\hline 1255 & & & Neolebouria terranovaensis & Chionodraco hamatus & Adelie Land & Zdzitowiecki et.al. (1998) \\
\hline 1256 & & & Neolebouria terranovaensis & Chionodraco hamatus & Adelie Land & Zdzitowiecki (2001b) \\
\hline 1257 & & & Neolebouria terranovaensis & Chionodraco hamatus & Ross Sea & Zdzitowiecki (2002a) \\
\hline
\end{tabular}




\begin{tabular}{|c|c|c|c|c|c|c|}
\hline Index & Phylum & Family & Species & Host & Location & References \\
\hline 1258 & & & Neolebouria terranovaensis & Chionodraco hamatus & Adelie Land & Zdzitowiecki (2002a) \\
\hline 1259 & & & Neolebouria terranovaensis & Cygnodraco mawsoni & $\begin{array}{c}\text { Ross Sea (Terra Nova } \\
\text { Bay) }\end{array}$ & Zdzitowiecki et al. (1993b) \\
\hline 1260 & & & Neolebouria terranovaensis & Cygnodraco mawsoni & Ross Sea & Laskowski et.al. (2005) \\
\hline 1261 & & & Neolebouria terranovaensis & Gymnodraco acuticeps & Ross Sea & Laskowski et.al. (2005) \\
\hline 1262 & & & Neolebouria terranovaensis & Notothenia coriiceps & Adelie Land & Zdzitowiecki et.al. (1998) \\
\hline 1263 & & & Neolebouria terranovaensis & Notothenia coriiceps & Adelie Land & Zdzitowiecki (2001b) \\
\hline 1264 & & & Neolebouria terranovaensis & Paraliparis antarcticus & Weddell Sea & Zdzitowiecki (1997a) \\
\hline 1265 & & & Neolebouria terranovaensis & Pogonophryne scotti & $\begin{array}{c}\text { Ross Sea (Terra Nova } \\
\text { Bay) }\end{array}$ & Zdzitowiecki et al. (1993b) \\
\hline 1266 & & & Neolebouria terranovaensis & Trematomus hansoni & Adelie Land & Zdzitowiecki et.al. (1998) \\
\hline 1267 & & & Neolebouria terranovaensis & Trematomus newnesi & $\begin{array}{l}\text { Adelie Land (Eastern } \\
\text { Antarctica) }\end{array}$ & Laskowski et.al. (2007) \\
\hline 1268 & & & Neolebouria terranovaensis & Trematomus T. pennellii & $\begin{array}{c}\text { Ross Sea (Terra Nova } \\
\text { Bay) }\end{array}$ & Zdzitowiecki et al. (1993b) \\
\hline 1269 & & & Opecoelus scorpenidicola & blank & blank & Prudhoe \& Bray (1973) \\
\hline 1270 & & & Opecoelus scorpenidicola & Helicolenus percoides & $42^{\circ} 40^{\prime} \mathrm{S} 148^{\circ} 27^{\prime} 30^{\prime \prime} \mathrm{E}$ & Prudhoe \& Bray (1973) \\
\hline 1271 & & & Opecoelus scorpenidicola & Helicolenus percoides & Off Maria, Tasmania & Prudhoe \& Bray (1973) \\
\hline 1272 & & & Opecoelus scorpenidicola & Lepidoperca tasmanica & $42^{\circ} 40^{\prime} \mathrm{S} 148^{\circ} 27^{\prime} 30^{\prime \prime} \mathrm{E}$ & Prudhoe \& Bray (1973) \\
\hline 1273 & & & Opecoelus scorpenidicola & Lepidoperca tasmanica & Off Maria, Tasmania & Prudhoe \& Bray (1973) \\
\hline 1274 & & & Opecoelus scorpenidicola & Scorpaena cruenta & $42^{\circ} 40^{\prime} \mathrm{S} 148^{\circ} 27^{\prime} 30^{\prime \prime} \mathrm{E}$ & Prudhoe \& Bray (1973) \\
\hline 1275 & & & Opecoelus scorpenidicola & Scorpaena cruenta & Off Maria, Tasmania & Prudhoe \& Bray (1973) \\
\hline 1276 & & & Opecoelus scorpenidicola & HOST NAME NOT MENTIONED & South Australia & Lyadov (1985) \\
\hline 1277 & & & Opegaster synodi & blank & blank & $\begin{array}{c}\text { Manter }(1947)=\text { Neolebouria } \\
\text { antarctica }\end{array}$ \\
\hline 1278 & & & Opegaster synodi & blank & blank & Szidat \& Graefe (1967) \\
\hline 1279 & & & Opegaster synodi & Notothenia coriiceps & $\begin{array}{c}\text { Bhaia Scotia La Isla } \\
\text { Laurie (Orcadas del sur, } \\
\text { Argentina) }\end{array}$ & Szidat (1965) \\
\hline 1280 & & & Opegaster synodi & Notothenia coriiceps & Isle Laurie & Szidat (1965) \\
\hline 1281 & & & Opegaster synodi & Notothenia coriiceps & Orcadas Del Sur & Szidat (1965) \\
\hline 1282 & DIGENEA & Opecoelidae & Pseudopecoelus pyriformis & blank & blank & Prudhoe \& Bray (1973) \\
\hline
\end{tabular}




\begin{tabular}{|c|c|c|c|c|c|c|}
\hline Index & Phylum & Family & Species & Host & Location & References \\
\hline 1283 & & & Pseudopecoelus pyriformis & Callanthias allporti & $42^{\circ} 40^{\prime} \mathrm{S} 148^{\circ} 27^{\prime} 30^{\prime \prime} \mathrm{E}$ & Prudhoe \& Bray (1973) \\
\hline 1284 & & & Pseudopecoelus pyriformis & Callanthias allporti & Off Maria, Tasmania & Prudhoe \& Bray (1973) \\
\hline 1285 & & & Pseudopecoelus pyriformis & HOST NAME NOT MENTIONED & South Australia & Lyadov (1985) \\
\hline 1286 & & & Stenakron sp. indet. & blank & blank & Stafford (1904) \\
\hline 1287 & & & Stenakron sp. indet. & Aconichthys harrisoni & A.A.E. & Prudhoe \& Bray (1973) \\
\hline 1288 & & & Stenakron sp. indet. & Aconichthys harrisoni & $65^{\circ} 6^{\prime} \mathrm{S} 96^{\circ} 14^{\prime} \mathrm{E}$ & Prudhoe \& Bray (1973) \\
\hline 1289 & & & Stenakron sp. indet. & Paraliparis wildi & A.A.E. & Prudhoe \& Bray (1973) \\
\hline 1290 & & & Stenakron sp. indet. & Paraliparis wildi & $65^{\circ} 6^{\prime} \mathrm{S} 96^{\circ} 14^{\prime} \mathrm{E}$ & Prudhoe \& Bray (1973) \\
\hline 1291 & & & Stenakron sp. & blank & blank & Stafford (1904) \\
\hline 1292 & & & Stenakron sp. & Dissostichus eleginoides & Prince Edward Island & Brickle et al. (2005) \\
\hline 1293 & & & Stenakron sp. & HOST NAME NOT MENTIONED & Glacial Subregion & Lyadov (1985) \\
\hline 1294 & & & Stenakron glacialis & blank & blank & Zdzitowiecki (1989) \\
\hline 1295 & & & Stenakron glacialis & Artedidroconidae & Weddell Sea & Zdzitowiecki (2002c) \\
\hline 1296 & & & Stenakron glacialis & Pogonophryne marmorata & Weddell Sea & Zdzitowiecki (1997a) \\
\hline 1297 & & & Stenakron glacialis & Pogonophryne marmorata & Weddell Sea & Zdzitowiecki (2002c) \\
\hline 1298 & & & Stenakron glacialis & Racovitzia glacialis & Weddell Sea & Zdzitowiecki (1997a) \\
\hline 1299 & & & Stenakron glacialis & Racovitzia glacialis & Weddell Sea & Zdzitowiecki (2002d) \\
\hline 1300 & & & Stenakron Kerguelen Subregionse & blank & blank & Prudhoe \& Bray (1973) \\
\hline 1301 & & & Stenakron Kerguelen Subregionse & Champsocephalus gunnari & $49^{\circ} 28^{\prime} \mathrm{S} 70^{\circ} 33^{\prime} \mathrm{E}$ & Prudhoe \& Bray (1973) \\
\hline 1302 & & & Stenakron Kerguelen Subregionse & Champsocephalus gunnari & $\begin{array}{l}\text { Off Entrance To Royal } \\
\text { Sound (Kerguelen } \\
\text { Subregion) }\end{array}$ & Prudhoe \& Bray (1973) \\
\hline 1303 & & & Stenakron Kerguelen Subregionse & Notothenia cyanobrancha & $\begin{array}{l}\text { From various stations at } \\
\text { Kerguelen Subregion }\end{array}$ & Prudhoe \& Bray (1973) \\
\hline 1304 & & & Stenakron Kerguelen Subregionse & Notothenia Kerguelen Subregionse & Heard Island & Prudhoe \& Bray (1973) \\
\hline 1305 & & & Stenakron Kerguelen Subregionse & Zanclorhynchus spinifer & $54^{\circ} 42^{\prime} 30^{\prime \prime} \mathrm{S} 158^{\circ} 54^{\prime} 30^{\prime \prime} \mathrm{E}$ & Prudhoe \& Bray (1973) \\
\hline 1306 & & & Stenakron Kerguelen Subregionse & Zanclorhynchus spinifer & $\begin{array}{l}\text { Off Lusitania Bay, } \\
\text { Macquarie Island }\end{array}$ & Prudhoe \& Bray (1973) \\
\hline 1307 & & & Stenakron Kerguelen Subregionse & HOST NAME NOT MENTIONED & Kerguelen Subregion & Lyadov (1985) \\
\hline 1308 & & Sanguinicolidae & Aporocotyle nototheniae & Notothenia rossii & Ob Bank & Parukhin (1986) \\
\hline
\end{tabular}




\begin{tabular}{|c|c|c|c|c|c|c|}
\hline Index & Phylum & Family & Species & Host & Location & References \\
\hline 1309 & \multirow{4}{*}{ DIGENEA } & Syncoeliidae & Steganodermatiodes kergeleni & blank & blank & Parukhin \& Lyadov (1979) \\
\hline 1310 & & Syncoeliidae & Steganodermatiodes kergeleni & HOST NAME NOT MENTIONED & Kerguelen Subregion & Lyadov (1985) \\
\hline 1311 & & & Whitegonimus ozoufae & blank & blank & $\begin{array}{l}\text { Jeżewski, Zdzitowiecki \& } \\
\text { Laskowski (2009) }\end{array}$ \\
\hline 1312 & & & Whitegonimus ozoufae & Patagonotothen tesellata & $\begin{array}{c}\text { Harbor of Ushuaia In The } \\
\text { Beagle Channel }\end{array}$ & Jeżewski et al. (2009) \\
\hline 1313 & \multirow{22}{*}{ CESTODA } & blank & $\begin{array}{c}\text { Bilocular trilocular cercoids } \\
\text { plerocercoids }\end{array}$ & Parachaenichthys charcoti & $\begin{array}{l}\text { Admiralty Bay Off The } \\
\text { South Shetland Islands }\end{array}$ & Zdzitowiecki (2001a) \\
\hline 1314 & & & Bilocular metacestode & Trematomus newnesi & $\begin{array}{l}\text { Adelie Land (Eastern } \\
\text { Antarctica) }\end{array}$ & Laskowski et.al. (2007) \\
\hline 1315 & & & Bilocular cercoids & Cygnodraco mawsoni & Ross Sea & Laskowski et.al. (2005) \\
\hline 1316 & & & Bilocular cercoids & Cygnodraco mawsoni & South Shetland Islands & Laskowski et.al. (2005) \\
\hline 1317 & & & Bilocular cercoids & Gymnodraco acuticeps & South Shetland Islands & Laskowski et.al. (2005) \\
\hline 1318 & & & Bilocular cercoids & Gymnodraco acuticeps & Ross Sea & Laskowski et.al. (2005) \\
\hline 1319 & & & Cercoid bilocular & Harpagifer georgianus & Admiralty Bay & Zdzitowiecki \& Zadrozny (1999) \\
\hline 1320 & & & Cercoid bilocular & Parachaenichthys charcoti & Admiralty Bay & Zdzitowiecki (2001a) \\
\hline 1321 & & & Cercoid bilocular & Parachaenichthys charcoti & Bransfield Strait & Zdzitowiecki (2001a) \\
\hline 1322 & & & Cercoid IV (tetraphyllidean bilocular) & Notothenia coriiceps & Signy Island & Zdzitowiecki et al. (1997) \\
\hline 1323 & & & Cercoid IV (tetraphyllidean bilocular) & Gobionotothen gibberifrons & Signy Island & Zdzitowiecki et al. (1997) \\
\hline 1324 & & & Cercoid IV (tetraphyllidean bilocular) & Chaenocephalus aceratus & Signy Island & Zdzitowiecki et al. (1997) \\
\hline 1325 & & & Cercoid IV (tetraphyllidean bilocular) & Notothenia coriiceps & Orkny Islands & Zdzitowiecki et al. (1997) \\
\hline 1326 & & & Cercoid IV (tetraphyllidean bilocular) & Gobionotothen gibberifrons & Orkny Islands & Zdzitowiecki et al. (1997) \\
\hline 1327 & & & Cercoid IV (tetraphyllidean bilocular) & Chaenocephalus aceratus & Orkny Islands & Zdzitowiecki et al. (1997) \\
\hline 1328 & & & Cercoid monolocular (tetraphyllidean) & Harpagifer georgianus & Admiralty Bay & Zdzitowiecki \& Zadrozny (1999) \\
\hline 1329 & & & Cercoid trilocular (tetraphyllidean) & Harpagifer georgianus & Admiralty Bay & Zdzitowiecki \& Zadrozny (1999) \\
\hline 1330 & & & $\begin{array}{l}\text { Cercoid with bilocular bothridia } \\
\text { (probably Pseudanthobothrium sp.) }\end{array}$ & Chionodraco hamatus & $\begin{array}{l}\text { Terra Nova Bay (Ross } \\
\text { Sea) At Italian Station }\end{array}$ & Wojciechowska et.al. (1994) \\
\hline 1331 & & & $\begin{array}{l}\text { Cercoid with bilocular bothridia } \\
\text { (probably Pseudanthobothrium sp.) }\end{array}$ & Cryodraco antarcticus & $\begin{array}{l}\text { Terra Nova Bay (Ross } \\
\text { Sea) At Italian Station }\end{array}$ & Wojciechowska et.al. (1994) \\
\hline 1332 & & & $\begin{array}{l}\text { Cercoid with bilocular bothridia } \\
\text { (probably Pseudanthobothrium sp.) }\end{array}$ & Cygnodraco mawsoni & $\begin{array}{l}\text { Terra Nova Bay (Ross } \\
\text { Sea) At Italian Station }\end{array}$ & Wojciechowska et.al. (1994) \\
\hline 1333 & & & $\begin{array}{l}\text { Cercoid with bilocular bothridia } \\
\text { (probably Pseudanthobothrium sp.) }\end{array}$ & Pogonophryne scotti & $\begin{array}{l}\text { Terra Nova Bay (Ross } \\
\text { Sea) At Italian Station }\end{array}$ & Wojciechowska et.al. (1994) \\
\hline 1334 & & & $\begin{array}{l}\text { Cercoid with bilocular bothridia } \\
\text { (probably Pseudanthobothrium sp.) }\end{array}$ & Trematomus centronotus & $\begin{array}{l}\text { Terra Nova Bay (Ross } \\
\text { Sea) At Italian Station }\end{array}$ & Wojciechowska et.al. (1994) \\
\hline
\end{tabular}




\begin{tabular}{|c|c|c|c|c|c|c|}
\hline Index & Phylum & Family & Species & Host & Location & References \\
\hline 1335 & & & $\begin{array}{l}\text { Cercoid with trilocular bothridia } \\
\text { (probably Onchobothrium sp.) }\end{array}$ & Cryodraco antarcticus & $\begin{array}{l}\text { Terra Nova Bay (Ross } \\
\text { Sea) At Italian Station }\end{array}$ & Wojciechowska et.al. (1994) \\
\hline 1336 & & & $\begin{array}{l}\text { Cercoid with trilocular bothridia } \\
\text { (probably Onchobothrium sp.) }\end{array}$ & Pogonophryne scotti & $\begin{array}{l}\text { Terra Nova Bay (Ross } \\
\text { Sea) At Italian Station }\end{array}$ & Wojciechowska et.al. (1994) \\
\hline 1337 & & & $\begin{array}{l}\text { Cerciod with subcylindrical bothria } \\
\text { (possibly Marsupobothrium sp.) }\end{array}$ & Cryodraco antarcticus & $\begin{array}{l}\text { Terra Nova Bay (Ross } \\
\text { Sea) At Italian Station }\end{array}$ & Wojciechowska et.al. (1994) \\
\hline 1338 & & blank & $\begin{array}{l}\text { Cerciod with subcylindrical bothria } \\
\text { (possibly Marsupobothrium sp.) }\end{array}$ & Pogonophryne scotti & $\begin{array}{l}\text { Terra Nova Bay (Ross } \\
\text { Sea) At Italian Station }\end{array}$ & Wojciechowska et.al. (1994) \\
\hline 1339 & & & $\begin{array}{l}\text { Cerciod with subcylindrical bothria } \\
\text { (possibly Marsupobothrium sp.) }\end{array}$ & Trematomus centronotus & $\begin{array}{l}\text { Terra Nova Bay (Ross } \\
\text { Sea) At Italian Station }\end{array}$ & Wojciechowska et.al. (1994) \\
\hline 1340 & & & Cercoid VI (tetraphyllidean trilocular) & Gobionotothen gibberifrons & Orkny Islands & Zdzitowiecki et al. (1997) \\
\hline 1341 & & & Cercoid VI (tetraphyllidean trilocular) & Chaenocephalus aceratus & Signy Island & Zdzitowiecki et al. (1997) \\
\hline 1342 & & & Cercoid VI (tetraphyllidean trilocular) & Gobionotothen gibberifrons & Orkny Islands & Zdzitowiecki et al. (1997) \\
\hline 1343 & & & Cercoid VI (tetraphyllidean trilocular) & Chaenocephalus aceratus & Signy Island & Zdzitowiecki et al. (1997) \\
\hline 1344 & & & Cercoid I monolocular & Champsocephalus gunnari & Admiralty Bay Shelves & Wojciechowska (1993a) \\
\hline 1345 & & & Cercoid I monolocular & Champsocephalus gunnari & Mesopelagic Zone & Wojciechowska (1993a) \\
\hline 1346 & & & Cercoid I monolocular & Champsocephalus gunnari & $\begin{array}{l}\text { At The South Shetland } \\
\text { Islands }\end{array}$ & Wojciechowska (1993a) \\
\hline 1347 & & & Cercoid I monolocular & Champsocephalus gunnari & Joinville Island & Wojciechowska (1993a) \\
\hline 1348 & & & Cercoid I monolocular & Chiondraco rastrospinosus & Admiralty Bay Shelves & Wojciechowska (1993a) \\
\hline 1349 & CESTODA & & Cercoid I monolocular & Chiondraco rastrospinosus & Mesopelagic Zone & Wojciechowska (1993a) \\
\hline 1350 & & & Cercoid I monolocular & Chiondraco rastrospinosus & $\begin{array}{l}\text { At The South Shetland } \\
\text { Islands }\end{array}$ & Wojciechowska (1993a) \\
\hline 1351 & & & Cercoid I monolocular & Chiondraco rastrospinosus & Joinville Island & Wojciechowska (1993a) \\
\hline 1352 & & & Cercoid I monolocular & Notothenia neglecta & Admiralty Bay Shelves & Wojciechowska (1993a) \\
\hline 1353 & & & Cercoid I monolocular & Notothenia neglecta & Mesopelagic Zone & Wojciechowska (1993a) \\
\hline 1354 & & & Cercoid I monolocular & Notothenia neglecta & $\begin{array}{l}\text { At The South Shetland } \\
\text { Islands }\end{array}$ & Wojciechowska (1993a) \\
\hline 1355 & & & Cercoid I monolocular & Notothenia neglecta & Joinville Island & Wojciechowska (1993a) \\
\hline 1356 & & & Cercoid I monolocular & Notothenia rossii & Admiralty Bay Shelves & Wojciechowska (1993a) \\
\hline 1357 & & & Cercoid I monolocular & Notothenia rossii & Mesopelagic Zone & Wojciechowska (1993a) \\
\hline 1358 & & & Cercoid I monolocular & Notothenia rossii & $\begin{array}{l}\text { At The South Shetland } \\
\text { Islands }\end{array}$ & Wojciechowska (1993a) \\
\hline 1359 & & & Cercoid I monolocular & Notothenia rossii & Joinville Island & Wojciechowska (1993a) \\
\hline 1360 & & & Cercoid I monolocular & Paradiplospinus antarcticus & Admiralty Bay Shelves & Wojciechowska (1993a) \\
\hline
\end{tabular}




\begin{tabular}{|c|c|c|c|c|c|c|}
\hline Index & Phylum & Family & Species & Host & Location & References \\
\hline 1361 & & & Cercoid I monolocular & Paradiplospinus antarcticus & Mesopelagic Zone & Wojciechowska (1993a) \\
\hline 1362 & & & Cercoid I monolocular & Paradiplospinus antarcticus & $\begin{array}{l}\text { At The South Shetland } \\
\text { Islands }\end{array}$ & Wojciechowska (1993a) \\
\hline 1363 & & & Cercoid I monolocular & Paradiplospinus antarcticus & Joinville Island & Wojciechowska (1993a) \\
\hline 1364 & & & Cercoid II monolocular & Champsocephalus gunnari & South Georgia (Shelves) & Wojciechowska (1993a) \\
\hline 1365 & & blank & Cercoid II monolocular & Parachaenichthys georgianus & South Georgia (Shelves) & Wojciechowska (1993a) \\
\hline 1366 & & & Cercoid III monolocular & Electrona carlsbergi & $\begin{array}{l}\text { Antarctic Convergence } \\
\text { Line }\end{array}$ & Wojciechowska (1993a) \\
\hline 1367 & & & Cercoid III monolocular & Paradiplospinus antarcticus & South Shetland Islands & Wojciechowska (1993a) \\
\hline 1368 & & & Cercoid IV bilocular & Chaenocephalus aceratus & South Shetland Islands & Wojciechowska (1993a) \\
\hline 1369 & & & Cercoid IV bilocular & Champsocephalus gunnari & South Shetland Islands & Wojciechowska (1993a) \\
\hline 1370 & & & Cercoid IV bilocular & Cryodraco antarcticus & South Shetland Islands & Wojciechowska (1993a) \\
\hline 1371 & & & Cercoid IV bilocular & Notothenia neglecta & South Shetland Islands & Wojciechowska (1993a) \\
\hline 1372 & & & Cercoid IV bilocular & Notothenia rossii & South Shetland Islands & Wojciechowska (1993a) \\
\hline 1373 & & & Cercoid IV bilocular & Nototheniops nudifrons & South Shetland Islands & Wojciechowska (1993a) \\
\hline 1374 & & & Cercoid IV bilocular & Parachaenichthys charcoti & South Shetland Islands & Wojciechowska (1993a) \\
\hline 1375 & & & Cerciod V bilocular & Champsocephalus gunnari & $\begin{array}{l}\text { Environs of South Georgia } \\
\text { (Open Sea Shelf) }\end{array}$ & Wojciechowska (1993a) \\
\hline 1376 & CESTODA & & Cerciod V bilocular & Muraenolepis microps & $\begin{array}{l}\text { Environs of South Georgia } \\
\text { (Open Sea Shelf) }\end{array}$ & Wojciechowska (1993a) \\
\hline 1377 & & & Cerciod V bilocular & Parachaenichthys charcoti & $\begin{array}{l}\text { Environs of South Georgia } \\
\text { (Open Sea Shelf) }\end{array}$ & Wojciechowska (1993a) \\
\hline 1378 & & & Cercoid VI trilocular & Chaenocephalus aceratus & Admiralty Bay & Wojciechowska (1993a) \\
\hline 1379 & & & Cercoid VI trilocular & Notothenia neglecta & Admiralty Bay & Wojciechowska (1993a) \\
\hline 1380 & & & Cercoid VI trilocular & Parachaenichthys charcoti & Admiralty Bay & Wojciechowska (1993a) \\
\hline 1381 & & & Cercoid VI trilocular & Chiondraco rastrospinosus & $\begin{array}{l}\text { South Shetland Islands } \\
\text { (Open Sea Shelf) }\end{array}$ & Wojciechowska (1993a) \\
\hline 1382 & & & Cercoid VI trilocular & Parachaenichthys georgianus & $\begin{array}{l}\text { South Shetland Islands } \\
\text { (Open Sea Shelf) }\end{array}$ & Wojciechowska (1993a) \\
\hline 1383 & & & $\begin{array}{l}\text { Cercoid VII with leaf-like bothria as } \\
\text { accessory succer }\end{array}$ & Champsocephalus gunnari & $\begin{array}{l}\text { South Georgia-Shelf At } \\
\text { Shag Rocks }\end{array}$ & Wojciechowska (1993a) \\
\hline 1384 & & & Cercoid VIII (lacking bothria) & Champsocephalus gunnari & South Georgia Island & Wojciechowska (1993a) \\
\hline 1385 & & & Cercoid VIII (lacking bothria) & Champsocephalus gunnari & Chumberland Bay & Wojciechowska (1993a) \\
\hline 1386 & & & Cercoid VIII (lacking bothria) & Harpagifer georgianus & South Georgia Island & Wojciechowska (1993a) \\
\hline
\end{tabular}




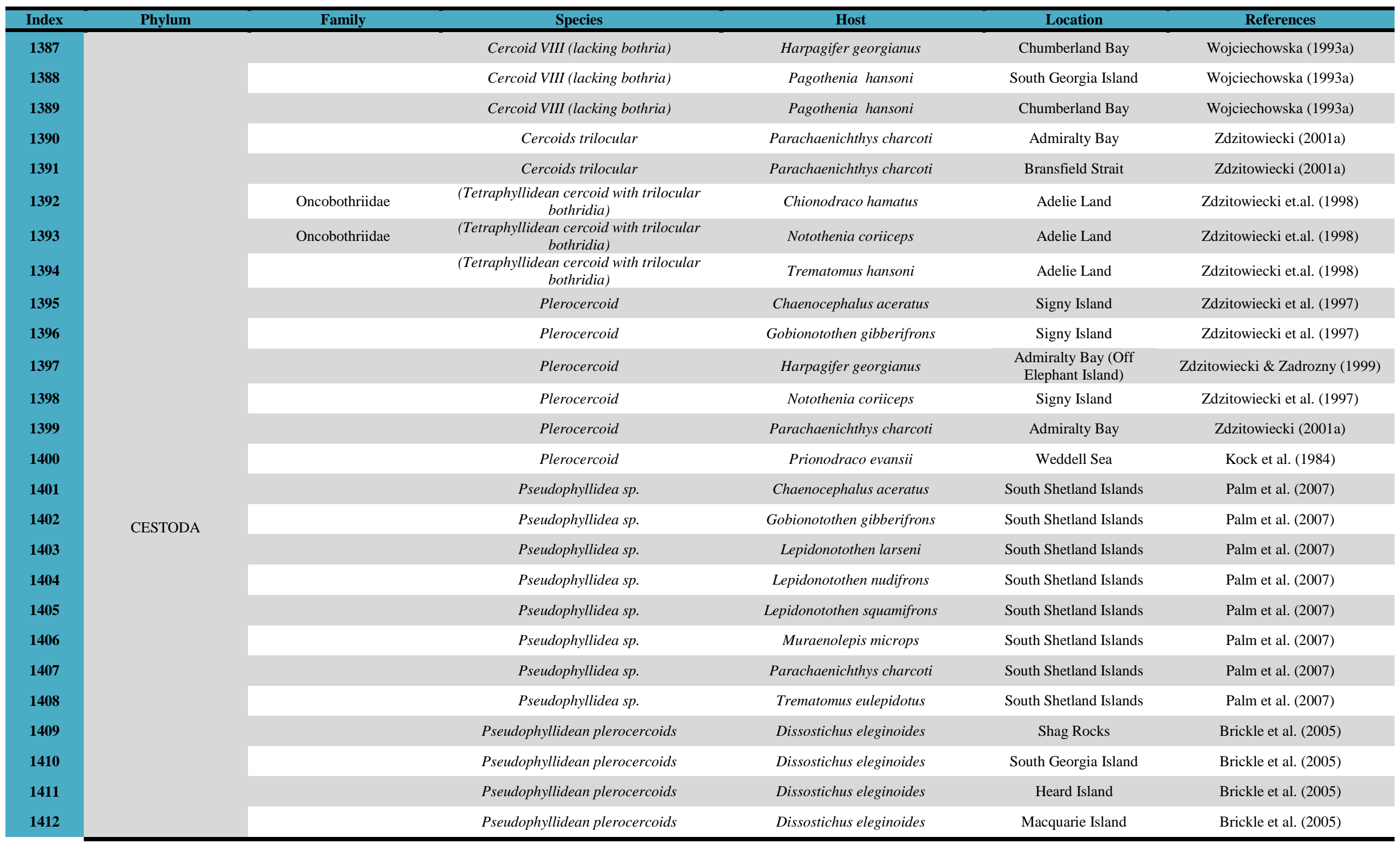




\begin{tabular}{|c|c|c|c|c|c|c|}
\hline Index & Phylum & Family & Species & Host & Location & References \\
\hline 1413 & & & Pseudophyllidean plerocercoids & Dissostichus eleginoides & Falkland Islands & Brickle et al. (2005) \\
\hline 1414 & & & Tetraphyllidae gen.sp. Larvae & Chaenichthys rhinoceratus & Kerguelen Subregion & Parukhin \& Lyadov (1981) \\
\hline 1415 & & & Tetraphyllidae gen.sp. Larvae & Chaenichthys rhinoceratus & Kerguelen Subregion & Lyadov et al. (1981) \\
\hline 1416 & & & Tetraphyllidae gen.sp. Larvae & Chaenichthys velifer & Kerguelen Subregion & Parukhin \& Lyadov (1981) \\
\hline 1417 & & & Tetraphyllidae gen.sp. Larvae & Chaenichthys velifer & Kerguelen Subregion & Lyadov et al. (1981) \\
\hline 1418 & & & Tetraphyllidae gen.sp. Larvae & Champsocephalus gunnari & Kerguelen Subregion & Parukhin \& Lyadov (1981) \\
\hline 1419 & & & Tetraphyllidae gen.sp. Larvae & Champsocephalus gunnari & Kerguelen Subregion & Lyadov et al. (1981) \\
\hline 1420 & & & Tetraphyllidae gen.sp. Larvae & Dissostichus eleginoides & Kerguelen Subregion & Parukhin \& Lyadov (1981) \\
\hline 1421 & & & Tetraphyllidae gen.sp. Larvae & Dissostichus eleginoides & Kerguelen Subregion & Parukhin \& Lyadov (1982) \\
\hline 1422 & \multirow{18}{*}{ CESTODA } & Oncobothriidae & Tetraphyllidae gen.sp. Larvae & Dissostichus eleginoides & Skif Bank & Parukhin \& Lyadov (1982) \\
\hline 1423 & & & Tetraphyllidae gen.sp. Larvae & Dissostichus eleginoides & Ob Bank & Parukhin \& Lyadov (1982) \\
\hline 1424 & & & Tetraphyllidae gen.sp. Larvae & Dissostichus eleginoides & Lena Bank & Parukhin \& Lyadov (1982) \\
\hline 1425 & & & Tetraphyllidae gen.sp. Larvae & Dissostichus eleginoides & Ob Bank & Parukhin (1986) \\
\hline 1426 & & & Tetraphyllidae gen.sp. Larvae & Dissostichus eleginoides & Lena Bank & Parukhin (1986) \\
\hline 1427 & & & Tetraphyllidae gen.sp. Larvae & Notothenia acuta & Kerguelen Subregion & Parukhin \& Lyadov (1981) \\
\hline 1428 & & & Tetraphyllidae gen.sp. Larvae & Notothenia acuta & Kerguelen Subregion & Parukhin \& Lyadov (1982) \\
\hline 1429 & & & Tetraphyllidae gen.sp. Larvae & Notothenia larseni & Lena Bank & Parukhin (1986) \\
\hline 1430 & & & Tetraphyllidae gen.sp. Larvae & Notothenia larseni & Ob Bank & Parukhin (1986) \\
\hline 1431 & & & Tetraphyllidae gen.sp. Larvae & Notothenia mizops & Kerguelen Subregion & Parukhin \& Lyadov (1981) \\
\hline 1432 & & & Tetraphyllidae gen.sp. Larvae & Notothenia mizops & Kerguelen Subregion & Parukhin \& Lyadov (1982) \\
\hline 1433 & & & Tetraphyllidae gen.sp. Larvae & Notothenia ramsayi & Folkland & Parukhin \& Lyadov (1981) \\
\hline 1434 & & & Tetraphyllidae gen.sp. Larvae & Notothenia ramsayi & Folkland & Parukhin \& Lyadov (1981) \\
\hline 1435 & & & Tetraphyllidae gen.sp. Larvae & Notothenia rossii & Kerguelen Subregion & Parukhin \& Lyadov (1981) \\
\hline 1436 & & & Tetraphyllidae gen.sp. Larvae & Notothenia rossii & Kerguelen Subregion & Parukhin \& Lyadov (1982) \\
\hline 1437 & & & Tetraphyllidae gen.sp. Larvae & Notothenia rossii & Kherd Island & Parukhin \& Lyadov (1982) \\
\hline 1438 & & & Tetraphyllidae gen.sp. Larvae & Notothenia rossii & Crozet Islands & Parukhin \& Lyadov (1982) \\
\hline 1439 & & & Tetraphyllidae gen.sp. Larvae & Notothenia rossii & Ob Bank & Parukhin \& Lyadov (1982) \\
\hline
\end{tabular}




\begin{tabular}{|c|c|c|c|c|c|c|}
\hline Index & Phylum & Family & Species & Host & Location & References \\
\hline 1440 & & & Tetraphyllidae gen.sp. Larvae & Notothenia rossii & Ob Bank & Parukhin (1986) \\
\hline 1441 & & & Tetraphyllidae gen.sp. Larvae & Notothenia squamifrons & Kerguelen Subregion & Parukhin \& Lyadov (1981) \\
\hline 1442 & & & Tetraphyllidae gen.sp. Larvae & Notothenia squamifrons & Kerguelen Subregion & Parukhin \& Lyadov (1982) \\
\hline 1443 & & & Tetraphyllidae gen.sp. Larvae & Notothenia squamifrons & Crozet Islands & Parukhin \& Lyadov (1982) \\
\hline 1444 & & & Tetraphyllidae gen.sp. Larvae & Notothenia squamifrons & Skif Bank & Parukhin \& Lyadov (1982) \\
\hline 1445 & & & Tetraphyllidae gen.sp. Larvae & Notothenia squamifrons & Ob Bank & Parukhin \& Lyadov (1982) \\
\hline 1446 & & & Tetraphyllidae gen.sp. Larvae & Notothenia squamifrons & Lena Bank & Parukhin \& Lyadov (1982) \\
\hline 1447 & & & Tetraphyllidae gen.sp. Larvae & Notothenia squamifrons & Lena Bank & Parukhin (1986) \\
\hline 1448 & & & Tetraphyllidae gen.sp. Larvae & Nototheniidae & Kerguelen Subregion & Parukhin \& Lyadov (1982) \\
\hline 1449 & & & Tetraphyllidae gen.sp. Larvae & HOST NAME NOT MENTIONED & Glacial Subregion & Lyadov (1985) \\
\hline 1450 & & & Tetraphyllidae gen.sp. Larvae & HOST NAME NOT MENTIONED & Kerguelen Subregion & Lyadov (1985) \\
\hline 1451 & \multirow{14}{*}{ CESTODA } & Oncobothriidae & Tetraphyllidae gen.sp. Larvae & HOST NAME NOT MENTIONED & Patagonian Shelf & Lyadov (1985) \\
\hline 1452 & & & Tetraphyllidae gen.sp. Larvae & HOST NAME NOT MENTIONED & South Australia & Lyadov (1985) \\
\hline 1453 & & & Tetraphyllidean cercoid bilocular & Lepidonotohen larseni & $\begin{array}{l}\text { Port Foster (Deception } \\
\text { Island) }\end{array}$ & Ruhl et al. (2003) \\
\hline 1454 & & & Tetraphyllidean cercoid bilocular & Trematomus bernacchii & $\begin{array}{l}\text { Port Foster (Deception } \\
\text { Island) }\end{array}$ & Ruhl et al. (2003) \\
\hline 1455 & & & Tetraphyllidean cercoid bilocular & Lepidonotohen larseni & $\begin{array}{l}\text { Port Foster (Deception } \\
\text { Island) }\end{array}$ & Ruhl et al. (2003) \\
\hline 1456 & & & Tetraphyllidean cercoid bilocular & Trematomus bernacchii & $\begin{array}{l}\text { Port Foster (Deception } \\
\text { Island) }\end{array}$ & Ruhl et al. (2003) \\
\hline 1457 & & & $\begin{array}{l}\text { Tetraphyllidean cercoid with bilocular } \\
\text { bothria }\end{array}$ & Chionodraco hamatus & Adelie Land & Zdzitowiecki (2001b) \\
\hline 1458 & & & $\begin{array}{l}\text { Tetraphyllidean cercoid with bilocular } \\
\text { bothria }\end{array}$ & Notothenia coriiceps & Adelie Land & Zdzitowiecki (2001b) \\
\hline 1459 & & & $\begin{array}{l}\text { Tetraphyllidean cercoid with bilocular } \\
\text { bothria }\end{array}$ & Trematomus hansoni & Adelie Land & Zdzitowiecki (2001b) \\
\hline 1460 & & & $\begin{array}{c}\text { Tetraphyllidean cercoid with trilocular } \\
\text { bothria }\end{array}$ & Chionodraco hamatus & Adelie Land & Zdzitowiecki (2001b) \\
\hline 1461 & & & $\begin{array}{l}\text { Tetraphyllidean cercoid with trilocular } \\
\text { bothria }\end{array}$ & Notothenia coriiceps & Adelie Land & Zdzitowiecki (2001b) \\
\hline 1462 & & & $\begin{array}{c}\text { Tetraphyllidean cercoid with trilocular } \\
\text { bothria }\end{array}$ & Trematomus hansoni & Adelie Land & Zdzitowiecki (2001b) \\
\hline 1463 & & & $\begin{array}{c}\text { Tetraphyllidean cercoids (bilocular } \\
\text { bothria) }\end{array}$ & Bathyraja eatoni & Heard Island Plateau & Wojciechowska et al. (1995) \\
\hline 1464 & & & $\begin{array}{c}\text { Tetraphyllidean cercoids (bilocular } \\
\text { bothria) }\end{array}$ & Champsocephalus gunnari & Heard Island Plateau & Wojciechowska et al. (1995) \\
\hline
\end{tabular}




\begin{tabular}{|c|c|c|c|c|c|c|}
\hline Index & Phylum & Family & Species & Host & Location & References \\
\hline 1465 & & & $\begin{array}{l}\text { Tetraphyllidean cercoids (bilocular } \\
\text { bothria) }\end{array}$ & Channichthys rhinoceratus & Heard Island Plateau & Wojciechowska et al. (1995) \\
\hline 1466 & & & $\begin{array}{c}\text { Tetraphyllidean cercoids (bilocular } \\
\text { bothria) }\end{array}$ & Notothenia squamifrons & Heard Island Plateau & Wojciechowska et al. (1995) \\
\hline 1467 & & & $\begin{array}{l}\text { Tetraphyllidean cercoids (monolocular } \\
\text { bothria) }\end{array}$ & Champsocephalus gunnari & Heard Island Plateau & Wojciechowska et al. (1995) \\
\hline 1468 & & & $\begin{array}{l}\text { Tetraphyllidean cercoids (monolocular } \\
\text { bothria) }\end{array}$ & Channichthys rhinoceratus & Heard Island Plateau & Wojciechowska et al. (1995) \\
\hline 1469 & & & $\begin{array}{l}\text { Tetraphyllidean cercoids unidentified } \\
\text { bothria and hook-like projections }\end{array}$ & Champsocephalus gunnari & Heard Island Plateau & Wojciechowska et al. (1995) \\
\hline 1470 & & & $\begin{array}{l}\text { Tetraphyllidean cercoids unidentified } \\
\text { bothria and hook-like projections }\end{array}$ & Channichthys rhinoceratus & Heard Island Plateau & Wojciechowska et al. (1995) \\
\hline 1471 & & & Tetraphyllidean plerocercoids & Dissostichus eleginoides & Shag Rocks & Brickle et al. (2005) \\
\hline 1472 & & & Tetraphyllidean plerocercoids & Dissostichus eleginoides & South Georgia Island & Brickle et al. (2005) \\
\hline 1473 & & & Tetraphyllidean plerocercoids & Dissostichus eleginoides & Prince Edward Island & Brickle et al. (2005) \\
\hline 1474 & & & Tetraphyllidean plerocercoids & Dissostichus eleginoides & Macquarie Island & Brickle et al. (2005) \\
\hline 1475 & & & Tetraphyllidean plerocercoids & Dissostichus eleginoides & Ross Sea & Brickle et al. (2005) \\
\hline 1476 & & & Tetraphyllidean plerocercoids & Dissostichus eleginoides & Falkland Islands & Brickle et al. (2005) \\
\hline 1477 & & & Tetraphyllidean sp. (larvae) & Notothenia coriiceps & $\begin{array}{l}\text { Potter Cove (King George } \\
\text { Island) }\end{array}$ & Palm et.al. (1998) \\
\hline 1478 & \multirow{12}{*}{ CESTODA } & Oncobothriidae & Tetraphyllidean sp. (larvae) & Notothenia coriiceps & King George Island & Palm et.al. (1998) \\
\hline 1479 & & & Trilocular cercoids & Gymnodraco acuticeps & South Shetland Islands & Laskowski et.al. (2005) \\
\hline 1480 & & & Triocular metacestode & Trematomus newnesi & $\begin{array}{l}\text { Adelie Land (Eastern } \\
\text { Antarctica) }\end{array}$ & Laskowski et.al. (2007) \\
\hline 1481 & & & $\begin{array}{c}\text { Tetraphyllidean Cercoid With Bilocular } \\
\text { Bothridia (Phyllobothriidae) }\end{array}$ & Chionodraco hamatus & Adelie Land & Zdzitowiecki et al. (1998) \\
\hline 1482 & & & $\begin{array}{c}\text { Tetraphyllidean Cercoid With Bilocular } \\
\text { Bothridia (Phyllobothriidae) }\end{array}$ & Notothenia coriiceps & Adelie Land & Zdzitowiecki et al. (1998) \\
\hline 1483 & & & $\begin{array}{c}\text { Tetraphyllidean Cercoid With Bilocular } \\
\text { Bothridia (Phyllobothriidae) }\end{array}$ & Trematomus bernacchii & Adelie Land & Zdzitowiecki et al. (1998) \\
\hline 1484 & & & $\begin{array}{c}\text { Tetraphyllidean Cercoid With Bilocular } \\
\text { Bothridia (Phyllobothriidae) }\end{array}$ & Trematomus hansoni & Adelie Land & Zdzitowiecki et al. (1998) \\
\hline 1485 & & & $\begin{array}{c}\text { Tetraphyllidean Cercoid With Trilocular } \\
\text { Bothridia (Onchobothriidae) }\end{array}$ & Chionodraco hamatus & Adelie Land & Zdzitowiecki et al. (1998) \\
\hline 1486 & & & $\begin{array}{c}\text { Tetraphyllidean Cercoid With Trilocular } \\
\text { Bothridia (Onchobothriidae) }\end{array}$ & Notothenia coriiceps & Adelie Land & Zdzitowiecki et al. (1998) \\
\hline 1487 & & & $\begin{array}{c}\text { Tetraphyllidean Cercoid With Trilocular } \\
\text { Bothridia (Onchobothriidae) }\end{array}$ & Trematomus hansoni & Adelie Land & Zdzitowiecki et al. (1998) \\
\hline 1488 & & Bothriocephalidae & Bothriocephalus sp. & blank & blank & Rudolphi (1808) \\
\hline 1489 & & & Bothriocephalus sp. & HOST NAME NOT MENTIONED & Kerguelen Subregion & Lyadov (1985) \\
\hline
\end{tabular}




\begin{tabular}{|c|c|c|c|c|c|c|}
\hline Index & Phylum & Family & Species & Host & Location & References \\
\hline 1490 & & & Bothriocephalus sp. & blank & blank & Wojciechowska et al. (1995) \\
\hline 1491 & & & Bothriocephalus antarcticus & Champsocephalus gunnari & Heard Island Plateau & Wojciechowska et al. (1995) \\
\hline 1492 & & & Bothriocephalus antarcticus & Channichthys rhinoceratus & Heard Island Plateau & Wojciechowska et al. (1995) \\
\hline 1493 & & & Bothriocephalus antarcticus & blank & blank & Rudolphi (1808) \\
\hline 1494 & & & Clestobothrium crassiceps & Dissostichus eleginoides & Falkland Islands & Brickle et al. (2006) \\
\hline 1495 & & & Clestobothrium crassiceps & Dissostichus eleginoides & South Georgia Island & Gaevskaya et al. (1990) \\
\hline 1496 & & & Clestobothrium crassiceps & Merluccius hubbsi & Patagonian Shelf & Reimer \& Jessen (1974) \\
\hline 1497 & & Caryophyllaeidae & Monobothrium sp. & Bathyraja eatoni & Heard Island Plateau & Wojciechowska et al. (1995) \\
\hline 1498 & & Diphyllobothriidae & Dipyllobothriid plerocercoid & Lepidonotothen larseni & $\begin{array}{l}\text { Port Foster (Deception } \\
\text { Island) }\end{array}$ & Ruhl et al. (2003) \\
\hline 1499 & & & Dipyllobothriid plerocercoid & Lepidonotothen nudifrons & $\begin{array}{l}\text { Port Foster (Deception } \\
\text { Island) }\end{array}$ & Ruhl et al. (2003) \\
\hline 1500 & & & Dipyllobothriid plerocercoid & Trematomus bernacchii & $\begin{array}{l}\text { Port Foster (Deception } \\
\text { Island) }\end{array}$ & Ruhl et al. (2003) \\
\hline 1501 & & & Dipyllobothriid plerocercoid & Trematomus scottii & $\begin{array}{l}\text { Port Foster (Deception } \\
\text { Island) }\end{array}$ & Ruhl et al. (2003) \\
\hline 1502 & & & Diphyllobothriid plerocercoids & Chionodraco hamatus & Adelie Land & Zdzitowiecki et.al. (1998) \\
\hline 1503 & \multirow{13}{*}{ CESTODA } & & Diphyllobothriid plerocercoids & blank & blank & Zdzitowiecki (2001b) \\
\hline 1504 & & Diphyllobothriidae & Diphyllobothriid plerocercoids & Dissostichus eleginoides & Heard Island Plateau & Wojciechowska et al. (1995) \\
\hline 1505 & & & Diphyllobothriid plerocercoids & Gymnodraco acuticeps & Ross Sea & Laskowski et.al. (2005) \\
\hline 1506 & & & Diphyllobothriid plerocercoids & Gymnodraco acuticeps & South Shetland Islands & Laskowski et.al. (2005) \\
\hline 1507 & & & Diphyllobothriid plerocercoids & Notothenia coriiceps & Adelie Land & Zdzitowiecki et.al. (1998) \\
\hline 1508 & & & Diphyllobothriid plerocercoids & Notothenia coriiceps & Adelie Land & Zdzitowiecki (2001b) \\
\hline 1509 & & & Diphyllobothriid plerocercoids & Notothenia coriiceps & Vernadsky & $\begin{array}{c}\text { Zdzitowiecki \& Laskowski } \\
\text { (2004) }\end{array}$ \\
\hline 1510 & & & Diphyllobothriid plerocercoids & Notothenia squamifrons & Heard Island Plateau & Wojciechowska et al. (1995) \\
\hline 1511 & & & Diphyllobothriid plerocercoids & Parachaenichthys charcoti & $\begin{array}{l}\text { Off the South Shetland } \\
\text { Islands }\end{array}$ & Zdzitowiecki (2001a) \\
\hline 1512 & & & Diphyllobothriid plerocercoids & Trematomus bernacchii & Adelie Land & Zdzitowiecki et.al. (1998) \\
\hline 1513 & & & Diphyllobothriid plerocercoids & Trematomus hansoni & Adelie Land & Zdzitowiecki et.al. (1998) \\
\hline 1514 & & & Diphyllobothriid plerocercoids & Trematomus newnesi & $\begin{array}{l}\text { Adelie Land (Eastern } \\
\text { Antarctica) }\end{array}$ & Laskowski et.al. (2007) \\
\hline 1515 & & & Diphyllobothrium $s p$. & blank & blank & Cobbold (1858) \\
\hline
\end{tabular}




\begin{tabular}{|c|c|c|c|c|c|c|}
\hline Index & Phylum & Family & Species & Host & Location & References \\
\hline 1516 & & & Diphyllobothrium sp. & Chaenocephalus aceratus & Georgia & Parukhin \& Lyadov (1981) \\
\hline 1517 & & & Diphyllobothrium sp. & Chaenichthys rhinoceratus & Kerguelen Subregion & Parukhin \& Lyadov (1981) \\
\hline 1518 & & & Diphyllobothrium sp. & blank & blank & Lyadov et al. (1981) \\
\hline 1519 & & & Diphyllobothrium sp. & Notothenia coriiceps & $\begin{array}{l}\text { Potter Cove (King George } \\
\text { Island) }\end{array}$ & Palm et.al. (1998) \\
\hline 1520 & & & Diphyllobothrium sp. & Notothenia coriiceps & King George Island & Palm et.al. (1998) \\
\hline 1521 & & & Diphyllobothrium sp. & Notothenia gibberifrons & Georgia & Parukhin \& Lyadov (1981) \\
\hline 1522 & & & Diphyllobothrium sp. & Notothenia neglecta & Archipelago Melcihor & Szidat (1965) \\
\hline 1523 & & & Diphyllobothrium sp. & Notothenia rossii & Georgia & Parukhin \& Lyadov (1981) \\
\hline 1524 & & & Diphyllobothrium sp. & Trematomus bernacchii & Mcmurdo Sound & Moser \& Cowen (1991) \\
\hline 1525 & & Echinobothriidae & Echinobothrium acanthocolle & blank & blank & Wojciechowska (1991) \\
\hline 1526 & & & Echinobothrium acanthocolle & Raja georgiana & South Georgia (Shelves) & Rocka (2003) \\
\hline 1527 & & Echinophallidae & Neobothriocephalus sp. & blank & blank & Mateo \& Bullock (1966) \\
\hline 1528 & & & Neobothriocephalus sp. & HOST NAME NOT MENTIONED & Kerguelen Subregion & Lyadov (1985) \\
\hline 1529 & & & Parabothriocephalus johnstoni & blank & blank & Prudhoe (1969) \\
\hline 1530 & & & Parabothriocephalus johnstoni & Macrourus whitsoni & Weddell Sea & Rocka \& Zdzitowiecki (1998) \\
\hline 1531 & \multirow{11}{*}{ CESTODA } & & Parabothriocephalus macruri & blank & blank & $\begin{array}{l}\text { Campbell, Correita \& Haedrish } \\
\text { (1982) }\end{array}$ \\
\hline 1532 & & Echinophallidae & Parabothriocephalus macruri & Macrourus carinatus & $\begin{array}{l}\text { Falkland (Malvinas) } \\
\text { Islands }\end{array}$ & Gaevskaya \& Rodyuk (1988) \\
\hline 1533 & & Lacistorhynchidae & Grillotia sp. & blank & blank & Von Nordmann (1832) \\
\hline 1534 & & & Grillotia sp. & Merluccius hubbsi & Patagonian Shelf & Reimer \& Jessen (1974) \\
\hline 1535 & & & Grillotia erinaceus & blank & blank & van Beneden (1858) \\
\hline 1536 & & & Grillotia erinaceus & Dissostichus eleginoides & South Georgia Island & Brickle et al. (2005) \\
\hline 1537 & & & Grillotia erinaceus & Dissostichus eleginoides & Macquarie Island & Brickle et al. (2005) \\
\hline 1538 & & & Grillotia erinaceus & Dissostichus eleginoides & Falkland Islands & Brickle et al. (2006) \\
\hline 1539 & & & Grillotia erinaceus larva & blank & blank & van Beneden (1858) \\
\hline 1540 & & & Grillotia erinaceus larva & Dissostichus eleginoides & Falkland-Patagonia & Gaevskaya et al. (1990) \\
\hline 1541 & & & Grillotia erinaceus larva & Macrourus carinatus & $\begin{array}{l}\text { Falkland (Malvinas) } \\
\text { Islands }\end{array}$ & Gaevskaya \& Rodyuk (1988) \\
\hline
\end{tabular}




\begin{tabular}{|c|c|c|c|c|c|c|}
\hline Index & Phylum & Family & Species & Host & Location & References \\
\hline 1542 & & & Lacistorhynchus tenuis & blank & blank & van Beneden (1858) \\
\hline 1543 & & & Lacistorhynchus tenuis & Macrourus carinatus & $\begin{array}{l}\text { Falkland (Malvinas) } \\
\text { Islands }\end{array}$ & Gaevskaya \& Rodyuk (1988) \\
\hline 1544 & & & Lacistorhynchus tenuis & Dissostichus eleginoides & Falkland-Patagonia & Gaevskaya et al. (1990) \\
\hline 1545 & & Onchobothriidae & Acantobothrium sp. & blank & blank & van Beneden (1850) \\
\hline 1546 & & & Acantobothrium sp. & HOST NAME NOT MENTIONED & Kerguelen Subregion & Lyadov (1985) \\
\hline 1547 & & & Oncobothrium antarcticum & blank & blank & Wojciechowska (1990) \\
\hline 1548 & & & Oncobothrium antarcticum & Bathyraja maccaini & Weddell Sea & Rocka \& Zdzitowiecki (1998) \\
\hline 1549 & & Phyllobothriidae & $\begin{array}{l}\text { Phyllobothriidae (Tetraphyllidean } \\
\text { cercoid with bilocular bothridia) }\end{array}$ & Chionodraco hamatus & Adelie Land & Zdzitowiecki et.al. (1998) \\
\hline 1550 & & & $\begin{array}{l}\text { Phyllobothriidae (Tetraphyllidean } \\
\text { cercoid with bilocular bothridia) }\end{array}$ & Notothenia coriiceps & Adelie Land & Zdzitowiecki et.al. (1998) \\
\hline 1551 & & & $\begin{array}{l}\text { Phyllobothriidae (Tetraphyllidean } \\
\text { cercoid with bilocular bothridia) }\end{array}$ & Trematomus bernacchii & Adelie Land & Zdzitowiecki et.al. (1998) \\
\hline 1552 & & & $\begin{array}{l}\text { Phyllobothriidae (Tetraphyllidean } \\
\text { cercoid with bilocular bothridia) }\end{array}$ & Trematomus hansoni & Adelie Land & Zdzitowiecki et.al. (1998) \\
\hline 1553 & & & Marsupiobothrium awii & blank & blank & Rocka \& Zdzitowiecki (1998) \\
\hline 1554 & & & Marsupiobothrium awii & Bathyraja maccaini & Weddell Sea & Rocka \& Zdzitowiecki (1998) \\
\hline 1555 & & & Phyllobothrium sp. & blank & blank & van Beneden (1849) \\
\hline 1556 & & & Phyllobothrium sp. & Bathyraja eatoni & Heard Island Plateau & Wojciechowska et al. (1995) \\
\hline 1557 & & & Phyllobothrium sp. & Chaenichthys velifer & Kerguelen Subregion & Lyadov et al. (1981) \\
\hline 1558 & & & Phyllobothrium sp. & blank & blank & Parukhin \& Lyadov (1981) \\
\hline 1559 & \multirow{9}{*}{ CESTODA } & & Phyllobothrium sp. & Chaenocephalus aceratus & Georgia & Parukhin \& Lyadov (1981) \\
\hline 1560 & & Phyllobothriidae & Phyllobothrium sp. & Champsocephalus gunnari & Kerguelen Subregion & Parukhin \& Lyadov (1981) \\
\hline 1561 & & & Phyllobothrium sp. & Champsocephalus gunnari & Skif Bank & Parukhin \& Lyadov (1981) \\
\hline 1562 & & & Phyllobothrium sp. & Champsocephalus gunnari & Georgia & Parukhin \& Lyadov (1981) \\
\hline 1563 & & & Phyllobothrium sp. & Champsocephalus gunnari & Kerguelen Subregion & Lyadov et al. (1981) \\
\hline 1564 & & & Phyllobothrium sp. & Champsocephalus gunnari & Skif Bank & Lyadov et al. (1981) \\
\hline 1565 & & & Phyllobothrium sp. & Dissostichus eleginoides & Kerguelen Subregion & Parukhin \& Lyadov (1981) \\
\hline 1566 & & & Phyllobothrium sp. & Dissostichus eleginoides & Skif Bank & Parukhin \& Lyadov (1981) \\
\hline 1567 & & & Phyllobothrium sp. & Dissostichus eleginoides & Kerguelen Subregion & Parukhin \& Lyadov (1982) \\
\hline
\end{tabular}




\begin{tabular}{|c|c|c|c|c|c|c|}
\hline Index & Phylum & Family & Species & Host & Location & References \\
\hline 1568 & & & Phyllobothrium sp. & Dissostichus eleginoides & Skif Bank & Parukhin \& Lyadov (1982) \\
\hline 1569 & & & Phyllobothrium sp. & Dissostichus eleginoides & Ob Bank & Parukhin \& Lyadov (1982) \\
\hline 1570 & & & Phyllobothrium sp. & Notothenia acuta & Kerguelen Subregion & Parukhin \& Lyadov (1981) \\
\hline 1571 & & & Phyllobothrium sp. & Notothenia acuta & Kerguelen Subregion & Parukhin \& Lyadov (1982) \\
\hline 1572 & & & Phyllobothrium sp. & Notothenia kempi & Shag Rocks & Parukhin \& Lyadov (1981) \\
\hline 1573 & & & Phyllobothrium sp. & Notothenia mizops & Kerguelen Subregion & Parukhin \& Lyadov (1981) \\
\hline 1574 & & & Phyllobothrium sp. & blank & blank & Parukhin \& Lyadov (1982) \\
\hline 1575 & & & Phyllobothrium sp. & Notothenia rossii & Georgia & Parukhin \& Lyadov (1981) \\
\hline 1576 & & & Phyllobothrium sp. & Nototheniidae & Kerguelen Subregion & Parukhin \& Lyadov (1982) \\
\hline 1577 & & & Phyllobothrium sp. & Pseudochaenichthys georgianus & Georgia & Parukhin \& Lyadov (1981) \\
\hline 1578 & & & Phyllobothrium sp. & Trematomus bernacchii & Mcmurdo Sound & Moser \& Cowen (1991) \\
\hline 1579 & & & Phyllobothrium sp. & HOST NAME NOT MENTIONED & Kerguelen Subregion & Lyadov (1985) \\
\hline 1580 & & & Phyllobothrium sp. & HOST NAME NOT MENTIONED & South Australia & Lyadov (1985) \\
\hline 1581 & & & Phyllobothrium sp. Larva & blank & blank & van Beneden (1849) \\
\hline 1582 & & & Phyllobothrium sp. Larva & Dissostichus eleginoides & South Georgia Island & Gaevskaya et al. (1990) \\
\hline 1583 & & & Phyllobothrium sp. Larva & Macrourus carinatus & $\begin{array}{l}\text { Falkland (Malvinas) } \\
\text { Islands }\end{array}$ & Gaevskaya \& Rodyuk (1988) \\
\hline 1584 & & & Phyllobothrium cercoid I & Champsocephalus gunnari & South Shetland Islands & Wojciechowska (1993b) \\
\hline 1585 & & & Phyllobothrium cercoid I & Chiondraco rastrospinosus & South Shetland Islands & Wojciechowska (1993b) \\
\hline 1586 & & & Phyllobothrium cercoid I & Electrona antarctica & South Shetland Islands & Wojciechowska (1993b) \\
\hline 1587 & & & Phyllobothrium cercoid I & Notothenia neglecta & South Shetland Islands & Wojciechowska (1993b) \\
\hline 1588 & & & Phyllobothrium cercoid I & Notothenia rossii & South Shetland Islands & Wojciechowska (1993b) \\
\hline 1589 & \multirow{6}{*}{ CESTODA } & Phyllobothriidae & Phyllobothrium cercoid I & Nototheniops nudifrons & South Shetland Islands & Wojciechowska (1993b) \\
\hline 1590 & & & Phyllobothrium cercoid I & Paradiplospinus antarcticus & South Shetland Islands & Wojciechowska (1993b) \\
\hline 1591 & & & Phyllobothrium cercoid I & Protomyctophum bolini & South Shetland Islands & Wojciechowska (1993b) \\
\hline 1592 & & & Phyllobothrium cercoid I & Pseudochaenichthys georgianus & South Shetland Islands & Wojciechowska (1993b) \\
\hline 1593 & & & Phyllobothrium cercoid I & Trematomus newnesi & South Shetland Islands & Wojciechowska (1993b) \\
\hline 1594 & & & Phyllobothrium cercoid II & Chaenocephalus aceratus & South Georgia Island & Wojciechowska (1993b) \\
\hline
\end{tabular}




\begin{tabular}{|c|c|c|c|c|c|c|}
\hline Index & Phylum & Family & Species & Host & Location & References \\
\hline 1595 & & & Phyllobothrium cercoid II & Champsocephalus gunnari & South Georgia Island & Wojciechowska (1993b) \\
\hline 1596 & & & Phyllobothrium cercoid II & Dissostichus eleginoides & South Georgia Island & Wojciechowska (1993b) \\
\hline 1597 & & & Phyllobothrium cercoid II & Notothenia rossii & South Georgia Island & Wojciechowska (1993b) \\
\hline 1598 & & & Phyllobothrium cercoid II & Notothenia squamifrons & South Georgia Island & Wojciechowska (1993b) \\
\hline 1599 & & & Phyllobothrium cercoid II & Patagonotothen brevicaudata & South Georgia Island & Wojciechowska (1993b) \\
\hline 1600 & & & Phyllobothrium cercoid II & Pseudochaenichthys georgianus & South Georgia Island & Wojciechowska (1993b) \\
\hline 1601 & & & Phyllobothrium cercoid III & Electrona carlsbergi & South Georgia Island & Wojciechowska (1993b) \\
\hline 1602 & & & Phyllobothrium cercoid III & Gymnocephalus sp. & North \& South & Wojciechowska (1993b) \\
\hline 1603 & & & Phyllobothrium cercoid III & Gymnocephalus sp. & South Shetland Islands & Wojciechowska (1993b) \\
\hline 1604 & & & Phyllobothrium cercoid III & Gymnocephalus nicholsi & North \& South & Wojciechowska (1993b) \\
\hline 1605 & & & Phyllobothrium cercoid III & Gymnocephalus nicholsi & South Shetland Islands & Wojciechowska (1993b) \\
\hline 1606 & & & Phyllobothrium cercoid IV & Chaenodraco wilsoni & South Shetland Islands & Wojciechowska (1993b) \\
\hline 1607 & & & Phyllobothrium cercoid IV & Chaenocephalus aceratus & South Shetland Islands & Wojciechowska (1993b) \\
\hline 1608 & & & Phyllobothrium cercoid IV & Champsocephalus gunnari & South Shetland Islands & Wojciechowska (1993b) \\
\hline 1609 & & & Phyllobothrium cercoid IV & Chiondraco rastrospinosus & South Shetland Islands & Wojciechowska (1993b) \\
\hline 1610 & & & Phyllobothrium cercoid IV & Cryodraco antarcticus & South Shetland Islands & Wojciechowska (1993b) \\
\hline 1611 & & & Phyllobothrium cercoid IV & Dissostichus eleginoides & South Shetland Islands & Wojciechowska (1993b) \\
\hline 1612 & & & Phyllobothrium cercoid IV & Dissostichus mawsoni & South Shetland Islands & Wojciechowska (1993b) \\
\hline 1613 & & & Phyllobothrium cercoid IV & Electrona antarctica & South Shetland Islands & Wojciechowska (1993b) \\
\hline 1614 & & & Phyllobothrium cercoid IV & Gymnodraco acuticeps & South Shetland Islands & Wojciechowska (1993b) \\
\hline 1615 & & & Phyllobothrium cercoid IV & Neopagetopsis ionah & South Shetland Islands & Wojciechowska (1993b) \\
\hline 1616 & & & Phyllobothrium cercoid IV & Notothenia gibberifrons & South Shetland Islands & Wojciechowska (1993b) \\
\hline 1617 & & & Phyllobothrium cercoid IV & Notothenia neglecta & South Shetland Islands & Wojciechowska (1993b) \\
\hline 1618 & \multirow{4}{*}{ CESTODA } & Phyllobothriidae & Phyllobothrium cercoid IV & Notothenia rossii & South Shetland Islands & Wojciechowska (1993b) \\
\hline 1619 & & & Phyllobothrium cercoid IV & Notothenia squamifrons & South Shetland Islands & Wojciechowska (1993b) \\
\hline 1620 & & & Phyllobothrium cercoid IV & Nototheniops nudifrons & South Shetland Islands & Wojciechowska (1993b) \\
\hline 1621 & & & Phyllobothrium cercoid IV & Nototheniops nybelini & South Shetland Islands & Wojciechowska (1993b) \\
\hline
\end{tabular}




\begin{tabular}{|c|c|c|c|c|c|c|}
\hline Index & Phylum & Family & Species & Host & Location & References \\
\hline 1622 & & & Phyllobothrium cercoid IV & Pagothenia bernacchi & South Shetland Islands & Wojciechowska (1993b) \\
\hline 1623 & & & Phyllobothrium cercoid IV & Pagothenia brachysoma & South Shetland Islands & Wojciechowska (1993b) \\
\hline 1624 & & & Phyllobothrium cercoid IV & Pagothenia hansoni & South Shetland Islands & Wojciechowska (1993b) \\
\hline 1625 & & & Phyllobothrium cercoid IV & Parachaenichthys charcoti & South Shetland Islands & Wojciechowska (1993b) \\
\hline 1626 & & & Phyllobothrium cercoid IV & Pleurogramma antarcticum & South Shetland Islands & Wojciechowska (1993b) \\
\hline 1627 & & & Phyllobothrium cercoid IV & Pseudochaenichthys georgianus & South Shetland Islands & Wojciechowska (1993b) \\
\hline 1628 & & & Phyllobothrium cercoid IV & Psilodraco sp. & South Shetland Islands & Wojciechowska (1993b) \\
\hline 1629 & & & Phyllobothrium cercoid IV & Racovitzia glacialis & South Shetland Islands & Wojciechowska (1993b) \\
\hline 1630 & & & Phyllobothrium cercoid IV & Trematomus eulepidotus & South Shetland Islands & Wojciechowska (1993b) \\
\hline 1631 & & & Phyllobothrium cercoid IV & Trematomus newnesi & South Shetland Islands & Wojciechowska (1993b) \\
\hline 1632 & & & Phyllobothrium cercoid $V$ & Artedidraco mirus & South Georgia Island & Wojciechowska (1993b) \\
\hline 1633 & & & Phyllobothrium cercoid $V$ & Chaenocephalus aceratus & South Georgia Island & Wojciechowska (1993b) \\
\hline 1634 & & & Phyllobothrium cercoid V & Champsocephalus gunnari & South Georgia Island & Wojciechowska (1993b) \\
\hline 1635 & & & Phyllobothrium cercoid $V$ & Dissostichus eleginoides & South Georgia Island & Wojciechowska (1993b) \\
\hline 1636 & & & Phyllobothrium cercoid V & Muraenolepis microps & South Georgia Island & Wojciechowska (1993b) \\
\hline 1637 & & & Phyllobothrium cercoid $V$ & Notothenia gibberifrons & South Georgia Island & Wojciechowska (1993b) \\
\hline 1638 & & & Phyllobothrium cercoid V & Notothenia rossii & South Georgia Island & Wojciechowska (1993b) \\
\hline 1639 & & & Phyllobothrium cercoid V & Notothenia squamifrons & South Georgia Island & Wojciechowska (1993b) \\
\hline 1640 & & & Phyllobothrium cercoid $V$ & Nototheniops larseni & South Georgia Island & Wojciechowska (1993b) \\
\hline 1641 & & & Phyllobothrium cercoid $V$ & Nototheniops nudifrons & South Georgia Island & Wojciechowska (1993b) \\
\hline 1642 & & & Phyllobothrium cercoid $V$ & Pagothenia hansoni & South Georgia Island & Wojciechowska (1993b) \\
\hline 1643 & & & Phyllobothrium cercoid $V$ & Parachaenichthys georgianus & South Georgia Island & Wojciechowska (1993b) \\
\hline 1644 & & & Phyllobothrium cercoid $V$ & Patagonotothen brevicauda & South Georgia Island & Wojciechowska (1993b) \\
\hline 1645 & & & Phyllobothrium cercoid V & Pseudochaenichthys georgianus & South Georgia Island & Wojciechowska (1993b) \\
\hline 1646 & & & Phyllobothrium cercoid $V$ & Psilodraco breviceps & South Georgia Island & Wojciechowska (1993b) \\
\hline 1647 & СБСТОРА & Phyllobothriidae & Phyllobothrium cercoid VI & Chaenocephalus aceratus & South Shetland Islands & Wojciechowska (1993b) \\
\hline 1648 & CESIUDA & & Phyllobothrium cercoid VI & Chaenodraco wilsoni & South Shetland Islands & Wojciechowska (1993b) \\
\hline
\end{tabular}




\begin{tabular}{|c|c|c|c|c|c|c|}
\hline Index & Phylum & Family & Species & Host & Location & References \\
\hline 1649 & & & Phyllobothrium cercoid VI & Champsocephalus gunnari & South Shetland Islands & Wojciechowska (1993b) \\
\hline 1650 & & & Phyllobothrium cercoid VI & Chiondraco rastrospinosus & South Shetland Islands & Wojciechowska (1993b) \\
\hline 1651 & & & Phyllobothrium cercoid VI & Cryodraco antarcticus & South Shetland Islands & Wojciechowska (1993b) \\
\hline 1652 & & & Phyllobothrium cercoid VI & Dissostichus eleginoides & South Shetland Islands & Wojciechowska (1993b) \\
\hline 1653 & & & Phyllobothrium cercoid VI & Dissostichus mawsoni & South Shetland Islands & Wojciechowska (1993b) \\
\hline 1654 & & & Phyllobothrium cercoid VI & Gymnodraco acuticeps & South Shetland Islands & Wojciechowska (1993b) \\
\hline 1655 & & & Phyllobothrium cercoid VI & Gymnoscopelus nicholsi & South Shetland Islands & Wojciechowska (1993b) \\
\hline 1656 & & & Phyllobothrium cercoid VI & Neopagetopsis ionah & South Shetland Islands & Wojciechowska (1993b) \\
\hline 1657 & & & Phyllobothrium cercoid VI & Notothenia gibberifrons & South Shetland Islands & Wojciechowska (1993b) \\
\hline 1658 & & & Phyllobothrium cercoid VI & Notothenia neglecta & South Shetland Islands & Wojciechowska (1993b) \\
\hline 1659 & & & Phyllobothrium cercoid VI & Notothenia rossii & South Shetland Islands & Wojciechowska (1993b) \\
\hline 1660 & & & Phyllobothrium cercoid VI & Nototheniops nudifrons & South Shetland Islands & Wojciechowska (1993b) \\
\hline 1661 & & & Phyllobothrium cercoid VI & Nototheniops nybelini & South Shetland Islands & Wojciechowska (1993b) \\
\hline 1662 & & & Phyllobothrium cercoid VI & Pagothenia hansoni & South Shetland Islands & Wojciechowska (1993b) \\
\hline 1663 & & & Phyllobothrium cercoid VI & Pagothenia bernacchi & South Shetland Islands & Wojciechowska (1993b) \\
\hline 1664 & & & Phyllobothrium cercoid VI & Pagothenia brachysoma & South Shetland Islands & Wojciechowska (1993b) \\
\hline 1665 & & & Phyllobothrium cercoid VI & Parachaenichthys charcoti & South Shetland Islands & Wojciechowska (1993b) \\
\hline 1666 & & & Phyllobothrium cercoid VI & Pleurogramma antarcticum & South Shetland Islands & Wojciechowska (1993b) \\
\hline 1667 & & & Phyllobothrium cercoid VI & Pseudochaenichthys georgianus & South Shetland Islands & Wojciechowska (1993b) \\
\hline 1668 & & & Phyllobothrium cercoid VI & Psilodraco sp. & South Shetland Islands & Wojciechowska (1993b) \\
\hline 1669 & & & Phyllobothrium cercoid VI & Trematomus newnesi & South Shetland Islands & Wojciechowska (1993b) \\
\hline 1670 & & & Phyllobothrium cercoid VI & Trematomus eulepidotus & South Shetland Islands & Wojciechowska (1993b) \\
\hline 1671 & & & Phyllobothrium cercoid VII & Champsocephalus gunnari & Shag Rocks & Wojciechowska (1993b) \\
\hline 1672 & & & Phyllobothrium cercoid VII & Dissostichus eleginoides & South Georgia Island & Wojciechowska (1993b) \\
\hline 1673 & & & Phyllobothrium cercoid VII & Patagonotothen brevicaudata guntheri & South Georgia Island & Wojciechowska (1993b) \\
\hline 1674 & & & Phyllobothrium cercoid VIII & Champsocephalus gunnari & $\begin{array}{c}\text { Cumberland Bay (South } \\
\text { Georgia) }\end{array}$ & Wojciechowska (1993b) \\
\hline 1675 & & & Phyllobothrium cercoid VIII & Harpagifer georgianus & $\begin{array}{c}\text { Cumberland Bay (South } \\
\text { Georgia) }\end{array}$ & Wojciechowska (1993b) \\
\hline
\end{tabular}




\begin{tabular}{|c|c|c|c|c|c|c|}
\hline Index & Phylum & Family & Species & Host & Location & References \\
\hline 1676 & & Phyllobothriidae & Phyllobothrium cercoid VIII & Pagothenia hansoni & $\begin{array}{c}\text { Cumberland Bay (South } \\
\text { Georgia) }\end{array}$ & Wojciechowska (1993b) \\
\hline 1677 & & & Pseudanthobothrium notogeorgianum & blank & blank & Wojciechowska (1990) \\
\hline 1678 & & & Pseudanthobothrium notogeorgianum & Raja georgiana & South Georgia Island & Wojciechowska (1990) \\
\hline 1679 & & & Pseudanthobothrium shetlandicum & blank & blank & Wojciechowska (1990) \\
\hline 1680 & & & Pseudanthobothrium shetlandicum & Bathyraja eatoni & South Shetland Islands & Wojciechowska (1990) \\
\hline 1681 & & & Pseudanthobothrium shetlandicum & Bathyraja eatoni & Joinville Shelf & Wojciechowska (1990) \\
\hline 1682 & & & Pseudanthobothrium shetlandicum & Bathyraja eatoni & Elephant Island Shelf & Wojciechowska (1990) \\
\hline 1683 & & & Pseudanthobothrium shetlandicum & Bathyraja eatoni & Admiralty Bay & Wojciechowska (1990) \\
\hline 1684 & & & Pseudanthobothrium shetlandicum & Bathyraja maccaini & South Shetland Islands & Wojciechowska (1990) \\
\hline 1685 & & & Pseudanthobothrium shetlandicum & Bathyraja maccaini & Joinville Shelf & Wojciechowska (1990) \\
\hline 1686 & & & Pseudanthobothrium shetlandicum & Bathyraja maccaini & Elephant Island Shelf & Wojciechowska (1990) \\
\hline 1687 & & & Pseudanthobothrium shetlandicum & Bathyraja maccaini & Admiralty Bay & Wojciechowska (1990) \\
\hline 1688 & & Rhinobothriidae & Anthocephalum arctowskii & blank & blank & Wojciechowska (1991) \\
\hline 1689 & CESTODA & & Anthocephalum arctowskii & Bathyraja sp. & Weddell Sea & Rocka \& Zdzitowiecki (1998) \\
\hline 1690 & & & Anthocephalum siedleckii & blank & blank & Wojciechowska (1991) \\
\hline 1691 & & & Anthocephalum siedleckii & Bathyraja maccaini & Weddell Sea & Rocka \& Zdzitowiecki (1998) \\
\hline 1692 & & Sphyriocephalidae & Hepatoxylon sp. Larvae & blank & blank & Bosc (1811) \\
\hline 1693 & & & Hepatoxylon sp. Larvae & HOST NAME NOT MENTIONED & Kerguelen Subregion & Lyadov (1985) \\
\hline 1694 & & & Hepatoxylon sp. Larvae & HOST NAME NOT MENTIONED & South Australia & Lyadov (1985) \\
\hline 1695 & & & Hepatoxylon trichiuri & HOST NAME NOT MENTIONED & South Australia & Holten (1802) \\
\hline 1696 & & & Hepatoxylon trichiuri & Dissostichus eleginoides & Prince Edward Island & Brickle et al. (2005) \\
\hline 1697 & & & Hepatoxylon trichiuri & Dissostichus eleginoides & Heard Island & Brickle et al. (2005) \\
\hline 1698 & & & Hepatoxylon trichiuri & Dissostichus eleginoides & Macquarie Island & Brickle et al. (2005) \\
\hline 1699 & & & Hepatoxylon trichiuri & Dissostichus eleginoides & Falkland Islands & Brickle et al. (2006) \\
\hline 1700 & & $\begin{array}{c}\text { Tetraphyllidea incertea } \\
\text { sedis }\end{array}$ & Scolex sp. & blank & blank & Müller (1787) \\
\hline 1701 & & & Scolex sp. & Parachaenichthys charcoti & South Shetland Islands & Szidat \& Graefe (1967) \\
\hline 1702 & & & Scolex pleuronectis & blank & blank & Müller (1788) \\
\hline
\end{tabular}




\begin{tabular}{|c|c|c|c|c|c|c|}
\hline Index & Phylum & Family & Species & Host & Location & References \\
\hline 1703 & & & Scolex pleuronectis & Chaenichthys rhinoceratus & Kerguelen Subregion & Parukhin \& Lyadov (1981) \\
\hline 1704 & & & Scolex pleuronectis & blank & blank & Lyadov et al. (1981) \\
\hline 1705 & & $\begin{array}{l}\text { Tetraphyllidea incertea } \\
\text { sedis }\end{array}$ & Scolex pleuronectis & Chaenichthys velifer & Kerguelen Subregion & Parukhin \& Lyadov (1981) \\
\hline 1706 & & & Scolex pleuronectis & blank & blank & Lyadov et al. (1981) \\
\hline 1707 & & & Scolex pleuronectis & Champsocephalus gunnari & Kerguelen Subregion & Parukhin \& Lyadov (1981) \\
\hline 1708 & & & Scolex pleuronectis & Champsocephalus gunnari & Skif Bank & Parukhin \& Lyadov (1981) \\
\hline 1709 & & & Scolex pleuronectis & Champsocephalus gunnari & Georgia & Parukhin \& Lyadov (1981) \\
\hline 1710 & & & Scolex pleuronectis & Champsocephalus gunnari & Kerguelen Subregion & Lyadov et al. (1981) \\
\hline 1711 & & & Scolex pleuronectis & Champsocephalus gunnari & Skif Bank & Lyadov et al. (1981) \\
\hline 1712 & & & Scolex pleuronectis & Dissostichus eleginoides & Kerguelen Subregion & Parukhin \& Lyadov (1982) \\
\hline 1713 & & & Scolex pleuronectis & Dissostichus eleginoides & Skif Bank & Parukhin \& Lyadov (1982) \\
\hline 1714 & & & Scolex pleuronectis & Dissostichus eleginoides & Ob Bank & Parukhin \& Lyadov (1982) \\
\hline 1715 & & & Scolex pleuronectis & Dissostichus eleginoides & Lena Bank & Parukhin \& Lyadov (1982) \\
\hline 1716 & & & Scolex pleuronectis & Dissostichus eleginoides & Ob Bank & Parukhin (1986) \\
\hline 1717 & CESTODA & & Scolex pleuronectis & Dissostichus eleginoides & Lena Bank & Parukhin (1986) \\
\hline 1718 & & & Scolex pleuronectis & Dissostichus eleginoides & Kerguelen Subregion & Parukhin \& Lyadov (1981) \\
\hline 1719 & & & Scolex pleuronectis & Dissostichus eleginoides & Crozet Islands & Parukhin \& Lyadov (1981) \\
\hline 1720 & & & Scolex pleuronectis & Dissostichus eleginoides & Ob Bank & Parukhin \& Lyadov (1981) \\
\hline 1721 & & & Scolex pleuronectis & Dissostichus eleginoides & Lena Bank & Parukhin \& Lyadov (1981) \\
\hline 1722 & & & Scolex pleuronectis & Dissostichus eleginoides & Falkland Patagonia & Gaevskaya et al. (1990) \\
\hline 1723 & & & Scolex pleuronectis & Dissostichus eleginoides & South Georgia Island & Gaevskaya et al. (1990) \\
\hline 1724 & & & Scolex pleuronectis & Macrourus carinatus & $\begin{array}{l}\text { Falkland (Malvinas) } \\
\text { Islands }\end{array}$ & Gaevskaya \& Rodyuk (1988) \\
\hline 1725 & & & Scolex pleuronectis & Notothenia acuta & Kerguelen Subregion & Parukhin \& Lyadov (1981) \\
\hline 1726 & & & Scolex pleuronectis & blank & blank & Parukhin \& Lyadov (1982) \\
\hline 1727 & & & Scolex pleuronectis & Notothenia gibberifrons & Georgia & Parukhin \& Lyadov (1981) \\
\hline 1728 & & & Scolex pleuronectis & Notothenia larseni & Lena Bank & Parukhin (1986) \\
\hline 1729 & & & Scolex pleuronectis & Notothenia larseni & Ob Bank & Parukhin (1986) \\
\hline
\end{tabular}




\begin{tabular}{|c|c|c|c|c|c|c|}
\hline Index & Phylum & Family & Species & Host & Location & References \\
\hline 1730 & & & Scolex pleuronectis & Notothenia magellanica & Ob Bank & Parukhin (1986) \\
\hline 1731 & & & Scolex pleuronectis & Notothenia mizops & Kerguelen Subregion & Parukhin \& Lyadov (1981) \\
\hline 1732 & & & Scolex pleuronectis & blank & blank & Parukhin \& Lyadov (1982) \\
\hline 1733 & & & Scolex pleuronectis & Notothenia ramsayi & Folkland & Parukhin \& Lyadov (1981) \\
\hline 1734 & & $\begin{array}{l}\text { Tetraphyllidea incertea } \\
\text { sedis }\end{array}$ & Scolex pleuronectis & Notothenia rossii & Kerguelen Subregion & Parukhin \& Lyadov (1981) \\
\hline 1735 & & & Scolex pleuronectis & Notothenia rossii & Kherd Island & Parukhin \& Lyadov (1981) \\
\hline 1736 & & & Scolex pleuronectis & Notothenia rossii & Crozet Islands & Parukhin \& Lyadov (1981) \\
\hline 1737 & & & Scolex pleuronectis & Notothenia rossii & Skif Bank & Parukhin \& Lyadov (1981) \\
\hline 1738 & & & Scolex pleuronectis & Notothenia rossii & Ob Bank & Parukhin \& Lyadov (1981) \\
\hline 1739 & & & Scolex pleuronectis & Notothenia rossii & Georgia & Parukhin \& Lyadov (1981) \\
\hline 1740 & & & Scolex pleuronectis & Notothenia rossii & Kerguelen Subregion & Parukhin \& Lyadov (1982) \\
\hline 1741 & & & Scolex pleuronectis & Notothenia rossii & Crozet Islands & Parukhin \& Lyadov (1982) \\
\hline 1742 & & & Scolex pleuronectis & Notothenia rossii & Ob Bank & Parukhin \& Lyadov (1982) \\
\hline 1743 & & & Scolex pleuronectis & Notothenia rossii & Ob Bank & Parukhin (1986) \\
\hline 1744 & & & Scolex pleuronectis & Notothenia rossii & Lena Bank & Parukhin (1986) \\
\hline 1745 & CESTODA & & Scolex pleuronectis & Notothenia squamifrons & Kerguelen Subregion & Parukhin \& Lyadov (1981) \\
\hline 1746 & & & Scolex pleuronectis & Notothenia squamifrons & Crozet Islands & Parukhin \& Lyadov (1981) \\
\hline 1747 & & & Scolex pleuronectis & Notothenia squamifrons & Skif Bank & Parukhin \& Lyadov (1981) \\
\hline 1748 & & & Scolex pleuronectis & Notothenia squamifrons & Ob Bank & Parukhin \& Lyadov (1981) \\
\hline 1749 & & & Scolex pleuronectis & Notothenia squamifrons & Lena Bank & Parukhin \& Lyadov (1981) \\
\hline 1750 & & & Scolex pleuronectis & Notothenia squamifrons & Kerguelen Subregion & Parukhin \& Lyadov (1982) \\
\hline 1751 & & & Scolex pleuronectis & Notothenia squamifrons & Crozet Islands & Parukhin \& Lyadov (1982) \\
\hline 1752 & & & Scolex pleuronectis & Notothenia squamifrons & Skif Bank & Parukhin \& Lyadov (1982) \\
\hline 1753 & & & Scolex pleuronectis & Notothenia squamifrons & Ob Bank & Parukhin \& Lyadov (1982) \\
\hline 1754 & & & Scolex pleuronectis & Notothenia squamifrons & Lena Bank & Parukhin \& Lyadov (1982) \\
\hline 1755 & & & Scolex pleuronectis & Notothenia squamifrons & Lena Bank & Parukhin (1986) \\
\hline 1756 & & & Scolex pleuronectis & Nototheniidae & Kerguelen Subregion & Parukhin \& Lyadov (1982) \\
\hline
\end{tabular}




\begin{tabular}{|c|c|c|c|c|c|c|}
\hline Index & Phylum & Family & Species & Host & Location & References \\
\hline 1757 & & & Scolex pleuronectis & Parachaenichthys charcoti & South Shetland Islands & Szidat \& Graefe (1967) \\
\hline 1758 & & & Scolex pleuronectis & Pseudochaenichthys georgianus & Georgia & Parukhin \& Lyadov (1981) \\
\hline 1759 & & & Scolex pleuronectis & HOST NAME NOT MENTIONED & Glacial Subregion & Lyadov (1985) \\
\hline 1760 & & & Scolex pleuronectis & HOST NAME NOT MENTIONED & Kerguelen Subregion & Lyadov (1985) \\
\hline 1761 & & & Scolex pleuronectis & HOST NAME NOT MENTIONED & Patagonian Shelf & Lyadov (1985) \\
\hline 1762 & & & Scolex pleuronectis & HOST NAME NOT MENTIONED & South Australia & Lyadov (1985) \\
\hline 1763 & \multirow{19}{*}{ CESTODA } & Triaenophoridae & Eubothrium sp. & blank & blank & Nybelin (1922) \\
\hline 1764 & & & Eubothrium sp. & Notothenia rossii & Kerguelen Subregion & Parukhin \& Lyadov (1982) \\
\hline 1765 & & & Eubothrium sp. & Notothenia rossii & Crozet Islands & Parukhin \& Lyadov (1982) \\
\hline 1766 & & & Eubothrium sp. & Notothenia rossii & Ob Bank & Parukhin \& Lyadov (1982) \\
\hline 1767 & & & Eubothrium sp. & Notothenia rossii & Kerguelen Subregion & Parukhin \& Lyadov (1981) \\
\hline 1768 & & & Eubothrium sp. & Notothenia rossii & Kherd Island & Parukhin \& Lyadov (1981) \\
\hline 1769 & & & Eubothrium sp. & Notothenia rossii & Crozet Islands & Parukhin \& Lyadov (1981) \\
\hline 1770 & & & Eubothrium sp. & Notothenia rossii & Ob Bank & Parukhin \& Lyadov (1981) \\
\hline 1771 & & & Eubothrium sp. & Notothenia squamifrons & Kerguelen Subregion & Parukhin \& Lyadov (1981) \\
\hline 1772 & & & Eubothrium sp. & Notothenia squamifrons & Crozet Islands & Parukhin \& Lyadov (1981) \\
\hline 1773 & & & Eubothrium sp. & Notothenia squamifrons & Skif Bank & Parukhin \& Lyadov (1981) \\
\hline 1774 & & & Eubothrium sp. & Notothenia squamifrons & Ob Bank & Parukhin \& Lyadov (1981) \\
\hline 1775 & & & Eubothrium sp. & Notothenia squamifrons & Lena Bank & Parukhin \& Lyadov (1981) \\
\hline 1776 & & & Eubothrium sp. & Notothenia squamifrons & Kerguelen Subregion & Parukhin \& Lyadov (1982) \\
\hline 1777 & & & Eubothrium sp. & Notothenia squamifrons & Crozet Islands & Parukhin \& Lyadov (1982) \\
\hline 1778 & & & Eubothrium sp. & Notothenia squamifrons & Skif Bank & Parukhin \& Lyadov (1982) \\
\hline 1779 & & & Eubothrium sp. & Notothenia squamifrons & Ob Bank & Parukhin \& Lyadov (1982) \\
\hline 1780 & & & Eubothrium sp. & Notothenia squamifrons & Lena Bank & Parukhin \& Lyadov (1982) \\
\hline 1781 & & & Eubothrium sp. & Nototheniidae & Kerguelen Subregion & Parukhin \& Lyadov (1982) \\
\hline 1782 & \multirow{2}{*}{ ACANTHOCEPHALA } & Arhythmacanthidae & Heterosentis heteracanthus & blank & blank & Linstow (1896) \\
\hline 1783 & & & Heterosentis heteracanthus & Champsocephalus esox & $\begin{array}{c}\text { Beagle Channel } \\
\text { (Magellanic Sub-Region, }\end{array}$ & $\begin{array}{l}\text { Laskowski \& Zdzitowiecki } \\
\text { (2009) }\end{array}$ \\
\hline
\end{tabular}




\begin{tabular}{|c|c|c|c|c|c|c|}
\hline Index & Phylum & Family & Species & Host & Location & References \\
\hline & & & & & Sub-Antarctic) & \\
\hline 1784 & & & Heterosentis heteracanthus & Dissostichus eleginoides & Shag Rocks & Brickle et al. (2005) \\
\hline 1785 & & & Heterosentis heteracanthus & Lepidonotothen squamifrons & South Georgia Island & Zdzitowiecki (1990c) \\
\hline 1786 & & & Heterosentis heteracanthus & Lindbergichthys nudifrons & South Georgia Island & Zdzitowiecki (1990c) \\
\hline 1787 & & & Heterosentis heteracanthus & Muraenolepis microps & South Shetland Islands & Palm et al. (2007) \\
\hline 1788 & & & Heterosentis heteracanthus & Notothenia coriiceps & $\begin{array}{c}\text { Potter Cove (King George } \\
\text { Island) }\end{array}$ & Palm et.al. (1998) \\
\hline 1789 & & & Heterosentis heteracanthus & Notothenia coriiceps & King George Island & Palm et.al. (1998) \\
\hline 1790 & & & Heterosentis heteracanthus & Notothenia coriiceps & Admiralty Bay & $\begin{array}{l}\text { Zdzitowiecki \& Laskowski } \\
\text { (2004) }\end{array}$ \\
\hline 1791 & \multirow{16}{*}{ ACANTHOCEPHALA } & Arhythmacanthidae & Heterosentis heteracanthus & Notothenia rossii & South Georgia Island & Zdzitowiecki (1990c) \\
\hline 1792 & & & Heterosentis heteracanthus & Parachaenichthys georgianus & Grytviken (South Georgia) & Zdzitowiecki (1987) \\
\hline 1793 & & & Heterosentis heteracanthus & Parachaenichthys georgianus & South Georgia Island & Zdzitowiecki (1990c) \\
\hline 1794 & & & Heterosentis heteracanthus & Patagonotothen longipes & $\begin{array}{c}\text { Beagle Channel } \\
\text { (Magellanic Sub-Region, } \\
\text { Sub-Antarctic) }\end{array}$ & $\begin{array}{l}\text { Laskowski \& Zdzitowiecki } \\
\text { (2009) }\end{array}$ \\
\hline 1795 & & & Heterosentis heteracanthus & Patagonotothen tessellata & $\begin{array}{l}\text { Beagle Channel } \\
\text { (Magellanic Sub-Region, } \\
\text { Sub-Antarctic) }\end{array}$ & $\begin{array}{l}\text { Laskowski \& Zdzitowiecki } \\
\text { (2009) }\end{array}$ \\
\hline 1796 & & & Hypoechinorhynchus magellanicus & blank & blank & Szidat (1950) \\
\hline 1797 & & & Hypoechinorhynchus magellanicus & Champsocephalus esox & $\begin{array}{c}\text { Beagle Channel } \\
\text { (Magellanic Sub-Region, } \\
\text { Sub-Antarctic) }\end{array}$ & $\begin{array}{l}\text { Laskowski \& Zdzitowiecki } \\
\text { (2009) }\end{array}$ \\
\hline 1798 & & & Hypoechinorhynchus magellanicus & Champsocephalus esox & $\begin{array}{c}\text { Beagle Channel } \\
\text { (Magellanic Subregion) }\end{array}$ & $\begin{array}{l}\text { Laskowski \& Zdzitowiecki } \\
\qquad(2008)\end{array}$ \\
\hline 1799 & & & Hypoechinorhynchus magellanicus & Lepidonotothen squamifrons & Lena Bank & Parukhin (1986) \\
\hline 1800 & & & Hypoechinorhynchus magellanicus & Notothenia coriiceps & $\begin{array}{l}\text { Bhaia Scotia La Isla } \\
\text { Laurie (Orcadas del sur, } \\
\text { Argentina) }\end{array}$ & Szidat (1965) \\
\hline 1801 & & & Hypoechinorhynchus magellanicus & Notothenia coriiceps & Isle Laurie & Szidat (1965) \\
\hline 1802 & & & Hypoechinorhynchus magellanicus & Notothenia coriiceps & Orcadas Del Sur & Szidat (1965) \\
\hline 1803 & & & Hypoechinorhynchus magellanicus & Notothenia coriiceps & Archipelago Melcihor & Szidat (1965) \\
\hline 1804 & & & Hypoechinorhynchus magellanicus & Notothenia rossii & Lena Bank & Parukhin (1986) \\
\hline 1805 & & & Hypoechinorhynchus magellanicus & Parachaenichthys charcoti & South Shetland Islands & Szidat \& Graefe (1967) \\
\hline 1806 & & & Hypoechinorhynchus magellanicus & HOST NAME NOT MENTIONED & Subantarctic Region & Zdzitowiecki (1990b) \\
\hline
\end{tabular}




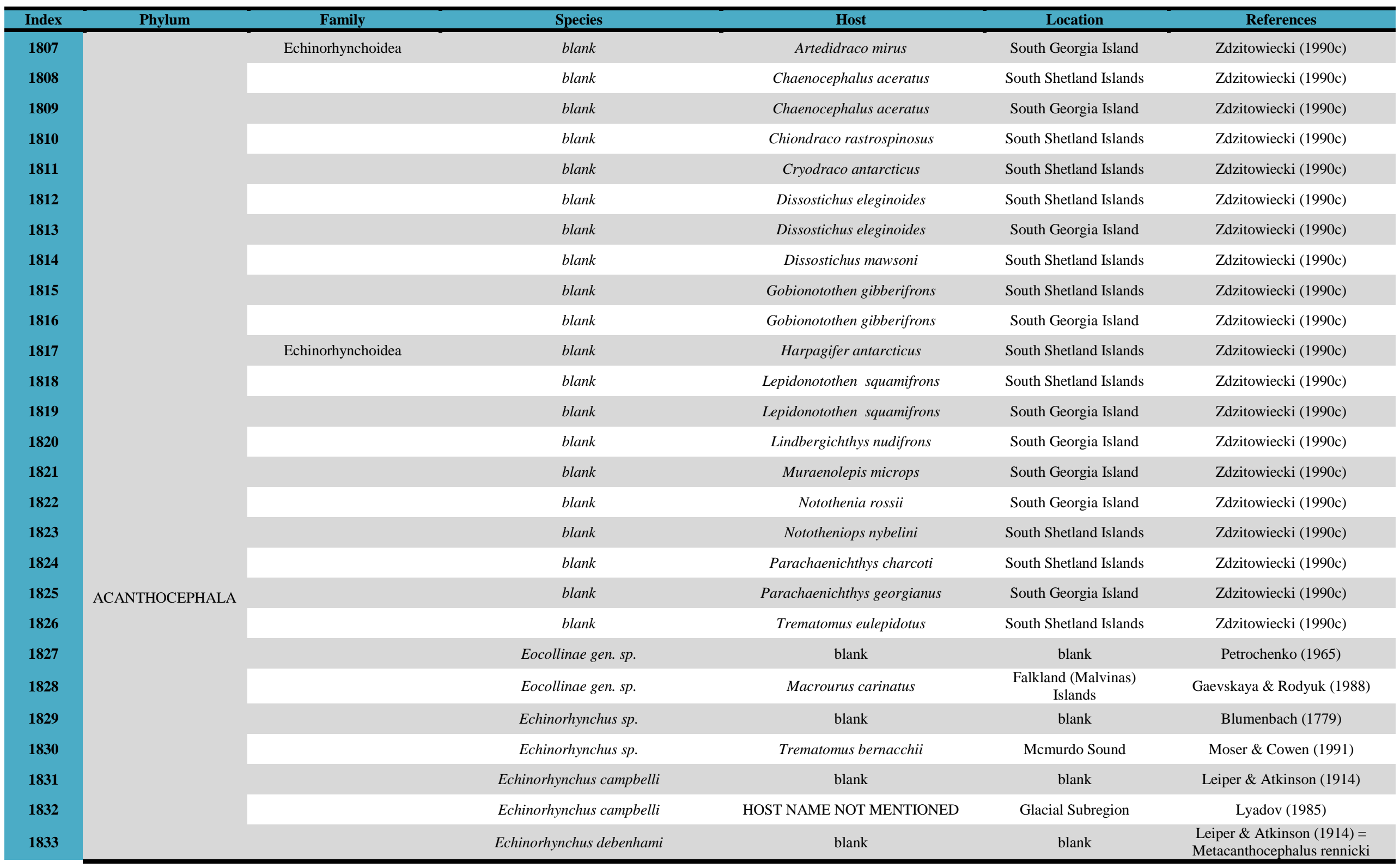




\begin{tabular}{|c|c|c|c|c|c|c|}
\hline Index & Phylum & Family & Species & Host & Location & References \\
\hline 1834 & & & Echinorhynchus debenhami & Champsocephalus gunnari & Kerguelen Subregion & Parukhin \& Lyadov (1981) \\
\hline 1835 & & & Echinorhynchus debenhami & Dissostichus eleginoides & Kerguelen Subregion & Parukhin \& Lyadov (1981) \\
\hline 1836 & & & Echinorhynchus debenhami & Dissostichus eleginoides & Crozet Islands & Parukhin \& Lyadov (1981) \\
\hline 1837 & & & Echinorhynchus debenhami & Dissostichus eleginoides & Ob Bank & Parukhin \& Lyadov (1981) \\
\hline 1838 & & & Echinorhynchus debenhami & Dissostichus eleginoides & Kerguelen Subregion & Parukhin \& Lyadov (1982) \\
\hline 1839 & & & Echinorhynchus debenhami & Dissostichus eleginoides & Skif Bank & Parukhin \& Lyadov (1982) \\
\hline 1840 & & & Echinorhynchus debenhami & Dissostichus eleginoides & Lena Bank & Parukhin (1986) \\
\hline 1841 & & & Echinorhynchus debenhami & Lepidonotothen squamifrons & Kerguelen Subregion & Parukhin \& Lyadov (1982) \\
\hline 1842 & & & Echinorhynchus debenhami & Lepidonotothen squamifrons & Crozet Islands & Parukhin \& Lyadov (1982) \\
\hline 1843 & & & Echinorhynchus debenhami & Lepidonotothen squamifrons & Kerguelen Subregion & Parukhin \& Lyadov (1981) \\
\hline 1844 & & & Echinorhynchus debenhami & Lepidonotothen squamifrons & Ob Bank & Parukhin \& Lyadov (1981) \\
\hline 1845 & & & Echinorhynchus debenhami & Lepidonotothen squamifrons & Lena Bank & Parukhin \& Lyadov (1981) \\
\hline 1846 & \multirow{14}{*}{ ACANTHOCEPHALA } & Echinorhynchoidea & Echinorhynchus debenhami & Lepidonotothen mizops & Kerguelen Subregion & Parukhin \& Lyadov (1981) \\
\hline 1847 & & & Echinorhynchus debenhami & blank & blank & Parukhin \& Lyadov (1982) \\
\hline 1848 & & & Echinorhynchus debenhami & Notothenia squamifrons & Crozet Islands & Parukhin \& Lyadov (1981) \\
\hline 1849 & & & Echinorhynchus debenhami & Nototheniidae & Kerguelen Subregion & Parukhin \& Lyadov (1982) \\
\hline 1850 & & & Echinorhynchus debenhami & Trematomus eulepidotus & Weddell Sea & Kock et al. (1984) \\
\hline 1851 & & & Echinorhynchus debenhami & HOST NAME NOT MENTIONED & Glacial Subregion & Lyadov (1985) \\
\hline 1852 & & & Echinorhynchus debenhami & HOST NAME NOT MENTIONED & Kerguelen Subregion & Lyadov (1985) \\
\hline 1853 & & & Echinorhynchus georgianus & blank & blank & $\begin{array}{c}\text { Rodjuk }(1986)=\mathrm{E} . \\
\text { petrotschenkoi }\end{array}$ \\
\hline 1854 & & & Echinorhynchus georgianus & Dissostichus eleginoides & Falkland-Patagonian Shelf & Gaevskaya et al. (1990) \\
\hline 1855 & & & Echinorhynchus longiproboscis & blank & blank & Rodjuk (1986) \\
\hline 1856 & & & Echinorhynchus longiproboscis & Dissostichus eleginoides & Falkland-Patagonian Shelf & Gaevskaya et al. (1990) \\
\hline 1857 & & & Echinorhynchus longiproboscis & Dissostichus eleginoides & Falkland Islands & Brickle et al. (2006) \\
\hline 1858 & & & Echinorhynchus longiproboscis & Macrourus carinatus & $\begin{array}{l}\text { Falkland (Malvinas) } \\
\text { Islands }\end{array}$ & Gaevskaya \& Rodyuk (1988) \\
\hline 1859 & & & Echinorhynchus nototheniae & blank & blank & $\begin{array}{c}\text { Zdzitowiecki }(1986)=E . \\
\text { petrotschenkoi }\end{array}$ \\
\hline
\end{tabular}




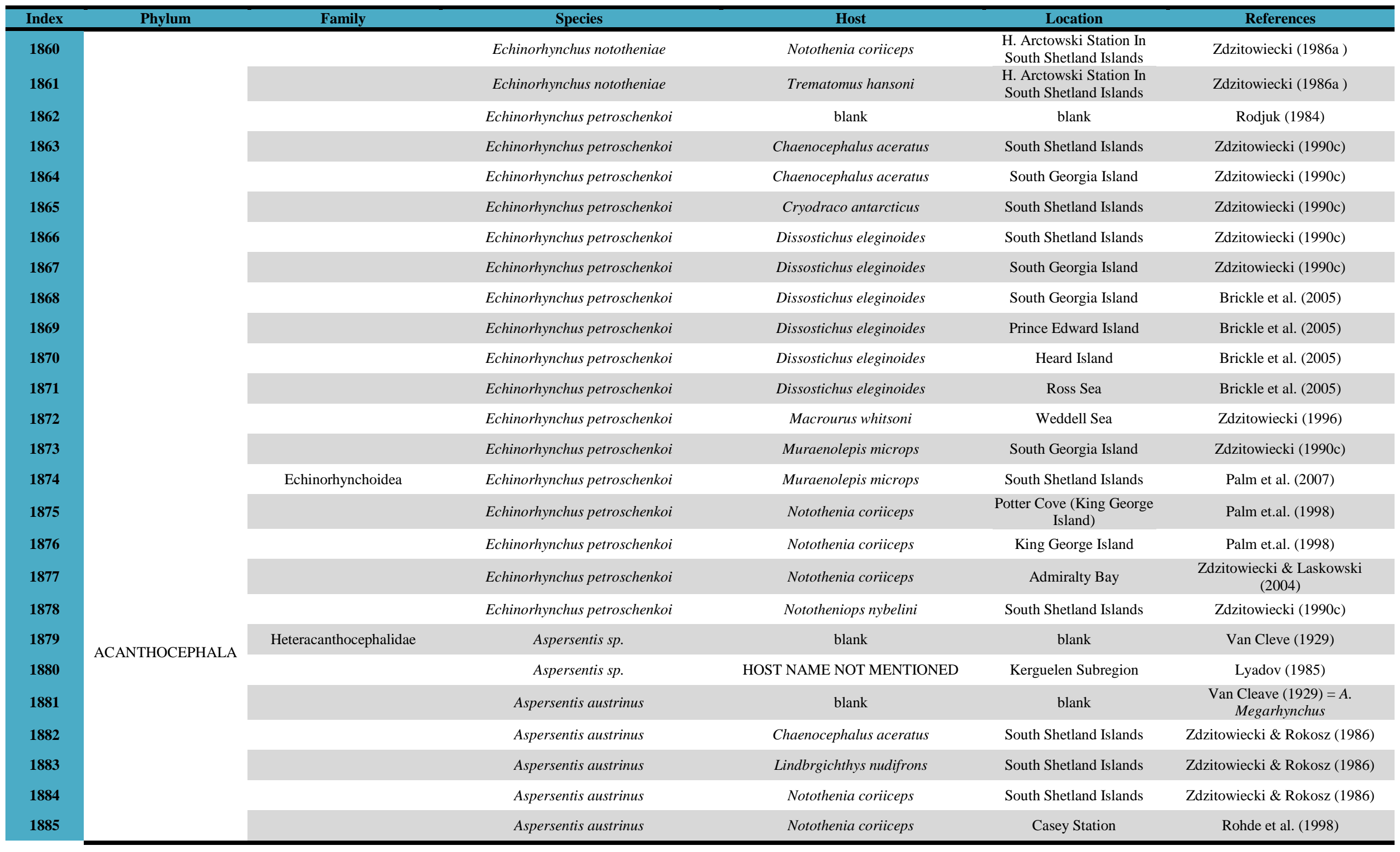




\begin{tabular}{|c|c|c|c|c|c|c|}
\hline Index & Phylum & Family & Species & Host & Location & References \\
\hline 1886 & & & Aspersentis austrinus & Notothenia gibberifrons & South Shetland Islands & Zdzitowiecki \& Rokosz (1986) \\
\hline 1887 & & & Aspersentis austrinus & Notothenia rossii & South Shetland Islands & Zdzitowiecki \& Rokosz (1986) \\
\hline 1888 & & & Aspersentis austrinus & Parachaenichthys charcoti & South Shetland Islands & Zdzitowiecki \& Rokosz (1986) \\
\hline 1889 & & & Aspersentis austrinus & Parachaenichthys georgianus & Grytviken (South Georgia) & Zdzitowiecki (1987) \\
\hline 1890 & & & Aspersentis austrinus & Trematomus newnesi & South Shetland Islands & Zdzitowiecki \& Rokosz (1986) \\
\hline 1891 & & & Aspersentis austrinus & Trematomus bernacchii & South Shetland Islands & Zdzitowiecki \& Rokosz (1986) \\
\hline 1892 & & & Aspersentis austrinus & Trematomus hansoni & Grytviken (South Georgia) & Zdzitowiecki (1987) \\
\hline 1893 & & & Aspersentis austrinus & Trematomus hansoni & Casey Station & Rohde et al. (1998) \\
\hline 1894 & & & Aspersentis johni & blank & blank & Baylis (1929) \\
\hline 1895 & & & Aspersentis johni & Champsocephalus esox & $\begin{array}{c}\text { Beagle Channel } \\
\text { (Magellanic Sub-Region, } \\
\text { Sub-Antarctic) }\end{array}$ & $\begin{array}{c}\text { Laskowski \& Zdzitowiecki } \\
\text { (2009) }\end{array}$ \\
\hline 1896 & & & Aspersentis johni & Patagonotothen longipes & $\begin{array}{c}\text { Beagle Channel } \\
\text { (Magellanic Subregion) }\end{array}$ & $\begin{array}{c}\text { Laskowski \& Zdzitowiecki } \\
\text { (2004) }\end{array}$ \\
\hline 1897 & & & Aspersentis johni & Patagonotothen longipes & $\begin{array}{l}\text { Beagle Channel } \\
\text { (Magellanic Sub-Region, } \\
\text { Sub-Antarctic) }\end{array}$ & $\begin{array}{l}\text { Laskowski \& Zdzitowiecki } \\
\text { (2009) }\end{array}$ \\
\hline 1898 & & & Aspersentis megarhynchus & blank & blank & Linstow (1892) \\
\hline 1899 & & & Aspersentis megarhynchus & Chaenichthys rhinoceratus & Kerguelen Subregion & Parukhin \& Lyadov (1981) \\
\hline 1900 & & & Aspersentis megarhynchus & Dissostichus eleginoides & Prince Edward Island & Brickle et al. (2005) \\
\hline 1901 & & Heteracanthocephalidae & Aspersentis megarhynchus & Harpagifer antarcticus & South Shetland Islands & Zdzitowiecki (1990c) \\
\hline 1902 & & & Aspersentis megarhynchus & Harpagifer georgianus & Admiralty Bay & Zdzitowiecki \& Zadrozny (1999) \\
\hline 1903 & & & Aspersentis megarhynchus & Lepidonotothen squamifrons & Ob Bank & Parukhin (1986) \\
\hline 1904 & & & Aspersentis megarhynchus & Lepidonotothen squamifrons & South Shetland Islands & Palm et al. (2007) \\
\hline 1905 & & & Aspersentis megarhynchus & Muraenolepis microps & South Shetland Islands & Palm et al. (2007) \\
\hline 1906 & ACANTHOCEPHALA & & Aspersentis megarhynchus & Notothenia coriiceps & South Orkney Islands & Zdzitowiecki \& White (1996) \\
\hline 1907 & & & Aspersentis megarhynchus & Notothenia coriiceps & $\begin{array}{l}\text { Potter Cove (King George } \\
\text { Island) }\end{array}$ & Palm et.al. (1998) \\
\hline 1908 & & & Aspersentis megarhynchus & Notothenia coriiceps & King George Island & Palm et.al. (1998) \\
\hline 1909 & & & Aspersentis megarhynchus & Notothenia coriiceps & Admiralty Bay & $\begin{array}{c}\text { Zdzitowiecki \& Laskowski } \\
\text { (2004) }\end{array}$ \\
\hline 1910 & & & Aspersentis megarhynchus & Notothenia coriiceps & Vernadsky Station & $\begin{array}{c}\text { Zdzitowiecki \& Laskowski } \\
(2004)\end{array}$ \\
\hline
\end{tabular}




\begin{tabular}{|c|c|c|c|c|c|c|}
\hline Index & Phylum & Family & Species & Host & Location & References \\
\hline 1911 & & & Aspersentis megarhynchus & Notothenia rossii & Kerguelen Subregion & Parukhin \& Lyadov (1981) \\
\hline 1912 & & & Aspersentis megarhynchus & Notothenia rossii & Kherd Island & Parukhin \& Lyadov (1981) \\
\hline 1913 & & & Aspersentis megarhynchus & Notothenia rossii & Skif Bank & Parukhin \& Lyadov (1981) \\
\hline 1914 & & & Aspersentis megarhynchus & Notothenia rossii & Georgia & Parukhin \& Lyadov (1981) \\
\hline 1915 & & & Aspersentis megarhynchus & Notothenia rossii & Kerguelen Subregion & Parukhin \& Lyadov (1982) \\
\hline 1916 & & & Aspersentis megarhynchus & Notothenia rossii & Kherd Island & Parukhin \& Lyadov (1982) \\
\hline 1917 & & & Aspersentis megarhynchus & Notothenia rossii & Ob Bank & Parukhin \& Lyadov (1982) \\
\hline 1918 & & & Aspersentis megarhynchus & Notothenia rossii & Ob Bank & Parukhin (1986) \\
\hline 1919 & & & Aspersentis megarhynchus & Notothenia rossii & Lena Bank & Parukhin (1986) \\
\hline 1920 & & & Aspersentis megarhynchus & Notothenia rossii & South Shetland Islands & Zdzitowiecki (1990c) \\
\hline 1921 & & & Aspersentis megarhynchus & Nototheniidae & Kerguelen Subregion & Parukhin \& Lyadov (1982) \\
\hline 1922 & & & Aspersentis megarhynchus & Parachaenichthys charcoti & South Shetland Islands & Zdzitowiecki (1990c) \\
\hline 1923 & & & Aspersentis megarhynchus & Parachaenichthys charcoti & $\begin{array}{c}\text { Admiralty Bay (Off The } \\
\text { South Shetland Islands } \\
\text { Admiralty Bay Bransfield } \\
\text { Strait) }\end{array}$ & Zdzitowiecki (2001a) \\
\hline 1924 & & & Aspersentis megarhynchus & Parachaenichthys charcoti & South Shetland Islands & Palm et al. (2007) \\
\hline 1925 & & & Aspersentis megarhynchus & Trematomus bernacchii & $\begin{array}{l}\text { Port Foster (Deception } \\
\text { Island) }\end{array}$ & Ruhl et al. (2003) \\
\hline 1926 & & & Aspersentis megarhynchus & HOST NAME NOT MENTIONED & Glacial Subregion & Lyadov (1985) \\
\hline 1927 & & & Aspersentis megarhynchus & HOST NAME NOT MENTIONED & Kerguelen Subregion & Lyadov (1985) \\
\hline 1928 & \multirow{8}{*}{ ACANTHOCEPHALA } & Heteracanthocephalidae & Aspersentis megarhynchus & blank & blank & Linstow (1892) \\
\hline 1929 & & & Aspersentis megarhynchus & Chaenichthys rhinoceratus & Kerguelen Subregion & Lyadov et al. (1981) \\
\hline 1930 & & & Aspersentis megarhynchus & Notothenia magellanica & Ob Bank & Parukhin (1986) \\
\hline 1931 & & & Aspersentis zanclorhynchi & blank & blank & Johnston \& Best (1937) \\
\hline 1932 & & & Aspersentis zanclorhynchi & blank & blank & Smales (1996) \\
\hline 1933 & & & Aspersentis zanclorhynchi & Zanclorhynchus spinifer & Heard Island & Rohde et al. (1998) \\
\hline 1934 & & & Aspersentis zanclorhynchi & Zanclorhynchus spinifer & Macquarie Island & Rohde et al. (1998) \\
\hline 1935 & & & Heteracanthocephalus dissostichi & blank & blank & Parukhin (1986) \\
\hline
\end{tabular}




\begin{tabular}{|c|c|c|c|c|c|c|}
\hline Index & Phylum & Family & Species & Host & Location & References \\
\hline 1936 & & & Heteracanthocephalus dissostichi & Dissostichus eleginoides & Ob Bank & Parukhin (1986) \\
\hline 1937 & & & Aspersentis megarhynchus & Parachaenichthys charcoti & South Shetland Islands & Szidat \& Graefe (1967) \\
\hline 1938 & & & Aspersentis megarhynchus & Parachaenichthys charcoti & Subantarctic Region & Zdzitowiecki (1990b) \\
\hline 1939 & & Polymorphoidea & blank & Artedidraco mirus & South Georgia Island & Zdzitowiecki (1990c) \\
\hline 1940 & & & blank & Chaenocephalus aceratus & South Shetland Islands & Zdzitowiecki (1990c) \\
\hline 1941 & & & blank & Chaenocephalus aceratus & South Georgia Island & Zdzitowiecki (1990c) \\
\hline 1942 & & & blank & Chiondraco rastrospinosus & South Shetland Islands & Zdzitowiecki (1990c) \\
\hline 1943 & & & blank & Cryodraco antarcticus & South Shetland Islands & Zdzitowiecki (1990c) \\
\hline 1944 & & & blank & Dissostichus eleginoides & South Shetland Islands & Zdzitowiecki (1990c) \\
\hline 1945 & & & blank & Dissostichus eleginoides & South Georgia Island & Zdzitowiecki (1990c) \\
\hline 1946 & & & blank & Dissostichus mawsoni & South Shetland Islands & Zdzitowiecki (1990c) \\
\hline 1947 & & & blank & Gobionotothen gibberifrons & South Shetland Islands & Zdzitowiecki (1990c) \\
\hline 1948 & & & blank & Gobionotothen gibberifrons & South Georgia Island & Zdzitowiecki (1990c) \\
\hline 1949 & & & blank & Harpagifer antarcticus & South Shetland Islands & Zdzitowiecki (1990c) \\
\hline 1950 & & & blank & Lepidonotothen squamifrons & South Shetland Islands & Zdzitowiecki (1990c) \\
\hline 1951 & & & blank & Lepidonotothen squamifrons & South Georgia Island & Zdzitowiecki (1990c) \\
\hline 1952 & & & blank & Lindbergichthys nudifrons & South Georgia Island & Zdzitowiecki (1990c) \\
\hline 1953 & & & blank & Muraenolepis microps & South Georgia Island & Zdzitowiecki (1990c) \\
\hline 1954 & & & blank & Notothenia rossii & South Georgia Island & Zdzitowiecki (1990c) \\
\hline 1955 & & & blank & Nototheniops larseni & South Georgia Island & Zdzitowiecki (1990c) \\
\hline 1956 & & & blank & Nototheniops nybelini & South Shetland Islands & Zdzitowiecki (1990c) \\
\hline 1957 & \multirow{5}{*}{ ACANTHOCEPHALA } & Polymorphoidea & blank & Parachaenichthys charcoti & South Shetland Islands & Zdzitowiecki (1990c) \\
\hline 1958 & & & blank & Patagonotothen brevicauda guntheri & South Georgia Island & Zdzitowiecki (1990c) \\
\hline 1959 & & & Andracantha baylisi & blank & blank & Zdzitowiecki (1986) \\
\hline 1960 & & & Andracantha baylisi & Chaenocephalus aceratus & South Georgia Island & Zdzitowiecki (1990c) \\
\hline 1961 & & & Andracantha baylisi & Champsocephalus esox & $\begin{array}{c}\text { Eastern Mouth of Beagle } \\
\text { Channel (Magellanic } \\
\text { Subregion of Sub- } \\
\text { Antarctic Waters) }\end{array}$ & Laskowski et al. (2008) \\
\hline
\end{tabular}




\begin{tabular}{|c|c|c|c|c|c|c|}
\hline Index & Phylum & Family & Species & Host & Location & References \\
\hline 1962 & & & Andracantha baylisi & Champsocephalus esox & $\begin{array}{c}\text { Beagle Channel } \\
\text { (Magellanic Sub-Region, } \\
\text { Sub-Antarctic) }\end{array}$ & $\begin{array}{l}\text { Laskowski \& Zdzitowiecki } \\
\text { (2009) }\end{array}$ \\
\hline 1963 & & & Andracantha baylisi & Notothenia rossii & South Georgia Island & Zdzitowiecki (1990c) \\
\hline 1964 & & & Andracantha baylisi & Parachaenichthys georgianus & South Georgia Island & Zdzitowiecki (1990c) \\
\hline 1965 & & & Andracantha baylisi & Patagonotothen longipes & $\begin{array}{l}\text { Eastern Mouth of Beagle } \\
\text { Channel (Magellanic } \\
\text { Subregion of Sub- } \\
\text { Antarctic Waters) }\end{array}$ & Laskowski et al. (2008) \\
\hline 1966 & & & Andracantha baylisi & Patagonotothen longipes & $\begin{array}{c}\text { Beagle Channel } \\
\text { (Magellanic Sub-Region, } \\
\text { Sub-Antarctic) }\end{array}$ & $\begin{array}{l}\text { Laskowski \& Zdzitowiecki } \\
\text { (2009) }\end{array}$ \\
\hline 1967 & & & Corynosoma spp. (larvae) & blank & blank & Lühe (1904) \\
\hline 1968 & & & Corynosoma spp. (larvae) & Dissostichus eleginoides & Heard Island & Rohde et al. (1998) \\
\hline 1969 & & & Corynosoma spp. (larvae) & Gobionotothen acuta & Heard Island & Rohde et al. (1998) \\
\hline 1970 & & & Corynosoma spp. (larvae) & Lepidonotothen mizops & Heard Island & Rohde et al. (1998) \\
\hline 1971 & & & Corynosoma spp. (larvae) & Notothenia coriiceps & South Shetland Islands & Szidat \& Graefe (1967) \\
\hline 1972 & & & Corynosoma spp. (larvae) & Notothenia coriiceps & $\begin{array}{l}\text { Potter Cove (King George } \\
\text { Island) }\end{array}$ & Palm et.al. (1998) \\
\hline 1973 & & & Corynosoma spp. (larvae) & Notothenia coriiceps & King George Island & Palm et.al. (1998) \\
\hline 1974 & & & Corynosoma spp. & blank & blank & Lühe (1904) \\
\hline 1975 & & & Corynosoma spp. & Gobionotothen gibberifrons & South Shetland Islands & Palm et al. (2007) \\
\hline 1976 & & & Corynosoma spp. & Lepidonotothen nudifrons & South Shetland Islands & Palm et al. (2007) \\
\hline 1977 & & & Corynosoma spp. & Lepidonotothen squamifrons & South Shetland Islands & Palm et al. (2007) \\
\hline 1978 & & & Corynosoma spp. & Muraenolepis microps & South Shetland Islands & Palm et al. (2007) \\
\hline 1979 & & & Corynosoma spp. & Notothenia coriiceps & $\begin{array}{c}\text { Bhaia Scotia La Isla } \\
\text { Laurie (Orcadas del sur, } \\
\text { Argentina) }\end{array}$ & Szidat (1965) \\
\hline 1980 & & & Corynosoma spp. & Notothenia coriiceps & Isle Laurie & Szidat (1965) \\
\hline 1981 & \multirow{4}{*}{ ACANTHOCEPHALA } & Polymorphoidea & Corynosoma spp. & Notothenia coriiceps & Orcadas Del Sur & Szidat (1965) \\
\hline 1982 & & & Corynosoma spp. & Notothenia coriiceps & Archipelago Melcihor & Szidat (1965) \\
\hline 1983 & & & Corynosoma spp. & Nototheniops larseni & South Shetland Islands & Palm et al. (2007) \\
\hline 1984 & & & Corynosoma spp. & Parachaenichthys charcoti & South Shetland Islands & Palm et al. (2007) \\
\hline
\end{tabular}




\begin{tabular}{|c|c|c|c|c|c|c|}
\hline Index & Phylum & Family & Species & Host & Location & References \\
\hline 1985 & & & Corynosoma spp. & Patagonotothen tessellata & $\begin{array}{l}\text { Eastern Mouth of Beagle } \\
\text { Channel (Magellanic } \\
\text { Subregion of Sub- } \\
\text { Antarctic Waters) }\end{array}$ & Laskowski et al. (2008) \\
\hline 1986 & & & Corynosoma spp. & Patagonotothen tessellata & $\begin{array}{c}\text { Beagle Channel } \\
\text { (Magellanic Sub-Region, } \\
\text { Sub-Antarctic) }\end{array}$ & $\begin{array}{l}\text { Laskowski \& Zdzitowiecki } \\
\qquad(2009)\end{array}$ \\
\hline 1987 & & & Corynosoma spp. & Trematomus eulepidotus & South Shetland Islands & Palm et al. (2007) \\
\hline 1988 & & & Corynosoma arctocephali & blank & blank & Zdzitowiecki (1984) \\
\hline 1989 & & & Corynosoma arctocephali & Chaenocephalus aceratus & South Shetland Islands & Zdzitowiecki (1986b) \\
\hline 1990 & & & Corynosoma arctocephali & Chaenocephalus aceratus & South Shetland Islands & Palm et al. (2007) \\
\hline 1991 & & & Corynosoma arctocephali & Chaenocephalus aceratus & South Shetland Islands & Zdzitowiecki (1990c) \\
\hline 1992 & & & Corynosoma arctocephali & Chaenocephalus aceratus & South Georgia Island & Zdzitowiecki (1990c) \\
\hline 1993 & & & Corynosoma arctocephali & Chaenocephalus aceratus & South Orkney Islands & Zdzitowiecki \& White (1996) \\
\hline 1994 & & & Corynosoma arctocephali & Cryodraco antarcticus & South Shetland Islands & Zdzitowiecki (1990c) \\
\hline 1995 & & & Corynosoma arctocephali & Dissostichus eleginoides & South Georgia Island & Brickle et al. (2005) \\
\hline 1996 & & & Corynosoma arctocephali & Dissostichus eleginoides & Prince Edward Island & Brickle et al. (2005) \\
\hline 1997 & & & Corynosoma arctocephali & Dissostichus eleginoides & Heard Island & Brickle et al. (2005) \\
\hline 1998 & & & Corynosoma arctocephali & Dissostichus eleginoides & Macquarie Island & Brickle et al. (2005) \\
\hline 1999 & & & Corynosoma arctocephali & Dissostichus eleginoides & Ross Sea & Brickle et al. (2005) \\
\hline 2000 & & & Corynosoma arctocephali & Dissostichus eleginoides & South Shetland Islands & Zdzitowiecki (1990c) \\
\hline 2001 & & & Corynosoma arctocephali & Dissostichus eleginoides & South Georgia Island & Zdzitowiecki (1990c) \\
\hline 2002 & & & Corynosoma arctocephali & Gobionotothen gibberifrons & South Orkney Islands & Zdzitowiecki \& White (1996) \\
\hline 2003 & & & Corynosoma arctocephali & Harpagifer antarcticus & Vernadsky Station & $\begin{array}{c}\text { Laskowski \& Zdzitowiecki } \\
\text { (2005) }\end{array}$ \\
\hline 2004 & & & Corynosoma arctocephali & Harpagifer georgianus & Admiralty Bay & Zdzitowiecki \& Zadrozny (1999) \\
\hline 2005 & & & Corynosoma arctocephali & Lepidonotothen larseni & $\begin{array}{l}\text { Port Foster (Deception } \\
\text { Island) }\end{array}$ & Ruhl et al. (2003) \\
\hline 2006 & & & Corynosoma arctocephali & Lepidonotothen nudifrons & $\begin{array}{l}\text { Port Foster (Deception } \\
\text { Island) }\end{array}$ & Ruhl et al. (2003) \\
\hline 2007 & & & Corynosoma arctocephali & Lepidonotothen squamifrons & South Shetland Islands & Palm et al. (2007) \\
\hline 2008 & ACANTHOCEPHALA & Polymorphoidea & Corynosoma arctocephali & Lepidonotothen squamifrons & South Shetland Islands & Zdzitowiecki (1990c) \\
\hline
\end{tabular}




\begin{tabular}{|c|c|c|c|c|c|c|}
\hline Index & Phylum & Family & Species & Host & Location & References \\
\hline 2009 & & & Corynosoma arctocephali & Lepidonotothen squamifrons & South Georgia Island & Zdzitowiecki (1990c) \\
\hline 2010 & & & Corynosoma arctocephali & Lindbergichthys nudifrons & Vernadsky Station & $\begin{array}{c}\text { Laskowski \& Zdzitowiecki } \\
\text { (2005) }\end{array}$ \\
\hline 2011 & & & Corynosoma arctocephali & Muraenolepis microps & South Georgia Island & Zdzitowiecki (1990c) \\
\hline 2012 & & & Corynosoma arctocephali & Notothenia coriiceps & South Shetland Islands & Zdzitowiecki (1986b) \\
\hline 2013 & & & Corynosoma arctocephali & Notothenia coriiceps & Admiralty Bay & $\begin{array}{l}\text { Zdzitowiecki \& Laskowski } \\
\text { (2004) }\end{array}$ \\
\hline 2014 & & & Corynosoma arctocephali & Notothenia coriiceps & Vernadsky Station & $\begin{array}{l}\text { Zdzitowiecki \& Laskowski } \\
\text { (2004) }\end{array}$ \\
\hline 2015 & & & Corynosoma arctocephali & Notothenia coriiceps & South Orkney Islands & Zdzitowiecki \& White (1996) \\
\hline 2016 & & & Corynosoma arctocephali & Notothenia rossi marmorata & South Shetland Islands & Zdzitowiecki (1986b) \\
\hline 2017 & & & Corynosoma arctocephali & Notothenia rossii & South Georgia Island & Zdzitowiecki (1990c) \\
\hline 2018 & & & Corynosoma arctocephali & Nototheniops larseni & South Georgia Island & Zdzitowiecki (1990c) \\
\hline 2019 & & & Corynosoma arctocephali & Parachaenichthys charcoti & South Shetland Islands & Zdzitowiecki (1986b) \\
\hline 2020 & & & Corynosoma arctocephali & Parachaenichthys charcoti & Admiralty Bay & Zdzitowiecki (2001a) \\
\hline 2021 & & & Corynosoma arctocephali & Parachaenichthys georgianus & South Georgia Island & Zdzitowiecki (1990c) \\
\hline 2022 & & & Corynosoma arctocephali & Patagonotothen brevicauda guntheri & South Georgia Island & Zdzitowiecki (1990c) \\
\hline 2023 & & & Corynosoma arctocephali & Patagonotothen brevicaudata guntheri & South Georgia Island & Zdzitowiecki (1990c) \\
\hline 2024 & & & Corynosoma arctocephali & Trematomus bernacchii & $\begin{array}{l}\text { Port Foster (Deception } \\
\text { Island) }\end{array}$ & Ruhl et al. (2003) \\
\hline 2025 & & & Corynosoma arctocephali & Trematomus bernacchii & Vernadsky Station & $\begin{array}{c}\text { Laskowski \& Zdzitowiecki } \\
\text { (2005) }\end{array}$ \\
\hline 2026 & & & Corynosoma beaglense & blank & blank & $\begin{array}{c}\text { Laskowski, Jesewski \& } \\
\text { Zdzitowiecki (2008) }\end{array}$ \\
\hline 2027 & & & Corynosoma beaglense & Champsocephalus esox & $\begin{array}{l}\text { Eastern Mouth of Beagle } \\
\text { Channel (Magellanic } \\
\text { Subregion of Sub- } \\
\text { Antarctic Waters) }\end{array}$ & Laskowski et al. (2008) \\
\hline 2028 & & & Corynosoma beaglense & Champsocephalus esox & $\begin{array}{c}\text { Beagle Channel } \\
\text { (Magellanic Sub-Region, } \\
\text { Sub-Antarctic) }\end{array}$ & $\begin{array}{l}\text { Laskowski \& Zdzitowiecki } \\
\qquad(2009)\end{array}$ \\
\hline 2029 & & & Corynosoma bullosum & blank & blank & Linstow (1892) \\
\hline 2030 & & & Corynosoma bullosum & Artedidraco loennbergi & Weddell Sea & Zdzitowiecki (1996) \\
\hline 2031 & & & Corynosoma bullosum & Artedidraco mirus & South Georgia Island & Zdzitowiecki (1990c) \\
\hline 2032 & & & Corynosoma bullosum & Artedidraco orianae & Weddell Sea & Zdzitowiecki (1996) \\
\hline
\end{tabular}




\begin{tabular}{|c|c|c|c|c|c|c|}
\hline Index & Phylum & Family & Species & Host & Location & References \\
\hline 2033 & \multirow{27}{*}{ ACANTHOCEPHALA } & & Corynosoma bullosum & Chaenocephalus aceratus & South Shetland Islands & Zdzitowiecki (1986b) \\
\hline 2034 & & Polymorphoidea & Corynosoma bullosum & Chaenocephalus aceratus & South Shetland Islands & Zdzitowiecki (1990c) \\
\hline 2035 & & & Corynosoma bullosum & Chaenocephalus aceratus & South Georgia Island & Zdzitowiecki (1990c) \\
\hline 2036 & & & Corynosoma bullosum & Chaenocephalus aceratus & South Orkney Islands & Zdzitowiecki \& White (1996) \\
\hline 2037 & & & Corynosoma bullosum & Chaenocephalus aceratus & South Shetland Islands & Palm et al. (2007) \\
\hline 2038 & & & Corynosoma bullosum & Chionodraco rastrospinosus & South Shetland Islands & Zdzitowiecki (1986b) \\
\hline 2039 & & & Corynosoma bullosum & blank & blank & Zdzitowiecki (1990c) \\
\hline 2040 & & & Corynosoma bullosum & Cryodraco antarcticus & South Shetland Islands & Zdzitowiecki (1986b) \\
\hline 2041 & & & Corynosoma bullosum & blank & blank & Zdzitowiecki (1990c) \\
\hline 2042 & & & Corynosoma bullosum & Dissostichus eleginoides & South Shetland Islands & Zdzitowiecki (1986b) \\
\hline 2043 & & & Corynosoma bullosum & Dissostichus eleginoides & South Georgia Island & Gaevskaya et al. (1990) \\
\hline 2044 & & & Corynosoma bullosum & Dissostichus eleginoides & South Shetland Islands & Zdzitowiecki (1990c) \\
\hline 2045 & & & Corynosoma bullosum & Dissostichus eleginoides & South Georgia Island & Zdzitowiecki (1990c) \\
\hline 2046 & & & Corynosoma bullosum & Dissostichus eleginoides & Shag Rocks & Brickle et al. (2005) \\
\hline 2047 & & & Corynosoma bullosum & Dissostichus eleginoides & South Georgia Island & Brickle et al. (2005) \\
\hline 2048 & & & Corynosoma bullosum & Dissostichus eleginoides & Prince Edward Island & Brickle et al. (2005) \\
\hline 2049 & & & Corynosoma bullosum & Dissostichus eleginoides & Heard Island & Brickle et al. (2005) \\
\hline 2050 & & & Corynosoma bullosum & Dissostichus eleginoides & Macquarie Island & Brickle et al. (2005) \\
\hline 2051 & & & Corynosoma bullosum & Dissostichus eleginoides & Ross Sea & Brickle et al. (2005) \\
\hline 2052 & & & Corynosoma bullosum & Dissostichus eleginoides & Falkland Islands & Brickle et al. (2006) \\
\hline 2053 & & & Corynosoma bullosum & Dissostichus mawsoni & South Shetland Islands & Zdzitowiecki (1990c) \\
\hline 2054 & & & Corynosoma bullosum & Gobionotothen gibberifrons & South Orkney Islands & Zdzitowiecki \& White (1996) \\
\hline 2055 & & & Corynosoma bullosum & Gobionotothen gibberifrons & South Shetland Islands & Palm et al. (2007) \\
\hline 2056 & & & Corynosoma bullosum & Harpagiferidae gen. sp. & South Shetland Islands & Zdzitowiecki (1986b) \\
\hline 2057 & & & Corynosoma bullosum & Lepidonotothen squamifrons & South Shetland Islands & Zdzitowiecki (1990c) \\
\hline 2058 & & & Corynosoma bullosum & Lepidonotothen squamifrons & South Georgia Island & Zdzitowiecki (1990c) \\
\hline 2059 & & & Corynosoma bullosum & Lepidonotothen squamifrons & South Shetland Islands & Palm et al. (2007) \\
\hline
\end{tabular}




\begin{tabular}{|c|c|c|c|c|c|c|}
\hline Index & Phylum & Family & Species & Host & Location & References \\
\hline 2060 & & & Corynosoma bullosum & Lindbergichthys nudifrons & South Shetland Islands & Zdzitowiecki (1986b) \\
\hline 2061 & & & Corynosoma bullosum & Lindbergichthys nudifrons & South Georgia Island & Zdzitowiecki (1990c) \\
\hline 2062 & & & Corynosoma bullosum & Lindbergichthys nudifrons & South Shetland Islands & Palm et al. (2007) \\
\hline 2063 & \multirow{23}{*}{ ACANTHOCEPHALA } & Polymorphoidea & Corynosoma bullosum & Lycodichthys antarcticus & Weddell Sea & Zdzitowiecki (1996) \\
\hline 2064 & & & Corynosoma bullosum & Macrourus whitsoni & Weddell Sea & Zdzitowiecki (1996) \\
\hline 2065 & & & Corynosoma bullosum & Muraenolepis microps & South Georgia Island & Zdzitowiecki (1990c) \\
\hline 2066 & & & Corynosoma bullosum & Muraenolepis microps & South Shetland Islands & Palm et al. (2007) \\
\hline 2067 & & & Corynosoma bullosum & Notothenia coriiceps & South Shetland Islands & Zdzitowiecki (1986b) \\
\hline 2068 & & & Corynosoma bullosum & Notothenia coriiceps & South Orkney Islands & Zdzitowiecki \& White (1996) \\
\hline 2069 & & & Corynosoma bullosum & Notothenia coriiceps & Admiralty Bay & $\begin{array}{c}\text { Zdzitowiecki \& Laskowski } \\
\text { (2004) }\end{array}$ \\
\hline 2070 & & & Corynosoma bullosum & Notothenia coriiceps & Vernadsky Station & $\begin{array}{c}\text { Zdzitowiecki \& Laskowski } \\
\text { (2004) }\end{array}$ \\
\hline 2071 & & & Corynosoma bullosum & Notothenia gibberifrons & South Shetland Islands & Zdzitowiecki (1986b) \\
\hline 2072 & & & Corynosoma bullosum & Notothenia gibberifrons & South Shetland Islands & Zdzitowiecki (1990c) \\
\hline 2073 & & & Corynosoma bullosum & Notothenia gibberifrons & South Georgia Island & Zdzitowiecki (1990c) \\
\hline 2074 & & & Corynosoma bullosum & Notothenia rossii & South Shetland Islands & Zdzitowiecki (1986b) \\
\hline 2075 & & & Corynosoma bullosum & Notothenia rossii & South Georgia Island & Zdzitowiecki (1990c) \\
\hline 2076 & & & Corynosoma bullosum & Nototheniops larseni & South Georgia Island & Zdzitowiecki (1990c) \\
\hline 2077 & & & Corynosoma bullosum & Nototheniops larseni & South Shetland Islands & Palm et al. (2007) \\
\hline 2078 & & & Corynosoma bullosum & Nototheniops nybelini & South Shetland Islands & Zdzitowiecki (1990c) \\
\hline 2079 & & & Corynosoma bullosum & Parachaenichthys charcoti & South Shetland Islands & Zdzitowiecki (1986b) \\
\hline 2080 & & & Corynosoma bullosum & Parachaenichthys charcoti & South Shetland Islands & Zdzitowiecki (1990c) \\
\hline 2081 & & & Corynosoma bullosum & Parachaenichthys charcoti & Admiralty Bay & Zdzitowiecki (2001a) \\
\hline 2082 & & & Corynosoma bullosum & Parachaenichthys charcoti & $\begin{array}{l}\text { Off the South Shetland } \\
\text { Islands }\end{array}$ & Zdzitowiecki (2001a) \\
\hline 2083 & & & Corynosoma bullosum & Parachaenichthys charcoti & $\begin{array}{c}\text { Admiralty Bay Bransfield } \\
\text { Strait }\end{array}$ & Zdzitowiecki (2001a) \\
\hline 2084 & & & Corynosoma bullosum & Parachaenichthys charcoti & South Shetland Islands & Palm et al. (2007) \\
\hline 2085 & & & Corynosoma bullosum & Parachaenichthys georgianus & Grytviken (South Georgia) & Zdzitowiecki (1987) \\
\hline
\end{tabular}




\begin{tabular}{|c|c|c|c|c|c|c|}
\hline Index & Phylum & Family & Species & Host & Location & References \\
\hline 2086 & & & Corynosoma bullosum & Parachaenichthys georgianus & South Georgia Island & Zdzitowiecki (1990c) \\
\hline 2087 & & & Corynosoma bullosum & Paraliparis $s p$. & South Shetland Islands & Zdzitowiecki (1986b) \\
\hline 2088 & & & Corynosoma bullosum & Patagonotothen brevicauda guntheri & South Georgia Island & Zdzitowiecki (1990c) \\
\hline 2089 & & & Corynosoma bullosum & Pogonophryne permitini & Weddell Sea & Zdzitowiecki (1996) \\
\hline 2090 & & & Corynosoma bullosum & Prionodraco evansii & Weddell Sea & Zdzitowiecki (1996) \\
\hline 2091 & & & Corynosoma bullosum & Pseudochaenichthys georginanus & South Shetland Islands & Zdzitowiecki (1990c) \\
\hline 2092 & \multirow{18}{*}{ ACANTHOCEPHALA } & Polymorphoidea & Corynosoma bullosum & Pseudochaenichthys georginanus & South Georgia Island & Zdzitowiecki (1990c) \\
\hline 2093 & & & Corynosoma bullosum & Racovitzia glacialis & Weddell Sea & Zdzitowiecki (1996) \\
\hline 2094 & & & Corynosoma bullosum & Trematomus bernacchii & South Shetland Islands & Zdzitowiecki (1986b) \\
\hline 2095 & & & Corynosoma bullosum & Trematomus eulepidotus & Weddell Sea & Zdzitowiecki (1996) \\
\hline 2096 & & & Corynosoma bullosum & Trematomus eulepidotus & South Shetland Islands & Palm et al. (2007) \\
\hline 2097 & & & Corynosoma bullosum & Trematomus hansoni & South Shetland Islands & Zdzitowiecki (1986b) \\
\hline 2098 & & & Corynosoma bullosum & Trematomus hansoni & Grytviken (South Georgia) & Zdzitowiecki (1987) \\
\hline 2099 & & & Corynosoma bullosum & Trematomus hansoni & Weddell Sea & Zdzitowiecki (1996) \\
\hline 2100 & & & Corynosoma bullosum & Trematomus lepidorhinus & Weddell Sea & Zdzitowiecki (1996) \\
\hline 2101 & & & Corynosoma bullosum & Trematomus loennbergi & Weddell Sea & Zdzitowiecki (1996) \\
\hline 2102 & & & Corynosoma bullosum & Trematomus pennelli & Weddell Sea & Zdzitowiecki (1996) \\
\hline 2103 & & & Corynosoma bullosum & Trematomus scotti & Weddell Sea & Zdzitowiecki (1996) \\
\hline 2104 & & & Corynosoma evae & blank & blank & Zdzitowiecki (1984) \\
\hline 2105 & & & Corynosoma evae & Champsocephalus esox & $\begin{array}{l}\text { Eastern Mouth of Beagle } \\
\text { Channel \& Near Ushuaia }\end{array}$ & Laskowski et al. (2008) \\
\hline 2106 & & & Corynosoma evae & Champsocephalus esox & $\begin{array}{c}\text { Terra Del Fuego } \\
\text { (Magellanic Subregion of } \\
\text { Sub-Antarctic Waters) }\end{array}$ & Laskowski et al. (2008) \\
\hline 2107 & & & Corynosoma evae & Champsocephalus esox & $\begin{array}{c}\text { Beagle Channel } \\
\text { (Magellanic Sub-Region, } \\
\text { Sub-Antarctic) }\end{array}$ & $\begin{array}{l}\text { Laskowski \& Zdzitowiecki } \\
\text { (2009) }\end{array}$ \\
\hline 2108 & & & Corynosoma evae & Patagonotothen longipes & $\begin{array}{l}\text { Eastern Mouth of Beagle } \\
\text { Channel \& Near Ushuaia }\end{array}$ & Laskowski et al. (2008) \\
\hline 2109 & & & Corynosoma evae & Patagonotothen longipes & $\begin{array}{c}\text { Terra Del Fuego } \\
\text { (Magellanic Subregion of } \\
\text { Sub-Antarctic Waters) }\end{array}$ & Laskowski et al. (2008) \\
\hline
\end{tabular}









\begin{tabular}{|c|c|c|c|c|c|c|}
\hline Index & Phylum & Family & Species & Host & Location & References \\
\hline 2136 & & & Corynosoma hamanni & Dissostichus eleginoides & Kerguelen Subregion & Parukhin \& Lyadov (1982) \\
\hline 2137 & & & Corynosoma hamanni & Dissostichus eleginoides & Skif Bank & Parukhin \& Lyadov (1982) \\
\hline 2138 & & & Corynosoma hamanni & Dissostichus eleginoides & Ob Bank & Parukhin \& Lyadov (1982) \\
\hline 2139 & & & Corynosoma hamanni & Dissostichus eleginoides & Lena Bank & Parukhin \& Lyadov (1982) \\
\hline 2140 & & & Corynosoma hamanni & Dissostichus eleginoides & Ob Bank & Parukhin (1986) \\
\hline 2141 & & & Corynosoma hamanni & Dissostichus eleginoides & Lena Bank & Parukhin (1986) \\
\hline 2142 & & & Corynosoma hamanni & Dissostichus mawsoni & South Shetland Islands & Zdzitowiecki (1990c) \\
\hline 2143 & & & Corynosoma hamanni & Gobionotothen acuta & Kerguelen Subregion & Parukhin \& Lyadov (1981) \\
\hline 2144 & & & Corynosoma hamanni & blank & blank & Parukhin \& Lyadov (1982) \\
\hline 2145 & & & Corynosoma hamanni & Gobionotothen gibberifrons & South Orkney Islands & Zdzitowiecki \& White (1996) \\
\hline 2146 & & & Corynosoma hamanni & Harpagifer antarcticus & South Shetland Islands & Zdzitowiecki (1990c) \\
\hline 2147 & \multirow{16}{*}{ ACANTHOCEPHALA } & Polymorphoidea & Corynosoma hamanni & Harpagifer georgianus & Off Elephant Island & Zdzitowiecki \& Zadrozny (1999) \\
\hline 2148 & & & Corynosoma hamanni & Lepidonotothen larseni & $\begin{array}{l}\text { Port Foster (Deception } \\
\text { Island) }\end{array}$ & Ruhl et al. (2003) \\
\hline 2149 & & & Corynosoma hamanni & Lepidonotothen mizops & Kerguelen Subregion & Parukhin \& Lyadov (1981) \\
\hline 2150 & & & Corynosoma hamanni & blank & blank & Parukhin \& Lyadov (1982) \\
\hline 2151 & & & Corynosoma hamanni & Lindbergichthys nudifrons & South Shetland Islands & Zdzitowiecki (1986b) \\
\hline 2152 & & & Corynosoma hamanni & Lepidonotothen nudifrons & $\begin{array}{l}\text { Port Foster (Deception } \\
\text { Island) }\end{array}$ & Ruhl et al. (2003) \\
\hline 2153 & & & Corynosoma hamanni & Lepidonotothen squamifrons & Kerguelen Subregion & Parukhin \& Lyadov (1981) \\
\hline 2154 & & & Corynosoma hamanni & Lepidonotothen squamifrons & Crozet Islands & Parukhin \& Lyadov (1981) \\
\hline 2155 & & & Corynosoma hamanni & Lepidonotothen squamifrons & Skif Bank & Parukhin \& Lyadov (1981) \\
\hline 2156 & & & Corynosoma hamanni & Lepidonotothen squamifrons & Kerguelen Subregion & Parukhin \& Lyadov (1982) \\
\hline 2157 & & & Corynosoma hamanni & Lepidonotothen squamifrons & Crozet Islands & Parukhin \& Lyadov (1982) \\
\hline 2158 & & & Corynosoma hamanni & Lepidonotothen squamifrons & Skif Bank & Parukhin \& Lyadov (1982) \\
\hline 2159 & & & Corynosoma hamanni & Lepidonotothen squamifrons & Lena Bank & Parukhin (1986) \\
\hline 2160 & & & Corynosoma hamanni & Notothenia coriiceps & South Shetland Islands & Zdzitowiecki (1986b) \\
\hline 2161 & & & Corynosoma hamanni & Notothenia coriiceps & South Orkney Islands & Zdzitowiecki \& White (1996) \\
\hline 2162 & & & Corynosoma hamanni & Notothenia coriiceps & Admiralty Bay & Zdzitowiecki \& Laskowski \\
\hline
\end{tabular}




\begin{tabular}{|c|c|c|c|c|c|c|}
\hline Index & Phylum & Family & Species & Host & Location & References \\
\hline & & & & & & $(2004)$ \\
\hline 2163 & & & Corynosoma hamanni & Notothenia coriiceps & Vernadsky Station & $\begin{array}{l}\text { Zdzitowiecki \& Laskowski } \\
\text { (2004) }\end{array}$ \\
\hline 2164 & & & Corynosoma hamanni & Notothenia gibberifrons & South Shetland Islands & Zdzitowiecki (1986b) \\
\hline 2165 & & & Corynosoma hamanni & Notothenia gibberifrons & South Shetland Islands & Zdzitowiecki (1990c) \\
\hline 2166 & & & Corynosoma hamanni & Notothenia gibberifrons & South Georgia Island & Zdzitowiecki (1990c) \\
\hline 2167 & & & Corynosoma hamanni & Notothenia gibberifrons & South Georgia Island & Parukhin \& Lyadov (1981) \\
\hline 2168 & & & Corynosoma hamanni & Notothenia kempi & Shag Rocks & Parukhin \& Lyadov (1981) \\
\hline 2169 & & & Corynosoma hamanni & Notothenia rossii & Kerguelen Subregion & Parukhin \& Lyadov (1981) \\
\hline 2170 & & & Corynosoma hamanni & Notothenia rossii & Kherd Island & Parukhin \& Lyadov (1981) \\
\hline 2171 & & & Corynosoma hamanni & Notothenia rossii & Crozet Islands & Parukhin \& Lyadov (1981) \\
\hline 2172 & & & Corynosoma hamanni & Notothenia rossii & Ob Bank & Parukhin \& Lyadov (1981) \\
\hline 2173 & & & Corynosoma hamanni & Notothenia rossii & Georgia & Parukhin \& Lyadov (1981) \\
\hline 2174 & & & Corynosoma hamanni & Notothenia rossii & Kerguelen Subregion & Parukhin \& Lyadov (1982) \\
\hline 2175 & & & Corynosoma hamanni & Notothenia rossii & Kherd Island & Parukhin \& Lyadov (1982) \\
\hline 2176 & \multirow{13}{*}{ ACANTHOCEPHALA } & Polymorphoidea & Corynosoma hamanni & Notothenia rossii & Crozet Islands & Parukhin \& Lyadov (1982) \\
\hline 2177 & & & Corynosoma hamanni & Notothenia rossii & Skif Bank & Parukhin \& Lyadov (1982) \\
\hline 2178 & & & Corynosoma hamanni & Notothenia rossii & Ob Bank & Parukhin \& Lyadov (1982) \\
\hline 2179 & & & Corynosoma hamanni & Notothenia rossii & Ob Bank & Parukhin (1986) \\
\hline 2180 & & & Corynosoma hamanni & Notothenia rossii & Lena Bank & Parukhin (1986) \\
\hline 2181 & & & Corynosoma hamanni & Notothenia rossii & South Shetland Islands & Zdzitowiecki (1986b) \\
\hline 2182 & & & Corynosoma hamanni & Notothenia rossii & South Georgia Island & Zdzitowiecki (1990c) \\
\hline 2183 & & & Corynosoma hamanni & Nototheniidae & Kerguelen Subregion & Parukhin \& Lyadov (1982) \\
\hline 2184 & & & Corynosoma hamanni & Nototheniops larseni & Lena Bank & Parukhin (1986) \\
\hline 2185 & & & Corynosoma hamanni & Nototheniops larseni & South Shetland Islands & Palm et al. (2007) \\
\hline 2186 & & & Corynosoma hamanni & Parachaenichthys charcoti & South Shetland Islands & Zdzitowiecki (1986b) \\
\hline 2187 & & & Corynosoma hamanni & blank & blank & Zdzitowiecki (1990c) \\
\hline 2188 & & & Corynosoma hamanni & Parachaenichthys charcoti & Admiralty Bay & Zdzitowiecki (2001a) \\
\hline
\end{tabular}




\begin{tabular}{|c|c|c|c|c|c|c|}
\hline Index & Phylum & Family & Species & Host & Location & References \\
\hline 2189 & & & Corynosoma hamanni & Parachaenichthys charcoti & $\begin{array}{l}\text { Off the South Shetland } \\
\text { Islands }\end{array}$ & Zdzitowiecki (2001a) \\
\hline 2190 & & & Corynosoma hamanni & Parachaenichthys charcoti & $\begin{array}{c}\text { Admiralty Bay Bransfield } \\
\text { Strait }\end{array}$ & Zdzitowiecki (2001a) \\
\hline 2191 & & & Corynosoma hamanni & Parachaenichthys georgianus & Grytviken (South Georgia) & Zdzitowiecki (1987) \\
\hline 2192 & & & Corynosoma hamanni & Pseudochaenichthys georginanus & South Shetland Islands & Zdzitowiecki (1990c) \\
\hline 2193 & & & Corynosoma hamanni & Pseudochaenichthys georginanus & South Georgia Island & Zdzitowiecki (1990c) \\
\hline 2194 & & & Corynosoma hamanni & Trematomus bernacchii & South Shetland Islands & Zdzitowiecki (1986b) \\
\hline 2195 & & & Corynosoma hamanni & Trematomus bernacchii & Mcmurdo Sound & Moser \& Cowen (1991) \\
\hline 2196 & & & Corynosoma hamanni & Trematomus bernacchii & $\begin{array}{l}\text { Port Foster (Deception } \\
\text { Island) }\end{array}$ & Ruhl et al. (2003) \\
\hline 2197 & & & Corynosoma hamanni & Trematomus hansoni & South Shetland Islands & Zdzitowiecki (1986b) \\
\hline 2198 & & & Corynosoma hamanni & Trematomus hansoni & Grytviken (South Georgia) & Zdzitowiecki (1987) \\
\hline 2199 & & & Corynosoma hamanni & Trematomus newnesi & South Shetland Islands & Zdzitowiecki (1986b) \\
\hline 2200 & & & Corynosoma hamanni & HOST NAME NOT MENTIONED & Patagonian Shelf & Lyadov (1985) \\
\hline 2201 & & & Corynosoma hamanni & HOST NAME NOT MENTIONED & Glacial Subregion & Lyadov (1985) \\
\hline 2202 & & & Corynosoma hamanni & HOST NAME NOT MENTIONED & Kerguelen Subregion & Lyadov (1985) \\
\hline 2203 & & & Corynosoma hamanni & HOST NAME NOT MENTIONED & South Australia & Lyadov (1985) \\
\hline 2204 & & & Corynosoma pseudohamanni & blank & blank & Zdzitowiecki (1984) \\
\hline 2205 & \multirow{10}{*}{ ACANTHOCEPHALA } & Polymorphoidea & Corynosoma pseudohamanni & Artedidraco loennbergi & Weddell Sea & Zdzitowiecki (1996) \\
\hline 2206 & & & Corynosoma pseudohamanni & Chaenocephalus aceratus & South Shetland Islands & Zdzitowiecki (1986b) \\
\hline 2207 & & & Corynosoma pseudohamanni & blank & blank & Zdzitowiecki (1990c) \\
\hline 2208 & & & Corynosoma pseudohamanni & Champsocephalus gunnari & South Shetland Islands & Zdzitowiecki (1986b) \\
\hline 2209 & & & Corynosoma pseudohamanni & Chionodraco hamatus & Terra Nova Bay & Zdzitowiecki et al. (1999) \\
\hline 2210 & & & Corynosoma pseudohamanni & Chiondraco rastrospinosus & South Shetland Islands & Zdzitowiecki (1986b) \\
\hline 2211 & & & Corynosoma pseudohamanni & blank & blank & Zdzitowiecki (1990c) \\
\hline 2212 & & & Corynosoma pseudohamanni & Cryodraco antarcticus & South Shetland Islands & Zdzitowiecki (1986b) \\
\hline 2213 & & & Corynosoma pseudohamanni & blank & blank & Zdzitowiecki (1990c) \\
\hline 2214 & & & Corynosoma pseudohamanni & Cryodraco antarcticus & Terra Nova Bay & Zdzitowiecki et al. (1999) \\
\hline
\end{tabular}




\begin{tabular}{|c|c|c|c|c|c|c|}
\hline Index & Phylum & Family & Species & Host & Location & References \\
\hline 2215 & & & Corynosoma pseudohamanni & Dissostichus eleginoides & South Shetland Islands & Zdzitowiecki (1986b) \\
\hline 2216 & & & Corynosoma pseudohamanni & blank & blank & Zdzitowiecki (1990c) \\
\hline 2217 & & & Corynosoma pseudohamanni & Dissostichus eleginoides & South Georgia Island & Gaevskaya et al. (1990) \\
\hline 2218 & & & Corynosoma pseudohamanni & Dissostichus mawsoni & South Shetland Islands & Zdzitowiecki (1990c) \\
\hline 2219 & & & Corynosoma pseudohamanni & Gymnodraco acuticeps & Ross Sea & Laskowski et.al. (2005) \\
\hline 2220 & & & Corynosoma pseudohamanni & Harpagifer antarcticus & Vernadsky Station & $\begin{array}{l}\text { Laskowski \& Zdzitowiecki } \\
\text { (2005) }\end{array}$ \\
\hline 2221 & & & Corynosoma pseudohamanni & Harpagifer bispinis & South Shetland Islands & Zdzitowiecki (1986b) \\
\hline 2222 & & & Corynosoma pseudohamanni & Harpagifer georgianus & Admiralty Bay & Zdzitowiecki \& Zadrozny (1999) \\
\hline 2223 & & & Corynosoma pseudohamanni & Lepidonotothen larseni & $\begin{array}{l}\text { Port Foster (Deception } \\
\text { Island) }\end{array}$ & Ruhl et al. (2003) \\
\hline 2224 & & & Corynosoma pseudohamanni & Lindbergichthys nudifrons & South Shetland Islands & Zdzitowiecki (1986b) \\
\hline 2225 & & & Corynosoma pseudohamanni & Lepidonotothen nudifrons & $\begin{array}{l}\text { Port Foster (Deception } \\
\text { Island) }\end{array}$ & Ruhl et al. (2003) \\
\hline 2226 & & & Corynosoma pseudohamanni & Lindbergichthys nudifrons & Admiralty Bay & $\begin{array}{l}\text { Laskowski \& Zdzitowiecki } \\
\text { (2005) }\end{array}$ \\
\hline 2227 & & & Corynosoma pseudohamanni & Lindbergichthys nudifrons & Vernadsky Station & $\begin{array}{l}\text { Laskowski \& Zdzitowiecki } \\
\qquad(2005)\end{array}$ \\
\hline 2228 & & & Corynosoma pseudohamanni & Notothenia coriiceps & South Shetland Islands & Zdzitowiecki (1986b) \\
\hline 2229 & & & Corynosoma pseudohamanni & Notothenia coriiceps & South Orkney Islands & Zdzitowiecki \& White (1996) \\
\hline 2230 & & & Corynosoma pseudohamanni & Notothenia coriiceps & Adelie Land & Zdzitowiecki et.al. (1998) \\
\hline 2231 & & & Corynosoma pseudohamanni & Notothenia coriiceps & Admiralty Bay & $\begin{array}{l}\text { Zdzitowiecki \& Laskowski } \\
\text { (2004) }\end{array}$ \\
\hline 2232 & \multirow{9}{*}{ ACANTHOCEPHALA } & & Corynosoma pseudohamanni & Notothenia coriiceps & Vernadsky Station & $\begin{array}{l}\text { Zdzitowiecki \& Laskowski } \\
\text { (2004) }\end{array}$ \\
\hline 2233 & & Polymorphoidea & Corynosoma pseudohamanni & Notothenia gibberifrons & South Shetland Islands & Zdzitowiecki (1986b) \\
\hline 2234 & & & Corynosoma pseudohamanni & Notothenia rossii & South Shetland Islands & Zdzitowiecki (1986b) \\
\hline 2235 & & & Corynosoma pseudohamanni & Nototheniops nybelini & South Shetland Islands & Zdzitowiecki (1990c) \\
\hline 2236 & & & Corynosoma pseudohamanni & Parachaenichthys charcoti & South Shetland Islands & Zdzitowiecki (1986b) \\
\hline 2237 & & & Corynosoma pseudohamanni & blank & blank & Zdzitowiecki (1990c) \\
\hline 2238 & & & Corynosoma pseudohamanni & Parachaenichthys charcoti & Admiralty Bay & Zdzitowiecki (2001a) \\
\hline 2239 & & & Corynosoma pseudohamanni & Parachaenichthys charcoti & $\begin{array}{l}\text { Off the South Shetland } \\
\text { Islands }\end{array}$ & Zdzitowiecki (2001a) \\
\hline 2240 & & & Corynosoma pseudohamanni & Parachaenichthys charcoti & Bransfield Strait & Zdzitowiecki (2001a) \\
\hline
\end{tabular}




\begin{tabular}{|c|c|c|c|c|c|c|}
\hline Index & Phylum & Family & Species & Host & Location & References \\
\hline 2241 & & & Corynosoma pseudohamanni & Racovitzia glacialis & Weddell Sea & Zdzitowiecki (1996) \\
\hline 2242 & & & Corynosoma pseudohamanni & Trematomus newnesi & South Shetland Islands & Zdzitowiecki (1986b) \\
\hline 2243 & & & Corynosoma pseudohamanni & Trematomus newnesi & Admiralty Bay & $\begin{array}{l}\text { Laskowski \& Zdzitowiecki } \\
\qquad(2005)\end{array}$ \\
\hline 2244 & & & Corynosoma pseudohamanni & Trematomus newnesi & Vernadsky Station & $\begin{array}{l}\text { Laskowski \& Zdzitowiecki } \\
\qquad(2005)\end{array}$ \\
\hline 2245 & & & Corynosoma pseudohamanni & Trematomus bernacchii & South Shetland Islands & Zdzitowiecki (1986b) \\
\hline 2246 & & & Corynosoma pseudohamanni & Trematomus bernacchii & Terra Nova Bay & Zdzitowiecki et al. (1999) \\
\hline 2247 & & & Corynosoma pseudohamanni & Trematomus bernacchii & $\begin{array}{l}\text { Port Foster (Deception } \\
\text { Island) }\end{array}$ & Ruhl et al. (2003) \\
\hline 2248 & & & Corynosoma pseudohamanni & Trematomus bernacchii & Vernadsky Station & $\begin{array}{c}\text { Laskowski \& Zdzitowiecki } \\
\text { (2005) }\end{array}$ \\
\hline 2249 & & & Corynosoma pseudohamanni & Trematomus bernacchii & Admiralty Bay & $\begin{array}{l}\text { Laskowski \& Zdzitowiecki } \\
\text { (2005) }\end{array}$ \\
\hline 2250 & & & Corynosoma pseudohamanni & Trematomus hansoni & South Shetland Islands & Zdzitowiecki (1986b) \\
\hline 2251 & & & Corynosoma pseudohamanni & Trematomus hansoni & Weddell Sea & Zdzitowiecki (1996) \\
\hline 2252 & & & Corynosoma pseudohamanni & Trematomus hansoni & Terra Nova Bay & Zdzitowiecki et al. (1999) \\
\hline 2253 & & & Corynosoma pseudohamanni & Trematomus pennelli & Weddell Sea & Zdzitowiecki (1996) \\
\hline 2254 & & & Corynosoma pseudohamanni & Trematomus pennelli & Terra Nova Bay & Zdzitowiecki et al. (1999) \\
\hline 2255 & & & Corynosoma pseudohamanni & Trematomus scotti & Weddell Sea & Zdzitowiecki (1996) \\
\hline 2256 & & & Corynosoma pseudohamanni & Trematomus scotti & $\begin{array}{l}\text { Port Foster (Deception } \\
\text { Island) }\end{array}$ & Ruhl et al. (2003) \\
\hline 2257 & & & Corynosoma shackletoni & blank & blank & Zdzitowiecki (1978) \\
\hline 2258 & & & Corynosoma shackletoni & Chaenocephalus aceratus & South Shetland Islands & Zdzitowiecki (1986b) \\
\hline 2259 & & & Corynosoma shackletoni & Chaenocephalus aceratus & South Georgia Island & Zdzitowiecki (1990c) \\
\hline 2260 & & & Corynosoma shackletoni & Chaenocephalus aceratus & South Shetland Islands & Palm et al. (2007) \\
\hline 2261 & & Polymorphoidea & Corynosoma shackletoni & Notothenia coriiceps & South Shetland Islands & Zdzitowiecki (1986b) \\
\hline 2262 & & & Corynosoma shackletoni & Notothenia coriiceps & South Orkney Islands & Zdzitowiecki \& White (1996) \\
\hline 2263 & & & Corynosoma shackletoni & Notothenia coriiceps & South Shetland Islands & Palm et.al. (1998) \\
\hline 2264 & ACANTHOCEPHALA & & Corynosoma shackletoni & Notothenia coriiceps & Admiralty Bay & $\begin{array}{c}\text { Zdzitowiecki \& Laskowski } \\
\text { (2004) }\end{array}$ \\
\hline 2265 & & & Corynosoma shackletoni & Notothenia coriiceps & Vernadsky Station & $\begin{array}{l}\text { Zdzitowiecki \& Laskowski } \\
\text { (2004) }\end{array}$ \\
\hline 2266 & & & Corynosoma shackletoni & Notothenia rossi & South Shetland Islands & Zdzitowiecki (1986b) \\
\hline
\end{tabular}




\begin{tabular}{|c|c|c|c|c|c|c|}
\hline Index & Phylum & Family & Species & Host & Location & References \\
\hline 2267 & & & Corynosoma shackletoni & Notothenia rossii & South Georgia Island & Zdzitowiecki (1990c) \\
\hline 2268 & & & Corynosoma shackletoni & Nototheniops larseni & South Shetland Islands & Palm et al. (2007) \\
\hline 2269 & & & Corynosoma shackletoni & Parachaenichthys charcoti & South Shetland Islands & Zdzitowiecki (1986b) \\
\hline 2270 & & & Corynosoma shackletoni & Parachaenichthys charcoti & Admiralty Bay & Zdzitowiecki (2001a) \\
\hline 2271 & & & Corynosoma shackletoni & Parachaenichthys georgianus & South Georgia Island & Zdzitowiecki (1990c) \\
\hline 2272 & & Rhadinorhynchidae & Metacanthocephalus sp. & blank & blank & Yamaguti (1959) \\
\hline 2273 & & & Metacanthocephalus sp. & Lepidonotothen larseni & $\begin{array}{l}\text { Port Foster (Deception } \\
\text { Island) }\end{array}$ & Ruhl et al. (2003) \\
\hline 2274 & & & Metacanthocephalus sp. & Lepidonotothen nudifrons & $\begin{array}{l}\text { Port Foster (Deception } \\
\text { Island) }\end{array}$ & Ruhl et al. (2003) \\
\hline 2275 & & & Metacanthocephalus sp. & Notothenia coriiceps & Adelie Land & Zdzitowiecki (2001b) \\
\hline 2276 & & & Metacanthocephalus sp. & Parachaenichthys georgianus & Grytviken (South Georgia) & Zdzitowiecki (1987) \\
\hline 2277 & & & Metacanthocephalus sp. & Trematomus bernacchii & $\begin{array}{l}\text { Port Foster (Deception } \\
\text { Island) }\end{array}$ & Ruhl et al. (2003) \\
\hline 2278 & & & $\begin{array}{c}\text { Metacanthocephalus sp. (johnstoni and /or } \\
\text { rennicki) }\end{array}$ & Metacanthocephalus campbelli & Adelie Land & Zdzitowiecki et.al. (1998) \\
\hline 2279 & & & $\begin{array}{l}\text { Metacanthocephalus sp. (johnstoni and /or } \\
\text { rennicki) }\end{array}$ & Metacanthocephalus rennicki & Adelie Land & Zdzitowiecki et.al. (1998) \\
\hline 2280 & & & $\begin{array}{c}\text { Metacanthocephalus sp. (johnstoni and /or } \\
\text { rennicki) }\end{array}$ & Notothenia coriiceps & Adelie Land & Zdzitowiecki et.al. (1998) \\
\hline 2281 & & & Metacanthocephalus $s p .=($ M.rennicki $)$ & Chionodraco hamatus & Terranova Bay (Ross Sea) & Zdzitowiecki et al. (1999) \\
\hline 2282 & & & Metacanthocephalus $s p .=($ M.rennicki $)$ & Cryodraco antarcticus & Terranova Bay (Ross Sea) & Zdzitowiecki et al. (1999) \\
\hline 2283 & & & Metacanthocephalus $s p .=($ M.rennicki $)$ & Trematomus bernacchii & Terranova Bay (Ross Sea) & Zdzitowiecki et al. (1999) \\
\hline 2284 & & & Metacanthocephalus sp. $=($ M.rennicki $)$ & Trematomus eulepidotus & Terranova Bay (Ross Sea) & Zdzitowiecki et al. (1999) \\
\hline 2285 & & & Metacanthocephalus $s p .=($ M.rennicki $)$ & Trematomus pennelli & Terranova Bay (Ross Sea) & Zdzitowiecki et al. (1999) \\
\hline 2286 & & & Metacanthocephalus spp. & blank & blank & Yamaguti (1959) \\
\hline 2287 & & & Metacanthocephalus spp. & Chaenocephalus aceratus & South Shetland Islands & Palm et al. (2007) \\
\hline 2288 & & & Metacanthocephalus spp. & Lepidonotothen squamifrons & South Shetland Islands & Palm et al. (2007) \\
\hline 2289 & \multirow{4}{*}{ ACANTHOCEPHALA } & Rhadinorhynchidae & Metacanthocephalus spp. & Lindbergichthys nudifrons & South Shetland Islands & Palm et al. (2007) \\
\hline 2290 & & & Metacanthocephalus spp. & Muraenolepis microps & South Shetland Islands & Palm et al. (2007) \\
\hline 2291 & & & Metacanthocephalus spp. & Nototheniops larseni & South Shetland Islands & Palm et al. (2007) \\
\hline 2292 & & & Metacanthocephalus spp. & Parachaenichthys charcoti & South Shetland Islands & Palm et al. (2007) \\
\hline
\end{tabular}




\begin{tabular}{|c|c|c|c|c|c|c|}
\hline Index & Phylum & Family & Species & Host & Location & References \\
\hline 2293 & & & Metacanthocephalus spp. & Trematomus eulepidotus & South Shetland Islands & Palm et al. (2007) \\
\hline 2294 & & & Metacanthocephalus campbellii & blank & blank & Leiper \& Atkinson (1914) \\
\hline 2295 & & & Metacanthocephalus campbellii & Chionodraco hamatus & Adelie Land & Zdzitowiecki et.al. (1998) \\
\hline 2296 & & & Metacanthocephalus campbellii & Chionodraco hamatus & Terranova Bay (Ross Sea) & Zdzitowiecki et al. (1999) \\
\hline 2297 & & & Metacanthocephalus campbellii & Cryodraco antarcticus & Terranova Bay (Ross Sea) & Zdzitowiecki et al. (1999) \\
\hline 2298 & & & Metacanthocephalus campbellii & Metacanthocephalus campbellii & Adelie Land & Zdzitowiecki et.al. (1998) \\
\hline 2299 & & & Metacanthocephalus campbellii & Notothenia coriiceps & Adelie Land & Zdzitowiecki et.al. (1998) \\
\hline 2300 & & & Metacanthocephalus campbellii & Notothenia coriiceps & Adelie Land & Zdzitowiecki (2001b) \\
\hline 2301 & & & Metacanthocephalus campbellii & Parachaenichthys charcoti & Admiralty Bay & Zdzitowiecki (2001a) \\
\hline 2302 & & & Metacanthocephalus campbellii & Trematomus newnesi & $\begin{array}{l}\text { Adelie Land (Eastern } \\
\text { Antarctica) }\end{array}$ & Laskowski et.al. (2007) \\
\hline 2303 & & & Metacanthocephalus campbellii & Trematomus bernacchii & Casey Station & Rohde et al. (1998) \\
\hline 2304 & & & Metacanthocephalus campbellii & Trematomus bernacchii & Terranova Bay (Ross Sea) & Zdzitowiecki et al. (1999) \\
\hline 2305 & & & Metacanthocephalus campbellii & Trematomus eulepidotus & Grytviken (South Georgia) & Zdzitowiecki (1987) \\
\hline 2306 & & & Metacanthocephalus campbellii & Trematomus hansoni & Adelie Land & Zdzitowiecki et.al. (1998) \\
\hline 2307 & & & Metacanthocephalus campbellii & Trematomus hansoni & Terranova Bay (Ross Sea) & Zdzitowiecki et al. (1999) \\
\hline 2308 & & & Metacanthocephalus campbellii & Trematomus hansoni & Adelie Land & Zdzitowiecki (2001b) \\
\hline 2309 & & & Metacanthocephalus campbellii & Trematomus lepidorhinus & Weddell Sea & Zdzitowiecki (1996) \\
\hline 2310 & & & Metacanthocephalus dalmori & blank & blank & Zdzitowiecki (1983) \\
\hline 2311 & & & Metacanthocephalus dalmori & Artedidraco mirus & South Georgia Island & Zdzitowiecki (1990c) \\
\hline 2312 & & & Metacanthocephalus dalmori & Chaenocephalus aceratus & South Shetland Islands & Zdzitowiecki (1986c) \\
\hline 2313 & & & Metacanthocephalus dalmori & blank & blank & Palm et al. (2007) \\
\hline 2314 & & & Metacanthocephalus dalmori & Champsocephalus gunnari & South Shetland Islands & Zdzitowiecki (1986c) \\
\hline 2315 & & & Metacanthocephalus dalmori & Chiondraco rastrospinosus & South Shetland Islands & Zdzitowiecki (1990c) \\
\hline 2316 & & & Metacanthocephalus dalmori & Cryodraco antarcticus & South Shetland Islands & Zdzitowiecki (1990c) \\
\hline 2317 & & & Metacanthocephalus dalmori & Dissostichus eleginoides & South Shetland Islands & Zdzitowiecki (1990c) \\
\hline 2318 & ACANTHOCEPHAL & Rhadinorhynchidae & Metacanthocephalus dalmori & Dissostichus eleginoides & South Georgia Island & Zdzitowiecki (1990c) \\
\hline 2319 & 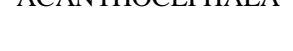 & & Metacanthocephalus dalmori & Dissostichus mawsoni & South Shetland Islands & Zdzitowiecki (1990c) \\
\hline
\end{tabular}




\begin{tabular}{|c|c|c|c|c|c|c|}
\hline Index & Phylum & Family & Species & Host & Location & References \\
\hline 2320 & & & Metacanthocephalus dalmori & Gobionotothen gibberifrons & South Shetland Islands & Palm et al. (2007) \\
\hline 2321 & & & Metacanthocephalus dalmori & Harpagifer georgianus & Admiralty Bay & Zdzitowiecki \& Zadrozny (1999) \\
\hline 2322 & & & Metacanthocephalus dalmori & Lindbergichthys nudifrons & South Shetland Islands & Zdzitowiecki (1986c) \\
\hline 2323 & & & Metacanthocephalus dalmori & blank & blank & Palm et al. (2007) \\
\hline 2324 & & & Metacanthocephalus dalmori & Lindbergichthys nudifrons & Vernadsky Station & $\begin{array}{l}\text { Laskowski \& Zdzitowiecki } \\
\qquad(2005)\end{array}$ \\
\hline 2325 & & & Metacanthocephalus dalmori & Lindbergichthys nudifrons & Admiralty Bay & $\begin{array}{l}\text { Laskowski \& Zdzitowiecki } \\
\text { (2005) }\end{array}$ \\
\hline 2326 & & & Metacanthocephalus dalmori & Muraenolepis microps & South Shetland Islands & Palm et al. (2007) \\
\hline 2327 & & & Metacanthocephalus dalmori & Notothenia coriiceps & South Shetland Islands & Zdzitowiecki (1986c) \\
\hline 2328 & & & Metacanthocephalus dalmori & Notothenia coriiceps & $\begin{array}{c}\text { Potter Cove (King George } \\
\text { Island) }\end{array}$ & Palm et.al. (1998) \\
\hline 2329 & & & Metacanthocephalus dalmori & Notothenia coriiceps & King George Island & Palm et.al. (1998) \\
\hline 2330 & & & Metacanthocephalus dalmori & Notothenia coriiceps & Admiralty Bay & $\begin{array}{l}\text { Zdzitowiecki \& Laskowski } \\
\text { (2004) }\end{array}$ \\
\hline 2331 & & & Metacanthocephalus dalmori & Notothenia coriiceps & Vernadsky Station & $\begin{array}{l}\text { Zdzitowiecki \& Laskowski } \\
\text { (2004) }\end{array}$ \\
\hline 2332 & & & Metacanthocephalus dalmori & Notothenia gibberifrons & South Shetland Islands & Zdzitowiecki (1986c) \\
\hline 2333 & & & Metacanthocephalus dalmori & Notothenia gibberifrons & South Shetland Islands & Zdzitowiecki (1990c) \\
\hline 2334 & & & Metacanthocephalus dalmori & Notothenia gibberifrons & South Georgia Island & Zdzitowiecki (1990c) \\
\hline 2335 & & & Metacanthocephalus dalmori & Notothenia rossii & South Shetland Islands & Zdzitowiecki (1986c) \\
\hline 2336 & & & Metacanthocephalus dalmori & Notothenia rossii & South Georgia Island & Zdzitowiecki (1990c) \\
\hline 2337 & & & Metacanthocephalus dalmori & Nototheniops larseni & South Shetland Islands & Palm et al. (2007) \\
\hline 2338 & & & Metacanthocephalus dalmori & Nototheniops nybelini & South Shetland Islands & Zdzitowiecki (1990c) \\
\hline 2339 & & & Metacanthocephalus dalmori & Parachaenichthys charcoti & South Shetland Islands & Zdzitowiecki (1986c) \\
\hline 2340 & & & Metacanthocephalus dalmori & Parachaenichthys charcoti & South Shetland Islands & Zdzitowiecki (1990c) \\
\hline 2341 & & & Metacanthocephalus dalmori & Parachaenichthys charcoti & Admiralty Bay & Zdzitowiecki (2001a) \\
\hline 2342 & & & Metacanthocephalus dalmori & Parachaenichthys charcoti & $\begin{array}{l}\text { Off the South Shetland } \\
\text { Islands }\end{array}$ & Zdzitowiecki (2001a) \\
\hline 2343 & & & Metacanthocephalus dalmori & Parachaenichthys charcoti & Bransfield Strait & Zdzitowiecki (2001a) \\
\hline 2344 & & & Metacanthocephalus dalmori & Parachaenichthys georgianus & South Georgia Island & Zdzitowiecki (1990c) \\
\hline 2345 & & & Metacanthocephalus dalmori & Trematomus newnesi & Vernadsky Station & $\begin{array}{l}\text { Laskowski \& Zdzitowiecki } \\
(2005)\end{array}$ \\
\hline
\end{tabular}




\begin{tabular}{|c|c|c|c|c|c|c|}
\hline Index & Phylum & Family & Species & Host & Location & References \\
\hline 2346 & \multirow{25}{*}{ ACANTHOCEPHALA } & Rhadinorhynchidae & Metacanthocephalus dalmori & Trematomus newnesi & Admiralty Bay & $\begin{array}{c}\text { Laskowski \& Zdzitowiecki } \\
\text { (2005) }\end{array}$ \\
\hline 2347 & & & Metacanthocephalus dalmori & Trematomus bernacchii & South Shetland Islands & Zdzitowiecki (1986c) \\
\hline 2348 & & & Metacanthocephalus dalmori & Trematomus bernacchii & Vernadsky Station & $\begin{array}{l}\text { Laskowski \& Zdzitowiecki } \\
\qquad(2005)\end{array}$ \\
\hline 2349 & & & Metacanthocephalus dalmori & Trematomus bernacchii & Admiralty Bay & $\begin{array}{l}\text { Laskowski \& Zdzitowiecki } \\
\text { (2005) }\end{array}$ \\
\hline 2350 & & & Metacanthocephalus dalmori & Trematomus lepidorhinus & Weddell Sea & Zdzitowiecki (1996) \\
\hline 2351 & & & Metacanthocephalus dalmori & Trematomus newnesi & South Shetland Islands & Zdzitowiecki (1986c) \\
\hline 2352 & & & Metacanthocephalus johnstoni & blank & blank & Zdzitowiecki (1983) \\
\hline 2353 & & & Metacanthocephalus johnstoni & Champsocephalus gunnari & South Shetland Islands & Zdzitowiecki (1986c) \\
\hline 2354 & & & Metacanthocephalus johnstoni & Lindbergichthys nudifrons & South Shetland Islands & Zdzitowiecki (1986c) \\
\hline 2355 & & & Metacanthocephalus johnstoni & Lindbergichthys nudifrons & Vernadsky Station & $\begin{array}{l}\text { Laskowski \& Zdzitowiecki } \\
\text { (2005) }\end{array}$ \\
\hline 2356 & & & Metacanthocephalus johnstoni & Lindbergichthys nudifrons & Admiralty Bay & $\begin{array}{l}\text { Laskowski \& Zdzitowiecki } \\
\qquad(2005)\end{array}$ \\
\hline 2357 & & & Metacanthocephalus johnstoni & Notothenia coriiceps & South Orkney Islands & Zdzitowiecki \& White (1996) \\
\hline 2358 & & & Metacanthocephalus johnstoni & Notothenia coriiceps & $\begin{array}{l}\text { Potter Cove (King George } \\
\text { Island) }\end{array}$ & Palm et.al. (1998) \\
\hline 2359 & & & Metacanthocephalus johnstoni & Notothenia coriiceps & King George Island & Palm et.al. (1998) \\
\hline 2360 & & & Metacanthocephalus johnstoni & Notothenia coriiceps & Admiralty Bay & $\begin{array}{l}\text { Zdzitowiecki \& Laskowski } \\
\text { (2004) }\end{array}$ \\
\hline 2361 & & & Metacanthocephalus johnstoni & Notothenia coriiceps & Vernadsky Station & $\begin{array}{c}\text { Zdzitowiecki \& Laskowski } \\
(2004)\end{array}$ \\
\hline 2362 & & & Metacanthocephalus johnstoni & Notothenia coriiceps & South Shetland Islands & Zdzitowiecki (1986c) \\
\hline 2363 & & & Metacanthocephalus johnstoni & Notothenia gibberifrons & South Shetland Islands & Zdzitowiecki (1986c) \\
\hline 2364 & & & Metacanthocephalus johnstoni & Notothenia rossii & South Shetland Islands & Zdzitowiecki (1986c) \\
\hline 2365 & & & Metacanthocephalus johnstoni & Notothenia rossii & South Georgia Island & Zdzitowiecki (1990c) \\
\hline 2366 & & & Metacanthocephalus johnstoni & Parachaenichthys charcoti & $\begin{array}{l}\text { Off the South Shetland } \\
\text { Islands }\end{array}$ & Zdzitowiecki (2001a) \\
\hline 2367 & & & Metacanthocephalus johnstoni & Trematomus newnesi & Vernadsky Station & $\begin{array}{c}\text { Laskowski \& Zdzitowiecki } \\
\text { (2005) }\end{array}$ \\
\hline 2368 & & & Metacanthocephalus johnstoni & Trematomus newnesi & Admiralty Bay & $\begin{array}{l}\text { Laskowski \& Zdzitowiecki } \\
\qquad(2005)\end{array}$ \\
\hline 2369 & & & Metacanthocephalus johnstoni & Trematomus newnesi & $\begin{array}{l}\text { Adelie Land (Eastern } \\
\text { Antarctica) }\end{array}$ & Laskowski et.al. (2007) \\
\hline 2370 & & & Metacanthocephalus johnstoni & Trematomus bernacchii & South Shetland Islands & Zdzitowiecki (1986c) \\
\hline
\end{tabular}




\begin{tabular}{|c|c|c|c|c|c|c|}
\hline Index & Phylum & Family & Species & Host & Location & References \\
\hline 2371 & & & Metacanthocephalus johnstoni & Trematomus bernacchii & Vernadsky Station & $\begin{array}{l}\text { Laskowski \& Zdzitowiecki } \\
\qquad(2005)\end{array}$ \\
\hline 2372 & & & Metacanthocephalus johnstoni & Trematomus bernacchii & Admiralty Bay & $\begin{array}{l}\text { Laskowski \& Zdzitowiecki } \\
\text { (2005) }\end{array}$ \\
\hline 2373 & \multirow{7}{*}{ ACANTHOCEPHALA } & Rhadinorhynchidae & Metacanthocephalus johnstoni & Trematomus eulepidotus & South Shetland Islands & Zdzitowiecki (1990c) \\
\hline 2374 & & & Metacanthocephalus johnstoni & Trematomus hansoni & South Shetland Islands & Zdzitowiecki (1986c) \\
\hline 2375 & & & Metacanthocephalus johnstoni & Trematomus hansoni & Grytviken (South Georgia) & Zdzitowiecki (1987) \\
\hline 2376 & & & Metacanthocephalus johnstoni & Trematomus newnesi & South Shetland Islands & Zdzitowiecki (1986c) \\
\hline 2377 & & & Rhadinorhynchus wheeleri & blank & blank & $\begin{array}{c}\text { Baylis }(1929)=\text { Aspersentis } \\
\text { megarhynchus }\end{array}$ \\
\hline 2378 & & & Rhadinorhynchus wheeleri & Notothenia gibberifrons & Borge Bay & Hoogesteger \& White (1981) \\
\hline 2379 & & & Rhadinorhynchus wheeleri & Notothenia gibberifrons & Signy Island & Hoogesteger \& White (1981) \\
\hline 2380 & \multirow{17}{*}{ NEMATODA } & Anisakidae & Anisakis sp. & blank & blank & Dujardin (1845) \\
\hline 2381 & & & Anisakis sp. & Chaenichthys rhinoceratus & Kerguelen Subregion & Parukhin \& Lyadov (1981) \\
\hline 2382 & & & Anisakis sp. & blank & blank & Lyadov et al. (1981) \\
\hline 2383 & & & Anisakis sp. & Chaenichthys velifer & Kerguelen Subregion & Parukhin \& Lyadov (1981) \\
\hline 2384 & & & Anisakis sp. & blank & blank & Lyadov et al. (1981) \\
\hline 2385 & & & Anisakis sp. & Chaenocephalus aceratus & Admiralty Bay & Rokicki et. al. (2009) \\
\hline 2386 & & & Anisakis sp. & Champsocephalus gunnari & Kerguelen Subregion & Lyadov et al. (1981) \\
\hline 2387 & & & Anisakis sp. & Champsocephalus gunnari & Skif Bank & Lyadov et al. (1981) \\
\hline 2388 & & & Anisakis sp. & Champsocephalus gunnari & Kerguelen Subregion & Parukhin \& Lyadov (1981) \\
\hline 2389 & & & Anisakis sp. & Champsocephalus gunnari & Skif Bank & Parukhin \& Lyadov (1981) \\
\hline 2390 & & & Anisakis sp. & Champsocephalus gunnari & Georgia & Parukhin \& Lyadov (1981) \\
\hline 2391 & & & Anisakis sp. & Chiondraco rastrospinosus & Elephant Island & Rokicki et. al. (2009) \\
\hline 2392 & & & Anisakis sp. & Chiondraco rastrospinosus & Shishkov Island & Rokicki et. al. (2009) \\
\hline 2393 & & & Anisakis sp. & Dissostichus eleginoides & Kerguelen Subregion & Parukhin \& Lyadov (1981) \\
\hline 2394 & & & Anisakis sp. & Dissostichus eleginoides & Crozet Islands & Parukhin \& Lyadov (1981) \\
\hline 2395 & & & Anisakis sp. & Dissostichus eleginoides & Ob Bank & Parukhin \& Lyadov (1981) \\
\hline 2396 & & & Anisakis sp. & Dissostichus eleginoides & Lena Bank & Parukhin \& Lyadov (1981) \\
\hline
\end{tabular}




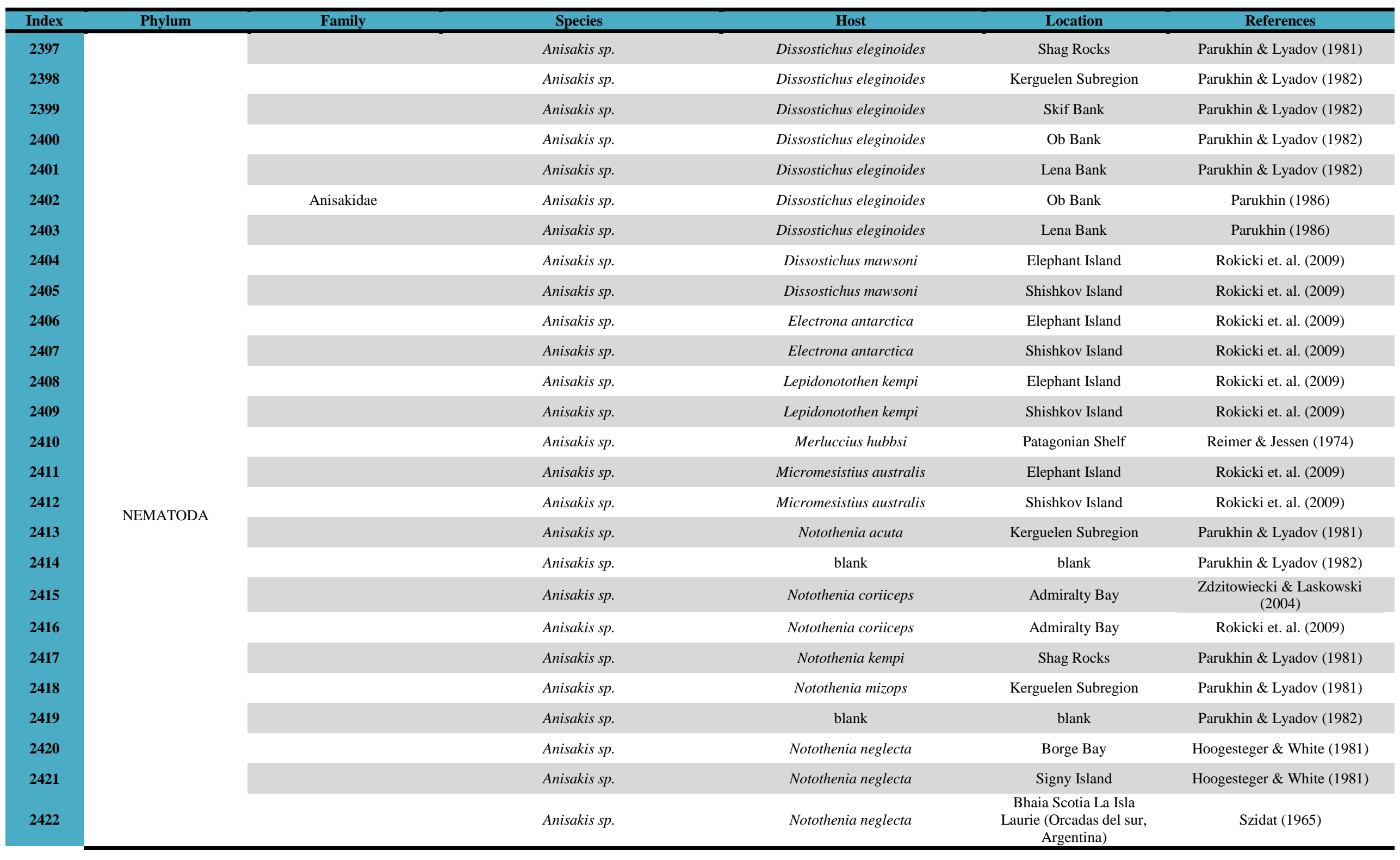




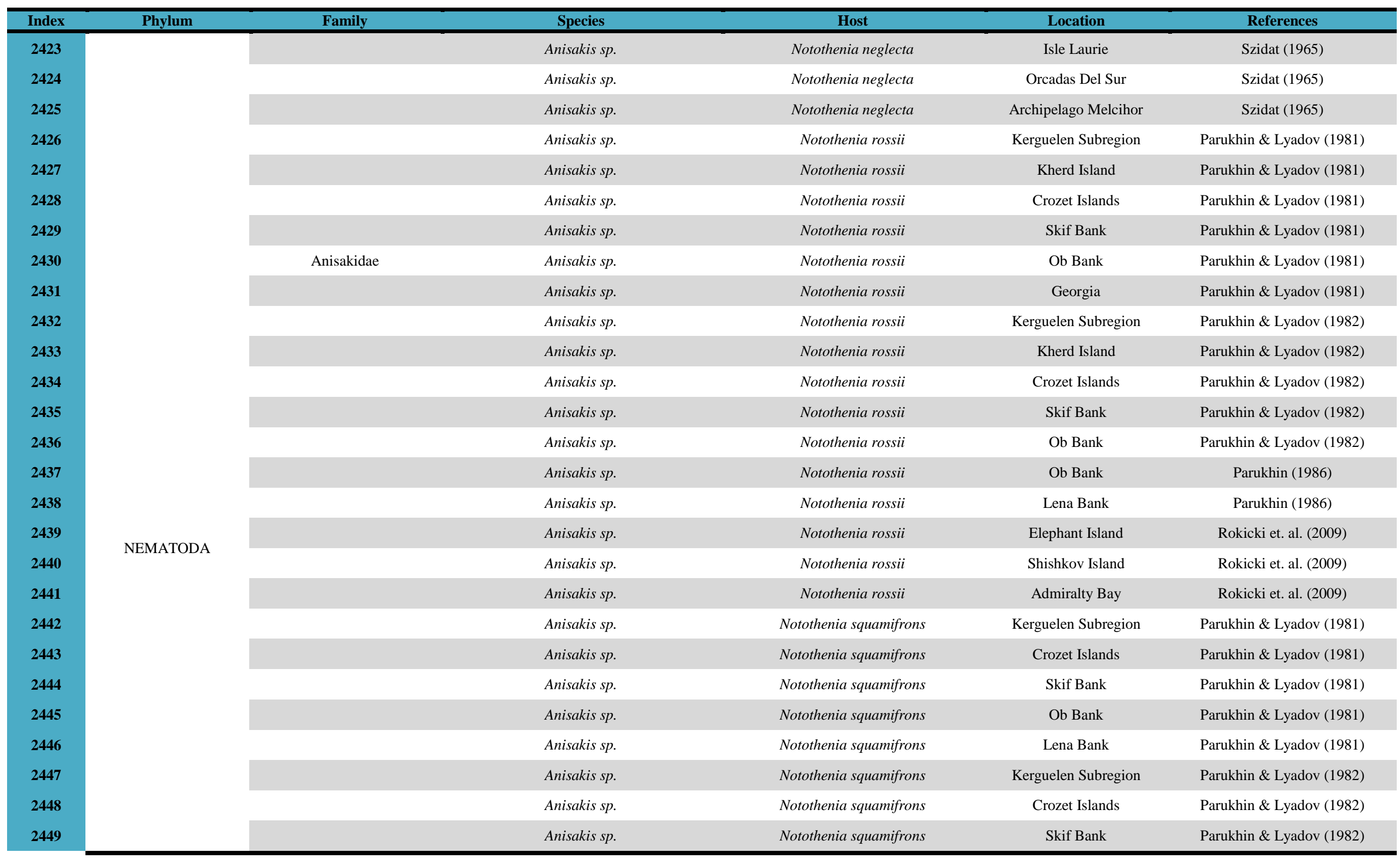




\begin{tabular}{|c|c|c|c|c|c|c|}
\hline Index & Phylum & Family & Species & Host & Location & References \\
\hline 2450 & & & Anisakis sp. & Notothenia squamifrons & Ob Bank & Parukhin \& Lyadov (1982) \\
\hline 2451 & & & Anisakis sp. & Nototheniidae & Kerguelen Subregion & Parukhin \& Lyadov (1982) \\
\hline 2452 & & & Anisakis sp. & Pseudochaenichthys georgianus & Georgia & Parukhin \& Lyadov (1981) \\
\hline 2453 & & & Anisakis sp. & Pseudochaenichthys georgianus & Elephant Island & Rokicki et. al. (2009) \\
\hline 2454 & & & Anisakis sp. & Pseudochaenichthys georgianus & Shishkov Island & Rokicki et. al. (2009) \\
\hline 2455 & & & Anisakis sp. & Trematomus eulepidotus & Elephant Island & Rokicki et. al. (2009) \\
\hline 2456 & & & Anisakis sp. & Trematomus eulepidotus & Shishkov Island & Rokicki et. al. (2009) \\
\hline 2457 & & & Anisakis sp. (Larvae) & blank & blank & Dujardin (1845) \\
\hline 2458 & & & Anisakis sp. (Larvae) & Notothenia squamifrons & Lena Bank & Parukhin (1986) \\
\hline 2459 & \multirow{18}{*}{ NEMATODA } & Anisakidae & Anisakis sp. (Larvae) & HOST NAME NOT MENTIONED & Patagonian Shelf & Lyadov (1985) \\
\hline 2460 & & & Anisakis sp. (Larvae) & HOST NAME NOT MENTIONED & Glacial Subregion & Lyadov (1985) \\
\hline 2461 & & & Anisakis sp. (Larvae) & HOST NAME NOT MENTIONED & Kerguelen Subregion & Lyadov (1985) \\
\hline 2462 & & & Anisakis sp. (Larvae) & HOST NAME NOT MENTIONED & South Australia & Lyadov (1985) \\
\hline 2463 & & & Anisakis spp. & blank & blank & Dujardin (1845) \\
\hline 2464 & & & Anisakis spp. & Dissostichus eleginoides & Shag Rocks & Brickle et al. (2005) \\
\hline 2465 & & & Anisakis spp. & Dissostichus eleginoides & South Georgia Island & Brickle et al. (2005) \\
\hline 2466 & & & Anisakis spp. & Dissostichus eleginoides & Prince Edward Island & Brickle et al. (2005) \\
\hline 2467 & & & Anisakis spp. & Dissostichus eleginoides & Heard Island & Brickle et al. (2005) \\
\hline 2468 & & & Anisakis spp. & Dissostichus eleginoides & Macquarie Island & Brickle et al. (2005) \\
\hline 2469 & & & Anisakis spp. & Dissostichus eleginoides & Ross Sea & Brickle et al. (2005) \\
\hline 2470 & & & Anisakis spp. & Dissostichus eleginoides & Falkland Islands & Brickle et al. (2006) \\
\hline 2471 & & & Anisakis pegreffii & blank & blank & $\begin{array}{c}\text { Campana-Rouget \& Biocca } \\
\text { (1955) }\end{array}$ \\
\hline 2472 & & & Anisakis pegreffii & Gymnoscopelus nicholsi & $\begin{array}{l}\text { Elephant Island (South } \\
\text { Shetland Islands) }\end{array}$ & Klimpel et al. (2010) \\
\hline 2473 & & & Anisakis simplex & blank & blank & Rudolphi (1809) \\
\hline 2474 & & & Anisakis simplex & Boreogadus saida & Arctic (Svalbard) & Dzido et.al. (2009) \\
\hline 2475 & & & Anisakis simplex & Dissostichus eleginoides & Falkland Patagonia & Gaevskaya et al. (1990) \\
\hline 2476 & & & Anisakis simplex & Dissostichus eleginoides & South Georgia Island & Gaevskaya et al. (1990) \\
\hline
\end{tabular}




\begin{tabular}{|c|c|c|c|c|c|c|}
\hline Index & Phylum & Family & Species & Host & Location & References \\
\hline 2477 & & & Anisakis simplex & Electrona carlsbergi & $\begin{array}{l}\text { Elephant Island (South } \\
\text { Shetland Islands) }\end{array}$ & Klimpel et al. (2010) \\
\hline 2478 & & & Anisakis simplex & Gymnoscopelus nicholsi & $\begin{array}{l}\text { Elephant Island (South } \\
\text { Shetland Islands) }\end{array}$ & Klimpel et al. (2010) \\
\hline 2479 & & & Anisakis simplex & Macrourus carinatus & $\begin{array}{l}\text { Falkland (Malvinas) } \\
\text { Islands }\end{array}$ & Gaevskaya \& Rodyuk (1988) \\
\hline 2480 & & & Anisakis simplex & Notothenia coriiceps & $\begin{array}{l}\text { Antarctic (King George } \\
\text { Island) }\end{array}$ & Dzido et.al. (2009) \\
\hline 2481 & & & Anisakis simplex & Notothenia rossii & $\begin{array}{l}\text { Antarctic (King George } \\
\text { Island) }\end{array}$ & Dzido et.al. (2009) \\
\hline 2482 & & & Contracaecum sp. & blank & blank & Railliet \& Henry (1912) \\
\hline 2483 & & & Contracaecum sp. & Chaenichthys rhinoceratus & Kerguelen Subregion & Parukhin \& Lyadov (1981) \\
\hline 2484 & & & Contracaecum sp. & blank & blank & Lyadov et al. (1981) \\
\hline 2485 & & & Contracaecum sp. & Chaenichthys velifer & Kerguelen Subregion & Parukhin \& Lyadov (1981) \\
\hline 2486 & \multirow{17}{*}{ NEMATODA } & & Contracaecum sp. & blank & blank & Lyadov et al. (1981) \\
\hline 2487 & & Anisakidae & Contracaecum sp. & Chaenocephalus aceratus & South Shetland Islands & Siegel (1980a) \\
\hline 2488 & & & Contracaecum sp. & Chaenocephalus aceratus & South Georgia Island & Siegel (1980a) \\
\hline 2489 & & & Contracaecum $s p$ & Chaenocephalus aceratus & South Orkney Islands & Siegel (1980a) \\
\hline 2490 & & & Contracaecum sp. & Chaenocephalus aceratus & Elephant Island & Siegel (1980a) \\
\hline 2491 & & & Contracaecum $s p$ & Chaenocephalus aceratus & South Georgia Island & Siegel (1980b) \\
\hline 2492 & & & Contracaecum sp. & Chaenocephalus aceratus & South Shetland Islands & Siegel (1980b) \\
\hline 2493 & & & Contracaecum sp. & Chaenocephalus aceratus & Georgia & Parukhin \& Lyadov (1981) \\
\hline 2494 & & & Contracaecum sp. & Champsocephalus gunnari & South Shetland Islands & Siegel (1980a) \\
\hline 2495 & & & Contracaecum sp. & Champsocephalus gunnari & South Georgia Island & Siegel (1980a) \\
\hline 2496 & & & Contracaecum sp. & Champsocephalus gunnari & South Orkney Islands & Siegel (1980a) \\
\hline 2497 & & & Contracaecum sp. & Champsocephalus gunnari & Elephant Island & Siegel (1980a) \\
\hline 2498 & & & Contracaecum sp. & Chionodraco sp. & South Shetland Islands & Siegel (1980a) \\
\hline 2499 & & & Contracaecum sp. & Chionodraco sp. & South Georgia Island & Siegel (1980a) \\
\hline 2500 & & & Contracaecum sp. & Chionodraco sp. & South Orkney Islands & Siegel (1980a) \\
\hline 2501 & & & Contracaecum sp. & Chionodraco sp. & Elephant Island & Siegel (1980a) \\
\hline 2502 & & & Contracaecum $s p$. & Chionodraco sp. & South Georgia Island & Siegel (1980b) \\
\hline
\end{tabular}




\begin{tabular}{|c|c|c|c|c|c|c|}
\hline Index & Phylum & Family & Species & Host & Location & References \\
\hline 2503 & & & Contracaecum sp. & Dissostichus eleginoides & Kerguelen Subregion & Parukhin \& Lyadov (1981) \\
\hline 2504 & & & Contracaecum sp. & Dissostichus eleginoides & Crozet Islands & Parukhin \& Lyadov (1981) \\
\hline 2505 & & & Contracaecum $s p$. & Dissostichus eleginoides & Ob Bank & Parukhin \& Lyadov (1981) \\
\hline 2506 & & & Contracaecum sp. & Dissostichus eleginoides & Lena Bank & Parukhin \& Lyadov (1981) \\
\hline 2507 & & & Contracaecum sp. & Dissostichus eleginoides & Shag Rocks & Parukhin \& Lyadov (1981) \\
\hline 2508 & & & Contracaecum sp. & Dissostichus eleginoides & Kerguelen Subregion & Parukhin \& Lyadov (1982) \\
\hline 2509 & & & Contracaecum sp. & Dissostichus eleginoides & Skif Bank & Parukhin \& Lyadov (1982) \\
\hline 2510 & & & Contracaecum sp. & Dissostichus eleginoides & Ob Bank & Parukhin \& Lyadov (1982) \\
\hline 2511 & & & Contracaecum sp. & Dissostichus eleginoides & Lena Bank & Parukhin \& Lyadov (1982) \\
\hline 2512 & & & Contracaecum sp. & Dissostichus eleginoides & South Georgia Island & Gaevskaya et al. (1990) \\
\hline 2513 & & & Contracaecum sp. & Dissostichus eleginoides & Shag Rocks & Brickle et al. (2005) \\
\hline 2514 & & & Contracaecum sp. & Dissostichus eleginoides & South Georgia Island & Brickle et al. (2005) \\
\hline 2515 & & & Contracaecum sp. & Dissostichus eleginoides & Heard Island & Brickle et al. (2005) \\
\hline 2516 & \multirow{13}{*}{ NEMATODA } & Anisakidae & Contracaecum sp. & Macrourus carinatus & $\begin{array}{c}\text { Falkland (Malvinas) } \\
\text { Islands }\end{array}$ & Gaevskaya \& Rodyuk (1988) \\
\hline 2517 & & & Contracaecum sp. & Merluccius hubbsi & Patagonian Shelf & Reimer \& Jessen (1974) \\
\hline 2518 & & & Contracaecum sp. & Notothenia acuta & Kerguelen Subregion & Parukhin \& Lyadov (1981) \\
\hline 2519 & & & Contracaecum sp. & Notothenia coriiceps & King George Island & Dzido et.al. (2009) \\
\hline 2520 & & & Contracaecum sp. & Notothenia gibberifrons & Georgia & Parukhin \& Lyadov (1981) \\
\hline 2521 & & & Contracaecum sp. & Notothenia kempi & Shag Rocks & Parukhin \& Lyadov (1981) \\
\hline 2522 & & & Contracaecum sp. & Notothenia larseni & Lena Bank & Parukhin (1986) \\
\hline 2523 & & & Contracaecum sp. & Notothenia larseni & Ob Bank & Parukhin (1986) \\
\hline 2524 & & & Contracaecum sp. & Notothenia mizops & Kerguelen Subregion & Parukhin \& Lyadov (1981) \\
\hline 2525 & & & Contracaecum sp. & Notothenia neglecta & $\begin{array}{c}\text { Bhaia Scotia La Isla } \\
\text { Laurie (Orcadas del sur, } \\
\text { Argentina) }\end{array}$ & Szidat (1965) \\
\hline 2526 & & & Contracaecum sp. & Notothenia neglecta & Isle Laurie & Szidat (1965) \\
\hline 2527 & & & Contracaecum sp. & Notothenia neglecta & Orcadas Del Sur & Szidat (1965) \\
\hline 2528 & & & Contracaecum $s p$. & Notothenia neglecta & Archipelago Melcihor & Szidat (1965) \\
\hline
\end{tabular}




\begin{tabular}{|c|c|c|c|c|c|c|}
\hline Index & Phylum & Family & Species & Host & Location & References \\
\hline 2529 & & & Contracaecum sp. & Notothenia neglecta & Borge Bay & Hoogesteger \& White (1981) \\
\hline 2530 & & & Contracaecum sp. & Notothenia neglecta & Signy Island & Hoogesteger \& White (1981) \\
\hline 2531 & & & Contracaecum sp. & Notothenia ramsayi & Folkland & Parukhin \& Lyadov (1981) \\
\hline 2532 & & & Contracaecum sp. & Notothenia rossii & Kerguelen Subregion & Parukhin \& Lyadov (1981) \\
\hline 2533 & & & Contracaecum sp. & Notothenia rossii & Kherd Island & Parukhin \& Lyadov (1981) \\
\hline 2534 & & & Contracaecum sp. & Notothenia rossii & Crozet Islands & Parukhin \& Lyadov (1981) \\
\hline 2535 & & & Contracaecum sp. & Notothenia rossii & Skif Bank & Parukhin \& Lyadov (1981) \\
\hline 2536 & & & Contracaecum sp. & Notothenia rossii & Ob Bank & Parukhin \& Lyadov (1981) \\
\hline 2537 & & & Contracaecum sp. & Notothenia rossii & Georgia & Parukhin \& Lyadov (1981) \\
\hline 2538 & & & Contracaecum sp. & Notothenia rossii & Kerguelen Subregion & Parukhin \& Lyadov (1982) \\
\hline 2539 & & & Contracaecum sp. & Notothenia rossii & Crozet Islands & Parukhin \& Lyadov (1982) \\
\hline 2540 & & & Contracaecum sp. & Notothenia rossii & Skif Bank & Parukhin \& Lyadov (1982) \\
\hline 2541 & & & Contracaecum sp. & Notothenia rossii & Ob Bank & Parukhin \& Lyadov (1982) \\
\hline 2542 & & & Contracaecum sp. & Notothenia rossii rossii & Ob Bank & Parukhin (1986) \\
\hline 2543 & \multirow{13}{*}{ NEMATODA } & & Contracaecum sp. & Notothenia squamifrons & Kerguelen Subregion & Parukhin \& Lyadov (1981) \\
\hline 2544 & & Anisakidae & Contracaecum sp. & Notothenia squamifrons & Crozet Islands & Parukhin \& Lyadov (1981) \\
\hline 2545 & & & Contracaecum $s p$. & Notothenia squamifrons & Skif Bank & Parukhin \& Lyadov (1981) \\
\hline 2546 & & & Contracaecum sp. & Notothenia squamifrons & Ob Bank & Parukhin \& Lyadov (1981) \\
\hline 2547 & & & Contracaecum sp. & Notothenia squamifrons & Lena Bank & Parukhin \& Lyadov (1981) \\
\hline 2548 & & & Contracaecum sp. & Notothenia squamifrons & Kerguelen Subregion & Parukhin \& Lyadov (1982) \\
\hline 2549 & & & Contracaecum sp. & Notothenia squamifrons & Kherd Island & Parukhin \& Lyadov (1982) \\
\hline 2550 & & & Contracaecum sp. & Notothenia squamifrons & Crozet Islands & Parukhin \& Lyadov (1982) \\
\hline 2551 & & & Contracaecum sp. & Notothenia squamifrons & Skif Bank & Parukhin \& Lyadov (1982) \\
\hline 2552 & & & Contracaecum sp. & Notothenia squamifrons & Ob Bank & Parukhin \& Lyadov (1982) \\
\hline 2553 & & & Contracaecum sp. & Notothenia squamifrons & Lena Bank & Parukhin \& Lyadov (1982) \\
\hline 2554 & & & Contracaecum sp. & Notothenia squamifrons & Lena Bank & Parukhin (1986) \\
\hline 2555 & & & Contracaecum sp. & Nototheniidae & Kerguelen Subregion & Parukhin \& Lyadov (1982) \\
\hline
\end{tabular}




\begin{tabular}{|c|c|c|c|c|c|c|}
\hline Index & Phylum & Family & Species & Host & Location & References \\
\hline 2556 & & & Contracaecum sp. & Parachaenichthys charcoti & South Shetland Islands & Szidat \& Graefe (1967) \\
\hline 2557 & & & Contracaecum sp. & Prionodraco evansii & Weddell Sea & Kock et al. (1984) \\
\hline 2558 & & & Contracaecum sp. & Pseudochaenichthys georgianus & South Georgia Island & Siegel (1980b) \\
\hline 2559 & & & Contracaecum sp. & Pseudochaenichthys georgianus & South Orkney Islands & Siegel (1980b) \\
\hline 2560 & & & Contracaecum sp. & Pseudochaenichthys georgianus & South Shetland Islands & Siegel (1980b) \\
\hline 2561 & & & Contracaecum sp. & Pseudocheanichthys georgianus & South Shetland Islands & Siegel (1980a) \\
\hline 2562 & & & Contracaecum sp. & Pseudocheanichthys georgianus & South Georgia Island & Siegel (1980a) \\
\hline 2563 & & & Contracaecum sp. & Pseudocheanichthys georgianus & South Orkney Islands & Siegel (1980a) \\
\hline 2564 & & & Contracaecum sp. & Pseudocheanichthys georgianus & Elephant Island & Siegel (1980a) \\
\hline 2565 & & & Contracaecum sp. & Trematomus bernacchii & Mcmurdo Sound & Moser \& Cowen (1991) \\
\hline 2566 & & & Contracaecum sp. & Trematomus scotti & Weddell Sea & Kock et al. (1984) \\
\hline 2567 & & & Contracaecum sp. & HOST NAME NOT MENTIONED & Patagonian Shelf & Lyadov (1985) \\
\hline 2568 & & & Contracaecum sp. & HOST NAME NOT MENTIONED & Glacial Subregion & Lyadov (1985) \\
\hline 2569 & & & Contracaecum sp. & HOST NAME NOT MENTIONED & Kerguelen Subregion & Lyadov (1985) \\
\hline 2570 & & & Contracaecum sp. & HOST NAME NOT MENTIONED & South Australia & Lyadov (1985) \\
\hline 2571 & & & Contracaecum spp. & blank & blank & Railliet \& Henry (1912) \\
\hline 2572 & & & Contracaecum spp. & Acanthodraco dewitti & King George Island & Rokicki et. al. (2009) \\
\hline 2573 & & Anisakidae & Contracaecum spp. & Acanthodraco dewitti & Admiralty Bay & Rokicki et. al. (2009) \\
\hline 2574 & & & Contracaecum spp. & Chaenocephalus aceratus & $\begin{array}{c}\text { Elephant \& Shishkov } \\
\text { Islands }\end{array}$ & Rokicki et. al. (2009) \\
\hline 2575 & & & Contracaecum spp. & Chaenocephalus aceratus & King George Island & Rokicki et. al. (2009) \\
\hline 2576 & & & Contracaecum spp. & Chaenocephalus aceratus & Admiralty Bay & Rokicki et. al. (2009) \\
\hline 2577 & NEMATODA & & Contracaecum spp. & Chaenodraco wilsoni & $\begin{array}{l}\text { Elephant \& Shishkov } \\
\text { Islands }\end{array}$ & Rokicki et. al. (2009) \\
\hline 2578 & & & Contracaecum spp. & Champsocephalus gunnari & $\begin{array}{c}\text { Elephant \& Shishkov } \\
\text { Islands }\end{array}$ & Rokicki et. al. (2009) \\
\hline 2579 & & & Contracaecum spp. & Champsocephalus gunnari & King George Island & Rokicki et. al. (2009) \\
\hline 2580 & & & Contracaecum spp. & Champsocephalus gunnari & Admiralty Bay & Rokicki et. al. (2009) \\
\hline 2581 & & & Contracaecum spp. & Chiondraco rastrospinosus & $\begin{array}{l}\text { Elephant \& Shishkov } \\
\text { Islands }\end{array}$ & Rokicki et. al. (2009) \\
\hline
\end{tabular}




\begin{tabular}{|c|c|c|c|c|c|c|}
\hline Index & Phylum & Family & Species & Host & Location & References \\
\hline 2582 & & & Contracaecum spp. & Chiondraco rastrospinosus & King George Island & Rokicki et. al. (2009) \\
\hline 2583 & & & Contracaecum spp. & Chiondraco rastrospinosus & Admiralty Bay & Rokicki et. al. (2009) \\
\hline 2584 & & & Contracaecum spp. & Chionodraco hamatus & Adelie Land & Zdzitowiecki et.al. (1998) \\
\hline 2585 & & & Contracaecum spp. & blank & blank & Zdzitowiecki (2001b) \\
\hline 2586 & & & Contracaecum spp. & Cryodraco antarcticus & $\begin{array}{l}\text { Elephant \& Shishkov } \\
\text { Islands }\end{array}$ & Rokicki et. al. (2009) \\
\hline 2587 & & & Contracaecum spp. & Cryodraco antarcticus & King George Island & Rokicki et. al. (2009) \\
\hline 2588 & & & Contracaecum spp. & Cryodraco antarcticus & Admiralty Bay & Rokicki et. al. (2009) \\
\hline 2589 & & & Contracaecum spp. & Cygnodraco mawsoni & Ross Sea & Laskowski et.al. (2005) \\
\hline 2590 & & & Contracaecum spp. & Dissostichus eleginoides & Falkland Islands & Brickle et al. (2006) \\
\hline 2591 & & & Contracaecum spp. & Gobionotothen gibberifrons & $\begin{array}{l}\text { Elephant \& Shishkov } \\
\text { Islands }\end{array}$ & Rokicki et. al. (2009) \\
\hline 2592 & & & Contracaecum spp. & Gobionotothen gibberifrons & King George Island & Rokicki et. al. (2009) \\
\hline 2593 & & & Contracaecum spp. & Gobionotothen gibberifrons & Admiralty Bay & Rokicki et. al. (2009) \\
\hline 2594 & & & Contracaecum spp. & Gymnodraco acuticeps & Ross Sea & Laskowski et.al. (2005) \\
\hline 2595 & & & Contracaecum spp. & Harpagifer antarcticus & Vernadsky Station & $\begin{array}{l}\text { Laskowski \& Zdzitowiecki } \\
\qquad(2005)\end{array}$ \\
\hline 2596 & & & Contracaecum spp. & Harpagifer georgianus & Admiralty Bay & Zdzitowiecki \& Zadrozny (1999) \\
\hline 2597 & & & Contracaecum spp. & Harpagifer georgianus & Off Elephant Island & Zdzitowiecki \& Zadrozny (1999) \\
\hline 2598 & & & Contracaecum spp. & Lepidonotothen kempi & $\begin{array}{l}\text { Elephant \& Shishkov } \\
\text { Islands }\end{array}$ & Rokicki et. al. (2009) \\
\hline 2599 & & & Contracaecum spp. & Lepidonotothen nudifrons & $\begin{array}{l}\text { Port Foster (Deception } \\
\text { Island) }\end{array}$ & Ruhl et al. (2003) \\
\hline 2600 & & & Contracaecum spp. & Lindbergichthys nudifrons & Vernadsky Station & $\begin{array}{c}\text { Laskowski \& Zdzitowiecki } \\
\text { (2005) }\end{array}$ \\
\hline 2601 & & Anisakidae & Contracaecum spp. & Lindbergichthys nudifrons & Admiralty Bay & $\begin{array}{l}\text { Laskowski \& Zdzitowiecki } \\
\text { (2005) }\end{array}$ \\
\hline 2602 & & & Contracaecum spp. & Lepidonotothen nudifrons & King George Island & Rokicki et. al. (2009) \\
\hline 2603 & & & Contracaecum spp. & Lepidonotothen nudifrons & Admiralty Bay & Rokicki et. al. (2009) \\
\hline 2604 & NEMATODA & & Contracaecum spp. & Lycenchelus aratrirostris & $\begin{array}{l}\text { Elephant \& Shishkov } \\
\text { Islands }\end{array}$ & Rokicki et. al. (2009) \\
\hline 2605 & & & Contracaecum spp. & Micromesistius australis & $\begin{array}{l}\text { Elephant \& Shishkov } \\
\text { Islands }\end{array}$ & Rokicki et. al. (2009) \\
\hline 2606 & & & Contracaecum spp. & Notothenia coriiceps & Adelie Land & Zdzitowiecki et.al. (1998) \\
\hline 2607 & & & Contracaecum spp. & Notothenia coriiceps & Adelie Land & Zdzitowiecki (2001b) \\
\hline
\end{tabular}




\begin{tabular}{|c|c|c|c|c|c|c|}
\hline Index & Phylum & Family & Species & Host & Location & References \\
\hline 2608 & & & Contracaecum spp. & Notothenia coriiceps & Admiralty Bay & $\begin{array}{c}\text { Zdzitowiecki \& Laskowski } \\
\text { (2004) }\end{array}$ \\
\hline 2609 & & & Contracaecum spp. & Notothenia coriiceps & Vernadsky Station & $\begin{array}{c}\text { Zdzitowiecki \& Laskowski } \\
\text { (2004) }\end{array}$ \\
\hline 2610 & & & Contracaecum spp. & Notothenia coriiceps & King George Island & Rokicki et. al. (2009) \\
\hline 2611 & & & Contracaecum spp. & Notothenia coriiceps & Admiralty Bay & Rokicki et. al. (2009) \\
\hline 2612 & & & Contracaecum spp. & Notothenia rossii & King George Island & Rokicki et. al. (2009) \\
\hline 2613 & & & Contracaecum spp. & Notothenia rossii & Admiralty Bay & Rokicki et. al. (2009) \\
\hline 2614 & & & Contracaecum spp. & Notothenia rossii & $\begin{array}{l}\text { Elephant \& Shishkov } \\
\text { Islands }\end{array}$ & Rokicki et. al. (2009) \\
\hline 2615 & & & Contracaecum spp. & Nototheniops nybelini & $\begin{array}{l}\text { Elephant \& Shishkov } \\
\text { Islands }\end{array}$ & Rokicki et. al. (2009) \\
\hline 2616 & & & Contracaecum spp. & Nototheniops nybelini & King George Island & Rokicki et. al. (2009) \\
\hline 2617 & & & Contracaecum spp. & Nototheniops nybelini & Admiralty Bay & Rokicki et. al. (2009) \\
\hline 2618 & & & Contracaecum spp. & Pagetopsis macropterus & $\begin{array}{l}\text { Elephant \& Shishkov } \\
\text { Islands }\end{array}$ & Rokicki et. al. (2009) \\
\hline 2619 & & & Contracaecum spp. & Parachaenichthys charcoti & Admiralty Bay & Zdzitowiecki (2001a) \\
\hline 2620 & & & Contracaecum spp. & Parachaenichthys charcoti & $\begin{array}{l}\text { Off the South Shetland } \\
\text { Islands }\end{array}$ & Zdzitowiecki (2001a) \\
\hline 2621 & & & Contracaecum spp. & Parachaenichthys charcoti & Bransfield Strait & Zdzitowiecki (2001a) \\
\hline 2622 & & & Contracaecum spp. & Pleurogramma antarcticum & $\begin{array}{c}\text { Elephant \& Shishkov } \\
\text { Islands }\end{array}$ & Rokicki et. al. (2009) \\
\hline 2623 & & & Contracaecum spp. & Pleurogramma antarcticum & King George Island & Rokicki et. al. (2009) \\
\hline 2624 & & & Contracaecum spp. & Pleurogramma antarcticum & Admiralty Bay & Rokicki et. al. (2009) \\
\hline 2625 & & & Contracaecum spp. & Trematomus bernacchii & $\begin{array}{l}\text { Port Foster (Deception } \\
\text { Island) }\end{array}$ & Ruhl et al. (2003) \\
\hline 2626 & & & Contracaecum spp. & Trematomus bernacchii & Vernadsky Station & $\begin{array}{c}\text { Laskowski \& Zdzitowiecki } \\
\text { (2005) }\end{array}$ \\
\hline 2627 & & & Contracaecum spp. & Trematomus bernacchii & Admiralty Bay & $\begin{array}{l}\text { Laskowski \& Zdzitowiecki } \\
\qquad(2005)\end{array}$ \\
\hline 2628 & \multirow{5}{*}{ NEMATODA } & Anisakidae & Contracaecum spp. & Trematomus bernacchii & King George Island & Rokicki et. al. (2009) \\
\hline 2629 & & & Contracaecum spp. & Trematomus bernacchii & Admiralty Bay & Rokicki et. al. (2009) \\
\hline 2630 & & & Contracaecum spp. & Trematomus eulepidotus & $\begin{array}{c}\text { Elephant \& Shishkov } \\
\text { Islands }\end{array}$ & Rokicki et. al. (2009) \\
\hline 2631 & & & Contracaecum spp. & Trematomus hansoni & Adelie Land & Zdzitowiecki et.al. (1998) \\
\hline 2632 & & & Contracaecum spp. & Trematomus hansoni & King George Island & Rokicki et. al. (2009) \\
\hline
\end{tabular}




\begin{tabular}{|c|c|c|c|c|c|c|}
\hline Index & Phylum & Family & Species & Host & Location & References \\
\hline 2633 & & & Contracaecum spp. & Trematomus hansoni & Admiralty Bay & Rokicki et. al. (2009) \\
\hline 2634 & & & Contracaecum spp. & Trematomus newnesi & Vernadsky Station & $\begin{array}{c}\text { Laskowski \& Zdzitowiecki } \\
\text { (2005) }\end{array}$ \\
\hline 2635 & & & Contracaecum spp. & Trematomus newnesi & Admiralty Bay & $\begin{array}{l}\text { Laskowski \& Zdzitowiecki } \\
\text { (2005) }\end{array}$ \\
\hline 2636 & & & Contracaecum spp. & Trematomus newnesi & King George Island & Rokicki et. al. (2009) \\
\hline 2637 & & & Contracaecum spp. & Trematomus newnesi & Admiralty Bay & Rokicki et. al. (2009) \\
\hline 2638 & & & Contracaecum spp. & Trematomus scottii & $\begin{array}{l}\text { Port Foster (Deception } \\
\text { Island) }\end{array}$ & Ruhl et al. (2003) \\
\hline 2639 & & & Contracaecum nototheniae & blank & blank & Johnston \& Mawson (1945) \\
\hline 2640 & & & Contracaecum nototheniae & Dissostichus eleginoides & Kerguelen Subregion & Parukhin \& Lyadov (1981) \\
\hline 2641 & & & Contracaecum nototheniae & Dissostichus eleginoides & Crozet Islands & Parukhin \& Lyadov (1981) \\
\hline 2642 & & & Contracaecum nototheniae & Dissostichus eleginoides & Ob Bank & Parukhin \& Lyadov (1981) \\
\hline 2643 & & & Contracaecum nototheniae & Dissostichus eleginoides & Lena Bank & Parukhin \& Lyadov (1981) \\
\hline 2644 & & & Contracaecum nototheniae & Dissostichus eleginoides & Shag Rocks & Parukhin \& Lyadov (1981) \\
\hline 2645 & & & Contracaecum nototheniae & Dissostichus eleginoides & Kerguelen Subregion & Parukhin \& Lyadov (1982) \\
\hline 2646 & & & Contracaecum nototheniae & Dissostichus eleginoides & Skif Bank & Parukhin \& Lyadov (1982) \\
\hline 2647 & & & Contracaecum nototheniae & Dissostichus eleginoides & Ob Bank & Parukhin \& Lyadov (1982) \\
\hline 2648 & & & Contracaecum nototheniae & Dissostichus eleginoides & Lena Bank & Parukhin \& Lyadov (1982) \\
\hline 2649 & & & Contracaecum nototheniae & Dissostichus eleginoides & Ob Bank & Parukhin (1986) \\
\hline 2650 & & & Contracaecum nototheniae & Dissostichus eleginoides & Lena Bank & Parukhin (1986) \\
\hline 2651 & & & Contracaecum nototheniae & Notothenia rossii & Kerguelen Subregion & Parukhin \& Lyadov (1982) \\
\hline 2652 & & & Contracaecum nototheniae & Notothenia rossii & Kherd Island & Parukhin \& Lyadov (1982) \\
\hline 2653 & & & Contracaecum nototheniae & Notothenia rossii & Crozet Islands & Parukhin \& Lyadov (1982) \\
\hline 2654 & & & Contracaecum nototheniae & Notothenia rossii & Skif Bank & Parukhin \& Lyadov (1982) \\
\hline 2655 & & & Contracaecum nototheniae & Notothenia rossii & Ob Bank & Parukhin \& Lyadov (1982) \\
\hline 2656 & & & Contracaecum nototheniae & Notothenia rossii & Kerguelen Subregion & Parukhin \& Lyadov (1981) \\
\hline 2657 & \multirow{2}{*}{ NEMATODA } & Anisakidae & Contracaecum nototheniae & Notothenia rossii & Kherd Island & Parukhin \& Lyadov (1981) \\
\hline 2658 & & & Contracaecum nototheniae & Notothenia rossii & Crozet Islands & Parukhin \& Lyadov (1981) \\
\hline
\end{tabular}




\begin{tabular}{|c|c|c|c|c|c|c|}
\hline Index & Phylum & Family & Species & Host & Location & References \\
\hline 2659 & & & Contracaecum nototheniae & Notothenia rossii & Skif Bank & Parukhin \& Lyadov (1981) \\
\hline 2660 & & & Contracaecum nototheniae & Notothenia rossii & Ob Bank & Parukhin \& Lyadov (1981) \\
\hline 2661 & & & Contracaecum nototheniae & Notothenia rossii & Ob Bank & Parukhin (1986) \\
\hline 2662 & & & Contracaecum nototheniae & Notothenia rossii & Lena Bank & Parukhin (1986) \\
\hline 2663 & & & Contracaecum nototheniae & Nototheniidae & Kerguelen Subregion & Parukhin \& Lyadov (1982) \\
\hline 2664 & & & Contracaecum nototheniae & HOST NAME NOT MENTIONED & Glacial Subregion & Lyadov (1985) \\
\hline 2665 & & & Contracaecum nototheniae & HOST NAME NOT MENTIONED & Kerguelen Subregion & Lyadov (1985) \\
\hline 2666 & & & Contracaecum osculatum & blank & blank & Rudolphi (1802) \\
\hline 2667 & & & Contracaecum osculatum & Chaenocephalus aceratus & South Shetland Islands & Palm et al. (2007) \\
\hline 2668 & & & Contracaecum osculatum & Chionodraco myersi & Weddell Sea & Klöser et.al. (1992) \\
\hline 2669 & & & Contracaecum osculatum & Cryodraco antarcticus & Weddell Sea & Klöser et.al. (1992) \\
\hline 2670 & & & Contracaecum osculatum & Dissostichus eleginoides & Falkland-Patagonia & Gaevskaya et al. (1990) \\
\hline 2671 & & & Contracaecum osculatum & Gerlachea australis & Weddell Sea & Klöser et.al. (1992) \\
\hline 2672 & & & Contracaecum osculatum & Gobionotothen gibberifrons & South Shetland Islands & Palm et al. (2007) \\
\hline 2673 & & & Contracaecum osculatum & Lepidonotothen larseni & South Shetland Islands & Palm et al. (2007) \\
\hline 2674 & & & Contracaecum osculatum & Lepidonotothen nudifrons & South Shetland Islands & Palm et al. (2007) \\
\hline 2675 & & & Contracaecum osculatum & Lepidonotothen squamifrons & South Shetland Islands & Palm et al. (2007) \\
\hline 2676 & & & Contracaecum osculatum & Muraenolepis microps & South Shetland Islands & Palm et al. (2007) \\
\hline 2677 & & & Contracaecum osculatum & Notothenia coriiceps & $\begin{array}{l}\text { Potter Cove (King George } \\
\text { Island) }\end{array}$ & Palm et.al. (1998) \\
\hline 2678 & & & Contracaecum osculatum & Notothenia coriiceps & King George Island & Palm et.al. (1998) \\
\hline 2679 & & & Contracaecum osculatum & Parachaenichthys charcoti & South Shetland Islands & Palm et al. (2007) \\
\hline 2680 & & & Contracaecum osculatum & Pleurogramma antarcticus & Weddell Sea & Klöser et.al. (1992) \\
\hline 2681 & & & Contracaecum osculatum & Trematomus newnesi & $\begin{array}{l}\text { Adelie Land (Eastern } \\
\text { Antarctica) }\end{array}$ & Laskowski et.al. (2007) \\
\hline 2682 & & & Contracaecum osculatum & Trematomus eulepidotus & South Shetland Islands & Palm et al. (2007) \\
\hline 2683 & & & Contracaecum osculatum & Trematomus scotti & Weddell Sea & Klöser et.al. (1992) \\
\hline 2684 & & & Contracaecum radiatum & blank & blank & Linstow (1907) \\
\hline 2685 & & & Contracaecum radiatum & Chaenocephalus aceratus & South Shetland Islands & Palm et al. (2007) \\
\hline
\end{tabular}




\begin{tabular}{|c|c|c|c|c|c|c|}
\hline Index & Phylum & Family & Species & Host & Location & References \\
\hline 2686 & \multirow{26}{*}{ NEMATODA } & Anisakidae & Contracaecum radiatum & Chionodraco myersi & Weddell Sea & Klöser et.al. (1992) \\
\hline 2687 & & & Contracaecum radiatum & Cryodraco antarcticus & Weddell Sea & Klöser et.al. (1992) \\
\hline 2688 & & & Contracaecum radiatum & Gerlachea australis & Weddell Sea & Klöser et.al. (1992) \\
\hline 2689 & & & Contracaecum radiatum & Gobionotothen gibberifrons & South Shetland Islands & Palm et al. (2007) \\
\hline 2690 & & & Contracaecum radiatum & Lepidonotothen larseni & South Shetland Islands & Palm et al. (2007) \\
\hline 2691 & & & Contracaecum radiatum & Lepidonotothen nudifrons & South Shetland Islands & Palm et al. (2007) \\
\hline 2692 & & & Contracaecum radiatum & Lepidonotothen squamifrons & South Shetland Islands & Palm et al. (2007) \\
\hline 2693 & & & Contracaecum radiatum & Muraenolepis microps & South Shetland Islands & Palm et al. (2007) \\
\hline 2694 & & & Contracaecum radiatum & Notothenia coriiceps & $\begin{array}{l}\text { Potter Cove (King George } \\
\text { Island) }\end{array}$ & Palm et.al. (1998) \\
\hline 2695 & & & Contracaecum radiatum & Notothenia coriiceps & King George Island & Palm et.al. (1998) \\
\hline 2696 & & & Contracaecum radiatum & Parachaenichthys charcoti & South Shetland Islands & Palm et al. (2007) \\
\hline 2697 & & & Contracaecum radiatum & Pleurogramma antarcticus & Weddell Sea & Klöser et.al. (1992) \\
\hline 2698 & & & Contracaecum radiatum & Trematomus newnesi & $\begin{array}{l}\text { Adelie Land (Eastern } \\
\text { Antarctica) }\end{array}$ & Laskowski et.al. (2007) \\
\hline 2699 & & & Contracaecum radiatum & Trematomus eulepidotus & South Shetland Islands & Palm et al. (2007) \\
\hline 2700 & & & Contracaecum radiatum & Trematomus scotti & Weddell Sea & Klöser et.al. (1992) \\
\hline 2701 & & & Hysterothyiacium spp. & blank & blank & Ward \& Magath (1917) \\
\hline 2702 & & & Hysterothyiacium spp. & Dissostichus eleginoides & Shag Rocks & Brickle et al. (2005) \\
\hline 2703 & & & Hysterothyiacium spp. & Dissostichus eleginoides & South Georgia Island & Brickle et al. (2005) \\
\hline 2704 & & & Hysterothyiacium spp. & Dissostichus eleginoides & Prince Edward Island & Brickle et al. (2005) \\
\hline 2705 & & & Hysterothyiacium spp. & Dissostichus eleginoides & Heard Island & Brickle et al. (2005) \\
\hline 2706 & & & Hysterothyiacium spp. & Dissostichus eleginoides & Macquarie Island & Brickle et al. (2005) \\
\hline 2707 & & & Hysterothyiacium spp. & Dissostichus eleginoides & Ross Sea & Brickle et al. (2005) \\
\hline 2708 & & & Hysterothylacium aduncum & blank & blank & Rudolphi (1802) \\
\hline 2709 & & & Hysterothylacium aduncum & Dissostichus eleginoides & Falkland Patagonia & Gaevskaya et al. (1990) \\
\hline 2710 & & & Hysterothylacium aduncum & Dissostichus eleginoides & South Georgia Island & Gaevskaya et al. (1990) \\
\hline 2711 & & & Hysterothylacium aduncum & Macrourus carinatus & $\begin{array}{l}\text { Falkland (Malvinas) } \\
\text { Islands }\end{array}$ & Gaevskaya \& Rodyuk (1988) \\
\hline
\end{tabular}




\begin{tabular}{|c|c|c|c|c|c|c|}
\hline Index & Phylum & Family & Species & Host & Location & References \\
\hline 2712 & & & Hysterothylacium aduncum larva & blank & blank & Rudolphi (1802) \\
\hline 2713 & & & Hysterothylacium aduncum larva & Dissostichus eleginoides & Falkland-Patagonia & Gaevskaya et al. (1990) \\
\hline 2714 & & & Hysterothylacium aduncum larva & Macrourus carinatus & $\begin{array}{l}\text { Falkland (Malvinas) } \\
\text { Islands }\end{array}$ & Gaevskaya \& Rodyuk (1988) \\
\hline 2715 & \multirow{24}{*}{ NEMATODA } & Anisakidae & Hysterothylacium aduncum larva & Dissostichus eleginoides & Falkland Islands & Brickle et al. (2006) \\
\hline 2716 & & & Phocanema sp. & blank & blank & Myers (1959) \\
\hline 2717 & & & Phocanema sp. & Notothenia neglecta & Borge Bay & Hoogesteger \& White (1981) \\
\hline 2718 & & & Phocanema sp. & Notothenia neglecta & Signy Island & Hoogesteger \& White (1981) \\
\hline 2719 & & & Pseudanisakis sp. & Notothenia kempi & Shag Rocks & Parukhin \& Lyadov (1981) \\
\hline 2720 & & & Pseudoterranova sp. & blank & blank & Mosgovoy (1950) \\
\hline 2721 & & & Pseudoterranova sp. & Chaenocephalus aceratus & King George Island & Dzido et.al. (2009) \\
\hline 2722 & & & Pseudoterranova sp. & Notothenia rossii & King George Island & Dzido et.al. (2009) \\
\hline 2723 & & & Pseudoterranova sp. & Notothenia coriiceps & King George Island & Dzido et.al. (2009) \\
\hline 2724 & & & Pseudoterranova decipiens & blank & blank & Krabbe (1878) \\
\hline 2725 & & & Pseudoterranova decipiens & Acanthodraco dewitti & King George Island & Rokicki et. al. (2009) \\
\hline 2726 & & & Pseudoterranova decipiens & Acanthodraco dewitti & Admiralty Bay & Rokicki et. al. (2009) \\
\hline 2727 & & & Pseudoterranova decipiens & Artedidraco orianae & Weddell Sea & Palm (1999) \\
\hline 2728 & & & Pseudoterranova decipiens & Artedidraco skottsbergi & Weddell Sea & Palm (1999) \\
\hline 2729 & & & Pseudoterranova decipiens & Chaenocephalus aceratus & South Shetland Islands & Palm (1999) \\
\hline 2730 & & & Pseudoterranova decipiens & Chaenocephalus aceratus & South Shetland Islands & Palm et al. (2007) \\
\hline 2731 & & & Pseudoterranova decipiens & Chaenocephalus aceratus & Elephant Island & Rokicki et. al. (2009) \\
\hline 2732 & & & Pseudoterranova decipiens & Chaenocephalus aceratus & Shishkov Island & Rokicki et. al. (2009) \\
\hline 2733 & & & Pseudoterranova decipiens & Chaenocephalus aceratus & King George Island & Rokicki et. al. (2009) \\
\hline 2734 & & & Pseudoterranova decipiens & Chaenocephalus aceratus & Admiralty Bay & Rokicki et. al. (2009) \\
\hline 2735 & & & Pseudoterranova decipiens & Chaenodraco wilsoni & Elephant Island & Rokicki et. al. (2009) \\
\hline 2736 & & & Pseudoterranova decipiens & Chaenodraco wilsoni & Shishkov Island & Rokicki et. al. (2009) \\
\hline 2737 & & & Pseudoterranova decipiens & Champsocephalus gunnari & King George Island & Rokicki et. al. (2009) \\
\hline 2738 & & & Pseudoterranova decipiens & Champsocephalus gunnari & Admiralty Bay & Rokicki et. al. (2009) \\
\hline
\end{tabular}




\begin{tabular}{|c|c|c|c|c|c|c|}
\hline Index & Phylum & Family & Species & Host & Location & References \\
\hline 2739 & & & Pseudoterranova decipiens & Chiondraco rastrospinosus & South Shetland Islands & Palm (1999) \\
\hline 2740 & & & Pseudoterranova decipiens & Chiondraco rastrospinosus & Elephant Island & Rokicki et. al. (2009) \\
\hline 2741 & & & Pseudoterranova decipiens & Chiondraco rastrospinosus & Shishkov Island & Rokicki et. al. (2009) \\
\hline 2742 & & & Pseudoterranova decipiens & Chiondraco rastrospinosus & King George Island & Rokicki et. al. (2009) \\
\hline 2743 & & & Pseudoterranova decipiens & Chiondraco rastrospinosus & Admiralty Bay & Rokicki et. al. (2009) \\
\hline 2744 & \multirow{22}{*}{ NEMATODA } & Anisakidae & Pseudoterranova decipiens & Chionodraco hamatus & Adelie Land & Zdzitowiecki et.al. (1998) \\
\hline 2745 & & & Pseudoterranova decipiens & Chionodraco hamatus & Weddell Sea & Palm (1999) \\
\hline 2746 & & & Pseudoterranova decipiens & Chionodraco myersi & Weddell Sea & Palm (1999) \\
\hline 2747 & & & Pseudoterranova decipiens & Cryodraco antarcticus & Weddell Sea & Palm (1999) \\
\hline 2748 & & & Pseudoterranova decipiens & Cryodraco antarcticus & Elephant Island & Rokicki et. al. (2009) \\
\hline 2749 & & & Pseudoterranova decipiens & Cryodraco antarcticus & Shishkov Island & Rokicki et. al. (2009) \\
\hline 2750 & & & Pseudoterranova decipiens & Cryodraco antarcticus & King George Island & Rokicki et. al. (2009) \\
\hline 2751 & & & Pseudoterranova decipiens & Cryodraco antarcticus & Admiralty Bay & Rokicki et. al. (2009) \\
\hline 2752 & & & Pseudoterranova decipiens & Cygnodraco mawsoni & Weddell Sea & Klöser et.al. (1992) \\
\hline 2753 & & & Pseudoterranova decipiens & Dissostichus eleginoides & Shag Rocks & Brickle et al. (2005) \\
\hline 2754 & & & Pseudoterranova decipiens & Dissostichus eleginoides & South Georgia Island & Brickle et al. (2005) \\
\hline 2755 & & & Pseudoterranova decipiens & Dissostichus eleginoides & Prince Edward Island & Brickle et al. (2005) \\
\hline 2756 & & & Pseudoterranova decipiens & Dissostichus eleginoides & Heard Island & Brickle et al. (2005) \\
\hline 2757 & & & Pseudoterranova decipiens & Dissostichus eleginoides & Macquarie Island & Brickle et al. (2005) \\
\hline 2758 & & & Pseudoterranova decipiens & Dissostichus eleginoides & Ross Sea & Brickle et al. (2005) \\
\hline 2759 & & & Pseudoterranova decipiens & Dissostichus eleginoides & Falkland Islands & Brickle et al. (2006) \\
\hline 2760 & & & Pseudoterranova decipiens & Dissostichus mawsoni & Elephant Island & Rokicki et. al. (2009) \\
\hline 2761 & & & Pseudoterranova decipiens & Dissostichus mawsoni & Shishkov Island & Rokicki et. al. (2009) \\
\hline 2762 & & & Pseudoterranova decipiens & Dissostichus mawsoni & King George Island & Rokicki et. al. (2009) \\
\hline 2763 & & & Pseudoterranova decipiens & Dissostichus mawsoni & Admiralty Bay & Rokicki et. al. (2009) \\
\hline 2764 & & & Pseudoterranova decipiens & Dolliodraco largedorsalis & Weddell Sea & Palm (1999) \\
\hline 2765 & & & Pseudoterranova decipiens & Gobionotothen gibberifrons & South Shetland Islands & Palm (1999) \\
\hline
\end{tabular}




\begin{tabular}{|c|c|c|c|c|c|c|}
\hline Index & Phylum & Family & Species & Host & Location & References \\
\hline 2766 & & & Pseudoterranova decipiens & Gobionotothen gibberifrons & South Shetland Islands & Palm et al. (2007) \\
\hline 2767 & & & Pseudoterranova decipiens & Gobionotothen gibberifrons & Elephant Island & Rokicki et. al. (2009) \\
\hline 2768 & & & Pseudoterranova decipiens & Gobionotothen gibberifrons & Shishkov Island & Rokicki et. al. (2009) \\
\hline 2769 & & & Pseudoterranova decipiens & Gobionotothen gibberifrons & King George Island & Rokicki et. al. (2009) \\
\hline 2770 & & & Pseudoterranova decipiens & Gobionotothen gibberifrons & Admiralty Bay & Rokicki et. al. (2009) \\
\hline 2771 & & & Pseudoterranova decipiens & Harpagifer antarcticus & Vernadsky Station & $\begin{array}{c}\text { Laskowski \& Zdzitowiecki } \\
\text { (2005) }\end{array}$ \\
\hline 2772 & & & Pseudoterranova decipiens & Harpagifer georgianus & Admiralty Bay & Zdzitowiecki \& Zadrozny (1999) \\
\hline 2773 & & Anisakidae & Pseudoterranova decipiens & Harpagifer georgianus & Off Elephant Island & Zdzitowiecki \& Zadrozny (1999) \\
\hline 2774 & & & Pseudoterranova decipiens & Lepidonotothen kempi & Elephant Island & Rokicki et. al. (2009) \\
\hline 2775 & & & Pseudoterranova decipiens & Lepidonotothen kempi & Shishkov Island & Rokicki et. al. (2009) \\
\hline 2776 & & & Pseudoterranova decipiens & Lepidonotothen larseni & South Shetland Islands & Palm (1999) \\
\hline 2777 & & & Pseudoterranova decipiens & Lepidonotothen larseni & $\begin{array}{l}\text { Port Foster (Deception } \\
\text { Island) }\end{array}$ & Ruhl et al. (2003) \\
\hline 2778 & & & Pseudoterranova decipiens & Lepidonotothen larseni & South Shetland Islands & Palm et al. (2007) \\
\hline 2779 & & & Pseudoterranova decipiens & Lepidonotothen nudifrons & South Shetland Islands & Palm (1999) \\
\hline 2780 & & & Pseudoterranova decipiens & blank & blank & Palm et al. (2007) \\
\hline 2781 & & & Pseudoterranova decipiens & Lepidonotothen nudifrons & $\begin{array}{l}\text { Port Foster (Deception } \\
\text { Island) }\end{array}$ & Ruhl et al. (2003) \\
\hline 2782 & NEMATODA & & Pseudoterranova decipiens & Lepidonotothen nudifrons & King George Island & Rokicki et. al. (2009) \\
\hline 2783 & & & Pseudoterranova decipiens & Lepidonotothen nudifrons & Admiralty Bay & Rokicki et. al. (2009) \\
\hline 2784 & & & Pseudoterranova decipiens & Lepidonotothen squamifrons & South Shetland Islands & Palm (1999) \\
\hline 2785 & & & Pseudoterranova decipiens & blank & blank & Palm et al. (2007) \\
\hline 2786 & & & Pseudoterranova decipiens & Lindbergichthys nudifrons & Vernadsky Station & $\begin{array}{l}\text { Laskowski \& Zdzitowiecki } \\
\qquad(2005)\end{array}$ \\
\hline 2787 & & & Pseudoterranova decipiens & Lindbergichthys nudifrons & Admiralty Bay & $\begin{array}{l}\text { Laskowski \& Zdzitowiecki } \\
\text { (2005) }\end{array}$ \\
\hline 2788 & & & Pseudoterranova decipiens & Lycenchelus aratrirostris & Elephant Island & Rokicki et. al. (2009) \\
\hline 2789 & & & Pseudoterranova decipiens & Lycenchelus aratrirostris & Shishkov Island & Rokicki et. al. (2009) \\
\hline 2790 & & & Pseudoterranova decipiens & Muraenolepis microps & South Shetland Islands & Palm (1999) \\
\hline 2791 & & & Pseudoterranova decipiens & Muraenolepis microps & South Shetland Islands & Palm et al. (2007) \\
\hline
\end{tabular}




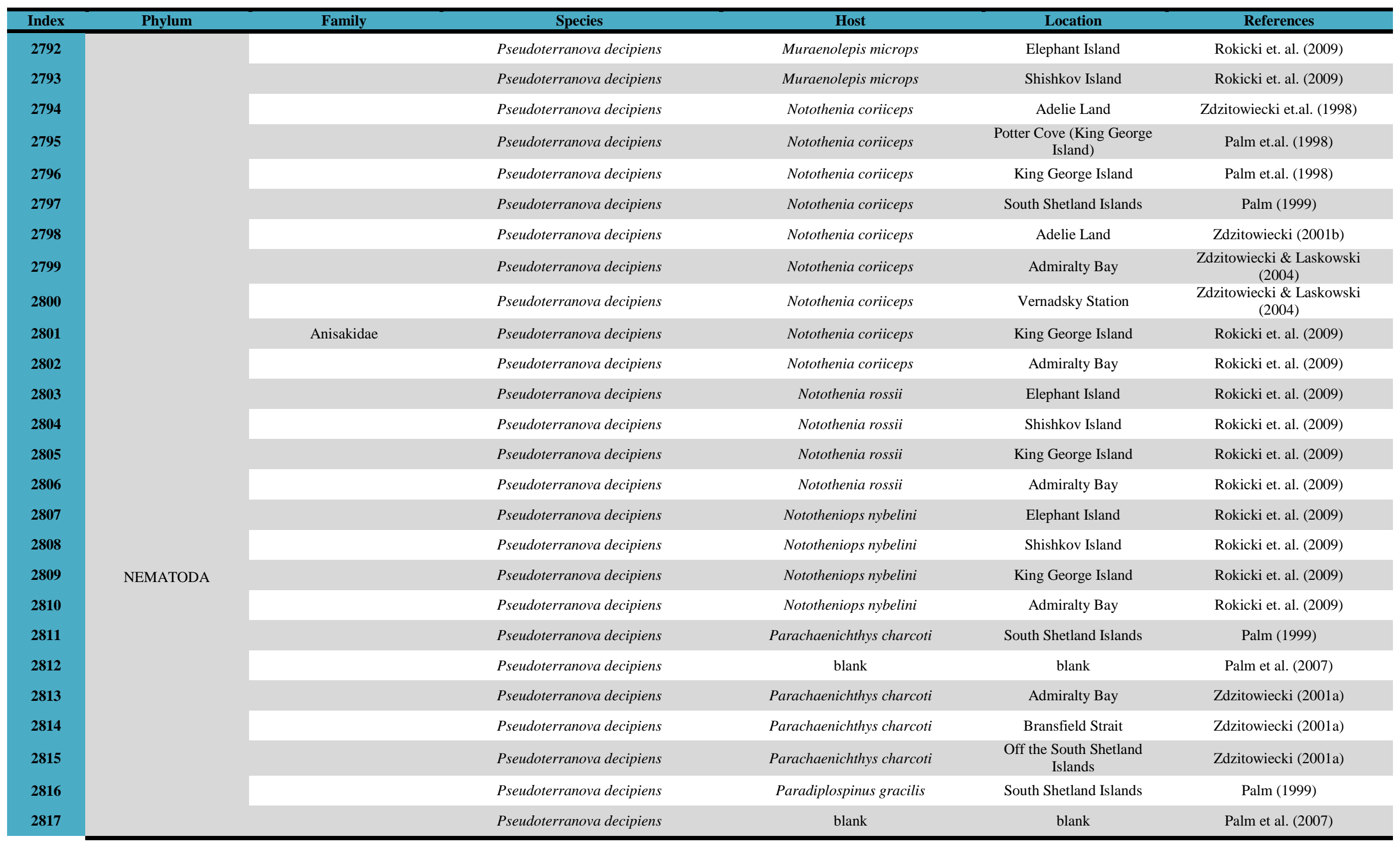




\begin{tabular}{|c|c|c|c|c|c|c|}
\hline Index & Phylum & Family & Species & Host & Location & References \\
\hline 2818 & & & Pseudoterranova decipiens & Pogonophryne marmorata & Elephant Island & Rokicki et. al. (2009) \\
\hline 2819 & & & Pseudoterranova decipiens & Pogonophryne marmorata & Shishkov Island & Rokicki et. al. (2009) \\
\hline 2820 & & & Pseudoterranova decipiens & Pseudochaenichthys georgianus & South Shetland Islands & Palm (1999) \\
\hline 2821 & & & Pseudoterranova decipiens & Trematomus bernacchii & Vernadsky Station & $\begin{array}{l}\text { Laskowski \& Zdzitowiecki } \\
\text { (2005) }\end{array}$ \\
\hline 2822 & & & Pseudoterranova decipiens & Trematomus bernacchii & Admiralty Bay & $\begin{array}{l}\text { Laskowski \& Zdzitowiecki } \\
\qquad(2005)\end{array}$ \\
\hline 2823 & & & Pseudoterranova decipiens & Trematomus bernacchii & King George Island & Rokicki et. al. (2009) \\
\hline 2824 & & & Pseudoterranova decipiens & Trematomus bernacchii & Admiralty Bay & Rokicki et. al. (2009) \\
\hline 2825 & & & Pseudoterranova decipiens & Trematomus eulepidotus & Weddell Sea & Palm (1999) \\
\hline 2826 & & & Pseudoterranova decipiens & Trematomus eulepidotus & South Shetland Islands & Palm (1999) \\
\hline 2827 & & & Pseudoterranova decipiens & Trematomus eulepidotus & South Shetland Islands & Palm et al. (2007) \\
\hline 2828 & & & Pseudoterranova decipiens & Trematomus eulepidotus & Elephant Island & Rokicki et. al. (2009) \\
\hline 2829 & & & Pseudoterranova decipiens & Trematomus eulepidotus & Shishkov Island & Rokicki et. al. (2009) \\
\hline 2830 & \multirow{14}{*}{ NEMATODA } & Anisakidae & Pseudoterranova decipiens & Trematomus hansoni & Adelie Land & Zdzitowiecki et.al. (1998) \\
\hline 2831 & & & Pseudoterranova decipiens & Trematomus hansoni & Weddell Sea & Palm (1999) \\
\hline 2832 & & & Pseudoterranova decipiens & Trematomus hansoni & King George Island & Rokicki et. al. (2009) \\
\hline 2833 & & & Pseudoterranova decipiens & Trematomus hansoni & Admiralty Bay & Rokicki et. al. (2009) \\
\hline 2834 & & & Pseudoterranova decipiens & Trematomus lepidorhinus & Elephant Island & Rokicki et. al. (2009) \\
\hline 2835 & & & Pseudoterranova decipiens & Trematomus lepidorhinus & Shishkov Island & Rokicki et. al. (2009) \\
\hline 2836 & & & Pseudoterranova decipiens & Trematomus lepidorhinus & Weddell Sea & Palm (1999) \\
\hline 2837 & & & Pseudoterranova decipiens & Trematomus loennbergi & Weddell Sea & Palm (1999) \\
\hline 2838 & & & Pseudoterranova decipiens & Trematomus newnesi & Vernadsky Station & $\begin{array}{l}\text { Laskowski \& Zdzitowiecki } \\
\qquad(2005)\end{array}$ \\
\hline 2839 & & & Pseudoterranova decipiens & Trematomus newnesi & Admiralty Bay & $\begin{array}{l}\text { Laskowski \& Zdzitowiecki } \\
\qquad(2005)\end{array}$ \\
\hline 2840 & & & Pseudoterranova decipiens & Trematomus newnesi & King George Island & Rokicki et. al. (2009) \\
\hline 2841 & & & Pseudoterranova decipiens & Trematomus newnesi & Admiralty Bay & Rokicki et. al. (2009) \\
\hline 2842 & & & Pseudoterranova decipiens & Trematomиs scotti & Weddell Sea & Klöser et.al. (1992) \\
\hline 2843 & & & Pseudoterranova decipiens & Trematomus scotti & Weddell Sea & Palm (1999) \\
\hline
\end{tabular}




\begin{tabular}{|c|c|c|c|c|c|c|}
\hline Index & Phylum & Family & Species & Host & Location & References \\
\hline 2844 & & & Pseudoterranova decipiens (larva) & blank & blank & Krabbe (1878) \\
\hline 2845 & & & Pseudoterranova decipiens (larva) & Dissostichus eleginoides & South Georgia Island & Gaevskaya et al. (1990) \\
\hline 2846 & & & Terranova sp. (larva) & blank & blank & Leiper \& Atkinson (1914) \\
\hline 2847 & & & Terranova sp. (larva) & HOST NAME NOT MENTIONED & Patagonian Shelf & Lyadov (1985) \\
\hline 2848 & & & Terranova sp. (larva) & HOST NAME NOT MENTIONED & Glacial Subregion & Lyadov (1985) \\
\hline 2849 & & & Terranova sp. (larva) & HOST NAME NOT MENTIONED & Kerguelen Subregion & Lyadov (1985) \\
\hline 2850 & & & Terranova sp. (larva) & HOST NAME NOT MENTIONED & South Australia & Lyadov (1985) \\
\hline 2851 & & & Terranova decipiens & blank & blank & Krabbe (1878) \\
\hline 2852 & & & Terranova decipiens & blank & blank & Baylis (1916) \\
\hline 2853 & & & Terranova decipiens & Chaenocephalus aceratus & Georgia & Parukhin \& Lyadov (1981) \\
\hline 2854 & & & Terranova decipiens & Notothenia rossii & Kerguelen Subregion & Parukhin \& Lyadov (1981) \\
\hline 2855 & & & Terranova decipiens & Notothenia rossii & Ob Bank & Parukhin \& Lyadov (1981) \\
\hline 2856 & & & Terranova decipiens & Notothenia rossii & Georgia & Parukhin \& Lyadov (1981) \\
\hline 2857 & & & Terranova decipiens & Notothenia rossii & Kerguelen Subregion & Parukhin \& Lyadov (1982) \\
\hline 2858 & & & Terranova decipiens & Notothenia rossii & Ob Bank & Parukhin \& Lyadov (1982) \\
\hline 2859 & \multirow{12}{*}{ NEMATODA } & Anisakidae & Terranova decipiens & Notothenia rossii rossii & Ob Bank & Parukhin (1986) \\
\hline 2860 & & & Terranova decipiens & Nototheniidae & Kerguelen Subregion & Parukhin \& Lyadov (1982) \\
\hline 2861 & & & Terranova decipiens & Pseudochaenichthys georgianus & Georgia & Parukhin \& Lyadov (1981) \\
\hline 2862 & & & Porroceacum sp. (Larvae) & Merluccius hubbsi & Patagonian Shelf & Reimer \& Jessen (1974) \\
\hline 2863 & & Cucullanidae & Cucullanellus fraseri & blank & blank & Baylis (1929) \\
\hline 2864 & & & Cucullanellus fraseri & blank & blank & Törnquist (1831) \\
\hline 2865 & & & Cucullanellus fraseri & Chaenichthys rhinoceratus & Kerguelen Subregion & Parukhin \& Lyadov (1981) \\
\hline 2866 & & & Cucullanellus fraseri & blank & blank & Lyadov et al. (1981) \\
\hline 2867 & & & Cucullanellus fraseri & Chaenichthys velifer & Kerguelen Subregion & Parukhin \& Lyadov (1981) \\
\hline 2868 & & & Cucullanellus fraseri & blank & blank & Lyadov et al. (1981) \\
\hline 2869 & & & Cucullanellus fraseri & Chaenocephalus aceratus & Georgia & Parukhin \& Lyadov (1981) \\
\hline 2870 & & & Cucullanellus fraseri & Champsocephalus gunnari & Kerguelen Subregion & Parukhin \& Lyadov (1981) \\
\hline
\end{tabular}




\begin{tabular}{|c|c|c|c|c|c|c|}
\hline Index & Phylum & Family & Species & Host & Location & References \\
\hline 2871 & & & Cucullanellus fraseri & blank & blank & Lyadov et al. (1981) \\
\hline 2872 & & & Cucullanellus fraseri & Dissostichus eleginoides & Kerguelen Subregion & Parukhin \& Lyadov (1981) \\
\hline 2873 & & & Cucullanellus fraseri & Dissostichus eleginoides & Crozet Islands & Parukhin \& Lyadov (1981) \\
\hline 2874 & & & Cucullanellus fraseri & Dissostichus eleginoides & Ob Bank & Parukhin \& Lyadov (1981) \\
\hline 2875 & & & Cucullanellus fraseri & Dissostichus eleginoides & Lena Bank & Parukhin \& Lyadov (1981) \\
\hline 2876 & & & Cucullanellus fraseri & Dissostichus eleginoides & Ob Bank & Parukhin (1986) \\
\hline 2877 & & & Cucullanellus fraseri & Dissostichus eleginoides & Lena Bank & Parukhin (1986) \\
\hline 2878 & & & Cucullanellus fraseri & Dissostichus eleginoides & Falkland Patagonia & Gaevskaya et al. (1990) \\
\hline 2879 & & & Cucullanellus fraseri & Dissostichus eleginoides & South Georgia Island & Gaevskaya et al. (1990) \\
\hline 2880 & & & Cucullanellus fraseri & Notothenia acuta & Kerguelen Subregion & Parukhin \& Lyadov (1981) \\
\hline 2881 & & & Cucullanellus fraseri & Notothenia gibberifrons & Georgia & Parukhin \& Lyadov (1981) \\
\hline 2882 & & & Cucullanellus fraseri & Notothenia kempi & Shag Rocks & Parukhin \& Lyadov (1981) \\
\hline 2883 & & & Cucullanellus fraseri & Notothenia larseni & Lena Bank & Parukhin (1986) \\
\hline 2884 & & & Cucullanellus fraseri & Notothenia magellanica & Ob Bank & Parukhin (1986) \\
\hline 2885 & & & Cucullanellus fraseri & Notothenia mizops & Kerguelen Subregion & Parukhin \& Lyadov (1981) \\
\hline 2886 & & & Cucullanellus fraseri & Notothenia rossii & Kerguelen Subregion & Parukhin \& Lyadov (1981) \\
\hline 2887 & & & Cucullanellus fraseri & Notothenia rossii & Kherd Island & Parukhin \& Lyadov (1981) \\
\hline 2888 & \multirow{10}{*}{ NEMATODA } & Cucullanidae & Cucullanellus fraseri & Notothenia rossii & Crozet Islands & Parukhin \& Lyadov (1981) \\
\hline 2889 & & & Cucullanellus fraseri & Notothenia rossii & Skif Bank & Parukhin \& Lyadov (1981) \\
\hline 2890 & & & Cucullanellus fraseri & Notothenia rossii & Ob Bank & Parukhin \& Lyadov (1981) \\
\hline 2891 & & & Cucullanellus fraseri & Notothenia rossii & Georgia & Parukhin \& Lyadov (1981) \\
\hline 2892 & & & Cucullanellus fraseri & Notothenia rossii rossii & Ob Bank & Parukhin (1986) \\
\hline 2893 & & & Cucullanellus fraseri & Notothenia rossii rossii & Lena Bank & Parukhin (1986) \\
\hline 2894 & & & Cucullanellus fraseri & Notothenia squamifrons & Kerguelen Subregion & Parukhin \& Lyadov (1981) \\
\hline 2895 & & & Cucullanellus fraseri & Notothenia squamifrons & Crozet Islands & Parukhin \& Lyadov (1981) \\
\hline 2896 & & & Cucullanellus fraseri & Notothenia squamifrons & Skif Bank & Parukhin \& Lyadov (1981) \\
\hline 2897 & & & Cucullanellus fraseri & Notothenia squamifrons & Ob Bank & Parukhin \& Lyadov (1981) \\
\hline
\end{tabular}




\begin{tabular}{|c|c|c|c|c|c|c|}
\hline Index & Phylum & Family & Species & Host & Location & References \\
\hline 2898 & & & Cucullanellus fraseri & Notothenia squamifrons & Lena Bank & Parukhin \& Lyadov (1981) \\
\hline 2899 & & & Cucullanellus fraseri & Pseudochaenichthys georgianus & Georgia & Parukhin \& Lyadov (1981) \\
\hline 2900 & & & Cucullanellus fraseri & HOST NAME NOT MENTIONED & Glacial Subregion & Lyadov (1985) \\
\hline 2901 & & & Cucullanellus fraseri & HOST NAME NOT MENTIONED & Kerguelen Subregion & Lyadov (1985) \\
\hline 2902 & & & Cucullanellus fraseri, var. nototheniae & blank & blank & Baylis (1929) \\
\hline 2903 & & & Cucullanellus fraseri, var. nototheniae & Dissostichus eleginoides & Kerguelen Subregion & Parukhin \& Lyadov (1982) \\
\hline 2904 & & & Cucullanellus fraseri, var. nototheniae & Dissostichus eleginoides & Skif Bank & Parukhin \& Lyadov (1982) \\
\hline 2905 & & & Cucullanellus fraseri, var. nototheniae & Dissostichus eleginoides & Ob Bank & Parukhin \& Lyadov (1982) \\
\hline 2906 & & & Cucullanellus fraseri, var. nototheniae & Dissostichus eleginoides & Lena Bank & Parukhin \& Lyadov (1982) \\
\hline 2907 & & & Cucullanellus fraseri, var. nototheniae & Notothenia acuta & Kerguelen Subregion & Parukhin \& Lyadov (1982) \\
\hline 2908 & & & Cucullanellus fraseri, var. nototheniae & Notothenia mizops & Kerguelen Subregion & Parukhin \& Lyadov (1982) \\
\hline 2909 & & & Cucullanellus fraseri, var. nototheniae & Notothenia rossii & Kerguelen Subregion & Parukhin \& Lyadov (1982) \\
\hline 2910 & & & Cucullanellus fraseri, var. nototheniae & Notothenia rossii & Kherd Island & Parukhin \& Lyadov (1982) \\
\hline 2911 & & & Cucullanellus fraseri, var. nototheniae & Notothenia rossii & Crozet Islands & Parukhin \& Lyadov (1982) \\
\hline 2912 & & & Cucullanellus fraseri, var. nototheniae & Notothenia rossii & Skif Bank & Parukhin \& Lyadov (1982) \\
\hline 2913 & & & Cucullanellus fraseri, var. nototheniae & Notothenia rossii & Ob Bank & Parukhin \& Lyadov (1982) \\
\hline 2914 & & & Cucullanellus fraseri, var. nototheniae & Notothenia squamifrons & Kerguelen Subregion & Parukhin \& Lyadov (1982) \\
\hline 2915 & & & Cucullanellus fraseri, var. nototheniae & Notothenia squamifrons & Crozet Islands & Parukhin \& Lyadov (1982) \\
\hline 2916 & & & Cucullanellus fraseri, var. nototheniae & Notothenia squamifrons & Skif Bank & Parukhin \& Lyadov (1982) \\
\hline 2917 & \multirow{8}{*}{ NEMATODA } & Cucullanidae & Cucullanellus fraseri, var. nototheniae & Notothenia squamifrons & Ob Bank & Parukhin \& Lyadov (1982) \\
\hline 2918 & & & Cucullanellus fraseri, var. nototheniae & Notothenia squamifrons & Lena Bank & Parukhin \& Lyadov (1982) \\
\hline 2919 & & & Dichelyne (Cucullaneilus) fraseri & blank & blank & Baylis (1929) \\
\hline 2920 & & & Dichelyne (Cucullaneilus) fraseri & Dissostichus eleginoides & South Georgia Island & Brickle et al. (2005) \\
\hline 2921 & & & Dichelyne (Cucullaneilus) fraseri & Dissostichus eleginoides & Prince Edward Island & Brickle et al. (2005) \\
\hline 2922 & & & Dichelyne (Cucullaneilus) fraseri & Dissostichus eleginoides & Heard Island & Brickle et al. (2005) \\
\hline 2923 & & & Dichelyne (Cucullaneilus) fraseri & Dissostichus eleginoides & Macquarie Island & Brickle et al. (2005) \\
\hline 2924 & & & Dichelyne (Cucullaneilus) fraseri & Dissostichus eleginoides & Falkland Islands & Brickle et al. (2006) \\
\hline
\end{tabular}




\begin{tabular}{|c|c|c|c|c|c|c|}
\hline Index & Phylum & Family & Species & Host & Location & References \\
\hline 2925 & & & Dichelyne (Cucullaneilus) fraseri & Nototheniidae & Kerguelen Subregion & Parukhin \& Lyadov (1982) \\
\hline 2926 & & Cystidicolidae & Ascarophis sp. (Larvae) & Dissostichus eleginoides & Ob Bank & Parukhin (1986) \\
\hline 2927 & & & Ascarophis morrhuae & blank & blank & Beneden (1870) \\
\hline 2928 & & & Ascarophis morrhuae & Champsocephalus gunnari & Georgia & Parukhin \& Lyadov (1981) \\
\hline 2929 & & & Ascarophis morrhuae & Notothenia acuta & Kerguelen Subregion & Parukhin \& Lyadov (1982) \\
\hline 2930 & & & Ascarophis morrhuae & Notothenia mizops & Kerguelen Subregion & Parukhin \& Lyadov (1982) \\
\hline 2931 & & & Ascarophis morrhuae & blank & blank & Parukhin \& Lyadov (1981) \\
\hline 2932 & & & Ascarophis morrhuae & Notothenia rossii & Georgia & Parukhin \& Lyadov (1981) \\
\hline 2933 & & & Ascarophis morrhuae & Notothenia squamifrons & Kerguelen Subregion & Parukhin \& Lyadov (1982) \\
\hline 2934 & & & Ascarophis morrhuae & Notothenia squamifrons & Crozet Islands & Parukhin \& Lyadov (1982) \\
\hline 2935 & & & Ascarophis morrhuae & Notothenia squamifrons & Skif Bank & Parukhin \& Lyadov (1982) \\
\hline 2936 & & & Ascarophis morrhuae & Notothenia squamifrons & Ob Bank & Parukhin \& Lyadov (1982) \\
\hline 2937 & & & Ascarophis morrhuae & Notothenia squamifrons & Lena Bank & Parukhin \& Lyadov (1982) \\
\hline 2938 & & & Ascarophis nototheniae & blank & blank & Johnston \& Mawson (1945) \\
\hline 2939 & & & Ascarophis nototheniae & Chaenocephalus aceratus & South Shetland Islands & Rocka (1999) \\
\hline 2940 & & & Ascarophis nototheniae & Chaenocephalus aceratus & South Georgia Island & Rocka (1999) \\
\hline 2941 & & & Ascarophis nototheniae & Chaenocephalus aceratus & South Orkney Islands & Rocka (1999) \\
\hline 2942 & & & Ascarophis nototheniae & Chaenocephalus aceratus & South Shetland Islands & Palm et al. (2007) \\
\hline 2943 & & & Ascarophis nototheniae & Chionodraco hamatus & Adelie Land & Zdzitowiecki et.al. (1998) \\
\hline 2944 & & & Ascarophis nototheniae & blank & blank & Zdzitowiecki (2001b) \\
\hline 2945 & & & Ascarophis nototheniae & Cryodraco antarcticus & South Shetland Islands & Rocka (1999) \\
\hline 2946 & \multirow{6}{*}{ NEMATODA } & Cystidicolidae & Ascarophis nototheniae & Cryodraco antarcticus & South Georgia Island & Rocka (1999) \\
\hline 2947 & & & Ascarophis nototheniae & Cryodraco antarcticus & South Orkney Islands & Rocka (1999) \\
\hline 2948 & & & Ascarophis nototheniae & Dissostichus eleginoides & South Georgia Island & Gaevskaya et al. (1990) \\
\hline 2949 & & & Ascarophis nototheniae & Dissostichus eleginoides & Shag Rocks & Brickle et al. (2005) \\
\hline 2950 & & & Ascarophis nototheniae & Dissostichus eleginoides & South Georgia Island & Brickle et al. (2005) \\
\hline 2951 & & & Ascarophis nototheniae & Dissostichus eleginoides & Prince Edward Island & Brickle et al. (2005) \\
\hline
\end{tabular}




\begin{tabular}{|c|c|c|c|c|c|c|}
\hline Index & Phylum & Family & Species & Host & Location & References \\
\hline 2952 & & & Ascarophis nototheniae & Dissostichus eleginoides & Heard Island & Brickle et al. (2005) \\
\hline 2953 & & & Ascarophis nototheniae & Dissostichus eleginoides & Macquarie Island & Brickle et al. (2005) \\
\hline 2954 & & & Ascarophis nototheniae & Dissostichus eleginoides & Falkland Islands & Brickle et al. (2006) \\
\hline 2955 & & & Ascarophis nototheniae & Gobionotothen gibberifrons & South Shetland Islands & Palm et al. (2007) \\
\hline 2956 & & & Ascarophis nototheniae & Gymnodraco acuticeps & Ross Sea & Laskowski et.al. (2005) \\
\hline 2957 & & & Ascarophis nototheniae & Harpagifer georgianus & Admiralty Bay & Zdzitowiecki \& Zadrozny (1999) \\
\hline 2958 & & & Ascarophis nototheniae & Lepidonotothen larseni & South Shetland Islands & Palm et al. (2007) \\
\hline 2959 & & & Ascarophis nototheniae & Lepidonotothen nudifrons & South Shetland Islands & Palm et al. (2007) \\
\hline 2960 & & & Ascarophis nototheniae & Lepidonotothen squamifrons & South Shetland Islands & Palm et al. (2007) \\
\hline 2961 & & & Ascarophis nototheniae & Lindbergichthys nudifrons & Vernadsky Station & $\begin{array}{c}\text { Laskowski \& Zdzitowiecki } \\
\text { (2005) }\end{array}$ \\
\hline 2962 & & & Ascarophis nototheniae & Lindbergichthys nudifrons & Admiralty Bay & $\begin{array}{l}\text { Laskowski \& Zdzitowiecki } \\
\text { (2005) }\end{array}$ \\
\hline 2963 & & & Ascarophis nototheniae & Macrourus carinatus & $\begin{array}{l}\text { Falkland (Malvinas) } \\
\text { Islands }\end{array}$ & Gaevskaya \& Rodyuk (1988) \\
\hline 2964 & & & Ascarophis nototheniae & Notothenia acuta & Kerguelen Subregion & Parukhin \& Lyadov (1981) \\
\hline 2965 & & & Ascarophis nototheniae & Notothenia coriiceps & South Shetland Islands & Palm et.al. (1998) \\
\hline 2966 & & & Ascarophis nototheniae & Notothenia coriiceps & Adelie Land & Zdzitowiecki et.al. (1998) \\
\hline 2967 & & & Ascarophis nototheniae & blank & blank & Zdzitowiecki (2001b) \\
\hline 2968 & & & Ascarophis nototheniae & Notothenia coriiceps & Admiralty Bay & $\begin{array}{l}\text { Zdzitowiecki \& Laskowski } \\
\text { (2004) }\end{array}$ \\
\hline 2969 & & & Ascarophis nototheniae & Notothenia coriiceps & Vernadsky Station & $\begin{array}{l}\text { Zdzitowiecki \& Laskowski } \\
\text { (2004) }\end{array}$ \\
\hline 2970 & & & Ascarophis nototheniae & Notothenia rossii & Georgia & Parukhin \& Lyadov (1981) \\
\hline 2971 & & & Ascarophis nototheniae & Notothenia squamifrons & Kerguelen Subregion & Parukhin \& Lyadov (1981) \\
\hline 2972 & & & Ascarophis nototheniae & Notothenia squamifrons & Crozet Islands & Parukhin \& Lyadov (1981) \\
\hline 2973 & & & Ascarophis nototheniae & Notothenia squamifrons & Skif Bank & Parukhin \& Lyadov (1981) \\
\hline 2974 & \multirow{4}{*}{ NEMATODA } & Cystidicolidae & Ascarophis nototheniae & Notothenia squamifrons & Ob Bank & Parukhin \& Lyadov (1981) \\
\hline 2975 & & & Ascarophis nototheniae & Notothenia squamifrons & Lena Bank & Parukhin \& Lyadov (1981) \\
\hline 2976 & & & Ascarophis nototheniae & Notothenia squamifrons & Georgia & Parukhin \& Lyadov (1981) \\
\hline 2977 & & & Ascarophis nototheniae & Notothenia squamifrons & Lena Bank & Parukhin (1986) \\
\hline
\end{tabular}




\begin{tabular}{|c|c|c|c|c|c|c|}
\hline Index & Phylum & Family & Species & Host & Location & References \\
\hline 2978 & & & Ascarophis nototheniae & Nototheniidae & Kerguelen Subregion & Parukhin \& Lyadov (1982) \\
\hline 2979 & & & Ascarophis nototheniae & Parachaenichthys charcoti & Admiralty Bay & Zdzitowiecki (2001a) \\
\hline 2980 & & & Ascarophis nototheniae & Parachaenichthys charcoti & $\begin{array}{l}\text { Off the South Shetland } \\
\text { Islands }\end{array}$ & Zdzitowiecki (2001a) \\
\hline 2981 & & & Ascarophis nototheniae & Parachaenichthys charcoti & $\begin{array}{l}\text { Admiralty Bay Bransfield } \\
\text { Strait }\end{array}$ & Zdzitowiecki (2001a) \\
\hline 2982 & & & Ascarophis nototheniae & Parachaenichthys charcoti & South Shetland Islands & Palm et al. (2007) \\
\hline 2983 & & & Ascarophis nototheniae & Trematomus newnesi & Vernadsky Station & $\begin{array}{l}\text { Laskowski \& Zdzitowiecki } \\
\qquad(2005)\end{array}$ \\
\hline 2984 & & & Ascarophis nototheniae & Trematomus bernacchii & Mcmurdo Sound & Moser \& Cowen (1991) \\
\hline 2985 & & & Ascarophis nototheniae & Trematomus bernacchii & Adelie Land & Zdzitowiecki et.al. (1998) \\
\hline 2986 & & & Ascarophis nototheniae & Trematomus bernacchii & Admiralty Bay & $\begin{array}{l}\text { Laskowski \& Zdzitowiecki } \\
\text { (2005) }\end{array}$ \\
\hline 2987 & & & Ascarophis nototheniae & Trematomus bernacchii & Vernadsky Station & $\begin{array}{l}\text { Laskowski \& Zdzitowiecki } \\
\text { (2005) }\end{array}$ \\
\hline 2988 & & & Ascarophis nototheniae & Trematomus hansoni & Adelie Land & Zdzitowiecki et.al. (1998) \\
\hline 2989 & & & Ascarophis nototheniae & HOST NAME NOT MENTIONED & Patagonian Shelf & Lyadov (1985) \\
\hline 2990 & & & Ascarophis nototheniae & HOST NAME NOT MENTIONED & Glacial Subregion & Lyadov (1985) \\
\hline 2991 & & & Ascarophis nototheniae & HOST NAME NOT MENTIONED & Kerguelen Subregion & Lyadov (1985) \\
\hline 2992 & & & Ascarophis nototheniae & HOST NAME NOT MENTIONED & South Australia & Lyadov (1985) \\
\hline 2993 & & & $\begin{array}{c}\text { Cystidicola (Rhabdochona) } \\
\text { beatriceinsleyae }\end{array}$ & blank & blank & Holloway \& Klewer (1969) \\
\hline 2994 & & & $\begin{array}{c}\text { Cystidicola (Rhabdochona) } \\
\text { beatriceinsleyae }\end{array}$ & Rhigophila (Lycodichthys) dearborni & Mcmurdo Sound & Holloway \& Klewer (1969) \\
\hline 2995 & & & Cystidicola marina & blank & blank & Szidat (1961) \\
\hline 2996 & & & Cystidicola marina & Eleginops maclovinus & South Shetland Islands & Szidat \& Graefe (1967) \\
\hline 2997 & & & Cystidicola marina & Parachaenichthys charcoti & South Shetland Islands & Szidat \& Graefe (1967) \\
\hline 2998 & & & Neoascarophis sp. & blank & blank & Machida (1976) \\
\hline 2999 & & & Neoascarophis sp. & Macrourus carinatus & $\begin{array}{l}\text { Falkland (Malvinas) } \\
\text { Islands }\end{array}$ & Gaevskaya \& Rodyuk (1988) \\
\hline 3000 & & & Philometra sp. & blank & blank & Gaevskaya \& Rodyuk (1988) \\
\hline 3001 & & & Philometra sp. & blank & blank & Costa (1845) \\
\hline 3002 & NEMATODA & Cystidicolidae & Philometra sp. & Macrourus carinatus & $\begin{array}{l}\text { Falkland (Malvinas) } \\
\text { Islands }\end{array}$ & Gaevskaya \& Rodyuk (1988) \\
\hline 3003 & & Rhabdochonidae & Rhabdochona beatriceinsleyae & blank & blank & Holloway \& Klewer (1969) \\
\hline
\end{tabular}




\begin{tabular}{|c|c|c|c|c|c|c|}
\hline Index & Phylum & Family & Species & Host & Location & References \\
\hline 3004 & & & Rhabdochona beatriceinsleyae & Rhigophila dearborni & Mcmurdo Sound & Holloway \& Klewer (1969) \\
\hline 3005 & & Trichuridae & Capillaria $s p$. & blank & blank & Zeder (1800) \\
\hline 3006 & & & Capillaria sp. & Dissostichus eleginoides & South Georgia Island & Brickle et al. (2005) \\
\hline 3007 & & & Capillaria sp. & Dissostichus eleginoides & Prince Edward Island & Brickle et al. (2005) \\
\hline 3008 & & & Capillaria sp. & Dissostichus eleginoides & Macquarie Island & Brickle et al. (2005) \\
\hline 3009 & & & Capillaria sp. & Macrourus carinatus & $\begin{array}{l}\text { Falkland (Malvinas) } \\
\text { Islands }\end{array}$ & Gaevskaya \& Rodyuk (1988) \\
\hline 3010 & \multirow{20}{*}{ ANNELIDA } & blank & Hirudinea gen. sp. & Chaenocephalus aceratus & Georgia & Parukhin \& Lyadov (1981) \\
\hline 3011 & & & Hirudinea gen. sp. & Champsocephalus gunnari & Georgia & Parukhin \& Lyadov (1981) \\
\hline 3012 & & & Hirudinea gen. sp. & Notothenia rossi & Georgia & Parukhin \& Lyadov (1981) \\
\hline 3013 & & & Hirudinea gen. sp. & Pseudochaenichthys georgianus & Georgia & Parukhin \& Lyadov (1981) \\
\hline 3014 & & & Hirudinea sp. & Lepidonotothen squamifrons & Antarctica & Rohde et al. (1995) \\
\hline 3015 & & & Lernaeidae sp. & Macrourus holotrachys & Antarctica & Rohde et al. (1995) \\
\hline 3016 & & Piscicolidae & Antarcticobdella crozetensis & blank & blank & Sawyer (1972) \\
\hline 3017 & & & Antarcticobdella crozetensis & Notothenia coriiceps & $\begin{array}{l}\text { Ile Possession of The } \\
\text { Crozet Island }\end{array}$ & Sawyer (1972) \\
\hline 3018 & & & Antarcticobdella crozetensis & Notothenia coriiceps & Marion Island & Sawyer \& Villier (1976) \\
\hline 3019 & & & Antarcticobdella crozetensis & Notothenia coriiceps & Crozet Islands & Sawyer \& Villier (1976) \\
\hline 3020 & & & Antarctobdella tcherniai & blank & blank & Dollfus (1964) \\
\hline 3021 & & & Antarctobdella tcherniai & Notothenia neglecta & Borge Bay & Hoogesteger \& White (1981) \\
\hline 3022 & & & Antarctobdella tcherniai & Notothenia neglecta & Signy Island & Hoogesteger \& White (1981) \\
\hline 3023 & & & Austrobdella translucens & blank & blank & Badham (1916) \\
\hline 3024 & & & Austrobdella translucens & Notothenia sp. & $\begin{array}{c}\text { Kerguelen Subregion } \\
\text { Islands }\end{array}$ & Utevsky (2005) \\
\hline 3025 & & & Austrobdella translucens & Chaenocephalus sp. & $\begin{array}{c}\text { Kerguelen Subregion } \\
\text { Islands }\end{array}$ & Utevsky (2005) \\
\hline 3026 & & & Cryobdella sp. & blank & blank & Brinkmann, Jr. (1948) \\
\hline 3027 & & & Cryobdella sp. & Notothenia squamifrons & Crozet Islands & Parukhin \& Lyadov (1981) \\
\hline 3028 & & & Cryobdella sp. & Notothenia squamifrons & Crozet Islands & Parukhin \& Lyadov (1982) \\
\hline 3029 & & & Cryobdella antarctica & blank & blank & Epstein (1970) \\
\hline
\end{tabular}




\begin{tabular}{|c|c|c|c|c|c|c|}
\hline Index & Phylum & Family & Species & Host & Location & References \\
\hline 3030 & \multirow{26}{*}{ ANNELIDA } & Piscicolidae & Cryobdella antarctica & Trematomus bernacchii & Davis Sea & Utevsky (2005) \\
\hline 3031 & & & Cryobdella antarctica & Trematomus bernacchii & Scottia Sea & Utevsky (2005) \\
\hline 3032 & & & Cryobdella levigata & blank & blank & Harding (1923) \\
\hline 3033 & & & Cryobdella levigata & Trematomus hansoni & Ross Sea (Victoria Land) & Utevsky (2005) \\
\hline 3034 & & & Cryobdella levigata & Trematomus hansoni & Davis Sea & Utevsky (2005) \\
\hline 3035 & & & Cryobdella levigata & Trematomus hansoni & Mawson Sea & Utevsky (2005) \\
\hline 3036 & & & Cryobdella levigata & Trematomus hansoni & $\begin{array}{l}\text { Kerguelen Subregion } \\
\text { Islands }\end{array}$ & Utevsky (2005) \\
\hline 3037 & & & Cryobdella levigata & Trematomus bernacchii & Ross Sea (Victoria Land) & Utevsky (2005) \\
\hline 3038 & & & Cryobdella levigata & Trematomus bernacchii & Davis Sea & Utevsky (2005) \\
\hline 3039 & & & Cryobdella levigata & Trematomus bernacchii & Mawson Sea & Utevsky (2005) \\
\hline 3040 & & & Cryobdella levigata & Trematomus bernacchii & $\begin{array}{l}\text { Kerguelen Subregion } \\
\text { Islands }\end{array}$ & Utevsky (2005) \\
\hline 3041 & & & Cryobdella ljadovi & blank & blank & Epshtein \& Utevsky (1994) \\
\hline 3042 & & & Cryobdella ljadovi & Muraenolepis marmoratus & $\begin{array}{l}\text { Kerguelen Subregion } \\
\text { Coastal Waters }\end{array}$ & Utevsky (2005) \\
\hline 3043 & & & Cryobdella ljadovi & Muraenolepis microps & South Orkney Island & Utevsky (2005) \\
\hline 3044 & & & Cryobdella pallida & blank & blank & Utevsky (1997) \\
\hline 3045 & & & Cryobdella pallida & Notothenia squamifrons & Crozet Islands & Utevsky (2005) \\
\hline 3046 & & & Epsteinia alba & blank & blank & Epstein (1970) \\
\hline 3047 & & & Epsteinia alba & Trematomus borchgrevinski & Enderby Land & Utevsky (2005) \\
\hline 3048 & & & Glyptonotobdella antarctica & blank & blank & Sawyer \& White (1969) \\
\hline 3049 & & & Glyptonotobdella antarctica & Chaenocephalus aceratus & S.Orkney Island & Utevsky (2005) \\
\hline 3050 & & & Glyptonotobdella antarctica & Chaenocephalus aceratus & S.Sandwich Island & Utevsky (2005) \\
\hline 3051 & & & Glyptonotobdella antarctica & Chaenocephalus aceratus & Scottia Sea & Utevsky (2005) \\
\hline 3052 & & & Glyptonotobdella antarctica & Chaenocephalus aceratus & Princess Martha Coast & Utevsky (2005) \\
\hline 3053 & & & Glyptonotobdella antarctica & Chaenocephalus aceratus & Mcmurdo Sound & Utevsky (2005) \\
\hline 3054 & & & Glyptonotobdella antarctica & Chaenocephalus aceratus & Ross Sea & Utevsky (2005) \\
\hline 3055 & & & Glyptonotobdella antarctica & Chaenocephalus aceratus & Marion Island & Utevsky (2005) \\
\hline
\end{tabular}




\begin{tabular}{|c|c|c|c|c|c|c|}
\hline Index & Phylum & Family & Species & Host & Location & References \\
\hline 3056 & & & Glyptonotobdella antarctica & Chionodraco sp. & S.Orkney Island & Utevsky (2005) \\
\hline 3057 & & & Glyptonotobdella antarctica & Chionodraco sp. & S.Sandwich Island & Utevsky (2005) \\
\hline 3058 & & & Glyptonotobdella antarctica & Chionodraco sp. & Scottia Sea & Utevsky (2005) \\
\hline 3059 & & Piscicolidae & Glyptonotobdella antarctica & Chionodraco sp. & Princess Martha Coast & Utevsky (2005) \\
\hline 3060 & & & Glyptonotobdella antarctica & Chionodraco sp. & Mcmurdo Sound & Utevsky (2005) \\
\hline 3061 & & & Glyptonotobdella antarctica & Chionodraco sp. & Ross Sea & Utevsky (2005) \\
\hline 3062 & & & Glyptonotobdella antarctica & Chionodraco sp. & Marion Island & Utevsky (2005) \\
\hline 3063 & & & Ichthyobdella tentaculata & blank & blank & Cordero (1937) \\
\hline 3064 & & & Ichthyobdella tentaculata & Notothenia neglecta & $\begin{array}{c}\text { Bhaia Scotia La Isla } \\
\text { Laurie (Orcadas del sur, } \\
\text { Argentina) }\end{array}$ & Szidat (1965) \\
\hline 3065 & & & Notobdella nototheniae & blank & blank & Benham (1901) \\
\hline 3066 & & & Notobdella nototheniae & Notothenia sp. & $\begin{array}{l}\text { Bhaia Scotia La Isla } \\
\text { Laurie (Orcadas del sur, } \\
\text { Argentina) }\end{array}$ & Szidat (1965) \\
\hline 3067 & & & Notobdella nototheniae & Notothenia coriiceps & $\begin{array}{l}\text { Potter Cove (King George } \\
\text { Island) }\end{array}$ & Palm et.al. (1998) \\
\hline 3068 & ANNELIDA & & Notobdella nototheniae & Parachaenichthys georgianus & $\begin{array}{c}\text { Bhaia Scotia La Isla } \\
\text { Laurie (Orcadas del sur, } \\
\text { Argentina) }\end{array}$ & Szidat (1965) \\
\hline 3069 & & & Nototheniobdella sawyeri & blank & blank & Utevsky (1993) \\
\hline 3070 & & & Nototheniobdella sawyeri & Chaenodraco wilsoni & Palmer Coast & Utevsky (2005) \\
\hline 3071 & & & Nototheniobdella sawyeri & Chaenodraco wilsoni & South Shetland Islands & Utevsky (2005) \\
\hline 3072 & & & Nototheniobdella sawyeri & Chaenodraco wilsoni & $\begin{array}{l}\text { Filchner Ice Shelf } \\
\text { (Weddell Sea) }\end{array}$ & Utevsky (2005) \\
\hline 3073 & & & Nototheniobdella sawyeri & Chaenodraco wilsoni & Clarie Coast & Utevsky (2005) \\
\hline 3074 & & & Nototheniobdella sawyeri & Chaenodraco wilsoni & Scott Coast & Utevsky (2005) \\
\hline 3075 & & & Nototheniobdella sawyeri & Chaenodraco wilsoni & $\begin{array}{l}\text { Franklin Island (Ross } \\
\text { Sea) }\end{array}$ & Utevsky (2005) \\
\hline 3076 & & & Nototheniobdella sawyeri & Chionodraco hamatus & Palmer Coast & Utevsky (2005) \\
\hline 3077 & & & Nototheniobdella sawyeri & Chionodraco hamatus & South Shetland Islands & Utevsky (2005) \\
\hline 3078 & & & Nototheniobdella sawyeri & Chionodraco hamatus & $\begin{array}{l}\text { Filchner Ice Shelf } \\
\text { (Weddell Sea) }\end{array}$ & Utevsky (2005) \\
\hline 3079 & & & Nototheniobdella sawyeri & Chionodraco hamatus & Clarie Coast & Utevsky (2005) \\
\hline
\end{tabular}




\begin{tabular}{|c|c|c|c|c|c|c|}
\hline Index & Phylum & Family & Species & Host & Location & References \\
\hline 3080 & & & Nototheniobdella sawyeri & Chionodraco hamatus & Scott Coast & Utevsky (2005) \\
\hline 3081 & & & Nototheniobdella sawyeri & Chionodraco hamatus & $\begin{array}{c}\text { Franklin Island (Ross } \\
\text { Sea) }\end{array}$ & Utevsky (2005) \\
\hline 3082 & & & Nototheniobdella sawyeri & Chionodraco kathleenae & Palmer Coast & Utevsky (2005) \\
\hline 3083 & & & Nototheniobdella sawyeri & Chionodraco kathleenae & South Shetland Islands & Utevsky (2005) \\
\hline 3084 & & & Nototheniobdella sawyeri & Chionodraco kathleenae & $\begin{array}{l}\text { Filchner Ice Shelf } \\
\text { (Weddell Sea) }\end{array}$ & Utevsky (2005) \\
\hline 3085 & & Piscicolidae & Nototheniobdella sawyeri & Chionodraco kathleenae & Clarie Coast & Utevsky (2005) \\
\hline 3086 & & & Nototheniobdella sawyeri & Chionodraco kathleenae & Scott Coast & Utevsky (2005) \\
\hline 3087 & & & Nototheniobdella sawyeri & Chionodraco kathleenae & $\begin{array}{c}\text { Franklin Island (Ross } \\
\text { Sea) }\end{array}$ & Utevsky (2005) \\
\hline 3088 & & & Nototheniobdella sawyeri & Cryodraco antarcticus & Palmer Coast & Utevsky (2005) \\
\hline 3089 & & & Nototheniobdella sawyeri & Cryodraco antarcticus & South Shetland Islands & Utevsky (2005) \\
\hline 3090 & & & Nototheniobdella sawyeri & Cryodraco antarcticus & $\begin{array}{l}\text { Filchner Ice Shelf } \\
\text { (Weddell Sea) }\end{array}$ & Utevsky (2005) \\
\hline 3091 & & & Nototheniobdella sawyeri & Cryodraco antarcticus & Clarie Coast & Utevsky (2005) \\
\hline 3092 & & & Nototheniobdella sawyeri & Cryodraco antarcticus & Scott Coast & Utevsky (2005) \\
\hline 3093 & & & Nototheniobdella sawyeri & Cryodraco antarcticus & $\begin{array}{c}\text { Franklin Island (Ross } \\
\text { Sea) }\end{array}$ & Utevsky (2005) \\
\hline 3094 & & & Nototheniobdella sawyeri & Neopagetopsis ionah & Palmer Coast & Utevsky (2005) \\
\hline 3095 & ANNELIDA & & Nototheniobdella sawyeri & Neopagetopsis ionah & South Shetland Islands & Utevsky (2005) \\
\hline 3096 & & & Nototheniobdella sawyeri & Neopagetopsis ionah & $\begin{array}{l}\text { Filchner Ice Shelf } \\
\text { (Weddell Sea) }\end{array}$ & Utevsky (2005) \\
\hline 3097 & & & Nototheniobdella sawyeri & Neopagetopsis ionah & Clarie Coast & Utevsky (2005) \\
\hline 3098 & & & Nototheniobdella sawyeri & Neopagetopsis ionah & Scott Coast & Utevsky (2005) \\
\hline 3099 & & & Nototheniobdella sawyeri & Neopagetopsis ionah & $\begin{array}{c}\text { Franklin Island (Ross } \\
\text { Sea) }\end{array}$ & Utevsky (2005) \\
\hline 3100 & & & Nototheniobdella sawyeri & Parachaenichthys georgianus & Palmer Coast & Utevsky (2005) \\
\hline 3101 & & & Nototheniobdella sawyeri & Parachaenichthys georgianus & South Shetland Islands & Utevsky (2005) \\
\hline 3102 & & & Nototheniobdella sawyeri & Parachaenichthys georgianus & $\begin{array}{l}\text { Filchner Ice Shelf } \\
\text { (Weddell Sea) }\end{array}$ & Utevsky (2005) \\
\hline 3103 & & & Nototheniobdella sawyeri & Parachaenichthys georgianus & Clarie Coast & Utevsky (2005) \\
\hline 3104 & & & Nototheniobdella sawyeri & Parachaenichthys georgianus & Scott Coast & Utevsky (2005) \\
\hline 3105 & & & Nototheniobdella sawyeri & Parachaenichthys georgianus & $\begin{array}{l}\text { Franklin Island (Ross } \\
\text { Sea) }\end{array}$ & Utevsky (2005) \\
\hline
\end{tabular}




\begin{tabular}{|c|c|c|c|c|c|c|}
\hline Index & Phylum & Family & Species & Host & Location & References \\
\hline 3106 & & & Nototheniobdella sawyeri & Harpagiferidae & Palmer Coast & Utevsky (2005) \\
\hline 3107 & & & Nototheniobdella sawyeri & Harpagiferidae & South Shetland Islands & Utevsky (2005) \\
\hline 3108 & & & Nototheniobdella sawyeri & Harpagiferidae & $\begin{array}{l}\text { Filchner Ice Shelf } \\
\text { (Weddell Sea) }\end{array}$ & Utevsky (2005) \\
\hline 3109 & & & Nototheniobdella sawyeri & Harpagiferidae & Clarie Coast & Utevsky (2005) \\
\hline 3110 & & & Nototheniobdella sawyeri & Harpagiferidae & Scott Coast & Utevsky (2005) \\
\hline 3111 & & & Nototheniobdella sawyeri & Harpagiferidae & $\begin{array}{c}\text { Franklin Island (Ross } \\
\text { Sea) }\end{array}$ & Utevsky (2005) \\
\hline 3112 & \multirow{21}{*}{ ANNELIDA } & & Ophthalmobdella bellisioi & blank & blank & Szidat (1965) \\
\hline 3113 & & Piscicolidae & Ophthalmobdella bellisioi & Notothenia neglecta & Borge Bay (Signy Island) & Hoogesteger \& White (1981) \\
\hline 3114 & & & Trachelobdella bathyrajae & blank & blank & Meyer \& Burreson (1990) \\
\hline 3115 & & & Trachelobdella bathyrajae & Bathyraja maccaini & South Shetland Islands & Utevsky (2005) \\
\hline 3116 & & & Trulliobdella sp. & Notothenia neglecta & Borge Bay (Signy Island) & Hoogesteger \& White (1981) \\
\hline 3117 & & & Trulliobdella bacilliformis & blank & blank & Brinkmann, Jr. (1948) \\
\hline 3118 & & & Trulliobdella bacilliformis & Chaenichthys rhinoceratus & Bouvet Island & Utevsky (2005) \\
\hline 3119 & & & Trulliobdella bacilliformis & Chaenichthys rhinoceratus & South Shetland Islands & Utevsky (2005) \\
\hline 3120 & & & Trulliobdella bacilliformis & Chaenichthys rhinoceratus & South Orkney Islands & Utevsky (2005) \\
\hline 3121 & & & Trulliobdella bacilliformis & Chaenichthys rhinoceratus & South Georgia Island & Utevsky (2005) \\
\hline 3122 & & & Trulliobdella bacilliformis & Chaenichthys rhinoceratus & Prince Edward Island & Utevsky (2005) \\
\hline 3123 & & & Trulliobdella bacilliformis & Chaenichthys rhinoceratus & Marion Island & Utevsky (2005) \\
\hline 3124 & & & Trulliobdella bacilliformis & Chaenichthys rhinoceratus & Crozet Islands & Utevsky (2005) \\
\hline 3125 & & & Trulliobdella bacilliformis & Chaenichthys rhinoceratus & Kerguelen Subregion & Utevsky (2005) \\
\hline 3126 & & & Trulliobdella bacilliformis & Chaenocephalus aceratus & Bouvet Island & Utevsky (2005) \\
\hline 3127 & & & Trulliobdella bacilliformis & Chaenocephalus aceratus & South Shetland Islands & Utevsky (2005) \\
\hline 3128 & & & Trulliobdella bacilliformis & Chaenocephalus aceratus & South Orkney Islands & Utevsky (2005) \\
\hline 3129 & & & Trulliobdella bacilliformis & Chaenocephalus aceratus & South Georgia Island & Utevsky (2005) \\
\hline 3130 & & & Trulliobdella bacilliformis & Chaenocephalus aceratus & Prince Edward Island & Utevsky (2005) \\
\hline 3131 & & & Trulliobdella bacilliformis & Chaenocephalus aceratus & Marion Island & Utevsky (2005) \\
\hline 3132 & & & Trulliobdella bacilliformis & Chaenocephalus aceratus & Crozet Islands & Utevsky (2005) \\
\hline
\end{tabular}




\begin{tabular}{|c|c|c|c|c|c|c|}
\hline Index & Phylum & Family & Species & Host & Location & References \\
\hline 3133 & & & Trulliobdella bacilliformis & Chaenocephalus aceratus & Kerguelen Subregion & Utevsky (2005) \\
\hline 3134 & & & Trulliobdella bacilliformis & Chaenocephalus bouvetensis & Bouvet Island & Utevsky (2005) \\
\hline 3135 & & & Trulliobdella bacilliformis & Chaenocephalus bouvetensis & South Shetland Islands & Utevsky (2005) \\
\hline 3136 & & & Trulliobdella bacilliformis & Chaenocephalus bouvetensis & South Orkney Islands & Utevsky (2005) \\
\hline 3137 & & & Trulliobdella bacilliformis & Chaenocephalus bouvetensis & South Georgia Island & Utevsky (2005) \\
\hline 3138 & & & Trulliobdella bacilliformis & Chaenocephalus bouvetensis & Prince Edward Island & Utevsky (2005) \\
\hline 3139 & & & Trulliobdella bacilliformis & Chaenocephalus bouvetensis & Marion Island & Utevsky (2005) \\
\hline 3140 & & & Trulliobdella bacilliformis & Chaenocephalus bouvetensis & Crozet Islands & Utevsky (2005) \\
\hline 3141 & & & Trulliobdella bacilliformis & Chaenocephalus bouvetensis & Kerguelen Subregion & Utevsky (2005) \\
\hline 3142 & \multirow{18}{*}{ ANNELIDA } & Piscicolidae & Trulliobdella bacilliformis & Champsocephalus gunnari & Bouvet Island & Utevsky (2005) \\
\hline 3143 & & & Trulliobdella bacilliformis & Champsocephalus gunnari & South Shetland Islands & Utevsky (2005) \\
\hline 3144 & & & Trulliobdella bacilliformis & Champsocephalus gunnari & South Orkney Islands & Utevsky (2005) \\
\hline 3145 & & & Trulliobdella bacilliformis & Champsocephalus gunnari & South Georgia Island & Utevsky (2005) \\
\hline 3146 & & & Trulliobdella bacilliformis & Champsocephalus gunnari & Prince Edward Island & Utevsky (2005) \\
\hline 3147 & & & Trulliobdella bacilliformis & Champsocephalus gunnari & Marion Island & Utevsky (2005) \\
\hline 3148 & & & Trulliobdella bacilliformis & Champsocephalus gunnari & Crozet Islands & Utevsky (2005) \\
\hline 3149 & & & Trulliobdella bacilliformis & Champsocephalus gunnari & Kerguelen Subregion & Utevsky (2005) \\
\hline 3150 & & & Trulliobdella bacilliformis & Champsocephalus rastrospinosus & Bouvet Island & Utevsky (2005) \\
\hline 3151 & & & Trulliobdella bacilliformis & Champsocephalus rastrospinosus & South Shetland Islands & Utevsky (2005) \\
\hline 3152 & & & Trulliobdella bacilliformis & Champsocephalus rastrospinosus & South Orkney Islands & Utevsky (2005) \\
\hline 3153 & & & Trulliobdella bacilliformis & Champsocephalus rastrospinosus & South Georgia Island & Utevsky (2005) \\
\hline 3154 & & & Trulliobdella bacilliformis & Champsocephalus rastrospinosus & Prince Edward Island & Utevsky (2005) \\
\hline 3155 & & & Trulliobdella bacilliformis & Champsocephalus rastrospinosus & Marion Island & Utevsky (2005) \\
\hline 3156 & & & Trulliobdella bacilliformis & Champsocephalus rastrospinosus & Crozet Islands & Utevsky (2005) \\
\hline 3157 & & & Trulliobdella bacilliformis & Champsocephalus rastrospinosus & Kerguelen Subregion & Utevsky (2005) \\
\hline 3158 & & & Trulliobdella bacilliformis & Notothenia coriiceps & Bouvet Island & Utevsky (2005) \\
\hline 3159 & & & Trulliobdella bacilliformis & Notothenia coriiceps & South Shetland Islands & Utevsky (2005) \\
\hline
\end{tabular}




\begin{tabular}{|c|c|c|c|c|c|c|}
\hline Index & Phylum & Family & Species & Host & Location & References \\
\hline 3160 & & & Trulliobdella bacilliformis & Notothenia coriiceps & South Orkney Islands & Utevsky (2005) \\
\hline 3161 & & & Trulliobdella bacilliformis & Notothenia coriiceps & South Georgia Island & Utevsky (2005) \\
\hline 3162 & & & Trulliobdella bacilliformis & Notothenia coriiceps & Prince Edward Island & Utevsky (2005) \\
\hline 3163 & & & Trulliobdella bacilliformis & Notothenia coriiceps & Marion Island & Utevsky (2005) \\
\hline 3164 & & & Trulliobdella bacilliformis & Notothenia coriiceps & Crozet Islands & Utevsky (2005) \\
\hline 3165 & & & Trulliobdella bacilliformis & Notothenia coriiceps & Kerguelen Subregion & Utevsky (2005) \\
\hline 3166 & & & Trulliobdella bacilliformis & Notothenia neglecta & Bouvet Island & Utevsky (2005) \\
\hline 3167 & & & Trulliobdella bacilliformis & Notothenia neglecta & South Shetland Islands & Utevsky (2005) \\
\hline 3168 & & & Trulliobdella bacilliformis & Notothenia neglecta & South Orkney Islands & Utevsky (2005) \\
\hline 3169 & & & Trulliobdella bacilliformis & Notothenia neglecta & South Georgia Island & Utevsky (2005) \\
\hline 3170 & & & Trulliobdella bacilliformis & Notothenia neglecta & Prince Edward Island & Utevsky (2005) \\
\hline 3171 & \multirow{16}{*}{ ANNELIDA } & Piscicolidae & Trulliobdella bacilliformis & Notothenia neglecta & Marion Island & Utevsky (2005) \\
\hline 3172 & & & Trulliobdella bacilliformis & Notothenia neglecta & Crozet Islands & Utevsky (2005) \\
\hline 3173 & & & Trulliobdella bacilliformis & Notothenia neglecta & Kerguelen Subregion & Utevsky (2005) \\
\hline 3174 & & & Trulliobdella bacilliformis & Nototheniops larseni & Bouvet Island & Utevsky (2005) \\
\hline 3175 & & & Trulliobdella bacilliformis & Nototheniops larseni & South Shetland Islands & Utevsky (2005) \\
\hline 3176 & & & Trulliobdella bacilliformis & Nototheniops larseni & South Orkney Islands & Utevsky (2005) \\
\hline 3177 & & & Trulliobdella bacilliformis & Nototheniops larseni & South Georgia Island & Utevsky (2005) \\
\hline 3178 & & & Trulliobdella bacilliformis & Nototheniops larseni & Prince Edward Island & Utevsky (2005) \\
\hline 3179 & & & Trulliobdella bacilliformis & Nototheniops larseni & Marion Island & Utevsky (2005) \\
\hline 3180 & & & Trulliobdella bacilliformis & Nototheniops larseni & Crozet Islands & Utevsky (2005) \\
\hline 3181 & & & Trulliobdella bacilliformis & Nototheniops larseni & Kerguelen Subregion & Utevsky (2005) \\
\hline 3182 & & & Trulliobdella bacilliformis & Parachaenichthys georgianus & Bouvet Island & Utevsky (2005) \\
\hline 3183 & & & Trulliobdella bacilliformis & Parachaenichthys georgianus & South Shetland Islands & Utevsky (2005) \\
\hline 3184 & & & Trulliobdella bacilliformis & Parachaenichthys georgianus & South Orkney Islands & Utevsky (2005) \\
\hline 3185 & & & Trulliobdella bacilliformis & Parachaenichthys georgianus & South Georgia Island & Utevsky (2005) \\
\hline 3186 & & & Trulliobdella bacilliformis & Parachaenichthys georgianus & Prince Edward Island & Utevsky (2005) \\
\hline
\end{tabular}




\begin{tabular}{|c|c|c|c|c|c|c|}
\hline Index & Phylum & Family & Species & Host & Location & References \\
\hline 3187 & & & Trulliobdella bacilliformis & Parachaenichthys georgianus & Marion Island & Utevsky (2005) \\
\hline 3188 & & & Trulliobdella bacilliformis & Parachaenichthys georgianus & Crozet Islands & Utevsky (2005) \\
\hline 3189 & & & Trulliobdella bacilliformis & Parachaenichthys georgianus & Kerguelen Subregion & Utevsky (2005) \\
\hline 3190 & & & Trulliobdella bacilliformis & Pseudochaenichthys georgianus & Bouvet Island & Utevsky (2005) \\
\hline 3191 & & & Trulliobdella bacilliformis & Pseudochaenichthys georgianus & South Shetland Islands & Utevsky (2005) \\
\hline 3192 & & & Trulliobdella bacilliformis & Pseudochaenichthys georgianus & South Orkney Islands & Utevsky (2005) \\
\hline 3193 & & & Trulliobdella bacilliformis & Pseudochaenichthys georgianus & South Georgia Island & Utevsky (2005) \\
\hline 3194 & & & Trulliobdella bacilliformis & Pseudochaenichthys georgianus & Prince Edward Island & Utevsky (2005) \\
\hline 3195 & & & Trulliobdella bacilliformis & Pseudochaenichthys georgianus & Marion Island & Utevsky (2005) \\
\hline 3196 & & & Trulliobdella bacilliformis & Pseudochaenichthys georgianus & Crozet Islands & Utevsky (2005) \\
\hline 3197 & & & Trulliobdella bacilliformis & Pseudochaenichthys georgianus & Kerguelen Subregion & Utevsky (2005) \\
\hline 3198 & & & Trulliobdella capitis & blank & blank & Brinkmann (1947) \\
\hline 3199 & & & Trulliobdella capitis & Chaenichthys rhinoceratus & Bouvet Island & Utevsky (2005) \\
\hline 3200 & \multirow{14}{*}{ ANNELIDA } & Piscicolidae & Trulliobdella capitis & Chaenichthys rhinoceratus & South Shetland Islands & Utevsky (2005) \\
\hline 3201 & & & Trulliobdella capitis & Chaenichthys rhinoceratus & South Orkney Islands & Utevsky (2005) \\
\hline 3202 & & & Trulliobdella capitis & Chaenichthys rhinoceratus & Weddell Sea & Utevsky (2005) \\
\hline 3203 & & & Trulliobdella capitis & Chaenichthys rhinoceratus & Wilkes Land & Utevsky (2005) \\
\hline 3204 & & & Trulliobdella capitis & Chaenichthys rhinoceratus & Argentine Island & Utevsky (2005) \\
\hline 3205 & & & Trulliobdella capitis & Chaenichthys rhinoceratus & Ross Sea & Utevsky (2005) \\
\hline 3206 & & & Trulliobdella capitis & Chaenocephalus aceratus & South Georgia Island & Siegel (1980b) \\
\hline 3207 & & & Trulliobdella capitis & Chaenocephalus aceratus & South Orkney Islands & Siegel (1980b) \\
\hline 3208 & & & Trulliobdella capitis & Chaenocephalus aceratus & South Shetland Islands & Siegel (1980b) \\
\hline 3209 & & & Trulliobdella capitis & Chaenocephalus bouvetensis & Bouvet Island & Utevsky (2005) \\
\hline 3210 & & & Trulliobdella capitis & Chaenocephalus bouvetensis & South Shetland Islands & Utevsky (2005) \\
\hline 3211 & & & Trulliobdella capitis & Chaenocephalus bouvetensis & South Orkney Islands & Utevsky (2005) \\
\hline 3212 & & & Trulliobdella capitis & Chaenocephalus bouvetensis & Weddell Sea & Utevsky (2005) \\
\hline 3213 & & & Trulliobdella capitis & Chaenocephalus bouvetensis & Wilkes Land & Utevsky (2005) \\
\hline
\end{tabular}




\begin{tabular}{|c|c|c|c|c|c|c|}
\hline Index & Phylum & Family & Species & Host & Location & References \\
\hline 3214 & & & Trulliobdella capitis & Chaenocephalus bouvetensis & Argentine Island & Utevsky (2005) \\
\hline 3215 & & & Trulliobdella capitis & Chaenocephalus bouvetensis & Ross Sea & Utevsky (2005) \\
\hline 3216 & & & Trulliobdella capitis & Chaenodraco wilsoni & Weddell Sea & Kock et al. (1984) \\
\hline 3217 & & & Trulliobdella capitis & Chaenodraco wilsoni & Bouvet Island & Utevsky (2005) \\
\hline 3218 & & & Trulliobdella capitis & Chaenodraco wilsoni & South Shetland Islands & Utevsky (2005) \\
\hline 3219 & & & Trulliobdella capitis & Chaenodraco wilsoni & South Orkney Islands & Utevsky (2005) \\
\hline 3220 & & & Trulliobdella capitis & Chaenodraco wilsoni & Weddell Sea & Utevsky (2005) \\
\hline 3221 & & & Trulliobdella capitis & Chaenodraco wilsoni & Wilkes Land & Utevsky (2005) \\
\hline 3222 & & & Trulliobdella capitis & Chaenodraco wilsoni & Argentine Island & Utevsky (2005) \\
\hline 3223 & & & Trulliobdella capitis & Chaenodraco wilsoni & Ross Sea & Utevsky (2005) \\
\hline 3224 & & & Trulliobdella capitis & Champsocephalus gunnari & South Georgia Island & Siegel (1980b) \\
\hline 3225 & & & Trulliobdella capitis & Champsocephalus gunnari & Bouvet Island & Utevsky (2005) \\
\hline 3226 & & & Trulliobdella capitis & Champsocephalus gunnari & South Shetland Islands & Utevsky (2005) \\
\hline 3227 & & & Trulliobdella capitis & Champsocephalus gunnari & South Orkney Islands & Utevsky (2005) \\
\hline 3228 & & & Trulliobdella capitis & Champsocephalus gunnari & Weddell Sea & Utevsky (2005) \\
\hline 3229 & \multirow{12}{*}{ ANNELIDA } & Piscicolidae & Trulliobdella capitis & Champsocephalus gunnari & Wilkes Land & Utevsky (2005) \\
\hline 3230 & & & Trulliobdella capitis & Champsocephalus gunnari & Argentine Island & Utevsky (2005) \\
\hline 3231 & & & Trulliobdella capitis & Champsocephalus gunnari & Ross Sea & Utevsky (2005) \\
\hline 3232 & & & Trulliobdella capitis & Chiondraco rastrospinosus & Bouvet Island & Utevsky (2005) \\
\hline 3233 & & & Trulliobdella capitis & Chiondraco rastrospinosus & South Shetland Islands & Utevsky (2005) \\
\hline 3234 & & & Trulliobdella capitis & Chiondraco rastrospinosus & South Orkney Islands & Utevsky (2005) \\
\hline 3235 & & & Trulliobdella capitis & Chiondraco rastrospinosus & Weddell Sea & Utevsky (2005) \\
\hline 3236 & & & Trulliobdella capitis & Chiondraco rastrospinosus & Wilkes Land & Utevsky (2005) \\
\hline 3237 & & & Trulliobdella capitis & Chiondraco rastrospinosus & Argentine Island & Utevsky (2005) \\
\hline 3238 & & & Trulliobdella capitis & Chiondraco rastrospinosus & Ross Sea & Utevsky (2005) \\
\hline 3239 & & & Trulliobdella capitis & Chionodraco hamatus & Bouvet Island & Utevsky (2005) \\
\hline 3240 & & & Trulliobdella capitis & Chionodraco hamatus & South Shetland Islands & Utevsky (2005) \\
\hline
\end{tabular}




\begin{tabular}{|c|c|c|c|c|c|c|}
\hline Index & Phylum & Family & Species & Host & Location & References \\
\hline 3241 & & & Trulliobdella capitis & Chionodraco hamatus & South Orkney Islands & Utevsky (2005) \\
\hline 3242 & & & Trulliobdella capitis & Chionodraco hamatus & Weddell Sea & Utevsky (2005) \\
\hline 3243 & & & Trulliobdella capitis & Chionodraco hamatus & Wilkes Land & Utevsky (2005) \\
\hline 3244 & & & Trulliobdella capitis & Chionodraco hamatus & Argentine Island & Utevsky (2005) \\
\hline 3245 & & & Trulliobdella capitis & Chionodraco hamatus & Ross Sea & Utevsky (2005) \\
\hline 3246 & & & Trulliobdella capitis & Chionodraco kathleenae & Bouvet Island & Utevsky (2005) \\
\hline 3247 & & & Trulliobdella capitis & Chionodraco kathleenae & South Shetland Islands & Utevsky (2005) \\
\hline 3248 & & & Trulliobdella capitis & Chionodraco kathleenae & South Orkney Islands & Utevsky (2005) \\
\hline 3249 & & & Trulliobdella capitis & Chionodraco kathleenae & Weddell Sea & Utevsky (2005) \\
\hline 3250 & & & Trulliobdella capitis & Chionodraco kathleenae & Wilkes Land & Utevsky (2005) \\
\hline 3251 & & & Trulliobdella capitis & Chionodraco kathleenae & Argentine Island & Utevsky (2005) \\
\hline 3252 & & & Trulliobdella capitis & Chionodraco kathleenae & Ross Sea & Utevsky (2005) \\
\hline 3253 & & & Trulliobdella capitis & Chionodraco sp. & South Orkney Islands & Siegel (1980b) \\
\hline 3254 & & & Trulliobdella capitis & Cryodraco antarcticus & Bouvet Island & Utevsky (2005) \\
\hline 3255 & & & Trulliobdella capitis & Cryodraco antarcticus & South Shetland Islands & Utevsky (2005) \\
\hline 3256 & & & Trulliobdella capitis & Cryodraco antarcticus & South Orkney Islands & Utevsky (2005) \\
\hline 3257 & & & Trulliobdella capitis & Cryodraco antarcticus & Weddell Sea & Utevsky (2005) \\
\hline 3258 & \multirow{10}{*}{ ANNELIDA } & Piscicolidae & Trulliobdella capitis & Cryodraco antarcticus & Wilkes Land & Utevsky (2005) \\
\hline 3259 & & & Trulliobdella capitis & Cryodraco antarcticus & Argentine Island & Utevsky (2005) \\
\hline 3260 & & & Trulliobdella capitis & Cryodraco antarcticus & Ross Sea & Utevsky (2005) \\
\hline 3261 & & & Trulliobdella capitis & Neopagetopsis ionah & Bouvet Island & Utevsky (2005) \\
\hline 3262 & & & Trulliobdella capitis & Neopagetopsis ionah & South Shetland Islands & Utevsky (2005) \\
\hline 3263 & & & Trulliobdella capitis & Neopagetopsis ionah & South Orkney Islands & Utevsky (2005) \\
\hline 3264 & & & Trulliobdella capitis & Neopagetopsis ionah & Weddell Sea & Utevsky (2005) \\
\hline 3265 & & & Trulliobdella capitis & Neopagetopsis ionah & Wilkes Land & Utevsky (2005) \\
\hline 3266 & & & Trulliobdella capitis & Neopagetopsis ionah & Argentine Island & Utevsky (2005) \\
\hline 3267 & & & Trulliobdella capitis & Neopagetopsis ionah & Ross Sea & Utevsky (2005) \\
\hline
\end{tabular}




\begin{tabular}{|c|c|c|c|c|c|c|}
\hline Index & Phylum & Family & Species & Host & Location & References \\
\hline 3268 & & & Trulliobdella capitis & Notothenia coriiceps & Bouvet Island & Utevsky (2005) \\
\hline 3269 & & & Trulliobdella capitis & Notothenia coriiceps & South Shetland Islands & Utevsky (2005) \\
\hline 3270 & & & Trulliobdella capitis & Notothenia coriiceps & South Orkney Islands & Utevsky (2005) \\
\hline 3271 & & & Trulliobdella capitis & Notothenia coriiceps & Weddell Sea & Utevsky (2005) \\
\hline 3272 & & & Trulliobdella capitis & Notothenia coriiceps & Wilkes Land & Utevsky (2005) \\
\hline 3273 & & & Trulliobdella capitis & Notothenia coriiceps & Argentine Island & Utevsky (2005) \\
\hline 3274 & & & Trulliobdella capitis & Notothenia coriiceps & Ross Sea & Utevsky (2005) \\
\hline 3275 & & & Trulliobdella capitis & Notothenia rossii & Bouvet Island & Utevsky (2005) \\
\hline 3276 & & & Trulliobdella capitis & Notothenia rossii & South Shetland Islands & Utevsky (2005) \\
\hline 3277 & & & Trulliobdella capitis & Notothenia rossii & South Orkney Islands & Utevsky (2005) \\
\hline 3278 & & & Trulliobdella capitis & Notothenia rossii & Weddell Sea & Utevsky (2005) \\
\hline 3279 & & & Trulliobdella capitis & Notothenia rossii & Wilkes Land & Utevsky (2005) \\
\hline 3280 & & & Trulliobdella capitis & Notothenia rossii & Argentine Island & Utevsky (2005) \\
\hline 3281 & & & Trulliobdella capitis & Notothenia rossii & Ross Sea & Utevsky (2005) \\
\hline 3282 & & & Trulliobdella capitis & Parachaenichthys georgianus & Bouvet Island & Utevsky (2005) \\
\hline 3283 & & & Trulliobdella capitis & Parachaenichthys georgianus & South Shetland Islands & Utevsky (2005) \\
\hline 3284 & & & Trulliobdella capitis & Parachaenichthys georgianus & South Orkney Islands & Utevsky (2005) \\
\hline 3285 & & & Trulliobdella capitis & Parachaenichthys georgianus & Weddell Sea & Utevsky (2005) \\
\hline 3286 & & & Trulliobdella capitis & Parachaenichthys georgianus & Wilkes Land & Utevsky (2005) \\
\hline 3287 & \multirow{8}{*}{ ANNELIDA } & Piscicolidae & Trulliobdella capitis & Parachaenichthys georgianus & Argentine Island & Utevsky (2005) \\
\hline 3288 & & & Trulliobdella capitis & Parachaenichthys georgianus & Ross Sea & Utevsky (2005) \\
\hline 3289 & & & Trulliobdella capitis & Pseudochaenichthys georgianus & South Georgia Island & Siegel (1980b) \\
\hline 3290 & & & Trulliobdella capitis & Pseudochaenichthys georgianus & South Orkney Islands & Siegel (1980b) \\
\hline 3291 & & & Trulliobdella capitis & Pseudochaenichthys georgianus & Bouvet Island & Utevsky (2005) \\
\hline 3292 & & & Trulliobdella capitis & Pseudochaenichthys georgianus & South Shetland Islands & Utevsky (2005) \\
\hline 3293 & & & Trulliobdella capitis & Pseudochaenichthys georgianus & South Orkney Islands & Utevsky (2005) \\
\hline 3294 & & & Trulliobdella capitis & Pseudochaenichthys georgianus & Weddell Sea & Utevsky (2005) \\
\hline
\end{tabular}




\begin{tabular}{|c|c|c|c|c|c|c|}
\hline Index & Phylum & Family & Species & Host & Location & References \\
\hline 3295 & & & Trulliobdella capitis & Pseudochaenichthys georgianus & Wilkes Land & Utevsky (2005) \\
\hline 3296 & & & Trulliobdella capitis & Pseudochaenichthys georgianus & Argentine Island & Utevsky (2005) \\
\hline 3297 & & & Trulliobdella capitis & Pseudochaenichthys georgianus & Ross Sea & Utevsky (2005) \\
\hline
\end{tabular}


Peer-review: Externally peer-reviewed

Author Contributions: Concept - M.C.O.; Design - M.C.O., M.C.B., Y.T.; Supervision - M.C.B., M.C.O.; Funding - M.C.O., R.A.B.; Materials - M.C.O., R.A.B.; Data Collection and/or Processing - M.C.O., Y.T., B.A.; Analysis and/ or Interpretation - M.C.B., R.A.H.; Literature Review - M.C.O., M.C.B., R.A.B.; Writer - M.C.B., Y.T., B.A., M.G., Ü.A.; Critical Review - M.C.B., M.C.O.

Conflict of Interest: No conflict of interest was declared by the authors.

Financial Disclosure: The authors declared that this study has received no financial support.

Hakem Değerlendirmesi: Dış Bağımsız.

Yazar Katkıları: Fikir - M.C.O.; Tasarım - M.C.O., M.C.B., Y.T.; Denetleme - M.C.B., M.C.O.; Kaynaklar - M.C.O., R.A.B.; Malzemeler - M.C.O., R.A.B.; Veri Toplanması ve/veya işlemesi - M.C.O., Y.T., B.A.; Analiz ve/veya Yorum - M.C.B., R.A.H.; Literatür taraması - M.C.O., M.C.B., R.A.B.; Yazıyı Yazan M.C.B., Y.T., B.A., M.G., Ü.A.; Eleştirel İnceleme - M.C.B., M.C.O.

Çıkar Çatışması: Yazarlar çıkar çatışması bildirmemişlerdir.

Finansal Destek: Yazarlar bu çalışma için finansal destek almadıklarını beyan etmişlerdir.

\section{REFERENCES}

1. Bray RA, Zdzitowiecki, K. Boreascotia megavesicula n. g., n. sp. (Digenea: Hemiuridae: Lecithochiriinae) in the nototheniid fish Lepidonotothen macrophthalma (Norman) from the sub-Antarctic Atlantic. Syst Parasitol 2000; 46: 29-32. [CrossRef]

2. Brickle P, MacKenzie K, Pike A. Parasites of the Patagonian toothfish, Dissostichus eleginoides, in different parts of the Subantarctic. Polar Biol 2005; 28: 663-71. [CrossRef]

3. Brickle P, MacKenzie K, Pike A. Variations in the parasite fauna of the Patagonian toothfish (Dissostichus eleginoides Smitt, 1898), with length, season and depth of habitat around the Falkland Islands. J Parasitol 2006; 92: 282-91. [CrossRef]

4. Dzido J, Kijewska A, Rokicka M, Swiatalska-Koseda A, Rokicki J. Report on anisakid nematodes in polar regions e? Preliminary results. Polar Sci 2009; 3: 207-11. [CrossRef]

5. Froese R, Pauly D, editors. FishBase. (serial online) 2012 (cited 2013 March 6). Available from: http://www.fishbase.org/.

6. Gaevskaya AV, Rodyuk GN. Ecological characteristics of the parasite fauna of Macrourus carinatus Gunther. Biologicheskie Nauki 1988; 2: 21-5. [In Russian]

7. Gaevskaya AV, Rodyuk GN. Ecological characteristics of the parasite fauna of Macrourus carinatus Gunther. Biologicheskie Nauki 1988; 2:21-5. [In Russian]

8. Gaevskaya AV, Kovaljova AA, Parukhin AM. Peculiarities and formation of parasitofauna of the Patagonian toothfish Dissostichus eleginoides. Biologiya Morya 1990; 4: 23-8. [In Russian]

9. Gaevskaya AV, Kovaleva AA. Materials on fish trematodes in the south-western Atlantic. Vestnik Zoologii 1978; 3: 60-6. [In Russian]

10. Hargis WJJr, Dillon WA. Helminth Parasites of Antarctic Vertebrates. Part IV. Monogenetic Trematodes from Antarctic Fishes: The Superfamily Capsaloidea Price, 1936. Proc Biol Soc of Washington 1968; 81: 403-12

11. Holloway HLJr, Klewer HL. Rhabdochona beatriceinsleyae n. sp. (Nematoda: Spiruridea: Rhabdochonidae), from the Antarctic Zoarcid, Rhigophila dearborni. Trans Am Microscopical Soc 1969; 88: 460-71. [CrossRef]

12. Hoogesteger JN, White MG. Notes on Parasite Infestation of Inshore Fish at Signy Island, South Orkney Islands. Br Antarctic Surv Bull 1981; 54: 23-31.

13. Jezewski W, Laskowski Z, Zdzitowiecki K. Description of a new digenean (Zoogonidae) genus and species from Sub-Antarctic fish
Patagonotothen tessellata. J Parasitol 2009; 95: 1489-92. [CrossRef]

14. Johnston TH. New trematodes from subantarctic and Antarctic Aust J ExpBiol and Med Sci 1931; 8: 91-8. [CrossRef]

15. Klimpel S, Busch MW, Kuhn T, Rohde A, Palm HW. The Anisakis simplex complex off the South Shetland Islands (Antarctica) endemic populations versus introduction through migratory hosts. Mar Ecology Prog Series 2010; 403: 1-11. [CrossRef]

16. Kloser H, Plotz J, Palm H, Bartsch A, Hubold G. Adjustment of anisakid nematode life cycles to the high Antarctic food web as shown by Contracaecum radiatum and C. osculatum in the Weddell Sea. Antarctic Sci 1992; 4: 171-8. [CrossRef]

17. Kock KH, Schneppenheim R, Siegel V. A contribution to the fish fauna of the Weddell sea. Archiv fur Fischerei Wissenschaft 1984; 34: 103-20.

18. Kovalyova AA, Gaevskaya AV. Two species of Monogenea parasites of Antarctic fishes. Zoologiceskij J 1977; 56: 783-6.

19. Laskowski Z, Zdzitowiecki K. New morphological data on a sub-Antarctic acanthocephalan, Aspersentisjohni (Baylis, 1929) (Palaeacanthocephala: Heteracanthocephalidae). Syst Parasitol 2004; 59: 39-44. [CrossRef]

20. Laskowski Z, Zdzitowiecki K. The Helminth Fauna of Some Notothenioid Fishes Collected from the Shelf of Argentine Islands, West Antarctica. Pol Polar Res 2005; 26: 315-24.

21. Laskowski Z, Zdzitowiecki K. New morphological data on theacanthocephalan Hypoechinorhynchus magellanicus Szidat, 1950 (Palaeacanthocephala: Arhythmacanthidae). Syst Parasitolol 2008; 69: 179-83. [CrossRef]

22. Laskowski Z, Zdzitowiecki K. Occurrence of Acanthocephalans in Notothenioid Fishes in the Beagle Channel (Magellanic SubRegion, Sub-Antarctic). Pol Polar Res 2009; 30: 179-86.

23. Laskowski Z, Rocka A, Zdzitowiecki K, Ghigliotti L, Pisano E. New data on the occurrence of internal parasitic worms in the Gymnodraco acuticeps and Cygnodraco mawsoni (Bathydraconidae) fish in the Ross Sea, Antarctica. Pol Polar Res 2005; 26: 37-40.

24. Laskowski Z, Rocka A, Zdzitowiecki K, Ozouf-Costaz C. Occurrence of endoparasitic worms in dusky notothen, Trematomus newnesi (Actinopterygii Nototheniidae), at Adélie Land, Antarctica. Pol Polar Res 2007; 28: 37-42.

25. Laskowski Z, Jezewski W, Zdzitowiecki K. Cystacanths of Acanthocephala in notothenioid fish from the Beagle Channel (sub-Antarctica). Syst Parasitol 2008; 70: 107-17. [CrossRef]

26. Lutnicka $\mathrm{H}$, Zdzitowiecki K. On Some Problems related to the occurrence of Pseudobenedenia nototheniae Johnston, 1931 (Monogenea, Capsalidae, Trochopodinae) off the South Shetland Islands. Acta Ichthyologica et Piscatoria 1984; 14: 141-7.

27. Lyadov VN. Zoogeographical characteristics of the Helminths of Fishes from the Antarctic Zone of the World Ocean. NOAA Technical Rep NMFS 1985; 25: 41-3.

28. Lyadov VN, Parukhin AM, Mironova AV. Helminth Fauna of the Family Chaenichthidae from the Region of Kerguelen Subregion Islands. Zoologicheskii Zhurnal 1981; 60: 142-4.

29. Moser M, Cowen RK. The Effects of Periodic Eutrophication on Parasitism and Stock Identification of Trematomus bernacchii (Pisces: Nototheniidae) in McMurdo Sound. The J Parasitol 1991; 77: 551-6. [CrossRef]

30. Palm HW. Ecology of Pseudoterranova decipiens (Krabbe, 1878) (Nematoda: Anisakidae) from Antarctic Waters. Parasitological Res 1999; 85: 638-46. [CrossRef]

31. Palm HW, Reimann N, Splinder M, Plötz J. The Role of the Rock Cod Notothenia coriiceps in the Life-cycle of Antarctic Parasites. Polar Biol 1998; 19: 399-406. [CrossRef]

32. Palm HW, Klimpe S, Walter T. Demersal Fish Parasite Fauna Around the South Shetland Islands; High Species Richness and Low Host Specificity in Deep Antarctic Waters. Polar Biol 2007; 30: 1513-22. [CrossRef] 
33. Parukhin AM. Helminthofauna peculiarities of commercial Nototheniodei from the Subantarktic region of the Indian Ocean. Vestnik Zoologii 1986; 3: 6-10.

34. Parukhin AM, Lyadov VN. Parasitofauna of Notothenioidei from waters of the Atlantic and Indian Oceans. Vestnik Zoologii 1981; 3: 90-4. [in Russian]

35. Parukhin AM, Lyadov VN. Helminth fauna of food Nototheniidae fishes from Kerguelen Subregion region. Ekologiya Morya. 1982; 10:49-56. [in Russian]

36. Prudhoe S, Bray RA. Digenetic trematodes from fishes. B.A.N.Z. Antarctic Res Expedition, Reports B (Zoology and Botany) 1973; 8: 195-225.

37. Reimer LW, Jessen O. Ein Beitrag zur Parasitenfauna von Merluccius hubbsi Marini. Wiss. Z. Pad. Hochsch. "Liseloune-Hermann". Gustrow 1974; 2: 53-64.

38. Rocka A. Biometrical variability and occurrence of Ascarophis nototheniae (Nematoda, Cystidicolidae), a parasitic nematode of Antarctic and subantarctic fishes. Acta Parasitologica 1999; 44: 188-192.

39. Rocka A. Cestodes of the Antarctic Fishes. Pol Polar Res 2003; 24: 261-76.

40. Rocka A. Helminths of Antarctic fishes: Life cycle biology, specificity and geographical distribution. Acta Parasitolologica 2006; 51: 26-35. [CrossRef]

41. Rocka A, Zdzitowiecki K. Cestodes in Fishes of the Weddell Sea. Acta Parasitologica 1998; 43: 64-70.

42. Rohde K, Hayward C, Heap M. Aspects of the ecology of metazoan ectoparasites of marine fishes. Int J Parasitol 1995; 25: 945-70. [CrossRef]

43. Rohde K, Ho J, Smales L, and Williams R. Parasites of Antarctic Fishes: Monogenea, Copepoda and Acanthocephala. Mar Freshwater Res 1998; 49: 121-5. [CrossRef]

44. Rokicka M. Report on Species of Gyrodactylus Nordman, 1832. Distribution in Polar Regions. Polar Sci 2009; 3: 203-6. [CrossRef]

45. Rokicka M, Lumme J, Zietara MS. Two New Antarctic Gyrodactylus Species (Monogenoidea): Description and Phylogenetic Characterization. The J Parasitol 2009; 95: 1112-9. [CrossRef]

46. Rokicki J, Rodjuk G, Zdzitowiecki K. Larval ascaridoid nematodes (Anisakidae) in fish from the South Shetland Islands (Southern Ocean). Pol Polar Res 2009; 30: 49-58.

47. Ruhl HA, Hastings PA, Zarubick LA, Jensen RM, Zdzitowiecki K. Fish populations of Port Foster, Deception Island, Antarctica and vicinity. Deep-Sea Res II 2003; 50: 1843-58. [CrossRef]

48. Sawyer RT. A new species of "tentacled" marine fish leech parasitic on Notothenia from the Subantarctic Marion and Crozet Islands. Hydrobiologia 1972; 40: 345-54. [CrossRef]

49. Sawyer RT, Villiers AF. Notes on two marine leeches (Annelida: Hirudinea) from Subantarctic Marion Island including a new record. Polar Hydrobiologia 1976; 48: 267-8. [CrossRef]

50. Siegel V. Parasite tags for some Antarctic Channicthyid fish. Archiv fur 1Fischerei Wissenschaft 1980; 31: 97-103.

51. Siegel V. Quantitative investigations on parasites of Antarctic Channichthyid and Nototheniid fishes. Meeresforschung 1980; 28 : 146-256.

52. Szidat L. Estudios sobre la fauna de Parasitos de peces Antarticos I. Los parasitos de Notothenia neglecta Nybelin. Servicio de Hidrografia Naval, Secretaria de Marina, República Argentina, Público H, 910, Buenos Aires: 1965; 1-57.

53. Szidat L. Estudios sobre la fauna de Parasitos de peces Antarticos III. Pseudobenedenoides branchicola gen.nov.sp.nov. (Trematoda, Monogenea, Capsalidae) un Nuevo parasite branquial del pez Antartico "Trematomus bernacchii". De Neotropica 1969; 15: 25-32.

54. Szidat L, Graefe G. Estudios sobre la fauna de parasitos de peces antarticos II-Los parasitos de Parachaenichthys charcoti. Servicio de
Hidrografia Naval, Armada Argentina,República Argentina, Público H, 911, Buenos Aires: 1967; 1-27.

55. Utevsky A Yu. An identification key to Antarctic fish leeches (Hirudinea:Piscicolidae). Ukrainian Antarctic J 2005; 3: 135-44.

56. Whittington ID. Diversity "down under" monogeneans in the Antipodes (Australia) with a prediction of monogenean biodiversity worldwide. Int J for Parasitol 1998; 28: 1481-93. [CrossRef]

57. Wojciechowska A. Pseudanthobothrium shetlandicum sp.n. and P. notogeorgianum sp.n. (Tetraphyllidea) from rays in the regions of the South Shetland Islands and South Georgia (Antarctic). Acta Parasitologica Polonica 1990; 35: 181-6.

58. Wojciechowska A. The tetraphyllidean and tetrabothriid cercoids from Antarctic bony fishes. I. Morphology. Identification with adult forms. Acta Parasitologica 1993; 38: 15-22.

59. Wojciechowska A. The tetraphyllidean and tetrabothriid cercoids from Antarctic bony fishes. II. Occurrence of cercoids in various fish species. Acta Parasitologica 1993; 38: 113-8.

60. Wojciechowska A, Pisano E, Zdzitowiecki K. Cestodes in fishes at the Heard Island (Subatarctic). Pol Polar Res 1995; 16: 205-12.

61. Wojciechowska A, Zdzitowiecki K, Pisano E, Vacchi M. The tetraphyllidean cercoids from bony fishes occurring in the Ross Sea (Antarctic). Acta Parasitologica 1994; 39: 13-5.

62. Zdzitoweicki K. Echinorhyncus nototheniae sp.n. (Acanthocephala) from nototheniid fishes from the environs of South Shetland Islands (Antarctic). Acta Parasitologica Polonica 1986; 31: 23-7.

63. Zdzitoweicki K. Acanthocephalans of marine fishes in the regions of South Georgia and South Orkneys (Antarctic). Acta Parasitologica Polonica 1987; 31: 211-7.

64. Zdzitowiecki K. Digenetic trematodes in alimentary tracts of fishes of south Georgia ans South Shetland Islands (Antarctica). Acta Ichthyologica et Piscatoria 1979; 9: 15-30.

65. Zdzitowiecki K. Prevalence of acanthocephalans in fishes of South Shetland Islands (Antactic) I. Juvenile Corynosoma spp. Acta Parasiitologica Polonica 1986; 30: 143-71.

66. Zdzitowiecki K. Prevalence of acanthocephalans in fishes of South Shetland Islands (Antarctic) III Metacanthocephalus johnstoni Zdzitowiecki, 1983, M. dalmori Zdzitowiecki, 1983 and notes on other species; general conclusions. Acta Parasitologica Polonica 1986; 31: 125-41.

67. Zdzitowiecki K. Little known and new Antarctic digenea species of the genera Neolepidapedon and Lepidapedon (Lepocreadiidae). Acta Parasitologica Polonica 1990; 35: 19-30.

68. Zdzitowiecki K. Reexamination of five Antarctic and subantarctic digenean and acanthocephalan species from Professor Szidat's collection. Acta Parasitologica Polonica 1990; 35: 31-6.

69. Zdzitowiecki K. Occurrence of Acanthocephalans in fishes of the open sea off the South Shetland Islands and South Georgia (Antarctica). Acta Parasitologica Polonica 1990; 35: 131-41.

70. Zdzitowiecki K. Occurrence of digeneans in open sea fishes off the South Shetland Islands and South Georgia, and the list of fish digeneans in the Antarctic. Pol Polar Res 1991; 12: 55-72.

71. Zdzitowiecki K. Antarctic representatives of the genus Lecithaster Lühe, 1901 (Digenea, Hemiuridae), with the description of a new species. Acta Parasitologica 1992; 37: 57-63.

72. Zdzitowiecki K. A contribution to the morphology of the Antarctic fish lepocreadiid digeneans, with a description of a new genus. Acta Parasitologica 1993; 38: 109-12.

73. Zdzitowiecki K. A description of Lecithochirium whitei sp.n. (Digenea, Hemiuroidea) from the Antarctic mesopelagic fish, Batihylagus antarcticus Günther. Acta Parasitologica 1994; 39: 1-4.

74. Zdzitowiecki K. Acanthocephala in fish in the Weddell Sea (Antarctica). Acta Parasitologica 1996; 41: 199-203.

75. Zdzitowiecki K. Digenea of fishes of the Weddell Sea. IV. Three Opecoelid species of the genera Neolebouria, Helicometra, and Stenakron. Acta Parasitologica 1997; 42: 138-43. 
76. Zdzitowiecki K. Digenea of fishes of the Weddell Sea V. Two new species of the genus Steringophorus (Fellodistomidae). Acta Parasitologica 1997; 42: 144-8.

77. Zdzitowiecki K. Digeneans of the families Opecoelidae and Lepocreadiidae, parasites of Lepidonotothen macrophthalma from the North Scotia Ridge, and remarks on the discrimination of Neolepidapedon magnatestis and N. trematomi. Acta Parasitologica 1999; 44: 233-40.

78. Zdzitowiecki K. Occurrence of endoparasitic worms in fish, Parachaenichthys charcoti (Bathydraconidae), off the South Shetland Islands (Antarctica). Acta Parasitologica. 2001; 46(1):18-23.

79. Zdzitowiecki K. New data on the occuurence of fish endoparasitic worms off Adelie Land, Antarctica. Pol Polar Res 2001; 22: 159-65.

80. Zdzitowiecki K. Occurrence of digenea in fishes of the family Channichthyidae in the Weddell Sea and other sub-continental areas of the Antarctica. Acta Parasitologica 2002; 47: 159-62.

81. Zdzitowiecki K. Occurrence of digenea in fishes of the family Nototheniidae in the Weddell Sea. Acta Parasitologica 2002; 47: 154-8.

82. Zdzitowiecki K. Occurrence of digenea in fishes of the family Artedidraconidae in the Weddell Sea and other areas of Antarctica. Acta Parasitologica 2002; 47: 306-9.

83. Zdzitowiecki K. Occurrence of digenea in fishes of the family Bathydraconidae in the Weddell Sea and other areas of Antarctica. Acta Parasitologica 2002; 47: 310-3.

84. Zdzitowiecki K. Occurrence of digenea in fishes other than Notothenioidei in the Weddell Sea and the whole Antarctica. Acta Parasitologica 2003; 48: 195-9.

85. Zdzitowiecki K, Rokosz B. Prevalence of acanthocephalans in fishes of South Shetland Islands (Antarctic). II. Aspersentis austrinus Van Cleave, 1929 and remarks on the validity of Heteracanthocephalus hureaui Dollfus, 1965. Acta Parasitologica Polonica 1986; 30: 161-71.

86. Zdzitowiecki K, Cielecka D. Digenea of fishes of the Weddell Sea. I. Parasites of Macrourus whitsoni (Gadiformes, Macrouridae). Acta Parasitologica 1997; 42: 23-30.

87. Zdzitowiecki K, Cielecka D. Digenea of fishes of the Weddell Sea. II. The Genus Macvicaria (Opecoelidae). Acta Parasitologica 1997; 42: 77-83.
88. Zdzitowiecki K, Cielecka D. Digenea of fishes of the Weddell Sea. III. The Lepocreadiidae (Genera Neolepidapedon and Lepidapedon), parasites of Notothenioidea. Acta Parasitologica 1997; 42: 84-91.

89. Zdzitowiecki K, Cielecka D. Digenea of the fish, Macrourus holotrachys (Gadiformes, Macrouridae), from the North Scotia Ridge, sub-Antarctic. Acta Parasitologica 1998; 43: 200-8.

90. Zdzitowiecki K, White MG. Acanthocephalan infection of insore fishes at the South Orkney Islands. Antarctic Sci 1996; 8: 273-6. [CrossRef]

91. Zdzitowiecki K, Zadrozny T. Endoparasitic worms of Harpagifer antarcticus off the South Shetland Islands (Antarctic). Acta Parasitologica 1999; 44: 125-30

92. Zdzitowiecki K, Laskowski Z. Helminths of an Antarctic fish, Notothenia coriiceps, from the Vernadsky Station (Western Antarctica) in comparison with Admiralty Bay (South Shetland Islands). Helminthologia 2004; 41: 201-7.

93. Zdzitowiecki K, Rocka A, Pisano E, Ozouf-Costaz C. A list of fish parasitic worms collected off Adelie Land (Antarctica). Acta Parasitologica 1998; 43: 71-4.

94. Zdzitowiecki K, Pisano E, Vacchi M. Additional data to Antarctic representatives of the genus Macvicaria Gibson et Bray, 71982 (Digenea, Opecoelidae), with a key to species occurring in the Antarctic. Acta Parasitologica 1992; 37: 131-4.

95. Zdzitowiecki K, Pisano E, Vacchi M. New data on the morphology and occurrence of a parasite of Antarctic fishes, Derogenes johnstoni Prudhoe et Bray, 1973 (Digenea, Hemiuridea). Acta Parasitologica 1993; 38: 69-71.

96. Zdzitowiecki K, Pisano E, Vacchi M. Antarctic representatives of the genus Neolebouria Gibson, 1976 (Digenea, Opecoelidae), with description of one new species. Acta Parasitologica. 1993; 38(1):11 14.97. Zdzitowiecki K, White MG, Rocka A. Digenean, monogenean and cestode infection of inshore fish at the South Orkney Islands. Acta Parasitologica 1997; 42 : 18-22.

97. Zdzitowiecki K, Palladino S, Vacchi M. Acanthocephala found in fish in the Terra Nova Bay (Ross Sea, Antarctica). Pol Polar Res 1999; 20: 56-63. 